\title{
Untersuchung der Wechselwirkung von Magnetfeldkonzentrationen und konvektiven Strömungen mit dem Strahlungsfeld in der Photosphäre der Sonne
}

\author{
Peter Vollmöller
}




\title{
Untersuchung der Wechselwirkung von Magnetfeldkonzentrationen und konvektiven Strömungen mit dem Strahlungsfeld in der Photosphäre der Sonne
}

\author{
Dissertation \\ zur Erlangung des Doktorgrades \\ der Mathematisch-Naturwissenschaftlichen Fakultäten \\ der Georg-August-Universität zu Göttingen
}

vorgelegt von

Peter Vollmöller

aus Fulda

Göttingen 2001 
D 7

Referent:

Prof. F. Kneer

Korreferent:

Prof. M. Schüssler

Tag der mündlichen Prüfung: 08.02.2002 
“... Eines zu sein mit allem, das ist Leben der Gottheit, das ist der Himmel des Menschen.

Eines zu sein mit allem, was lebt, in seliger Selbstvergessenheit wiederzukehren ins All der Natur, das ist der Gipfel der Gedanken und Freuden, das ist die heilige Bergeshöhe, der Ort der ewigen Ruhe, wo der Mittag seine Schwüle und der Donner seine Stimme verliert und das kochende Meer der Woge des Kornfelds gleicht.

Eines zu sein mit allem was lebt! Mit diesem Worte legt die Tugend den zürnenden Harnisch, der Geist des Menschen den Zepter weg, und alle Gedanken schwinden vor dem Bilde der ewigeinigen Welt, wie die Regeln des ringenden Künstlers vor seiner Urania, und das eherne Schicksal entsagt der Herrschaft, und aus dem Bunde der Wesen schwindet der Tod, und Unzertrennlichkeit und ewige Jugend beseligt, verschönert die Welt.

Auf dieser Höhe steh ich oft, mein Bellarmin! Aber ein Moment des Besinnens wirft mich herab. Ich denke nach und finde mich, wie ich zuvor war, allein, mit allen Schmerzen der Sterblichkeit, und meines Herzens Asyl, die ewig einige Welt, ist hin; die Natur verschließt die Arme, und ich stehe wie ein Fremdling vor ihr, und verstehe sie nicht.

Ach! wär ich nie in eure Schule gegangen. Die Wissenschaft, der ich in den Schacht hinunter folgte, von der ich, jugendlich töricht, die Bestätigung meiner reinen Freude erwartete, die hat mir alles verdorben.

Ich bin bei euch so recht vernünftig geworden, habe gründlich mich unterscheiden gelernt von dem, was mich umgibt, bin nun vereinzelt in der schönen Welt, bin so ausgeworfen aus dem Garten der Natur, wo ich wuchs und blühte, und vertrockne an der Mittagsonne.

Oh ein Gott ist der Mensch, wenn er träumt, ein Bettler, wenn er nachdenkt ... “

Hölderlin, Hyperion oder der Eremit in Griechenland 


\section{Inhaltsverzeichnis}

$\begin{array}{ll}\text { Zusammenfassung } & 10\end{array}$

1 Einführung, Motivation und Überblick 11

2 Gleichungssystem und Nebenbedingungen 17

2.1 Magnetohydrodynamische (MHD) Näherung . . . . . . . . . . 17

2.2 Maxwellsche Gleichungen . . . . . . . . . . . . . . . 17

2.3 Ohmsches Gesetz . . . . . . . . . . . . . . . . . 18

2.4 Induktionsgleichung . . . . . . . . . . . . . . . . 19

2.5 Magnetohydrodynamische Gleichungen . . . . . . . . . . . . 20

2.6 Ergänzungen zur Zustandsgleichung . . . . . . . . . . . 23

2.7 Strahlungsquellterm .................. 25

2.8 Funktionsform des resultierenden Gleichungssystem . . . . . . . 27

2.9 Rand- und Anfangsbedingungen . . . . . . . . . . . . . 28

2.9.1 Randbedingungen . . . . . . . . . . . . 29

2.9.2 Seitliche Ränder . . . . . . . . . . . . . . . . 29

2.9 .3 Oberer Rand . . . . . . . . . . . . . . . . . 29

2.9 .4 Unterer Rand . . . . . . . . . . . . . . 31 
2.9.5 Anfangsbedingungen und Ablauf der Simulation . . . . . . 36

3 Numerische Behandlung 39

3.1 Finite-Volumen-Verfahren auf Dreiecksgittern . . . . . . . . . 40

3.2 Godunov-Methode - Riemannlöser . . . . . . . . . . . . . 41

3.3 Konservative-Primitive Variablen . . . . . . . . . . . . 43

3.4 Dai-Wodward-Löser . . . . . . . . . . . . . . 47

3.5 HLL-Löser . . . . . . . . . . . . . . . . . . 48

3.6 Riemannlöser für partiell ionisierte Gase . . . . . . . . . . . . . 51

3.7 Gitteradaption . . . . . . . . . . . . . . 53

3.8 Zeitschrittweitensteuerung .................. 54

3.9 Entdimensionierung des Gleichungssystems . . . . . . . . . . . 54

4 Strahlungstransport $\quad 55$

4.1 Strahlungsstransport(RT)-Physikalische Annahmen . . . . . . . 55

4.2 RT-Löser . . . . . . . . . . . . . . . . . . . . . 57

4.2.1 Extended-Short-Characteristics (ESC)-Methode . . . . . 58

4.2.2 Realisation auf unstrukturierten Gittern . . . . . . . . . 60

4.2 .3 Oszillationskorrektur . . . . . . . . . . . 64

4.2.4 Periodische Randbedingungen . . . . . . . . . . . 64

4.3 Numerische Resultate . . . . . . . . . . . . . . . . . 66

4.3.1 Testproblem mit einer vorgegebenen exakten Lösung . . . . 67

4.3.2 Searchlight-Problem .............. . 74

4.3.3 Solare magnetische Flußschicht: Ein realitätsnaher Testfall . 75 
4.4 Numerische Bestimmung des Strahlungsquellterms _ . . . . . . . 81

4.4.1 Hydrodynamische Elementdaten . . . . . . . . . . . 82

4.4.2 Ordnung des Strahlungstransportlösers _ . . . . . . . . 86

4.4.3 Ausblick bei der numerischen $Q_{\mathrm{R}}$-Bestimmung . . . . . . 92

5 Magnetfeldverstärkung durch Konvektion 95

5.1 Hintergrund, bisherige Rechnungen $\ldots \ldots \ldots$. . . . . . 95

5.2 Simulationsresultate . . . . . . . . . . . . . . 96

$5.2 .1 \quad$ Diskussion . . . . . . . . . . . . . . . . . . 119

$\begin{array}{llr}6 & \text { Eigenschwingungen } & 121\end{array}$

6.1 Globale Schwingungsmuster der Sonne . . . . . . . . . . . . 121

6.2 Schwingungen kleinskaliger Magnetfeldkonzentrationen . . . . . 129

6.3 Schwingungsanalyse numerischer Simulationen . . . . . . . . 133

6.3.1 Fourier- oder Poweranalyse der Resultate des ST-Code . . . 137

6.3.2 Analyse der Resultate des WDV-Code . . . . . . . . . . . 144

6.3 .3 Diskussion $\ldots \ldots \ldots \ldots \ldots \ldots \ldots \ldots \ldots$

7 Ausblick 153

8 Anhang 155

8.1 Impliziter-Runge-Kutta-Löser $\ldots \ldots \ldots \ldots \ldots$

$\begin{array}{ll}\text { Danksagung } & 167\end{array}$

$\begin{array}{ll}\text { Lebenslauf } & 169\end{array}$ 


\section{Zusammenfassung}

In dieser Arbeit wurden die Entstehung von kleinskaligen Magnetfeldkonzentrationen, ihre Wechselwirkung mit photosphärischen Strömungsmustern und ihr Schwingungsverhalten mittels zweidimensionaler Simulationen der solaren Magnetokonvektion studiert.

- Es wurde ein numerisches Verfahren zur Lösung der Strahlungstransportgleichung entwickelt, das unabhängig von der Gittergeometrie auf 2- und 3-dimensionalen Rechengittern arbeiten kann. Dieses Verfahren ergibt bei vergleichbarer Rechenzeit einen um bis zu zwei Größenordungen kleineren numerischen Fehler als bisherige Löser, was sich insbesondere in den optisch dünnen Regionen stark auswirkt. Zusätzlich dazu können die starken Opazitätsvariationen im Rechengebiet, welche von der starken Temperaturabhängigkeit der Opazität herrühren, problemlos verarbeitet werden.

- Mit einem neuentwickelten 2D-MHD-Code (WDV-Code), der mit einem adaptiven Gitterkonzept, einem offenen unteren Rand und dem neuen Strahlungstransportlöser arbeitet, wurde die Ausbildung kleinskaliger Magnetfeldkonzentrationen als Folge der Wechselwirkung zwischen magnetischem Fluß und konvektiven Strömungen in einem $12000 \mathrm{~km}$ breiten und $1200 \mathrm{~km}$ tiefen photosphärischen Auschnitt simuliert. Dazu ist ein anfangs homogenes, vertikales Magnetfeld mit einer Feldstärke von 100 Gauß in die vollentwickelte hydrodynamische Konvektion eingebracht worden. Horizontale Strömungen transportieren den magnetischen Fluß in die konvektiven Abstromgebiete, wodurch Magnetfeldkonzentrationen entstehen. Die fortdauernde radiative Kühlung bewirkt innerhalb der Magnetfeldkonzentrationen verstärkte Abströmungen, die zu einer Evakuierung führen. Dadurch kommt es zu einer Verstärkung des Magnetfeldes auf Werte im Bereich von 1-2 kG.

- Die dynamische Wechselwirkungen von Magnetfeldkonzentrationen mit konvektiven Strömungen ist in Form einer Schwingungsanalyse untersucht worden. Es wurden eine mit dem WDV-Code und zwei mit dem ST-Code [Steiner et al. (1994) ([91])] durchgeführte Simulationen analysiert. Der ST-Code arbeitet ohne Gitteradaption, mit geschlossenem unteren Rand und einem herkömmlichen Strahlungstransportlöser. Ausserhalb der Magnetfeldkonzentrationen zeigen die Resultate beider Codes starke Beiträge, die durch akustische Eigenschwingungen $(4 \mathrm{mHz})$ und die Cutoff-Frequenz der Atmosphäre (5-6 mHz) bewirkte werden. Die WDV-Resultate zeigen zusätzlich noch deutliche von der Konvektion $(0-2 \mathrm{mHz})$ und akustischen Eigenschwingungen ( 3 und $7-8 \mathrm{mHz}$ ) verursachte Beiträge. Innerhalb der magnetischen Gebiete ergeben sich in beiden Fällen von akustischen Eigenschwingungen (4 mHz) und der Cutoff-Frequenz der longitudinalen Flußröhrenwellen (5$6 \mathrm{mHz}$ ) bewirkte Beiträge. Im Falle des WDV-Codes zeigen sich große Beiträge bei 0-2 $\mathrm{mHz}$ und 3-4 mHz, die von der Konvektion und den akustischen Eigenschwin-

gungen bewirkt werden. Im Gegensatz zum ST-Code zeigt der WDV-Code keine Beiträge bei $8-10 \mathrm{mHz}$. 


\section{Kapitel 1}

\section{Einführung, Motivation und Überblick}

Die sichtbare Oberfläche der Sonne zeigt eine wabenartige Strukturierung, welche als Granulation bezeichnet wird. Ihre Ursache sind die aus der unter der Photosphäre liegenden Konvektionszone aufsteigenden heißen Gasblasen. Sie besitzen typische Durchmesser von etwa $1000 \mathrm{~km}$ und sind durch kühle Gebiete, in denen die Materie wieder nach unten sinkt, voneinander getrennt. Die typische Zeitskala, in der ein einzelnes Granulum von seinem Auftauchen bis zu seinem Verschwinden beobachtbar ist, beträgt zwischen 5-10 min. Das Verschwinden der einzelnen Granulen kann auf zwei unterschiedliche Arten vonstatten gehen. Auf der einen Seite fragmentieren große Granulen, d.h. sie dehnen sich aus und zerfallen in zwei oder mehrere kleine Granulen. Auf der anderen Seite verringern kleinere Granulen unter dem Druck ihrer Nachbarn ihre Größe und lösen sich schließlich ganz auf ([65], [97]). Dieses instationäre Granuluationsmuster kennzeichnet die ruhigen, d.h. die unmagnetischen Gebiete der Sonne. In anderen Gebieten wird diese granulare Struktur lokal durch Magnetfeldansammlungen gestört. Die auffälligsten und größten sind die Sonnenflecken mit charakteristischen Durchmessern von einigen zehntausend Kilometern. Sie bestehen aus einem dunklen Kern, der Umbra, mit einer Magnetfeldstärke von ca. $3 \mathrm{kG}$, die von der nicht ganz so dunklen, stark zerfaserten Penumbra umgeben ist (Abb. 1.1). Am anderen Ende des beobachtbaren Größenspektrums stehen kleinskalige Magnetfeldkonzentrationen, im Folgenden auch Flußröhren genannt, welche bei Beobachtungen im kontinuierlichen Licht oder in Spektrallinien oft als kleinräumige signifikante Aufhellungen erkennbar sind (Abb. 1.1) und sich vorwiegend in den konvektiven Abstromgebieten befinden. Da in der solaren Photospäre und Konvektionszone die elektrische Leitfähigkeit des Plasmas sehr groß ist, gilt für die magnetische Reynoldszahl $R_{m} \gg 1$ $\left(R_{m}=U L / \eta ; \eta\right.$ : magnetische Diffusivtät, $U$ : typische Geschwindigkeit, L: typische Längenskala). In diesem Fall wird von eingefrorenen Feldlinien gesprochen, d.h. die magnetischen Feldlinien folgen den zu ihnen senkrechten Bewegungen des Plasmas und die horizontalen Strömungen konzentrieren den magnetischen Fluß in konvektiven Abstromgebieten, so daß die kleinskaligen Magnetfeldkonzentrationen 


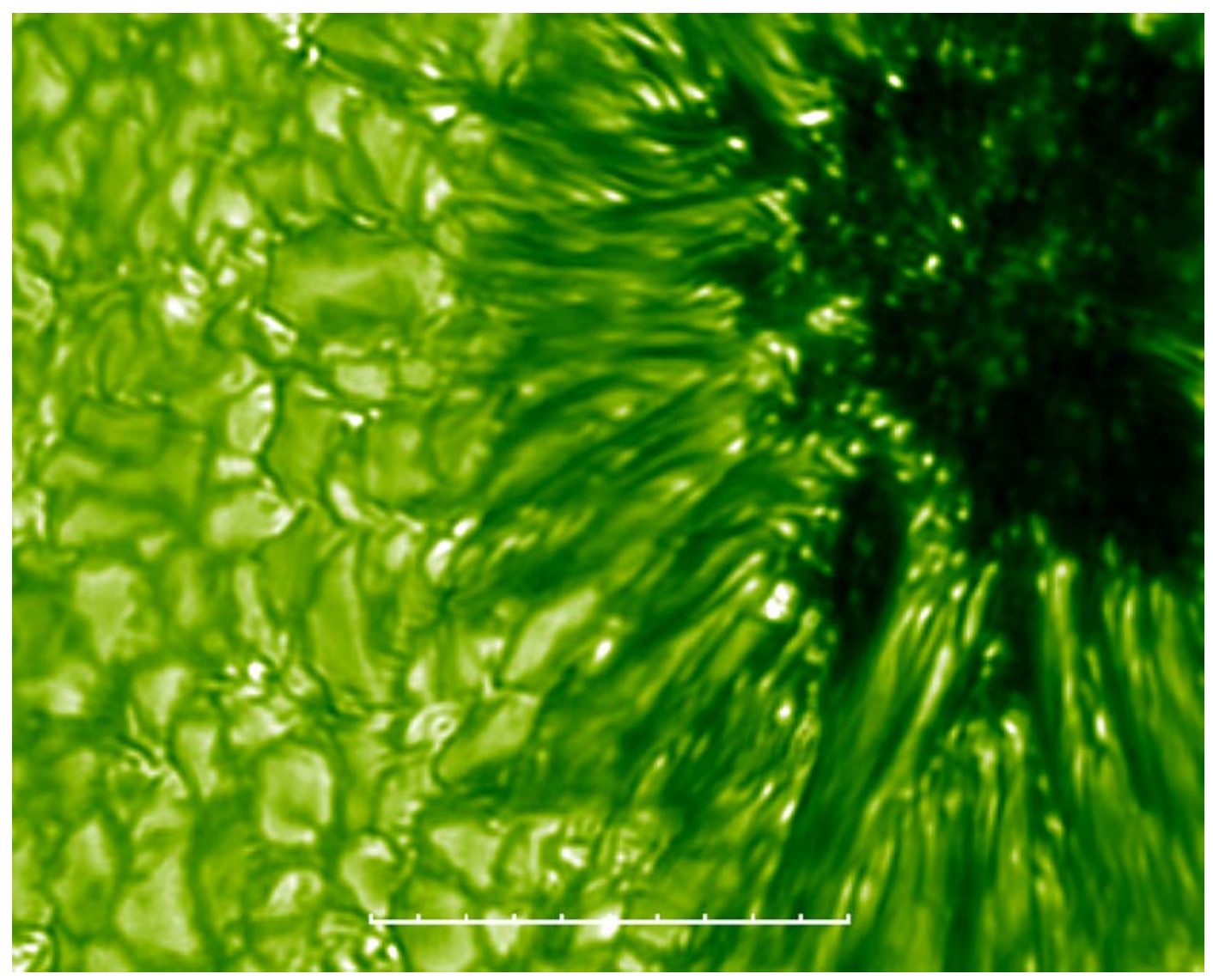

Abbildung 1.1: Ausschnitt eines Sonnenflecks mit einem dunklen Kern, der Umbra und der filamentierten Penumbra. Die Beobachtung ist im Wellenlängenbereich von 5500 Ådurchgeführt worden. Kleinräumige Magnetfeldkonzentrationen sind als signifikante Aufhellungen sichtbar und tauchen vorwiegend in intergranularen Gebieten auf. Die Gesamtlänge der weiß dargestellten Skala beträgt 10 Bogensekunden $(\approx 7500 \mathrm{~km}$ ) [Aufnahme F. Kneer, Sonnenobservatorium Teneriffa]

als das Resultat der dynamischen Wechselwirkung eines Magnetfelds mit den konvektiven Strömungsmustern verstanden werden müssen.

Die beobachteten typischen Durchmesser solcher Flußröhren betragen einige 100 km, was an der Grenze des räumlichen Auflösungsvermögen heutiger Teleskope liegt. Ihnen wird eine wichtige Bedeutung bei der koronalen Heizung beigemessen. Photosphärische Strömungen könnten die Flußröhren einerseits so stark schütteln, daß magnetohydrodynamische Wellen in der oberen Konvektionszone und Photosphäre erzeugt werden, die sich in die obere Sonnenatmosphäre ausbreiten und dort ihre Energie dissipieren. Andererseits könnten sich durch die Fußpunktbewegungen Magnetfeldkonzentrationen gegenseitiger Polarität in den oberen atmosphärischen Gebieten annähern, Stromschichten bilden und unter Dissipation magnetischer Energie rekonnektieren und so diese Bereiche heizen ([60], [63], [62]). Zusätzlich zu den lokal erzeugten magnetohydrodynamischen Wellen besitzt die Sonne eine Vielzahl von globalen akustischen Eigenschwingungen, die ein großes Spektrum von räumlichen Wellenzahlen überdecken. Sie existieren sowohl in der ruhigen Photosphäre und Chromosphäre als auch in Sonnenflecken und Flußröhren. 
Sie wurden im Fall der ruhigen Sonne von Leighton et al. entdeckt ([51]) und sind von Giovanelli et al. (1978), Solanki et al. (1992) und Volkmer et al. (1995) auch in magnetischen Flußkonzentrationen beobachtet worden. Sie besitzen eine Periode von etwa $300 \mathrm{~s}$ und werden mit den akustischen Eigenschwingungen identifiziert. Da Sonnenflecke sehr groß und dadurch leichter beobachtbar sind, ist ihre thermodynamische Struktur besser verstanden als die der kleinräumigen Flußkonzentrationen und etwaige Schwingungsmuster lassen sich besser auflösen und zuordnen. Um Aufschlüsse über die Schwingungsmuster kleinräumiger Flußkonzentrationen zu erzielen, sind Zeitserien räumlich und zeitlich hochaufgelöster Beobachtungen notwendig, die sehr schwierig unter den Bedingungen bodengebundener Messungen zu erlangen sind. Da direkte Beobachtungen von kleinräumigen magnetischen Strukturen durch das Auflösungsvermögen des optischen Instruments, insbesondere aber die Brechungsindexfluktuationen der Erdatmosphäre begrenzt sind ([77], [92]), lassen sich Erkenntnisse über ihre thermische Struktur und ihre innere Dynamik bislang überwiegend durch Einsatz indirekter Diagnosetechniken wie z.B. durch Spektralbeobachtungen polarisierter Strahlung gewinnen ([92], [80]).

Da die Dynamik kleinskaliger Magnetfeldkonzentrationen nur wenig verstanden ist und aus den skizzierten Gründen nur wenige Beobachtungen existieren, wählen wir in Form numerischer Simulationen einen theoretischen Weg. Dadurch erhoffen wir Aufschlüsse über die grundlegenden physikalischen Prozesse, die zur Ausbildung von Magnetfeldkonzentrationen führen und über charakteristische Schwingungsmuster und deren zugehörende Anregungsprozesse zu gewinnen.

Grundsätzlich beinhalten Simulationen der Wechselwirkung von Magnetfeldern und konvektiven Strömungen die numerische Lösung der magnetohydrodynamischen (MHD) Grundgleichungen, welche sich für ein ideales Plasma, als welches das photosphärische Plasma für die betrachteten Längen- und Zeitskalen angesehen werden kann, aus den Maxwellgleichungen und den hydrodynamischen Gleichungen unter Berücksichtigung der magnetohydrodynamischen Näherung und der Vernachlässigung der magnetischen Diffusion und der Viskosität ergeben ([67]).

In der Photosphäre der Sonne und in den obersten Schichten der Konvektionszone löst der Strahlungstransport die Konvektion als Energietransportmechanismus ab. Er beeinflußt somit die thermische Struktur der Flußröhren und ihrer Umgebung. Aus diesem Grunde muß er explizit berücksichtigt werden und findet in Form eines Quellterms in der Energiegleichung Eingang in das betrachtete Gleichungssystem. Da das betrachtete Plasma aus einem Gemisch von Elektronen und Ionen verschiedener Atomsorten mit jeweils verschiedener Ladungsstufe besteht und sich diese tiefenabhängige partielle Ionisation des Gases über das mittlere Molekulargewicht $\bar{\mu}(\rho, T)$ und die innere Energie auf die thermodynamischen Variablen $p, T$ und $\rho$ auswirkt, nimmt die Gaszustandsgleichung, die das System der MHD-Gleichungen schließt, eine kompliziertere Form an und die Dichte der totalen inneren Energie besteht fortan aus einem thermischen Anteil und einem durch die Ionisation bedingten. Seit Mitte der siebziger Jahre wurden mittels numerischer Simulationen die dynamischen Wechselwirkungen von Magnetfeldern und konvektiven Strömungen studiert. Die Augenmerke lagen dabei zum einen auf der Ausbildung kleinräumiger Magnetfeldkonzentrationen und ihrem Wechselspiel mit den granularen Strömungsmustern und zum anderen auf der thermischen und dynamischen Strukturierung bereits existierender Magnetfeldkonzentrationen und ihrem Energieaustausch mit ihrer Um- 
gebung.

Nordlund \& Stein (1990) ([59]) führten dazu dreidimensionale Simulationen der solaren Magnetokonvektion für einen solaren Auschnitt von $6 \times 6 \times 2.5 \mathrm{Mm}^{3}$ mit einer räumlichen Auflösung von $50 \mathrm{~km}$ durch. Dabei fanden sie, daß ein vertikales Magnetfeld mit einer mittleren Feldstärke von $0.5 \mathrm{kG}$, die granularen Strömungsmuster hin zu tiefen Schichten aufrecht erhält, so daß ein Netzwerk aus intergranularen Abstromgebieten auch $1000 \mathrm{~km}$ unter der sichbaren Sonnenoberfläche noch existent war und nicht in einzelne isolierte Abstromgebiete zerfiel wie im nichtmagnetischen Fall.

Grossmann-Doerth et al. (1998) ([36]) untersuchten mittels zweidimensionaler MHDSimulationen eines 2.4 Mm horizontal und 1.3 Mm vertikal ausgedehnten Ausschnitts der Photosphäre und der obersten Schichten der Konvektionszone bei einer horizontalen Gitterweite von $10 \mathrm{~km}$, unter Berücksichtigung der partiellen Wasserstoffionisation und des Strahlungstransport, die durch die horizontalen Strömungen bewirkte Konzentration magnetischen Flusses in den intergranularen Abstromgebieten und den in der zeitlichen Abfolge sich daran anschließenden konvektiven Kollaps ([104], [85], [61], [69]). Die damit verbundenen lokalen Ausbildungen starker Magnetfeldkonzentrationen sind das Resultat des Zusammenspiels von magnetfeldbedingter Unterdrückung der horizontalen Strömung, Strahlungskühlung der oberflächennahen Schichten und der superadiabatisch geschichteten Atmosphäre, welches zur Evakuierung der oberen Bereiche der Magnetfeldkonzentrationen und damit zu einer Feldverstärkung führt.

Steiner et al. (1998) ([90]) studierten mittels 2D-MHD-Simulationen die zeitabhängige Wechselwirkung einer magnetischen Flußschicht und ihrer Umgebung mit einer numerischen Gitterauflösung von $10 \mathrm{~km}$. Es zeigte sich, daß die externen Strömungen die Flußschicht zu transversalen Bewegungen mit großen horizontalen Geschwindigkeiten antreiben kann. Des Weiteren erzeugte die umgebende Granulation Druckfluktuationen, die sich in Form von vertikal propagierenden Wellen bis in die oberen atmosphärischen Bereiche ausbreiten und dort zu Schocks aufsteilen. Ploner et al. (2000) ([64]) führten für ein Rechengebiet von $6 \mathrm{Mm} \times 1.4 \mathrm{Mm}$ unter Berücksichtigung des Strahlungstransports und der Ionisation 2D-Simulationen der dynamischen Wechselwirkungen von bipolaren Magnetfeldkonzentrationen und konvektiven Strömungen mit einer maximalen numerischen Gitterauflösung von 25 $\mathrm{km}$ durch. Sie fanden, daß das recycling von magnetischem Fluß möglicherweise die beobachteten hohen Eruptionsraten von magnetischem Fluß in der ruhigen Sonne erklären kann.

Da der Einfluß der Randbedingungen auf die numerische Lösung möglichst gering gehalten werden soll, muß das Rechengebiet erheblich größer als die charakteristischen Strömungsstrukturen sein. Andererseits müssen die interessanten charakteristischen Strukturen wie z.B. Schockfronten genau aufgelöst werden und die Berechnungen in realistischer Zeit durchführbar sein. Dies legt sowohl die Verwendung eines adaptiven Gitterkonzepts mit lokaler Gitterverfeinerung als auch die Nutzung spezieller Rechnerarchitekturen wie Parallelrechner nahe.

Um ein großes Rechengebiet betrachten zu können und somit die dynamische Wechselwirkung mehrerer sich ausbildender Magnetfeldkonzentrationen mit der konvektiven Strömung untersuchen zu können, wird in dieser Arbeit die horizontale Ausdehnung des Rechengebietes auf $12000 \mathrm{~km}$ im Vergleich zu 6000 km wie bei Plo- 
ner et al. (2000) ([64]) oder $2400 \mathrm{~km}$ wie bei Steiner et al. (1998) ([90]) vergrößert. Dabei liegt aufgrund des adaptiven Gitterkonzeptes die maximale Gitterauflösung trotzdem bei knapp unter $10 \mathrm{~km}$. Zur Lösung des Gleichungssytems wird ein MHDRiemannlöser verwendet. Dieser Lösertyp ist durch eine sehr geringe numerische Diffusivität gekennzeichnet und wurde insbesondere zur numerischen Behandlung von Schockfronten entwickelt ([34]). Die Lösung des Gleichungssystems wird auf unstrukturierten Dreiecksgittern durchgeführt, da sich in diesem Kontext die lokale Gitterverfeinerung elegant umsetzen läßt ([46]).

Da das Strahlungstransportproblem ein nichtlokales Problem ist, ist die Auswahl der numerischen Lösungsverfahren im Hinblick auf die Verwendung von Rechnerarchitekturen mit verteiltem Speicher sehr wichtig. Die Berechnung des Strahlungsquellterms erfordert die Lösung der Strahlungstransportgleichung entlang einzelner Strahlen in definierten Raumrichtungen. Die direkte Lösung der Strahlungstransportgleichung entlang, durch das gesamte Rechengebiet 'laufender' Strahlen, wie sie von Mihalas et al. (1978) ([57]) vorgeschlagen wurde, birgt beim Einsatz von Parallelrechneren mit verteilten Speichern das Problem, daß zur Lösung der Strahlungstransportgleichung an einem Gitterpunkt die Informationen aller Gebietselemente, durch die der Strahl verläuft, notwendig sind. Dadurch wird das Schema aufgrund der erforderlichen Prozessorkommunikationen sehr ineffizient, obwohl die mit diesem Verfahren auf einem von dem MHD-Löser vorgegebenen Rechengitter erzielbare Genauigkeit in der Bestimmung der numerischen Lösung den Anforderungen entspricht. Die von Kunasz \& Auer (1988) ([47]) vorgeschlagene shortcharacteristics-Methode, welche von Bruls et al. (1999) ([4]) auf unstrukturierte Dreiecksgitter übertragen wurde, ist auf einem vorgegebenen Rechengitter bei der Berechnung der numerischen Lösung zu ungenau ([14]). Aus diesen Gegebenheiten leitet sich die Motivation zu dem ersten Teil der Arbeit ab, der in der Neuentwicklung eines numerischen Schemas zur Berechnung des Strahlungsquellterms bestand ([14]), welches in den Simulationen zum Einsatz kommt.

Im zweiten Teil der Arbeit soll zum einen die Ausbildung kleinskaliger Magnetfeldkonzentrationen in der solaren Photosphäre und oberen Konvektionszone als Folge der Wechselwirkung zwischen magnetischem Fluß und konvektiven Strömungen unter dem Einfluß des Strahlungsfeld untersucht werden. Zum anderen soll die dynamische Wechselwirkung von Magnetfeldkonzentrationen mit den konvektiven Strömungen im Hinblick auf eine mögliche Ausbreitung von Wellen in die oberen Bereiche der Sonnenphotosphäre in Form einer Schwingungsanalyse untersucht werden. Dazu wurden in ein 2D-MHD-Programm zur Lösung der magnetohydrodynamischen Grundgleichungen, das von den Herren A. Dedner und M. Wesenberg am Institut für Mathematik der Universität Freiburg (IAM) entwickelt wurde, der neuentwickelte Strahlungstransportlöser eingebaut und extensiv getestet. Desweiteren ist aufgrund der berücksichtigten partiellen Ionisation die Zustandsgleichung modifiziert und in tabellierter Form eingebaut worden. Mit dem veränderten und angepaßten 2D-MHD-Programm sind anschließend Simulationsrechnungen für solare Magnetokonvektion durchgeführt und analysiert worden. 


\section{Kapitel 2}

\section{Gleichungssystem und Nebenbedingungen}

\subsection{Magnetohydrodynamische (MHD) Näherung}

Es sollen die Wechselwirkungen von Magnetfeldern mit Strömungen in einem gut leitenden, stoßdominierten, quasineutralen Plasma mit $v \ll c$ beschrieben werden $(v$ : Materiegeschwindigkeit, $c$ : Lichtgeschwindigkeit). Unter diesen Voraussetzungen verbindet sich die Hydrodynamik mit der Elektrodynamik zur Magnetohydrodynamik [Kippenhahn\& Möllenhoff (1973), [42]].

\subsection{Maxwellsche Gleichungen}

$$
\begin{aligned}
\nabla \cdot \vec{B} & =0 \\
\nabla \times \vec{B} & =\frac{4 \pi}{c} \vec{j}+\frac{1}{c} \partial_{t} \vec{E} \\
\nabla \cdot \vec{E} & =4 \pi \tilde{\rho} \\
\nabla \times \vec{E} & =\frac{1}{c} \partial_{t} \vec{B}
\end{aligned}
$$

Hierbei sind $\vec{j}$ und $\tilde{\rho}$ die Strom- bzw. Ladungsdichte. In einem kontinuierlichen Medium schreibt man die Maxwellschen Gleichungen üblicherweise mit dem Magnetfeld $\vec{H}$ und der dielektrischen Verschiebung $\vec{D}$, welche im obigen Gleichungssystem durch die magnetische Induktion $\vec{B}$ und das elektrische Feld $\vec{E}$ ersetzt wur- 
den, wobei gilt

$$
\vec{D}=\epsilon \vec{E} \quad, \quad \vec{H}=\frac{\vec{B}}{\mu}
$$

( $\epsilon$ : Dielektrizitätskonstante, $\mu$ : magnetische Permeabilität).

Da im betrachteten Bereich der Sonne die Strömungsgeschwindigkeiten sehr viel kleiner als die Lichtgeschwindigkeit sind, kann in der nichtrelativistischen Näherung gerechnet werden. Wie man unten sieht, kann aus diesem Grunde der zum Verschiebungsstrom proportionale Term $1 / c \partial_{t} \vec{E}$ in Gl. (2.2) vernachlässigt werden. Unter der Annahme, daß $l_{0}$ und $t_{0}$ typische Längen- und Zeitskalen sind, lassen sich die Terme in Gl. (2.1) - (2.4) abschätzen. Dazu ersetzen wir die Differentialoperatoren durch Quotienten der Skalengrössen. Mit den typischen Werten $E_{0}$ und $B_{0}$ für $|\vec{E}|$ und $|\vec{B}|$ läßt sich Gl. (2.4) nun größenordnungsmäßig abschätzen zu

$$
|\nabla \cdot \vec{E}| \approx \frac{E_{0}}{l_{0}} \approx \frac{B_{0}}{c t_{0}} \approx\left|\frac{\partial \vec{B}}{\partial t}\right| .
$$

Mit Hilfe dieser Beziehung kann der Verschiebungsstrom in Gl. (2.2) approximiert werden:

$$
\left|\frac{1}{c} \frac{\partial \vec{E}}{\partial t}\right| \approx \frac{E_{0}}{c t_{0}} \approx \frac{B_{0}}{l_{0}} \frac{l_{0}^{2}}{c^{2} t_{0}^{2}} \approx \frac{v_{0}^{2}}{c^{2}}|\nabla \times \vec{B}| .
$$

Es werden nur Terme mit $\mathcal{O}\left(\frac{v_{0}}{c}\right)$ berücksichtigt, weil $v_{0} \equiv l_{0} / t_{0} \ll c$ gilt und der Verschiebestrom $\mathcal{O}\left(\frac{v_{0}^{2}}{c^{2}}\right)$ ist, fällt er weg und Gl. (2.2) ergibt sich zu:

$$
\nabla \times \vec{B}=\frac{4 \pi}{c} \vec{j}
$$

\subsection{Ohmsches Gesetz}

Im Ruhesystem des Plasmas hat das Ohmsche Gesetz die folgende Form:

$$
\vec{j}=\sigma \vec{E}
$$

wobei $\sigma$ die elektrische Leitfähigkeit ist. In einem mit der Geschwindigkeit $|\vec{v}| \ll c$ relativ zum ruhenden Bezugssystem bewegten Plasma transformiert sich das elektrische Feld wie $\vec{E} \longrightarrow \vec{E}+\frac{1}{c}(\vec{v} \times \vec{B})$ und das Ohmsche Gesetz bekommt die Form:

$$
\vec{j}=\sigma\left[\vec{E}+\frac{1}{c}(\vec{v} \times \vec{B})\right] .
$$




\subsection{Induktionsgleichung}

Unter Zuhilfenahme des Ohmschen Gesetzes Gl. (2.10) lassen sich die Gleichungen (2.8) und (2.4) umformulieren, so daß daraus die Induktionsgleichung für die Leitfähigkeit $\sigma=$ const. folgt:

$$
\partial_{t} \vec{B}=\underbrace{\nabla \times(\vec{v} \times \vec{B})}_{\text {Induktion }}+\underbrace{\frac{c^{2}}{4 \pi \sigma} \nabla^{2} \vec{B}}_{\text {Diffusion }} .
$$

Diese beschreibt die zeitliche Entwicklung des Magnetfelds $\vec{B}$ bei gegebenem Strömungsfeld $\vec{v}$ und besteht aus einem Induktions- und einem Diffusionsterm. Der Induktionterm beschreibt dabei die Veränderung des Magnetfeldes, die durch das Strömungsfeld hervorgerufen wird. Der Diffusionsterm hingegen beschreibt den Zerfall des Magnetfeldes durch Ohmsche Dissipation. Das Verhältnis von Induktionsund Diffusionsterm entspricht der magnetischen Reynoldszahl $R_{m}$ :

$$
R_{m}:=\left|\frac{\nabla \times(\vec{v} \times \vec{B})}{\eta \nabla \times(\nabla \times)}\right| \approx \frac{l_{0} v_{0}}{\eta}
$$

$(\eta:=1 /(\mu \sigma):$ magnetische Diffusivität).

Für $R_{m} \ll 1$ ist der Induktionsterm in Gl. (2.11) vernachlässigbar, weshalb diese die Form einer Diffusionsgleichung bekommt und den Ohmschen Zerfall des Magnetfeldes beschreibt. Die typische Zeitskale des Zerfalls hängt dabei von der räumlichen Ausdehnung des betrachteten Systems ab.

$$
\tau_{\text {diff }}:=\frac{l_{0}^{2}}{\eta}
$$

Für $R_{m} \gg 1$ ist der Ohmsche Zerfall des Magnetfeldes vernachlässigbar. In diesem Fall wird von ‘eingefrorenen Feldlinien' gesprochen. Die Bewegung des Plasmas verformt das Feld in einer Weise, als ob es gegenüber Bewegungen senkrecht zur Feldrichtung in das Plasma eingefroren wäre. Die Änderung des Magnetfeldes wird in diesem Grenzfall einzig durch die Plasmabewegung bestimmt. In der solaren Konvektionszone ist der Grenzfall $R_{m} \gg 1$ sehr gut erfüllt. Um $R_{m}$ für die in der oberen solaren Konvektionzone herrschenden Verhältnisse abschätzen zu können, wird der von Spitzer (1962) ([82]) angegebene Ausdruck für die elektrische Leitfähigkeit eines vollständig ionisierten Gases verwendet. Nach Einsetzen aller Größen ergibt sich folgende Temperaturabhängigkeit der Leitfähigkeit in der Konvektionszone (Stix 1989, S.253, [92]):

$$
\sigma \approx 0.003 T^{3 / 2} \frac{A}{V m} \approx 10^{4} \frac{A}{V m}
$$


Bei einem typischen Flußröhrendurchmesser von $200 \mathrm{~km}$ in der oberen Konvektionszone, einer Bewegungszeitskala, welche durch die hydrodynamische Zeitskala von $t_{0} \approx 600 \mathrm{~s}$ bestimmt ist und der magnetischen Permeabilität $\mu_{0}=4 \pi \cdot 10^{-7}$ folgt eine typische Geschwindigkeit $v_{0} \approx 333 \mathrm{~m} / \mathrm{s}$ und damit eine magnetische Reynoldszahl in der oberen Konvektionszone von:

$$
R_{m} \approx 8 \cdot 10^{5}
$$

Obwohl in der solaren Photosphäre und der unteren Chromosphäre die Temperatur so niedrig ist, daß Wasserstoff und Helium fast vollständig neutral sind, werden von schweren Elementen mit niedrigem Ionisationspotential noch genügend Elektronen geliefert, um einen Ladungsfluß aufrecht zu erhalten, der einem $\sigma \approx 10^{2} \frac{A}{V m}$ entspricht (Stix 1989, S.254, [92]). Unter der Annahme eines typischen Flußröhrendurchmessers in diesen atmosphärischen Bereichen von $d \approx 600 \mathrm{~km}$ und der Verwendung der hydrodynamische Zeitskala von $t_{0} \approx 600 \mathrm{~s}$ folgt eine magnetische Reynoldszahl von $R_{m} \approx 7 \cdot 10^{5}$ in den Bereichen der unteren Photosphäre. Somit ist auch in diesen Regionen die magnetische Diffusion vernachlässigbar und das Bild der 'eigefrorenen Feldlinien' angemessen. Im Weiteren gehen wir von einem Plasma unendlicher Leitfähigkeit aus $(\sigma=\infty)$, so daß der zweite Term auf der rechten Seite in Gl. (2.11) verschwindet und sie folgende Form annimmt:

$$
\partial_{t} \vec{B}=\nabla \times(\vec{v} \times \vec{B})=\nabla(\vec{v} \vec{B}-\vec{B} \vec{v})=0
$$

\subsection{Magnetohydrodynamische Gleichungen}

Sowohl die Magnetohydrodynamik wie die Plasmaphysik beschäftigen sich mit der Untersuchung leitender Flüssigkeiten oder Gase unter Einwirkung elektromagnetischer Felder. Das Phänomen der Leitung wird bekanntlich durch freie oder quasifreie Elektronen verursacht, die sich unter der Wirkung äußerer Felder bewegen können. Somit werden die Elektronen von äußeren Feldern beschleunigt, erfahren durch Stöße allerdings eine Richtungsänderung, so daß ihrer Bewegung in Richtung des Feldes eine effektive Reibungskraft entgegensteht, welche proportional der Stoßfrequenz ist. Die Trennungslinie zwischen Magnetohydrodynamik und Plasmaphysik ist nicht sehr scharf. Doch existieren separate Bereiche, auf die sich die Vorstellungen eines der beiden Gebiete anwenden lassen. Ist die Frequenz der angelegten Felder sehr viel größer als die Stoßfrequenz, dann werden die Elektronen und Ionen durch elektrische Felder zwischen aufeinanderfolgenden Stößen in entgegengesetzte Richtungen beschleunigt und sie versuchen sich räumlich zu trennen. Durch die Ladungstrennung werden starke elektrostatische Rückstellkräfte induziert und es kommt zu oszillatorischen Schwankungen in der Ladungsdichte, den sogenannten Plasmaschwingungen.

In leitenden Flüssigkeiten oder dichten, ionisierten Gasen ist die Stoßfrequenz selbst bei hoher Leitfähigkeit noch so groß, daß es zwischen den Elektronen und Ionen abgesehen von einer Art Zitterbewegung bei hohen Frequenzen zu keiner Ladungs- 
trennung kommt. Die auftretenden elektrischen Felder rühren entweder von der Bewegung der Flüssigkeit her, die einen Stromfluß verursacht, oder aber von zeitlich veränderlichen magnetischen Feldern oder Ladungsverteilungen außerhalb der Flüssigkeit. Die mechanische Bewegung des Systems läßt sich somit als Bewegung einer einkomponentigen Flüssigkeit betrachten und ist durch die Evolutionsgleichungen der Hydrodynamik, den Navier-Stokes-Gleichungen, beschreibbar. Dieses System von Gleichungen setzt sich aus den nachfolgend erläuterten Gleichungen zusammen.

- Kontinuitätsgleichung

$$
\partial_{t} \rho+\nabla \cdot(\rho \vec{v}) \equiv \frac{D \rho}{D T}+\rho(\nabla \cdot \vec{v})=0 \operatorname{mit} \frac{D}{D T} \equiv \frac{\partial}{\partial t}+\vec{v} \cdot \nabla
$$

Die Kontinuitätsgleichung beschreibt die Massenerhaltung. So führt beispielsweise die Einströmung in ein definiertes Gebiet am Orte $\vec{r}(\nabla \cdot(\rho \vec{v})<0) \mathrm{zu}$ einer Dichtezunahme $(\partial \rho / \partial t>0)(\rho$ : Massendichte, $\vec{v}$ : Materiegeschwindigkeit). Die substantielle Ableitung $\frac{D}{D T}$ beschreibt die Änderung einer physikalischen Größe entlang ihres Weges durch das Plasma.

- Impulsgleichung

$$
\partial_{t}(\rho \vec{v})+\nabla \cdot\left(\rho \vec{v} \vec{v}^{T}+\mathcal{P}\right)=\vec{F}_{e}+\vec{F}_{\nu}
$$

mit

$$
\begin{aligned}
\mathcal{P} & =\left(p+\frac{1}{c}(\vec{J} \times \vec{B})\right) \\
& =\left(\left(p+\frac{B^{2}}{8 \pi}\right) \delta_{i j}-\frac{B_{i} B_{j}}{4 \pi}\right)_{i, j=1}^{3} .
\end{aligned}
$$

$\left(\vec{g}\right.$ : Schwerebeschleunigung, $p$ : Gasdruck und $\frac{B^{2}}{8 \pi}$ : magnetischer Druck, $B_{i}, B_{j}$ Magnetfeldkomponenten (mit $\left.i, j=1,2,3\right)$ ). Die magnetische Kraft oder Lorenz-Kraft $(\vec{J} \times \vec{B})$ im Spannungstensor $\mathcal{P}$ setzt sich aus dem magnetischen Druck $B^{2} / 8 \pi$ und einer zusätzlichen Kraft, welche sich als Spannung entlang von magnetischen Kraftlinien interpretieren läßt, zusammen. Die Impulsgleichung beschreibt die Änderung der Impulsdichte, welche durch den am Ort $\vec{r}$ des Elements herrschenden Druckgradient $\nabla \cdot p$, die äußeren Kräfte $\vec{F}_{e}$ und die viskose Kraft $\vec{F}_{\nu}$ bedingt ist. Mit der kinematischen Viskosität $\nu$ läßt sich $\vec{F}_{\nu}$ für ein kompressibles Gas in der folgenden allgemeinen Form schreiben ([40]):

$$
\vec{F}_{\nu}=\rho \nu\left(\nabla^{2} \vec{v}+\frac{1}{3} \nabla(\nabla \cdot \vec{v})\right)
$$


Das Verhältnis der Größenordnung der Trägheitskraft zur viskosen Kraft in Gleichung Gl. (2.18) definiert die Reynoldszahl Re:

$$
\left|\frac{\rho D \vec{v} / D t}{\vec{F}_{\nu}}\right| \approx \frac{l_{0} v_{0}}{\nu} \equiv R_{e} .
$$

Dabei sind $v_{0}$ und $l_{0}$ typische Längen bzw. Geschwindigkeiten (Kap. 2.4). Für ein vollständig ionisiertes Wasserstoff-Plasma gibt Spitzer ([82]) folgende Temperaturabhängigkeit von $\nu$ an:

$$
\rho \nu=2.21 \cdot 10^{-16} \frac{T^{5 / 2}}{\ln \Lambda} \frac{\mathrm{kg}}{\mathrm{ms}}
$$

$\ln \Lambda$ stellt dabei den Coulomb-Logarithmus mit $5 \leq \ln \Lambda \leq 10$ für den betrachteten solaren Bereich dar. Mit den typischen Werten für den Flußröhrendurchmesser $d \approx 200 \mathrm{~km}-600 \mathrm{~km}$ und die hydrodynamische Zeitskala $t_{0} \approx 600$ s folgt:

$$
R e \approx 10^{8}
$$

Somit ist die viskose Kraft vernachlässigbar und das System der NavierStokes Gleichungen reduziert sich auf das System der Eulergleichungen ([49]). Im hier betrachteten Fall wirkt nur die Gravitationskraftdichte $\vec{F}_{e}=\rho \vec{g}$ mit $\vec{g}=-g \mathbf{e}_{\mathbf{z}}$ und $g=|\vec{g}|=$ const als äußere Kraft auf das System.

- Energiegleichung

Die zeitliche Entwicklung der Gesamtenergiedichte, welche sich aus den Dichten der kinetischen, der potentiellen, der inneren und der magnetischen Energie am Orte $\vec{r}$ zusammensetzt:

$$
E=e_{k i n}+e_{p o t}+e_{i n n}+e_{m a g}=\frac{1}{2} \rho \vec{v}^{2}+\rho \vec{g}+\rho \epsilon+\frac{B^{2}}{8 \pi},
$$

wird beschrieben durch die Erhaltungsgleichung der Gesamtenergie:

$$
\partial_{t} E+\nabla \cdot(\rho E \vec{v}+\mathcal{P} \vec{v})=Q_{\mathrm{R}} .
$$

Die Dichte der totalen inneren Energie setzt sich dabei aus dem thermischen Anteil und dem Ionisationsanteil zusammen: $\epsilon=\epsilon_{\text {therm }}+\epsilon_{\text {ion }}$. Der Quellterm $Q_{\mathrm{R}}$ beschreibt die Energieflußdichte durch Strahlung und wird im folgenden noch näher erläutert.

- Zustandsgleichung

Die thermische Zustandsgleichung eines Gases stellt den Zusammenhang zwischen Massendichte $\rho$, Temperatur T, Gasdruck $p$ und dem relativen mittleren Molekulargewicht $\bar{\mu}(\rho, T)$ am Orte $\vec{r}$ her

$$
p=\frac{\rho R_{*} T}{\bar{\mu}},
$$

und schließt das Gleichungssystem

( $R_{*}=k / A_{0}$ : allgemeine Gaskonstante, $k$ : Boltzmann-Konstante, $A_{0}$ : atomare Masseneinheit). 


\subsection{Ergänzungen zur Zustandsgleichung}

Die solare Materie wird durch ein homogenes Gemisch idealen Gases beschrieben. Es setzt sich zu ca. $73 \%$ aus Wasserstoff, zu 25\% aus Helium und den verbleibenden $2 \%$ aus schwereren Elementen zusammen. Alle auftretenden Teilchen sind einatomig. Die relativen Anzahldichten werden durch die nachfolgend dargestellten dimensionslosen Zahlen $\nu_{l}$ repräsentiert:

$$
\nu_{H}=0.9081, \quad \nu_{H e}=0.0908, \quad \nu_{M e}=0.0011
$$

mit

$$
\nu_{H}+\nu_{H e}+\nu_{M e}=1
$$

Im Falle eines partiell ionisierten Gases wirkt sich die tiefenabhängige Ionisation des Gases bzw. der tiefenabhängige Ionisationsgrad $\xi$ des Gases über das mittlere Molekulargewicht $\bar{\mu}(\rho, T)$ auf die thermodynamischen Variablen $p, T$ und $\rho$ und somit die Gesamtenergie Gl. (2.25) aus.

Das mittlere Molekulargewicht hat im neutralen Fall die Form:

$$
\bar{\mu}_{0}=\frac{1}{A_{0}} \sum_{l} \nu_{l} A_{l}
$$

wobei $A_{0}=1.660 \cdot 10^{-24} \mathrm{~g}$ die atomare Masseneinheit ist und ergibt sich mit

$$
A_{H}=1.673 \cdot 10^{-24} \mathrm{~g} \quad A_{H e}=6.645 \cdot 10^{-24} \mathrm{~g}, \quad A_{M e}=28.22 \cdot 10^{-24} \mathrm{~g}
$$

$\mathrm{zu}$

$$
\bar{\mu}_{0}=1.297
$$

Hier wird vorerst nur die Wasserstoffionisation berücksichtigt werden. Dies ist als ein erster Schritt näherungsweise dadurch gerechtfertigt, daß die Heliumionisation bei $T \approx 13000 \mathrm{~K}$ in einem Modell der Konvektionszone weniger als $0.1 \%$ erreicht, die Wasserstoffionisation hingegen etwa 20\%. Die Ionisation der schweren Elemente wird vernächlässigt, da ihre Häufigkeit mit $2 \%$ sehr gering ist. Im Falle partieller Ionisation verändert sich die Form des mittleren Molekulargewicht zu:

$$
\bar{\mu}=\frac{n \bar{\mu}_{0}}{n+n_{e}}=\frac{\bar{\mu}_{0}}{1+\frac{n_{e}}{n}}
$$

( $n$ : Anzahldichte der Atome und Ionen, $n_{e}$ : Anzahldichte freier Elektronen).

$\mathrm{Zu}$ dessen Bestimmung verbleibt nun das Problem der Berechnung des Quotienten $\frac{n_{e}}{n}$. Die Anzahldichte $n$ der Atome und Ionen ist, für den unserem Rechengebiet 
entsprechenden atmosphärischen Bereich gegeben durch:

$$
n=n_{H}+n_{H+}+n_{H e}+\sum_{j} n_{j, S E l}+\sum_{j, i} n_{j, S E l}^{i} .
$$

Dabei stellt $\sum_{j} n_{j, S E l}$ die Anzahldichte schwerer Elemente und $\sum_{j, i} n_{j, S E l}^{i}$ die i-te Ionisationsstufe des j-ten schweren Elements dar. Grundsätzlich berechnet sich $n_{e}$ aus dem System gekoppelter Sahagleichungen:

$$
n_{e} \frac{n_{\nu+1}}{n_{\nu}}=\frac{u_{\nu+1}}{u_{\nu}} \frac{2\left(2 \pi m_{e} k T\right)^{3 / 2}}{h^{3}} \exp \left(-\chi_{\nu} / k T\right)
$$

$\left(n_{\nu+1}, n_{\nu}\right.$ : Anzahldichte der $\nu+1$-fach und der $\nu$-fach ionisierten Atome, $u_{\nu+1}, u_{\nu}$ : quantenmechanische Zustandssumme der Zustände $\nu+1$ und $\nu, \chi_{\nu}$ : Anregungsenergie, $m_{e}$ : Elektronenmasse, $h^{3}$ : Phasenraumvolumenelement, $k$ : BoltzmannKonstante).

Dabei entspricht die Anzahl notwendiger Gleichungen dem Produkt aus der Anzahl betrachteter Elemente und der Anzahl deren Ionisationsstufen. Im allgemeinen wird man dieses (nichtlineare) System iterativ lösen. Da wir nur die Wasserstoffionisation berücksichtigen, gibt es nur eine Sahagleichung, die sich für $D:=\frac{n_{H}^{+}}{n_{H}}$ folgendermaßen darstellt:

$$
D=\frac{\left(2 \pi m_{e}\right)^{3 / 2}(k T)^{5 / 2} e^{-\frac{\chi_{H}}{k T}}}{h^{3}\left(\frac{D}{1+D}\right) \frac{\rho}{\bar{\mu}_{0} A_{0}} \nu_{H} k T} .
$$

Dies führt zu einer quadratischen Gleichung für $D$. Unter Einbeziehung des Ionisationsgrades für $H$ :

$$
\xi_{H}=\frac{n_{H+}}{n_{H}+n_{H+}}
$$

und

$$
n_{e}=\xi_{H} \nu_{H} n
$$

ergibt sich die totale innere Energiedichte $\rho \epsilon \mathrm{zu}$ :

$$
\begin{array}{r}
\rho \epsilon=\frac{3}{2}\left(\frac{\rho}{\bar{\mu}_{0} A_{0}}+\left(\frac{D}{1+D}\right) \nu_{H} \frac{\rho}{\bar{\mu}_{0} A_{0}}\right)+ \\
\left(\frac{D}{1+D}\right) \frac{\rho \nu_{H}}{\bar{\mu}_{0} A_{0}} \chi_{H}
\end{array}
$$

$\left(\chi_{H}=13.598 \mathrm{eV}\right.$, Ionisierungsenergie des Wasserstoff). 


\subsection{Strahlungsquellterm}

Der radiative Energietransport ist in der solaren Photosphäre von entscheidender Bedeutung. In der Konvektionszone ist der dominierende Energietransportmechanismus die Konvektion, welche in der darüberliegenden Photosphäre durch den radiativen Energietransport abgelöst wird. Um die dynamische Wechselwirkung zwischen Magnetfeld und Konvektion mittels numerischer Simulationen untersuchen zu können, muß der Transport von Strahlung durch das Plasma berücksichtigt werden. Die Kopplung von Magnetohydrodynamik und Strahlungstransport führt dabei zu einem Quellterm in der Evolutionsgleichung der Gesamtenergiedichte. Die Einführung eines solchen Quellterms ist durch die Tatsache begründet, daß $v \ll c$ ( $v$ : typische hydrodynamische Geschwindigkeit, $c$ : Lichtgeschwindigkeit) gilt, die Lichtlaufzeit also sehr viel geringer ist als alle hydrodynamischen Zeitskalen und somit der Energieaustausch der Strahlung mit ihrer Umgebung als instantan betrachtet werden kann. Die Ermittelung dieses Strahlungsquellterms besteht dabei aus drei aufeinanderfolgenden Schritten:

- Die Berechnung der spezifischen Intensität $I_{\nu}(\vec{r}, \vec{\mu})$.

- Die Integration der Spezifischen Intensität über den diskretisierten Raumwinkel.

- Die Berechnung des Strahlungsquellterms $Q_{\mathrm{R}}$.

Allgemein läßt sich der Transport von elektromagnetischer Strahlung und deren Wechselwirkung mit Materie durch die zeit- und frequenzabhängige Stahlungstransportgleichung beschreiben (im folgenden als RT-Gleichung bezeichnet):

$$
\frac{1}{c} \partial_{t} I_{\nu}+\vec{\mu} \cdot \nabla I_{\nu}+\kappa_{\nu} \rho I_{\nu}=\kappa_{\nu} \rho S_{\nu}
$$

$I_{\nu}(\vec{r}, \vec{\mu})$ ist proportional der Strahlungsenergie $\mathrm{d} E_{\nu}^{r a d}(\vec{r}, \vec{\mu})$, welche von Strahlung der Frequenz $\nu, \nu+d \nu$ durch die Fläche $d f \cos \theta$ in den Raumwinkel $d \Omega$ transportiert wird

$$
I_{\nu}(\vec{r}, \vec{\mu}) d f \cos \theta d \Omega=d E_{\nu}^{r a d}(\vec{r}, \vec{\mu})
$$

Unter der Annahme $v \ll c$, reduziert sich Gl. (2.39) auf die stationäre Form $\left(\frac{1}{c} \partial_{t} I_{\nu}=\right.$ $0)$ :

$$
\vec{\mu} \cdot \nabla I_{\nu}=\kappa_{\nu} \rho\left(S_{\nu}-I_{\nu}\right)
$$




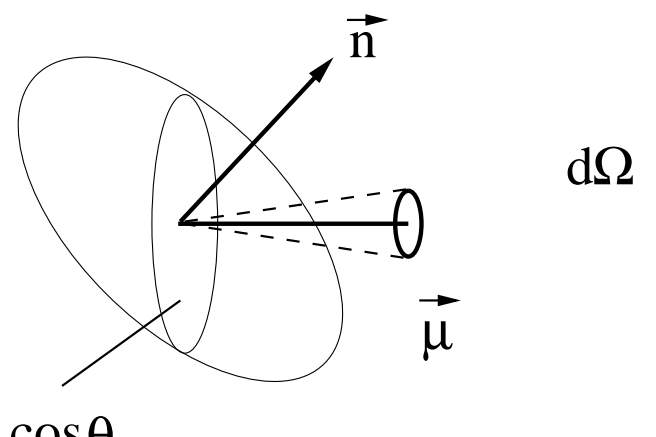

\section{df $\cos \theta$}

Abbildung 2.1: Die spezifische Intensität ist proportional der Strahlungsenergie $d E_{\nu}^{r a d}(\vec{r}, \vec{\mu})$, welche von Strahlung der Frequenz $\nu, \nu+d \nu$ durch die Fläche $d f \cos \theta$ in den Raumwinkel $d \Omega$ transportiert wird.

welche es zu lösen gilt. Zur Berechnung des Strahlungsquellterms $Q_{R}(\vec{r})$ gibt es zwei äquivalente Formulierungen ([56], [4]):

$$
\begin{aligned}
\mathrm{Q}_{\mathrm{R}}(\vec{r}) & =4 \pi \rho \int_{\nu}(\underbrace{\kappa_{\nu} J_{\nu}}_{\text {absorbierte Strahlung }}-\underbrace{\kappa_{\nu} S_{\nu}}_{\text {emittierte Strahlung }}) d \nu, \\
\text { und } & \\
\mathrm{Q}_{\mathrm{R}}(\vec{r}) & =\int_{\nu} \nabla \cdot \vec{F}_{\nu}^{r a d} d \nu .
\end{aligned}
$$

Die mittlere Intensität $J_{\nu}$ ist die am Orte $\vec{r}$ über alle Raumwinkel $\vec{\mu}$ integrierte spezifische Intensität und hat folgende Form:

$$
J_{\nu}(\vec{r})=\frac{1}{4 \pi} \int I_{\nu}(\vec{r}, \vec{\mu}) d \mu
$$

Der Strahlungsstrom $\vec{F}_{\nu}^{r a d}(\vec{r})$ hingegen ist das über alle Raumwinkel $\vec{\mu}$ integrierte Produkt des Winkels $\vec{\mu}$ und der spezifischen Intensität am Orte $\vec{r}$ :

$$
\vec{F}_{\nu}^{r a d}(\vec{r})=\int I_{\nu}(\vec{r}, \vec{\mu}) \vec{\mu} d \mu
$$

( $\vec{\mu}$ : Strahlungsrichtung, $\nu$ : Frequenz,$\kappa_{\nu}(\rho, T)$ : Absortionskoeffizient, $I_{\nu}(\vec{r}, \vec{\mu})$ : spezifische Intensität, $S_{\nu}$ : Quellfunktion, $\rho$ : Massendichte). 


\subsection{Funktionsform des resultierenden Gleichungssy- stem}

Im Falle von zweidimensionalen numerischen Untersuchungen setzt sich das zu lösende Gleichungssystem aus den Gleichungen der Magnetohydrodynamik für zwei räumliche Dimensionen ( $x, z)$ (Kap. 2.5) (für ein gravitativ geschichtetes Medium) und der Zustandsgleichung (Kap. 2.6) zusammen. Zur Bestimmung des Strahlungsquellterms $Q_{\mathrm{R}}$ Gl. (2.42) ist zusätzlich die stationäre RT-Gleichung für die spezifische Intensität Gl. (4.1) zu lösen.

In kartesischer Komponentendarstellung hat es folgende Form:

$$
\begin{gathered}
\partial_{t} \vec{U}+\partial_{x} \vec{F}(\vec{U})+\partial_{z} \vec{G}(\vec{U})=\vec{Q}(\vec{U}) \\
\nabla \cdot \vec{B}=0 \\
p=\frac{\rho R_{*} T}{\bar{\mu}}
\end{gathered}
$$

mit

$$
\begin{aligned}
\vec{U}:=\vec{U}(\vec{r}, t) & :=(\rho, \rho \vec{v}, \vec{B}, E)^{T} \\
& =\left(\rho, \rho v_{x}, \rho v_{y}, \rho v_{z}, B_{x}, B_{y}, B_{z}, E\right)^{T}
\end{aligned}
$$

und

$$
\vec{F}(\vec{U}):=\left(\begin{array}{c}
\rho v_{x} \\
\rho v_{x}^{2}+p+\frac{\vec{B}^{2}}{8 \pi}-\frac{B_{x}^{2}}{4 \pi} \\
0 \\
\rho v_{x} v_{z}-\frac{B_{z} B_{x}}{4 \pi} \\
0 \\
0 \\
v_{x} B_{z}-v_{z} B_{x} \\
\left.p+\frac{\vec{B}^{2}}{8 \pi}\right)-\frac{B_{x}}{4 \pi} \vec{B} \cdot \vec{v}
\end{array}\right)
$$




$$
\vec{Q}(\vec{U}):=\left(\begin{array}{c}
0 \\
0 \\
0 \\
-\rho g \\
0 \\
0 \\
0 \\
-\rho v_{z} g+Q_{\mathrm{R}}
\end{array}\right)
$$

\subsection{Rand- und Anfangsbedingungen}

Da es sich bei dem betrachteten Gleichungssystem Gln. (2.46) um ein System partieller, nichtlinearer Differentialgleichungen für den Variablenvektor $\vec{U}(\vec{r}, t)$ handelt, müssen zur Ermittelung einer eindeutigen Lösung Nebenbedingungen gestellt werden. So fordern die auftauchenden Ableitungen nach dem Ort Randbedingungen und die Ableitungen nach der Zeit Anfangsbedingungen. Die höchste Ableitung der Variablen nach dem Ort entspricht der Anzahl zu stellender Randbedingungen, während die höchste Ableitung nach der Zeit die Anzahl der zu stellenden Anfangsbedingungen vorgibt. Im konkreten Fall ist die zu simulierende solare Konvektion charakterisiert durch warme breite Aufstromgebiete und kalte konzentrierte Abstromgebiete. Letztere sind in der Photosphäre auf einer räumlichen Skala von etwa $1000 \mathrm{~km}$ mehr oder weniger intermittent verteilt und organisieren sich in tieferen Schichten zu einigen wenigen Abstromgebieten, die möglicherweise sogar den Boden der Konvektionzone erreichen können. Obwohl es von größtem Interesse wäre, können wir nicht die gesamte Konvektionszone, welche sich über $2 \cdot 10^{5} \mathrm{~km}$ vertikal erstreckt und die Photosphäre in einem Simulieren und gleichzeitig die numerische Auflösung so wählen, daß die kleinskalige dynamische Interaktion von Konvektion und Magnetfeld im Übergangsbereich zwischen Konvektionszone und Photosphäre entsprechend aufgelöst wird. Aus diesem Grunde beschränken wir uns in unserer Simulation auf einen Ausschnitt, der den oberen Teil der Konvektionzone und die gesamte Photosphäre umfaßt. Das Rechengebiet ist in vertikaler Richtung 1400 km und in horizontaler Richtung $12000 \mathrm{~km}$ ausgedehnt und stellt somit nur einen begrenzten Ausschnitt aus dem zu untersuchenden solaren Gebiet dar. Dies hat zur Konsequenz, daß sowohl Annahmen über die physikalischen Eigenschaften der angrenzenden Gebiete, als auch über jene zu Beginn der numerischen Simulation gemacht werden müssen. Da diese das magnetohydrodynamische Strömungsmuster im Simulationsgebiet maßgeblich beeinflussen, müssen sie möglichst realitätsnah gewählt werden. 


\subsubsection{Randbedingungen}

Unser Rechengebiet wird von 4 Rändern einbeschrieben, die in Abb. 2.2 als oberer (top), unterer (bot) und seitlicher Rand bezeichnet sind.

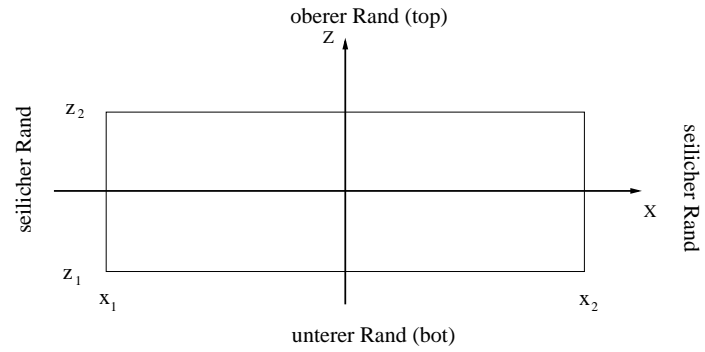

Abbildung 2.2: Schematische Darstellung des Rechengebiets mit den Randbezeichnungen.

\subsubsection{Seitliche Ränder}

An den beiden seitlichen Rändern, welche parallel zur $z$-Achse verlaufen, werden periodische Randbedingungen für den Variablenvektor $\vec{U}(x, z, t)$ vorgeschrieben, d.h. jede Variable ist periodisch in $x$-Richtung.

$$
\vec{U}\left(x_{1}, z, t\right)=\vec{U}\left(x_{2}, z, t\right) .
$$

\subsubsection{Oberer Rand}

Am oberen geschlossenen Rand darf keine Materie durch den Rand strömen. Dieser soll undurchlässig und perfekt leitend sein. Im Falle eines gravitativ geschichteten Mediums lassen sich die Bedingungen für den Variablenvektor $\vec{U}\left(x, z_{2}, t\right)$ am oberen Rand numerisch, im Finiten-Volumen- und Riemannlöserkontext mittels sogenannter Randelemente oder Geisterzellen umgesetzen. Dabei gehören die Randelemente oder Geisterzellen nicht mehr zum eigentlichen Rechengebiet (Abb.2.3). Im Folgenden sind die Bedingungen für den Variablenvektor erläutert, wobei $b c$ das Randelement und $d c$ die an das Randelement angrenzende Gitterzelle, welche noch zum eigentlichen Rechengebiet gehört, bezeichnen.

- Mit der Forderung nach einem geschlossenen Rand ergibt sich für die Massendichte, daß die Normalableitung der Massendichte verschwinden muß und sie somit über den Rand hinweg in die Geisterzelle symmetrisch fortgesetzt wird :

$$
\frac{\partial \rho^{t o p}}{\partial z}=0 \quad, \quad \text { für } \quad \rho^{b c}=\rho^{d c}
$$




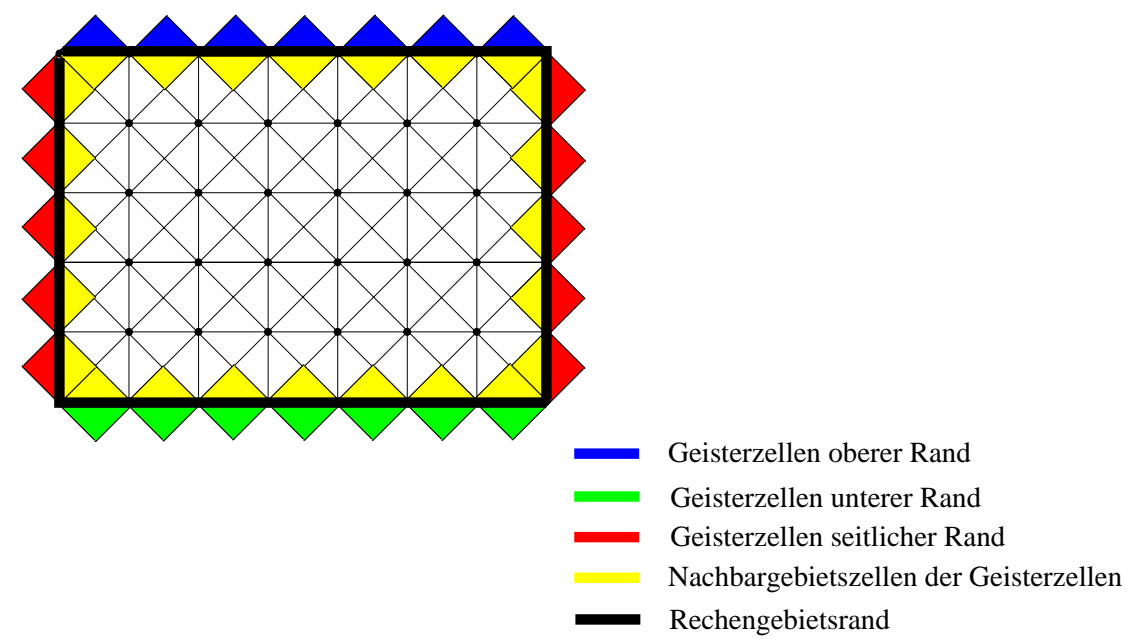

Abbildung 2.3: Schematische Darstellung des numerischen Rechengitters bestehend aus Dreieckszellen mit den farbig markierten Randelementen (Geisterzellen) (blau : oberer Rand, rot : seitlicher Rand, grün unterer Rand) und den Nachbargebietselementen (gelb).

- Die vertikale Geschwindigkeitskomponente $v_{z}$ verschwindet nur dann am Gebietsrand, wenn sie über den Rand hinweg antisymmetrisch fortgesetzt wird. Während die Horizontalkomponente $v_{x}$ symmetrisch fortgesetzt werden muß, damit ihre Normalableitung $v_{x}$ verschwindet:

$$
\begin{aligned}
v_{z}^{\text {top }}=0, & \text { für } \quad v_{z}^{b c}=-v_{z}^{d c}, \\
\frac{\partial v_{x}^{\text {top }}}{\partial z}=0, & \text { für } \quad v_{x}^{b c}=v_{x}^{d c} .
\end{aligned}
$$

- Mit den Annahmen, daß am Rand die Horizontalkomponente des Magnetfeld $B_{x}=0$ ist und der magnetische Fluß im Gesamtgebiet erhalten sein soll, folgt für $B_{x}$ und $B_{z}$ :

$$
\begin{aligned}
B_{x}^{\text {top }}=0, & \text { für } \quad B_{x}^{b c}=0 \quad, \\
\frac{\partial B_{z}^{\text {top }}}{\partial z}=0, & \text { für } \quad B_{z}^{b c}=B_{z}^{d c} .
\end{aligned}
$$

- Aus den Bedingungen für die Massendichte und das Geschwindigkeitsfeld folgt für die Gesamtenergiedichte, daß die Normalableitung der Gesamtenergiedichte verschwinden muß und die Gesamtenergiedichte somit über den Rand hinweg symmetrisch fortgesetzt werden muß:

$$
\frac{\partial E^{t o p}}{\partial z}=0, \quad \text { für } \quad E^{b c}=E^{d c} .
$$




\subsubsection{Unterer Rand}

Da zwischen der Wärmeerzeugung im Sonneninneren und der radiativen Abstrahlung derselben Wärmemenge an der Oberfläche $2 \cdot 10^{7}$ Jahre vergehen, kann der Wärmetransport von innen nach außen als stationärer Prozeß angesehen werden. Der konvektive Energiefluß und der radiative Strahlungsfluß unterscheiden sich insofern, als daß sie die transportierte Energiemenge pro Zeit- und pro Flächeneinheit zweier, in unterschiedlichen solaren Tiefen lokalisierten, Energietransportmechanismen beschreiben. In der Konvektionszone wird die Wärme vorwiegend durch Konvektion transportiert, in der Photosphäre hingegen durch Strahlung, die näherungweise bei $\tau_{5000}=1$ abgestrahlt wird. Im Weiteren gilt:

$$
F_{\odot}^{k o n}=F_{\odot}^{r a d}=6.6344 \cdot 10^{10}\left[\frac{e r g}{s^{1} \mathrm{~cm}^{2}}\right]
$$

$\left(F_{\odot}^{k o n}:\right.$ konvektiver Energiefluß, $F_{\odot}^{\text {rad }}$ : radiativer Strahlungsfluß, $\tau_{5000}$ : optische Tiefe bei $5000 \AA$ ).

Im Folgenden werden zwei mögliche Behandlungen des unteren Randes erläutert. Da der untere Rand unseres Rechengebietes in der oberen Konvektionszone liegt, wird dabei die in das Rechengebiet einströmende, konvektiv transportierte Energie auf unterschiedliche Arten berücksichtigt.

- Geschlossener unterer Rand: In diesem Fall ist der Rand für Strömungen undurchlässig und der Variablenvektor $\vec{U}\left(x, z_{1}\right)$ wird analog zum Fall des geschlossenen oberen Rand gewählt. Allerdings wird die Temperatur am unteren Rand zeitlich konstant gehalten und das Gebiet geheizt. Der konvektive Wärmeeintrag in das Gebiet wird durch einen Heizungsterm in der Energiegleichung beschrieben. Für diesen Heizungsquellterm $Q_{\text {Heiz }}$ gilt:

$$
\begin{aligned}
& Q_{\text {Heiz }}(z)= \begin{cases}0 & : \quad z>z_{h} \\
\frac{F_{\odot}^{\text {rad }}}{\int_{z_{1}}^{z_{1}} f(z) d z}: & z_{1}<z<z_{h}\end{cases} \\
& \text { mit } f(z)=\frac{z-z_{1}}{z_{h}-z_{1}} e^{-\left(z-z_{h}\right)}
\end{aligned}
$$

( $z_{1}$ : unterer Rechengebietsrand, $z_{h}$ : oberer Rand des geheizten Gebiets).

- Offener unterer Rand : Im folgenden schließen sich die Erläuterungen einer heuristischen offenen Randbedingung an. Heuristisch in dem Sinn, daß entsprechende Annahmen über die physikalischen Bedingungen am unteren Rand des Simulationsgebietes gemacht werden. Grundsätzlich unterscheiden wir zwischen räumlichen Bereichen, in denen über den unteren Rand in das Gebiet Einströmung stattfindet, und solche, in denen aus dem Gebiet über den Rand Materie ausströmt. Dabei sollte ein offener Rand konvektiven Strömungen und Wellen gestatten, ungestört in das Rechengebiet zu gelangen. Da die Konvektionszone in der Nähe des unteren Randes zu einem hohen Grad 
als adiabatisch geschichtet anzusehen ist, werden für ein konvektiv aufsteigendes idealisiertes Volumenpaket dort die Dichte der inneren Energie und die korrespondierende Temperatur $T$ als räumlich und zeitlich konstant und als bekannt betrachtet. Weiterhin führt das große Verhältnis zwischen der Schallgeschwindigkeit und den typischen hydrodynamischen Geschwindigkeiten (20 : 1) in der Nähe des unteren Randes dazu, daß jede Druckstörung instantan verschwindet, ohne die hydrodynamischen Variablen im Restgebiet zu beeinflussen. Für den Gasdruck $p^{b o t}(x, t)$ am unteren Rand ergibt sich daher:

$$
\begin{array}{r}
\partial_{t} p^{b o t}(x, t) \neq 0, \quad \partial_{x} p^{b o t}(x, t)=0 \\
\text { und } \quad p^{b c}(x, t)=p^{b o t}(x, t)
\end{array}
$$

$\longrightarrow$ Einströmung:

- In den Einströmungsgebieten wird die Temperatur am unteren Rand so gewählt, daß der zeitliche Mittelwert des berechneten radiativen Strahlungsflusses am oberen Rand dem solaren Strahlungsfluß entspricht :

$$
<F_{\text {sim }}>_{\tau_{K H}}=F_{\odot}^{r a d}
$$

mit

$$
T=T^{b o t}=\text { const. }
$$

und

$$
T^{b c}=T^{b o t}
$$

Dabei wurde der zeitliche Mittelwert über die thermische Zeitskala oder Kelvin-Helmholtz-Zeitskala $\tau_{K H}$, welche das Verhältnis von der gesamten im Rechengebiet vorhandenen thermischen Energiedichte und der pro Fläche, Sekunde und Massendichte abgestrahlten Energie darstellt, gebildet. $\left(\tau_{K H}\right.$ : Kelvin-Helmholtz-Zeitskala, $F_{\text {sim }}$ : berechneter Stahlungsfluß am oberen Rechengebietsrand).

- Unter Benutzung der Zustandsgleichung Gl. (2.27) läßt sich nun die Massendichte ermitteln :

$$
\rho_{\text {ein }}^{b o t}=\frac{p^{b o t} \bar{\mu}\left(T^{b o t}, p^{b o t}\right)}{T^{b o t} R_{g a s}}, \quad \text { und } \quad \rho^{b c}=\rho_{\text {ein }}^{b o t} .
$$

- Für den Impuls und das Geschwindigkeitsfeld am unteren Rand folgt unter Berc̈ksichtigung der Massenflußerhaltung:

$$
\frac{\partial(\rho \vec{v})_{e i n}^{b o t}}{\partial z}=0, \quad \text { für }(\rho \vec{v})^{b c}=(\rho \vec{v})^{d c} \longrightarrow \vec{v}^{b c}=\vec{v}^{d c} \rho^{b c}
$$

- Mit den Annahmen, daß am Rand die Horizontalkomponente des Magnetfeld $B_{x}=0$ ist und der magnetische Fluß im Gesamtgebiet erhalten 
sein soll, gilt für $B_{x}$ und $B_{z}$ :

$$
\begin{array}{cl}
B_{x}^{b o t}=0 \quad, & \text { für } \quad B_{x}^{b c}=0 \\
\frac{\partial B_{z}^{b o t}}{\partial z}=0 \quad, & \text { für } \quad B_{z}^{b c}=B_{z}^{d c} .
\end{array}
$$

- Die Gesamtenergiedichte in den Einströmungsgebieten ermittelt sich zu

$$
E_{\text {ein }}^{b o t}=\epsilon_{\text {inn }}^{b o t}\left(\rho^{b o t}, p^{b o t}\right)+e_{k i n}^{b o t}+e_{m a g}^{b o t}+e_{\text {source }}^{b o t}
$$

für

$$
E_{\text {ein }}^{b c}=\epsilon_{\text {inn }}^{b c}\left(\rho^{b c}, p^{b c}\right)+e_{k i n}^{b c}+e_{\text {mag }}^{b c}+e_{\text {source }}^{b c}
$$

Um sicherzustellen, daß Gl. (2.58) gilt, wurde in Gl. (2.65) ein Quellterm eingeführt, welcher folgende Form hat:

$$
e_{\text {source }}^{b c}=\left(\frac{F_{\odot}^{r a d}-F_{\odot}^{s i m}}{F_{\odot}^{r a d}}\right) e_{0} \cdot \frac{d t}{\tau_{K H}}
$$

und die über den Gebietsunterrand einströmende Menge an innerer Energie in jedem Zeitinkrement $d t$ entsprechend regelt $\left[e_{0}:=e_{0}\left(T^{b o t}, p^{b o t}(t)\right)\right]$.

$\longrightarrow$ Ausströmung :

- Eine adiabatische Ausströmung ist angenommen worden:

$$
\frac{\partial\left(\frac{p}{\rho^{\gamma}}\right)_{\text {aus }}^{\text {bot }}}{\partial z}=0 \longrightarrow \rho_{\text {aus }}^{\text {bot }}, \quad \text { und } \quad \rho^{b c}=\rho_{\text {aus }}^{\text {bot }} \text {. }
$$

- Für die Normalableitung des Impulses und das Geschwindigkeitsfeld gilt :

$$
\frac{\partial(\rho \vec{v})_{a u s}^{\text {bot }}}{\partial z}=0 \longrightarrow \vec{v}_{\text {aus }}^{b o t}, \quad \text { und } \quad \vec{v}^{b c}=\vec{v}_{a u s}^{b o t}
$$

- Für das Magnetfelds gilt :

$$
\begin{gathered}
\frac{\partial B_{z}^{b o t}}{\partial z}=0 \\
\text { und } \\
B_{x}^{b o t}=0
\end{gathered} .
$$

- Massendichte, Impulsdichte, Magnetfeld, Druck und Temperatur legen die Gesamtenergiedichte in den Ausströmungsgebieten entsprechend Gl. (2.25) fest. 
Da im gesamten Rechengebiet $|\vec{v}| \ll c_{s}$ gilt und somit die Abweichungen von der Hydrostatik gering sind, kann es aus physikalischer Sicht nicht zu starken Massefluktuationen kommen. Die Formulierung der unteren Randbedingung hingegen induziert starke, mehr oder weniger periodisch auftretende Fluktuationen in der Gesamtmasse, welche die Ausbildung kleinskaliger Strömungsstrukturen stark unterbinden würde. Um diese zu eliminieren wird in jedem Zeitinkrement $d t$ die Erhaltung der Gesamtmasse gefordert:

$$
\frac{d M}{d t}=0
$$

und somit das Verschwinden des totalen vertikalen Masseflusses über den Unterrand:

$$
\int_{x_{1}}^{x_{2}} \rho^{n}(x) v_{z}^{n}(x) d x=0
$$

(n : n-ter Zeitpunkt).

Dies unterbindet lokale Ein- und Ausströmungen über den Unterrand nicht. Die Summe der individuellen vertikalen Masseflüsse verschwindet allerdings.

Bewirkt wird dies durch eine horizontal homogene Justierung des Drucks $p^{b o t}(t)$ zu jeder Zeit $t$, so daß sich für die Dichte, den Impuls und die Energiedichte der ein- und ausströmende Bereiche Werte ergeben, welche das Verschwinden des totalen vertikalen Massefluß über den Unterrand bewirken. Nachfolgend ist der Justierungsablauf schematisch skizziert. 


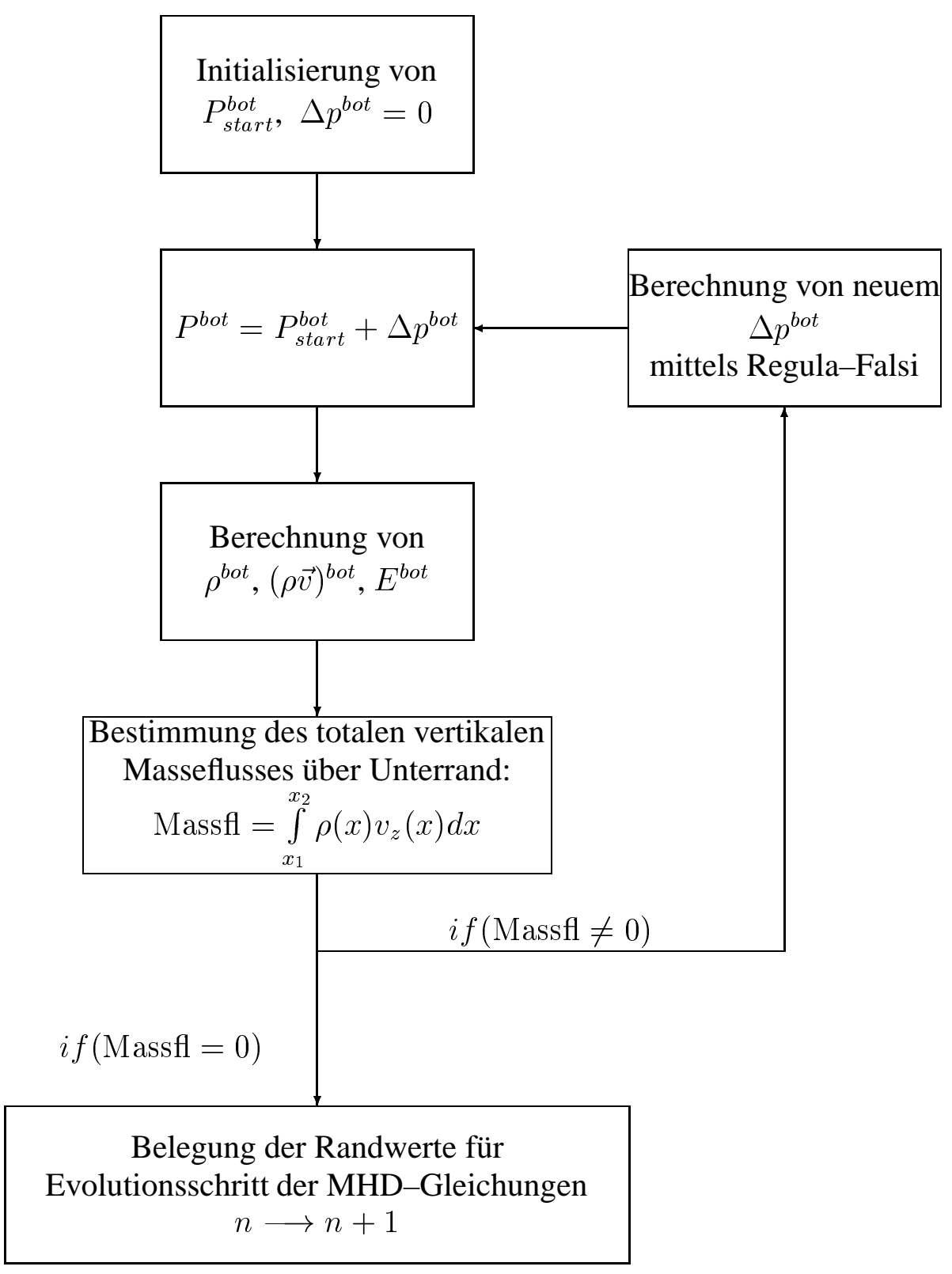




\subsubsection{Anfangsbedingungen und Ablauf der Simulation}

Die Lösungen der MHD-Gleichungen sind von der Zeit abhängig, was aus den auftretenden Zeitableitungen folgt. Wie bereits erwähnt, ergibt sich daraus die Notwendigkeit des Stellens von Zusatzbedingungen an die Lösung. Dies erfolgt in Form von Anfangsbedingungen, die den physikalischen Zustand des Systems beschreiben, von dem aus die zeitliche Entwicklung des Systems berechnet werden soll. Die Gesamtsimulation besteht aus zwei unterschiedlichen Simulationsphasen. Dabei wird die erste Phase als hydrodynamische bezeichnet. Zum Zeitpunkt $t=0$ werden dabei die Anfangsbedingungen für die thermodynamischen Variablen Temperatur $T$, Massendichte $\rho$ und Druck $p$ festgelegt. Mit diesen Bedingungen und den bereits erwähnten Randbedingungen für ein am oberen und unteren Rand abgeschlossenes und an den seitlichen Rändern periodisch fortgesetztes Rechengebiet (siehe Kap. 2.9.4) wird das Gleichungssystem Gl. (2.46) gelöst und die zeitliche Entwicklung des Anfangszustands berechnet. Die magnetohydrodynamische Simulationsphase beginnt, nachdem sich vollständige hydrodynamische Konvektion ausgebildet hat. Dazu wird ein homogenes vertikales Magnetfeld von $B_{0}=100$ Gauss angeschaltet. Die eigentliche magnetohydrodynamische Simulation wird nun mit dem, um das Magnetfeld $\vec{B}$ und die magnetische Energie $\frac{\vec{B}^{2}}{8 \pi}$, erweiterten Variablenvektor $\vec{U}^{h y d}\left(x, z, t_{h y d}\right)$ der hydrodynamischen Phase (hyd : kennzeichnet den Endzustand des Variablenvektors nach der hydrodynamischen Simulationsrechnung) und dem offenen unteren Rand gestartet.

- Startinitialisierung (hydrodynamische Simulationsphase) :

\section{- Thermodynamische Schichtung}

Als Anfangsbedingungen für die thermodynamischen Variablen $T, \rho$ und $p$ als Funktion der Höhe $z$, welche durch Gl. (2.27) untereinander verbunden sind, dient das eindimensionale Modell der ruhigen Photosphäre und Konvektionszone von Spruit ([83]) (Abb. 2.4).

- Geschwindigkeit

Das Geschwindigkeitsfeld wird in Form eines quellenfreien Doppelwirbels $(\vec{\nabla} \cdot \vec{v}=0)$ mit einer höhenunabhängigen Amplitude von $A_{m}=1$ $\mathrm{km} / \mathrm{s}$, vorgegeben:

$$
\begin{aligned}
& v_{x}(x, z)=A_{m} \sin \left(k_{x} x\right) \cos \left(k_{z} z\right), \\
& v_{z}(x, z)=A_{m} \cos \left(k_{x} x\right) \sin \left(k_{z} z\right) .
\end{aligned} .
$$

- Magnetfeld:

$$
\vec{B}=\left(0,0, B_{0}\right) \quad \text { mit } \quad B_{0}=0 \text { Gauss } .
$$

- Magnetohydrodynamische Simulationsphase : 

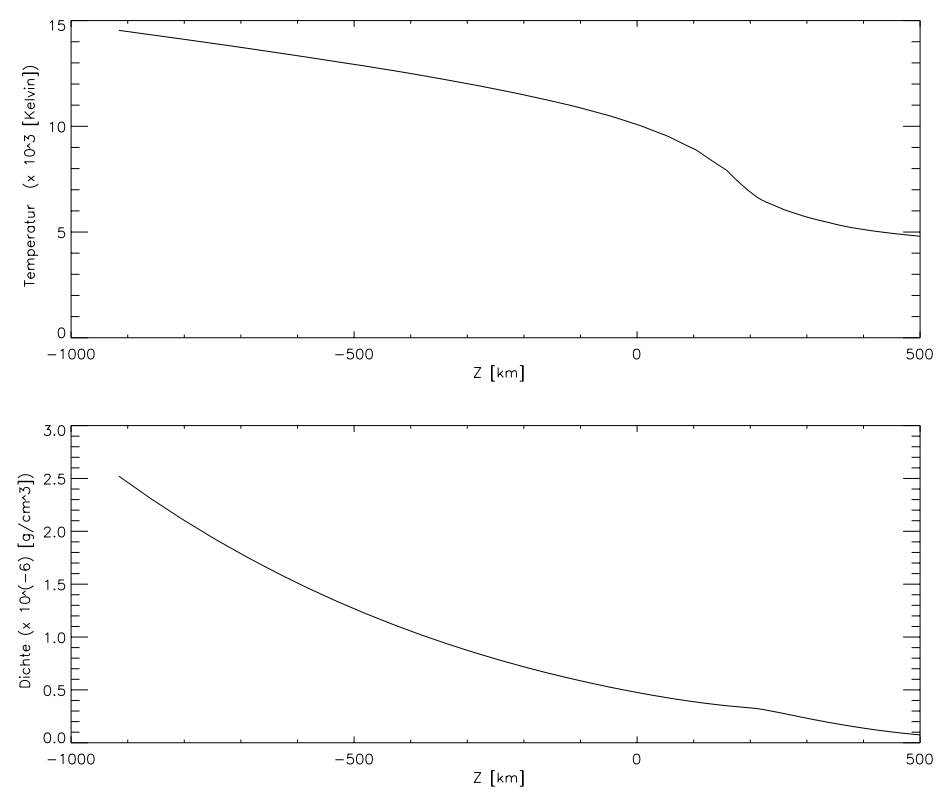

Abbildung 2.4: Anfangsbedingung für Temperatur (oben) und Massendichte (unten) $\rho$.

- Magnetfeld:

$$
\vec{B}=\left(0,0, B_{0}\right) \quad \text { mit } \quad B_{0}=100 \text { Gauss } .
$$

$-T^{b o t}$ :

$$
T^{b o t}=<T\left(z_{1}, t_{\text {hyd }}\right)>\quad .
$$

$-p^{b o t}$ :

$$
p_{\text {start }}^{\text {bot }}=<p\left(z_{1}, t_{\text {hyd }}\right)>\quad .
$$




\section{Kapitel 3}

\section{Numerische Behandlung}

Die in Kapitel 2.8 aufgeführten Gleichungen der Strahlungs-Magnetohydrodynamik bilden ein geschlossenes System gekoppelter, partieller, nichtlinearer Differentialgleichungen zur Bestimmung der Zustandsgrößen Massendichte, Impulsdichte, Magnetfeld und Energiedichte. Im Zusammenspiel mit den in Kapitel 2.9 definierten Zusatzbedingungen stellt sich ein Anfangs-Randwertproblem, welches im allgemeinen analytisch nicht lösbar ist. Zur numerischen Lösung dieses Gleichungssystems Gln. (2.46) stehen eine Vielzahl von Methoden zur Verfügung ([26], [46], [53]).

Da es aufgrund einer stochstischer Anregung in der oberen Konvektionszone und unteren Photosphäre ([54], [70], [35], [58], [78]) und durch die dynamische Wechselwirkungen zwischen Magnetfeldkonzentrationen und konvektiven Strömungen in photosphärischen Bereichen zur Erzeugung von akustischen Wellen und Schocks kommen kann, ([36], [90]) und propagierende akustische Wellen in atmosphärischen Bereichen niedrigerer Dichte zu Schockwellen aufsteilen ([96], [39]) ergeben sich folgende Forderungen an die numerische Methoden, um diese physikalischen Prozesse in adäquater Weise berücksichtigen zu können:

- Das numerische Gitter darf nicht statisch sein, sondern muß sich den Strömungen anpassen, so daß adaptive Gitterkonzepte eingesetzt werden müssen.

- Der Löser der MHD-Gleichungen (2.46) muß Schockwellen korrekt beschreiben. Dies fordert den Einsatz von Riemannlösern, die eine sehr geringe numerische Diffusivität besitzen ([106], [15]).

- Der Strahlungstransportlöser muß kleinskalige Strukturen wie Schockfronten und "scharfe" Übergänge an den Rändern von Magnetfeldkonzentrationen exakt auflösen können.

- Zur Erhöhung der Genauigkeit sollten der Strahlungstransportlöser und der MHD-Löser von höhere Konvergenzordnung in Raum und Zeit sein. 
Zur numerischen Lösung des Gleichungssystems Gln. (2.46) wurde ein 2-D MHDCode, welcher von den Herren A. Dedener und M. Wesenberg am Institut für angewandte Mathematik der Universität Freiburg (IAM) ([106], [15]) entwickelt wurde und nachfolgend aufgelisteten Eigenschaften besitzt, verwendet:

- Er arbeitet mit einem Reale-Gase-MHD-Riemannlöser auf einer FinitenVolumen-Basis und ist von 2. Konvergenzordnung in Raum und Zeit([13], [15]).

- Er arbeitet mit einer Gitteradaptionstrategie auf unstrukturierten Dreiecksgittern ([30]).

- Er ist 'shared memory' parallelisiert.

\subsection{Finite-Volumen-Verfahren auf Dreiecksgittern}

Im folgenden wird die allgemeine Struktur eines Finite-Volumen-Verfahrens für hyperbolische Erhaltungsgleichungen auf unstrukturierten Dreiecksgittern gegeben. Notation für unstrukturierte Dreiecksgitter $\mathcal{T} \in \mathbb{R}^{2}$

$T_{j}$ : Dreieck mit der Nummer $\mathbf{j}$

$T_{j l}, l=1,2,3:$ benachbarte Dreiecke von $T_{j}$;

$\left|T_{j}\right|, T_{j l}, l=1,2,3:$ Flächeninhalt der Dreiecke $T_{j l}, T_{j}$;

$S_{j l}, l=1,2,3: 1$-te Kante von $T_{j}$;

$z_{j l}:$ Mittelpunkt von $S_{j l}$;

$\vec{n}_{j l}=\left(n_{j l}^{1}, n_{j l}^{2}\right)^{T}:$ äußere Einheitsnormale an $T_{j}$ im Punkt $z_{j l}$;

$\vec{U}_{j}^{n}$ : im Dreieck $T_{j}$ konstante Approximation der exakten Lösung;

$\vec{U}_{j l}^{n}$ : im Dreieck $T_{j l}$ konstante Approximation der exakten Lösung;

Integration der Gln. (2.46) mit den physikalischen Flüssen Gl.(2.47) über das Dreieck $T_{j}$ liefert:

$$
\int_{T_{j}} \partial_{t} \vec{U}(t)=-\int_{T_{j}}\left[\partial_{x} \vec{F}(\vec{U})+\partial_{z} \vec{G}(\vec{U})+\vec{Q}(\vec{U})\right]
$$

Unter Verwendung des Integralsatz von Gauß folgt:

$$
\int_{T_{j}} \partial_{t} \vec{U}(t)=-\int_{\partial T_{j}}\left(\begin{array}{c}
\vec{F}(\vec{U}) \\
\vec{G}(\vec{U})
\end{array}\right) \cdot \vec{n}+\int_{T_{j}} \vec{Q}(\vec{U})
$$


Mit den Mittelwerten von $\vec{U}$ und $\vec{Q}(\vec{U})$ auf $T_{j}$

$$
\begin{aligned}
\vec{U}_{j}(t) & =\frac{1}{\left|T_{j}\right|} \int_{T_{j}} \vec{U}(\vec{r}, t), \\
\vec{Q} & =\frac{1}{\left|T_{j}\right|} \int_{T_{j}} \vec{Q}(\vec{r})
\end{aligned}
$$

als Approximation der exakten Lösung $\vec{U}$ und $\vec{Q}$ auf dem Dreieck $T_{j}$ und

$$
\sum_{l=1}^{3} g_{j l}\left(\vec{U}_{j}^{n}, \vec{U}_{j l}^{n}\right) \equiv\left(\begin{array}{c}
\vec{F}\left(\vec{U}_{j}^{n}\right) \\
\vec{G}\left(\vec{U}_{j}^{n}\right)
\end{array}\right) \cdot \sum_{l=1}^{3}\left|S_{j l}\right| \vec{n}_{j l}
$$

folgt folgende Form für Gl. (3.2):

$$
\partial_{t} \vec{U}_{j}=-\frac{1}{\left|T_{j}\right|} \sum_{l=1}^{3} g_{j l}\left(\vec{U}_{j}^{n}, \vec{U}_{j l}^{n}\right)+\vec{Q}(\vec{U})
$$

Unter Approximation der Zeitableitung durch den Differentialquotienten 1.Ordnung ergibt sich das Finite-Volumen-Verfahren 1.Ordnung zur Lösung des AnfangsRandwertproblem bestehend aus den zweidimensionalen MHD-Gleichungen GIn. (2.46) und den in Kap. 2.9 beschriebenen Anfangs- und Randbedingungen, im Falle unstrukturierter Dreiecksgitter, in der Form:

$$
\vec{U}_{j}^{n+1}=\vec{U}_{j}^{n}-\frac{\Delta t_{n}}{\left|T_{j}\right|} \sum_{l=1}^{3} g_{j l}\left(\vec{U}_{j}^{n}, \vec{U}_{j l}^{n}\right)+\Delta t_{n} \vec{Q}_{j}\left(\vec{U}_{j}^{n}\right)
$$

\subsection{Godunov-Methode - Riemannlöser}

Die Godunov-Methode wurde von Godunov 1959 entwickelt ([34]) um die Euler(HD)Gleichungen im Falle von Schockwellen numerisch zu lösen. Dazu schlug er vor lokale Riemannprobleme zu behandeln. Im Folgenden werden nun die Grundlagen der Godunov-Methode im Falle eine eindimensionalen Riemannproblems kurz erläutert ([98]). Ausgehend von der allgemeinen Darstellung eines Anfangs-Randwertproblem für nichtlineare Erhaltungsgleichungen

$$
\begin{array}{r}
\partial_{t} \vec{U}+\partial_{x} \vec{F}(\vec{U})=0 \\
\vec{U}(x, 0)=\vec{U}^{(0)}(x) \\
\vec{U}(0, t)=\vec{U}_{L}(t), \vec{U}(L, t)=\vec{U}_{R}(t)
\end{array}
$$

$\left(\vec{U}(x, t)\right.$ : Vektor konservativer Variablen, $\vec{F}(\vec{U})$ : Flußvektor, $\vec{U}^{(0)}(x)$ : Anfangsdaten zur Zeit $t=0,[0, L]$ : Gebietsintervall, $\vec{U}_{R}(t), \vec{U}_{L}(t):$ Randbedingungen) 
und ihrer Integralform für ein beliebiges Kontrollvolumen $\left[x_{1}, x_{2}\right] \times\left[t_{1}, t_{2}\right]$ :

$$
\begin{gathered}
\int_{x_{1}}^{x_{2}} \vec{U}\left(x, t_{2}\right) d x=\int_{x_{1}}^{x_{2}} \vec{U}\left(x, t_{1}\right) d x+\int_{t_{1}}^{t_{2}} \vec{F}\left(\vec{U}\left(x_{1}, t\right)\right) d t \\
-\int_{t_{1}}^{t_{2}} \vec{F}\left(\vec{U}\left(x_{2}, t\right)\right) d t
\end{gathered}
$$

fordert die Godunov-Methode einen stückweise konstanten Verlauf der Variablen pro Kontrollvolumen. Dadurch kann die diskrete Darstellung der zeitlichen Entwicklung einer Lösung $\widetilde{\vec{U}}\left(x, t^{n}\right)$ vom Zeitpunkt $t=t^{n}$ zu einem Zeitpunkt $t^{n+1}=$ $t^{n}+\Delta t$ in dem durch $M$ Gitterzellen diskretisierten Rechengebiet ermittelt werden. Zum Zeitpunkt $t^{n+1}$ haben die Zellenmittelwerte folgende Form:

$$
\vec{U}_{i}^{n+1}=\frac{1}{\Delta x} \int_{x_{i-\frac{1}{2}}}^{x_{i+\frac{1}{2}}} \tilde{\vec{U}}\left(x, t^{n+1}\right) d x
$$

Danach wird das ursprüngliche Anfangs-Randwertproblem auf der Basis der ermittelten Zellmittelwerte gelöst. Es entstehen an den Verbindungspunkten benachbarter Gitterzellen, welche die Position $x_{i+\frac{1}{2}}$ und $x_{i-\frac{1}{2}}$ haben, lokale Riemannprobleme $R P\left(\vec{U}_{i}^{n}, \vec{U}_{i+1}^{n}\right), R P\left(\vec{U}_{i-1}^{n}, \vec{U}_{i}^{n}\right)$ (siehe Abb. 3.1).

Die Godunov-Methode läßt sich nun folgendermaßen darstellen:

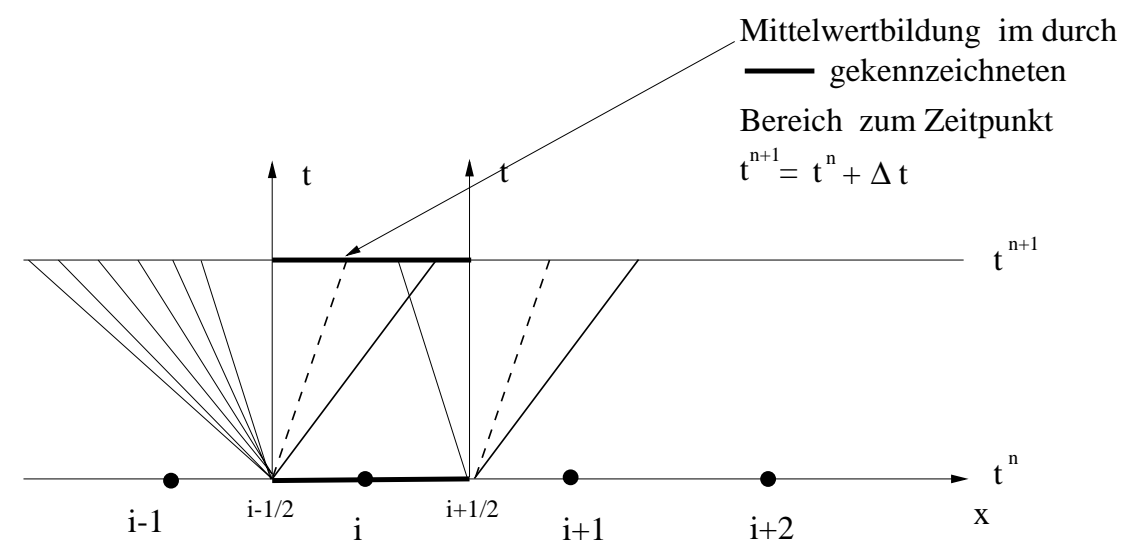

Abbildung 3.1: Typische Charakteristiken als Lösungen von lokalen Riemannproblemen an den Grenzen benachbarter Kontrollvolumina $x_{i+\frac{1}{2}}$ und $x_{i-\frac{1}{2}}$ und die speziell gekennzeichneten Bereiche in denen die Godunov-Mittelung der lokalen Riemannlösungen innerhalb einer Zelle zum Zeitpunkt $t^{n+1}$ durchgeführt wird ([98]).

$$
\vec{U}_{i}^{n+1}=\vec{U}_{i}^{n}+\frac{\Delta t}{\Delta x}\left[\vec{F}_{i-\frac{1}{2}}^{n}-\vec{F}_{i+\frac{1}{2}}^{n}\right]
$$


wobei für den Fall, daß $\Delta t \leq \frac{\Delta x}{S_{\max }^{n}}\left(S_{\max }^{n}\right.$ : maximale Charakteristikengeschw. in der betrachteten Gitterzelle) gilt, die numerischen Flüsse gegeben sind durch:

$$
\vec{F}_{i+\frac{1}{2}}^{n}=\vec{F}\left(\vec{U}_{i+\frac{1}{2}}^{n}(0)\right)=\frac{1}{\Delta t} \int_{t^{n}}^{t^{n+1}} \vec{F}\left(\vec{U}_{i+\frac{1}{2}}^{n}(t)\right) d t
$$

Dabei ist $\vec{U}_{i+\frac{1}{2}}^{n}(0)$ die Lösung des Riemannproblems $R P\left(\vec{U}_{i}^{n}, \vec{U}_{i+1}^{n}\right)$ entlang der Geraden $x / t=0$ (parallel zur $t$-Achse). Wie in Kap. 3.1 verdeutlicht beinhaltet die Formulierung des zweidimensionalen Anfangs-Randwertproblems der MHDGleichungen im Finite-Volumen-Kontext die Bildung von sogenannten Zellmittelwerten, welche auch eine Notwendigkeit beim Einsatz numerischer Methoden des Godunov-Typs sind. Die Bestimmung der korrespondierenden numerischen Flüsse, die in Gl. (3.15) für den eindimensionalen Fall dargestellt sind, im Falle von Dreiecksgittern, erfolgt ganz analog für jede der drei Dreieckskanten.

Zur Lösung des Gleichungssystems Gln. (2.46) sind zwei nachfolgend näher erläuterte MHD-Riemann-Löser vom Godunov-Typ in einer Art Hybridschema eingesetzt worden.

- Der von Dai und Woodward entwickelte und auf der Charakteristikendarstellung basierender MHD-Riemann-Löser, bei dem die numerischen Interzellflüsse aus den charakteristischen Gleichungen und den korrespondierenden generalisierten Riemanninvarianten ermittelt werden. Dieser Löser ist sehr wenig diffusiv, weshalb er zu Stabilitätsproblemen führen kann ([105]).

- Der von Harten, Lax und Van Leer (HLL) entwickelte Löser ([37]) bei dem eine direkte Approximation für die numerischen Interzellflüsse entwickelt wurde. Dieser Löser ist im Gegensatz zu ersterem erheblich diffusiver ([105]).

\subsection{Konservative-Primitive Variablen}

Die konservative Formulierung der MHD-Gleichung in einer Raumdimension lautet:

$$
\partial_{t} \vec{U}+\partial_{x} \vec{F}(\vec{U})=0
$$

mit

$$
\begin{aligned}
\vec{U}:=\vec{U}(\vec{r}, t) & :=(\rho, \rho \vec{v}, \vec{B}, E)^{T} \\
& =\left(\rho, \rho v_{x}, \rho v_{y}, \rho v_{z}, B_{x}, B_{y}, B_{z}, E\right)^{T}
\end{aligned}
$$


und

$$
\vec{F}(\vec{U}):=\left(\begin{array}{c}
\rho v_{x} \\
\rho v_{x}^{2}+p+\frac{\vec{B}^{2}}{8 \pi}-\frac{B_{x}^{2}}{4 \pi} \\
0 \\
\rho v_{x} v_{z}-\frac{B_{z} B_{x}}{4 \pi} \\
0 \\
0 \\
v_{x} B_{z}-v_{z} B_{x} \\
\left(\rho e+p+\frac{\vec{B}^{2}}{8 \pi}\right)-\frac{B_{x}}{4 \pi} \vec{B} \cdot \vec{v}
\end{array}\right)
$$

Zur Ermittelung der numerischen Flußfunktionen reicht es aus nur ein 1D-System zu betrachten, denn das betrachtete Gleichungssystem ist rotationsinvariant([106], [98]), weshalb die Bestimmung der numerischen Flüße in x- und z-Richtung äquivalent ist. Das System von nichtlinearen Erhaltungsgleichungen läßt sich auch in der der Gl. 3.16 äquivalenten quasi-linearen Form dargestellen:

$$
\partial_{t} \vec{U}+\mathcal{A}(\vec{U}) \partial_{x} \vec{U}=0
$$

Dabei entspricht die Koeffizientenmatrix $\mathcal{A}(\vec{U})$ der Jakobimatrix ([106]) und hat folgende Form:

$$
\begin{gathered}
\mathcal{A}(\vec{U}):= \\
\left(\begin{array}{ccccccc}
0 & 1 & 0 & 0 & 0 & 0 & 0 \\
\frac{\gamma-3}{2} v_{x}^{2}+\frac{\gamma-1}{2}\left(v_{x}^{2}+v_{z}^{2}\right) & (3-\gamma) v_{x} & (1-\gamma) v_{y} & (1-\gamma) v_{z} & \frac{2-\gamma}{4 \pi_{1}} B_{y} & \frac{2-\gamma}{4 \pi} B_{z} & \gamma-1 \\
-v_{x} v_{y} & v_{y} & v_{x} & 0 & -\frac{1}{4 \pi} B_{x} & 0 & 0 \\
-v_{x} v_{z} & v_{z} & 0 & v_{x} & 0 & -\frac{1}{4 \pi} B_{x} & 0 \\
\frac{v_{y} B_{x}-v_{x} B_{y}}{\rho} & \frac{B_{y}}{\rho} & -\frac{B_{x}}{\rho} & 0 & v_{x} & 0 & 0 \\
\frac{v_{z} B_{x}-v_{x} B_{z}}{\rho} & \frac{B_{z}}{\rho} & 0 & -\frac{B_{x}}{\rho} & 0 & v_{x} & 0 \\
\alpha_{1} & \alpha_{2} & \alpha_{3} & \alpha_{4} & \alpha_{5} & \alpha_{6} & \alpha_{7}
\end{array}\right)
\end{gathered}
$$

mit

$$
\begin{aligned}
& \alpha_{1}=v_{x}\left(\frac{\gamma-1}{2} \vec{u}^{2}-\frac{1}{\rho}\left(\rho E+p+\frac{B_{y}^{2}+B_{z}^{2}}{8 \pi}\right)\right)+\frac{B_{x}}{4 \pi \rho}\left(v_{y} B_{y}+v_{z} B_{z}\right), \\
& \alpha_{2}=\frac{1}{\rho}\left(\rho E+p+\frac{B_{y}^{2}+B_{z}^{2}}{8 \pi}\right)+(1-\gamma) v_{x}^{2}, \\
& \alpha_{3}=(1-\gamma) v_{x} v_{y}-\frac{B_{x} B_{y}}{4 \pi \rho}, \\
& \alpha_{4}=(1-\gamma) v_{x} v_{z}-\frac{B_{x} B_{z}}{4 \pi \rho}, \\
& \alpha_{5}=\frac{2-\gamma}{4 \pi} v_{x} B_{y}-\frac{1}{4 \pi} v_{y} B_{x}, \\
& \alpha_{6}=\frac{2-\gamma}{4 \pi} v_{x} B_{z}-\frac{1}{4 \pi} v_{z} B_{x}, \\
& \alpha_{7}=\gamma v_{x} .
\end{aligned}
$$


Zur Bestimmung von Eigenwerten und Eigenvektoren der Jakobimatrix $\mathcal{A}(\vec{W})$ bietet es sich an das quasilineare System der vereinfachten 1D-Gleichungen Gl. (3.18) in primitiven Variablen mit dem Variablenvektor

$$
\vec{W}:=(\tau, \vec{v}, \vec{B}, p)^{T}
$$

$\left(\tau:=\frac{1}{\rho}\right)$ zu formulieren, da sich dadurch die Eigenwerte und die Rechts- und Linkseigenvektoren einfacher berechnen lassen. Die MHD-Gleichungen schreiben sich dann zu

$$
\partial_{t} \vec{W}+\mathcal{A}(\vec{W}) \partial_{x} \vec{W}=0
$$

mit

$$
\mathcal{A}(\vec{W}):=\frac{\partial \vec{W}}{\partial \vec{U}} \mathcal{A}(\vec{U}) \frac{\partial \vec{U}}{\partial \vec{W}}\left(\begin{array}{ccccccc}
v_{x} & -\tau & 0 & 0 & 0 & 0 & 0 \\
0 & v_{x} & 0 & 0 & \frac{1}{4 \pi} B_{y} \tau & \frac{1}{4 \pi} B_{z} \tau & \tau \\
0 & 0 & v_{x} & 0 & -\frac{1}{4 \pi} B_{x} \tau & 0 & 0 \\
0 & 0 & 0 & v_{x} & 0 & -\frac{1}{4 \pi} B_{x} \tau & 0 \\
0 & B_{y} & -B_{x} & 0 & v_{x} & 0 & 0 \\
0 & B_{z} & 0 & -B_{x} & 0 & v_{x} & 0 \\
0 & \gamma p & 0 & 0 & 0 & 0 & v_{x}
\end{array}\right) .
$$

Für glatte Lösungen sind die konservative und die primitive Formulierung äquivalent. Im Falle von Schockwellen kann jedoch die Wellengeschwindigkeit unkorrekt sein, da die Erhaltungseigenschaften verletzt werden. Aus diesem Grunde wird das in primitiven Variablen formulierte Riemannproblem des nichtlinearen Systems durch das, des linearen Systems approximiert. Die Koeffizientenmatrizen $\mathcal{A}(\vec{U})$ und $\mathcal{A}(\vec{W})$ sind vom jeweiligen Variablenvektor unabhängig. Nachfolgend sind nun die Eigenwerte der Matrix $\mathcal{A}(\vec{W})$ und die den Eigenwerten zugeordneten Wellen erläutert (Abb. 3.2):

$$
\begin{aligned}
\lambda_{f_{ \pm}} & =v_{x} \pm v_{f} \quad(\text { schnelle magnetoakustische Wellen) } \\
\lambda_{a_{ \pm}} & =v_{x} \pm v_{a x} \quad(\text { Alfvén-Wellen) } \\
\lambda_{s_{ \pm}} & =v_{x} \pm v_{s} \quad \text { (langsame magnetoakustische Wellen) } \\
\lambda_{0} & =v_{x} \quad \text { (Entropiewelle, Kontaktdiskontinuität) },
\end{aligned}
$$


wobei die nichtnegativen Geschwindigkeiten $v_{f}, v_{a x}$ und $v_{s}$ durch

$$
\begin{aligned}
v_{a x}^{2} & :=\tau \frac{B_{x}^{2}}{4 \pi}, \\
c_{s}^{2} & :=\gamma p \tau, \\
v_{a}^{2} & :=\tau \frac{B_{x}^{2}+B_{y}^{2}+B_{z}^{2}}{4 \pi}, \\
v_{f}^{2} & :=\frac{1}{2}\left[\left(v_{a}^{2}+c_{s}^{2}\right)+\sqrt{\left(v_{a}^{2}+c_{s}^{2}\right)^{2}-4 v_{a x}^{2} c_{s}^{2}}\right], \\
v_{f}^{2} & :=\frac{1}{2}\left[\left(v_{a}^{2}+c_{s}^{2}\right)-\sqrt{\left(v_{a}^{2}+c_{s}^{2}\right)^{2}-4 v_{a x}^{2} c_{s}^{2}}\right]
\end{aligned}
$$

gegeben sind.

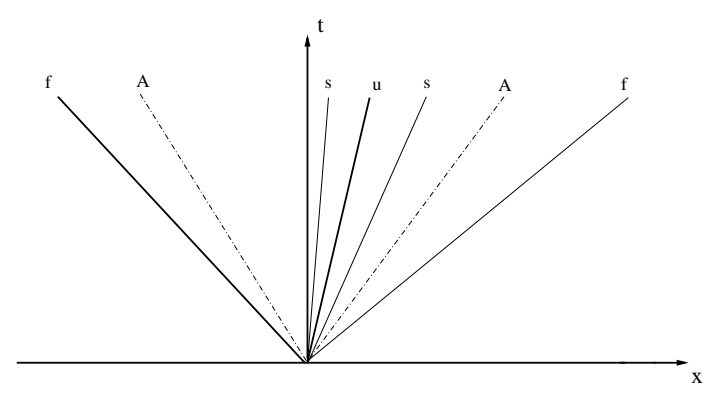

Abbildung 3.2: Charakteristiken für die idealen MHD-Gleichungen als Lösungen eines Riemannproblems. Die Symbole f, A, s bezeichnen schnelle, Alfvén und langsame Wellen. u kennzeichnet die Kontaktdiskontinuität oder Entropiewelle.

Die zugehörigen Rechtseigenvektoren sind

$$
\begin{aligned}
& r_{0}=\left(\begin{array}{l}
\tau \\
0 \\
0 \\
0 \\
0 \\
0 \\
0
\end{array}\right) \quad, \quad r_{ \pm v_{a x}}=\frac{v_{f}}{\sqrt{2}}\left(\begin{array}{c}
0 \\
0 \\
\pm \beta_{z} \\
\pm \beta_{y} \\
-\operatorname{sgn}\left(B_{x}\right) \sqrt{\frac{4 \pi}{\tau}} \beta_{z} \\
\operatorname{sgn}\left(B_{x}\right) \sqrt{\frac{4 \pi}{\tau}} \beta_{y} \\
0
\end{array}\right) \\
& r_{ \pm v_{f}}=R_{ \pm v_{f}}\left(\begin{array}{c}
\mp \alpha_{f} \tau \\
\alpha_{f} v_{f} \\
-\alpha_{s} \beta_{y} v_{a x} \operatorname{sgn}\left(B_{x}\right) \\
\pm \alpha_{s} \beta_{y} v_{f} \sqrt{\frac{4}{\pi}} \tau \\
\pm \alpha_{s} \beta_{z} v_{f} \sqrt{\frac{4}{\pi}} \tau \\
\pm \alpha_{f} \gamma p
\end{array}\right) \\
& r_{ \pm v_{s}}=R_{ \pm v_{s}}\left(\begin{array}{c}
\mp \alpha_{s} \tau \\
\alpha_{s} v_{s} \\
-\alpha_{f} \beta_{y} c_{s} s g n\left(B_{x}\right) \\
-\alpha_{s} \beta_{z} c_{s} \operatorname{sgn}\left(B_{x}\right) \\
\mp \alpha_{f} \beta_{y} \frac{c_{s}^{2}}{v_{f}} \sqrt{\frac{4}{\pi}} \tau \\
\mp \alpha_{f} \beta_{z} \frac{c_{s}^{2}}{v_{f}} \sqrt{\frac{4}{\pi}} \tau \\
\pm \alpha_{s} \gamma p
\end{array}\right)
\end{aligned}
$$




$$
\begin{aligned}
& \alpha_{f}:=\left\{\begin{array}{rll}
\frac{\sqrt{v_{f}^{2}-v_{a x}^{2}}}{\sqrt{v_{f}^{2}-v_{s}^{2}}} & : & \left(v_{f}^{2}>v_{s}^{2}\right) \\
1 & : & \left(v_{f}^{2}=v_{s}^{2}\right)
\end{array} \quad, \quad \beta_{y}:=\left\{\begin{array}{rll}
\frac{B_{y}}{\sqrt{B_{y}^{2}+B_{z}^{2}}} & : & \left(B_{y}^{2}+B_{z}^{2}>0\right) \\
\frac{1}{\sqrt{2}} & : & \left(B_{y}^{2}+B_{z}^{2}>0\right)
\end{array},\right.\right. \\
& \alpha_{s}:=\left\{\begin{array}{rll}
\frac{\sqrt{v_{f}^{2}-c_{s}^{2}}}{\sqrt{v_{f}^{2}-v_{s}^{2}}} & : & \left(v_{f}^{2}>v_{s}^{2}\right) \\
0 & : & \left(v_{f}^{2}=v_{s}^{2}\right)
\end{array} \quad, \beta_{y}:=\left\{\begin{array}{rll}
\frac{B_{z}}{\sqrt{B_{y}^{2}+B_{z}^{2}}} & : & \left(B_{y}^{2}+B_{z}^{2}>0\right) \\
\frac{1}{\sqrt{2}} & : & \left(B_{y}^{2}+B_{z}^{2}>0\right)
\end{array}\right.\right. \\
& R_{ \pm v_{f}}:=\frac{v_{f}}{\sqrt{\alpha_{f}^{2}\left(v_{f}^{2}+c_{s}^{2}\right)+\alpha_{s}^{2}\left(v_{f}^{2}+v_{a x}^{2}\right)}} \text {, } \\
& R_{ \pm v_{s}}:=\frac{v_{f}^{2}}{\sqrt{\alpha_{f}^{2} c_{s}^{2}\left(v_{f}^{2}+c_{s}^{2}\right)+\alpha_{s}^{2} v_{f}^{2}\left(v_{f}^{2}+c_{s}^{2}\right)}} \\
& \operatorname{sgn}\left(B_{x}\right):=\left\{\begin{array}{rll}
1 & : & \left(B_{x} \geq 0\right) \\
-1 & : & \left(B_{x}<0\right)
\end{array} \quad .\right.
\end{aligned}
$$

Die zugehörigen Linkseigenvektoren lauten:

$$
\begin{aligned}
\vec{L}_{0}= & \left(\frac{1}{\tau}, 0,0,0,0,0, \frac{1}{\gamma p}\right), \\
\vec{L}_{ \pm v_{a x}}= & \left.\frac{1}{\sqrt{2} v_{f}}\left(0,0, \pm \beta_{z}, \mp \beta_{y},-\operatorname{sgn}\left(B_{x}\right) \frac{\beta_{z}}{\sqrt{4 \pi}} \sqrt{\tau}, \operatorname{sgn}\left(B_{x}\right) \frac{\beta_{y}}{\sqrt{4 \pi}} \sqrt{\tau}, 0\right)\right), \\
\vec{L}_{ \pm v_{a x}}= & \frac{R_{ \pm v_{f}}}{v_{f}^{2}}\left(0, \alpha_{f} v_{f},-\alpha_{s} \beta_{y} v_{a x} \operatorname{sgn}\left(B_{x}\right),-\alpha_{s} \beta_{z} v_{a x} \operatorname{sgn}\left(B_{x}\right), \pm \frac{\alpha_{s} \beta_{y} v_{f}}{\sqrt{4 \pi}} \sqrt{\tau},\right. \\
& \left. \pm \frac{\alpha_{s} \beta_{z} v_{f}}{\sqrt{4 \pi}} \sqrt{\tau}, \pm \alpha_{f} \tau\right), \\
\vec{L}_{ \pm v_{a x}}= & \frac{R_{ \pm v_{s}}}{v_{f}^{2}}\left(0, \alpha_{s} v_{s}, \alpha_{f} \beta_{y} c_{s} \operatorname{sgn}\left(B_{x}\right), \alpha_{f} \beta_{z} c_{s} \operatorname{sgn}\left(B_{x}\right), \mp \frac{\alpha_{f} \beta_{y}}{4 \pi} \frac{c_{s}^{2}}{v_{f}} \sqrt{\tau},\right. \\
& \left.\mp \frac{\alpha_{f} \beta_{z}}{4 \pi} \frac{c_{s}^{2}}{v_{f}} \sqrt{\tau}, \pm \alpha_{s} \tau\right) .
\end{aligned}
$$

\subsection{Dai-Wodward-Löser}

Beim Dai-Wodward-Löser ([10]) wird nun der entsprechend verallgemeinerte numerische Fluß Gl. (3.15) aus den charakteristischen Gleichungen und den korrespondierenden generalisierten Riemanninvarianten approximativ bestimmt. Dazu ist die Bestimmung des in Gln. (3.13) dargestellten Zellenmittelwertes $\overrightarrow{\vec{W}}$, im 'primitiven Bild', unter Zuhilfenahme der Gln. (3.31) notwendig. Mit $\vec{W}_{0}^{k}:=\frac{\left(\vec{W}_{L}+\vec{W}_{R}\right)}{2}$ läßt sich der Zellenmittelwert $\overrightarrow{\vec{W}}$ als Lösung des folgenden Gleichungssystems ermitteln,

$$
\vec{L}_{k} \cdot\left(\vec{W}-\vec{W}_{0}^{k}\right)=0 \quad(1 \leq k \leq 7)
$$


welches aus der Tatsache folgt, daß entlang einer Charakteristik $\left(d x / d t=\lambda_{i} \quad(i=\right.$ $1, \ldots, 7)$ )

$$
\vec{L}_{k} \cdot d \vec{W}=0
$$

gilt ([106]).

Der sich daraus für den eindimensionalen Fall an der Stelle $x_{i+\frac{1}{2}}$ ergebende numerische Fluß Gl. (3.15) hat folgende Form ([98], [10]):

$$
\begin{aligned}
F_{i+\frac{1}{2}}^{D W}=F\left(\vec{W}_{i+\frac{1}{2}}(0)\right) & :=F(\overrightarrow{\vec{W}}) \\
& +\min \left(c\left|\vec{W}_{L}-\vec{W}_{R}\right|, 1\right)\left(\vec{W}_{L}-\vec{W}_{R}\right)
\end{aligned}
$$

$(c:=0.05)$.

\subsection{HLL-Löser}

Das Problem bei der numerischen Behandlung von Riemannproblemen mit Verfahren vom Godunovtyp liegt bei der Ermittelung der durch Gl. (3.13) dargestellten Zellenmittelwerte und den daraus resultierenden Godunov-Flüssen Gl. (3.15). Harten, Lax und van Leer entwickelten einen Löser ([37]), wobei sie ausgehend von der in Abb. 3.2 dargestellten Wellenstruktur der exakten Lösung des MHDRiemannproblems ein Kontrollvolumen $\left[x_{L}, x_{R}\right] \times\left[t_{1}, t_{2}\right]$ definierten, welches die gesamte Wellenstruktur beinhaltet (siehe Abb. 3.3). Dabei gilt mit $S_{L}$ und $S_{R}$, den schnellsten Signalgeschwindigkeiten:

$$
x_{L} \leq t_{2} S_{L}, \quad x_{R} \geq t_{2} S_{R},
$$

welche nach S.F. Davis (1984) ([11]) folgendermaßen abgeschätzt werden: $S_{L}=$ $u_{l}-a_{l}$ und $S_{R}=u_{r}-a_{r}$. Dabei sind $u_{l}$ und $u_{r}$ die Zellenmittelwerte der Strömungsgeschwindigkeiten, der beiden Zellen zwischen denen das Riemannproblem besteht. $a_{l}:=\sqrt{\gamma_{l} \frac{p_{l}}{\rho_{l}}}, a_{r}:=\sqrt{\gamma_{r} \frac{p_{r}}{\rho_{r}}}, p_{l}, p_{r}, \rho_{l}, \rho_{r}, \gamma_{l}$ und $\gamma_{r}$ sind die in diesen Zellen bestehenden Schallgeschwindigkeiten, Drücke, Massendichten und Adiabatenexponenten.

Die Integralform Gl. (3.11) der mit den primitiven Variablen formulierten Erhaltungsgleichungen erhält dann folgende Form:

$$
\begin{aligned}
\int_{x_{L}}^{x_{R}} \vec{W}\left(x, t_{2}\right) d x & =\int_{x_{L}}^{x_{R}} \vec{W}\left(x, t_{1}\right) d x+\int_{t_{1}}^{t_{2}} \vec{F}\left(\vec{W}\left(x_{L}, t\right)\right) d t-\int_{t_{1}}^{t_{2}} \vec{F}\left(\vec{W}\left(x_{R}, t\right)\right) d t \\
& =x_{R} \vec{W}_{R}-x_{L} \vec{W}_{L}+t_{2}\left(\vec{F}\left(\vec{W}\left(x_{L}\right)\right)-\vec{F}\left(\vec{W}\left(x_{R}\right)\right)\right)
\end{aligned}
$$




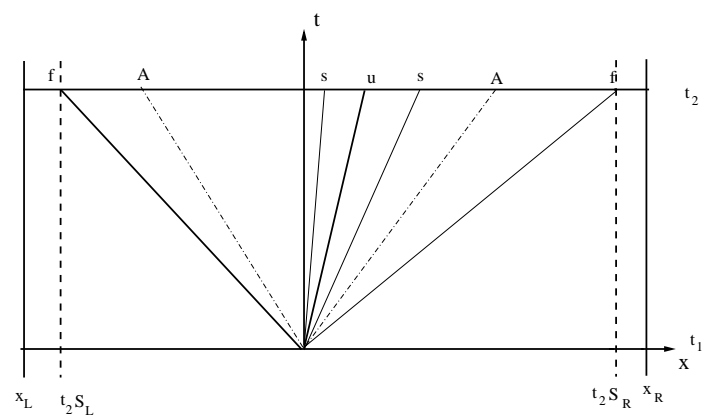

Abbildung 3.3: Das Kontrollvolumen $\left[x_{L}, x_{R}\right] \times\left[t_{1}, t_{2}\right]$ enthält die Charakteristiken der Lösung eines MHD-Riemannproblems. $S_{L}$ und $S_{R}$ sind die schnellsten Signalgeschwindigkeiten dieses Riemannproblems.

Eine Aufspaltung des Integrals auf der linken Seite ergibt:

$$
\begin{aligned}
\int_{x_{L}}^{x_{R}} \vec{W}\left(x, t_{2}\right) d x=\int_{x_{L}}^{t_{2} S_{L}} \vec{W}\left(x, t_{2}\right) d x & +\int_{t_{2} S_{L}}^{t_{2} S_{R}} \vec{W}\left(x, t_{2}\right) d t \\
& +\int_{t_{2} S_{R}}^{x_{R}} \vec{W}\left(x, t_{2}\right) d t
\end{aligned}
$$

und einige Umformungen liefern:

$$
\begin{aligned}
\int_{x_{L}}^{x_{R}} \vec{W}\left(x, t_{2}\right) d x=\int_{t_{2} S_{L}}^{t_{2} S_{R}} \vec{W}\left(x, t_{2}\right) d x & +\left(t_{2} S_{L}-x_{L}\right) \vec{W}_{L} \\
& +\left(x_{R}-t_{2} S_{R}\right) \vec{W}_{R} .
\end{aligned}
$$

Der Vergleich von Gl. (3.35) und Gl. (3.37) liefert:

$$
\begin{aligned}
\int_{t_{2} S_{L}}^{t_{2} S_{R}} \vec{W}\left(x, t_{2}\right) d t=t_{2}\left(S_{R} \vec{W}_{R}-S_{L} \vec{W}_{L}\right. & +\vec{F}\left(\vec{W}\left(x_{L}\right)\right) \\
& \left.-\vec{F}\left(\vec{W}\left(x_{R}\right)\right)\right) .
\end{aligned}
$$

Das Integralmittel der exakten Riemannlösung zwischen der langsamsten und der schnellsten Ausbreitungscharakteristik, erhält man durch Division von Gl. (3.38) durch die Länge $t_{2}\left(S_{R}-S_{L}\right)$ :

$$
\begin{array}{r}
\vec{W}^{h l l}=\frac{1}{t_{2}\left(S_{R}-S_{L}\right)} \int_{t_{2} S_{L}}^{t_{2} S_{R}} \vec{W}\left(x, t_{2}\right) d t \\
=\frac{S_{R} \vec{W}_{R}-S_{L} \vec{W}_{L}+\vec{F}\left(\vec{W}\left(x_{L}\right)\right)-\vec{F}\left(\vec{W}\left(x_{R}\right)\right)}{S_{R}-S_{L}} .
\end{array}
$$

Harten, Lax und van Leer schlugen nun folgenden approximativen Riemannlöser 
vor

$$
\tilde{\vec{W}}(x, t)=\left\{\begin{array}{rll}
\vec{W}_{L} & : & \frac{x}{t} \leq S_{L} \\
\vec{W}^{h l l} & : & S_{L} \leq \frac{x}{t} \leq S_{R} \\
\vec{W}_{R} & : & \frac{x}{t} \geq S_{R}
\end{array} .\right.
$$

Der Godunov-Fluß Gl. (3.15) entlang der t-Achse läßt sich durch Betrachtung des

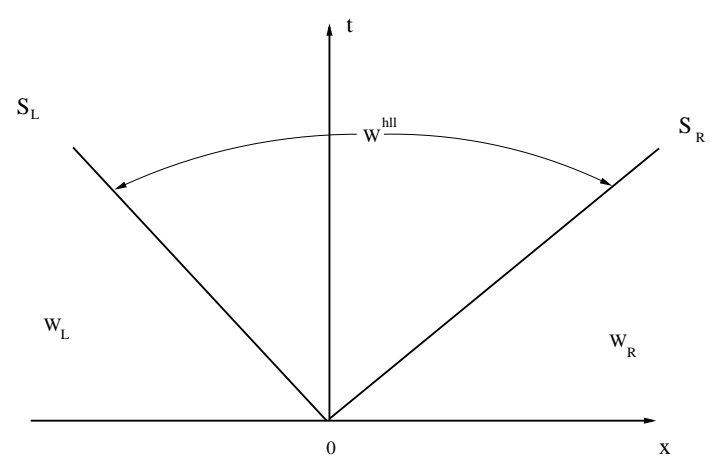

Abbildung 3.4: Approximativer Riemannlöser. Die Lösung in dem Bereich, der von der langsamsten und der schnellsten Welle bestimmt wird, besteht aus einem einzigen durch $\vec{W}^{\text {hll }}$ beschriebenen Zustand.

halben Kontrollvolumens $\left[x_{L}, 0\right] \times\left[t_{1}, t_{2}\right]$ oder $\left[0, x_{R}\right] \times\left[t_{1}, t_{2}\right]$ ermitteln:

$$
\begin{aligned}
\vec{F}^{h l l}\left(\vec{W}_{i+\frac{1}{2}}(0)\right) & =\vec{F}_{0 L}\left(\vec{W}_{i-\frac{1}{2}}(0)\right) \\
& =\vec{F}_{L}-S_{L} W_{L}-\frac{1}{t_{2}} \int_{T S_{L}}^{0} \vec{W}\left(x, t_{2}\right) d x \\
& =\vec{F}_{R}-S_{R} W_{R}-\frac{1}{t_{2}} \int_{T S_{R}}^{0} \vec{W}\left(x, t_{2}\right) d x=\vec{F}_{0 R}\left(\vec{W}_{i+\frac{1}{2}}(0)\right) .
\end{aligned}
$$

Die Einbeziehung von Gl. (3.40) liefert die entgültige Form:

$$
\vec{F}_{i+\frac{1}{2}}^{h l l}=\left\{\begin{array}{cl}
\vec{F}_{L} & : \quad 0 \leq S_{L} \\
\frac{S_{R} \vec{F}_{L}-S_{L} \vec{F}_{R}+S_{L} S_{R}\left(\vec{W}_{R}-\vec{W}_{L}\right)}{S_{R}-S_{L}} & : \quad \text { falls } S_{L} \leq \frac{x}{t} \leq S_{R} . \\
\vec{F}_{R} & : \quad 0 \geq S_{R}
\end{array}\right.
$$

Dieser Löser ist als sogenannter Zweiwellenlöser sehr diffusiv. Da der von Dai und Woodward entwickelte Löser so gut wie gar nicht diffusiv ist und daher Stabilitätsprobleme bekommen kann, die sich in negativen Drücken widerspiegeln, wird in einem solchen Fall auf den Hll-Löser umgeschaltet ([105]). 


\subsection{Riemannlöser für partiell ionisierte Gase}

Da im Fall eines idealen partiell ionisierten Gases die kalorische Zustandsgleichung

$$
\epsilon(T, \rho)=\rho c_{v} T
$$

$\left(\epsilon(T, \rho)\right.$ : totale innere Energiedichte, $\rho$ : Massendichte, $c_{v}:$ Wärmekapazität, $T:$ Temperatur) nicht mehr gültig ist, muß eine allgemeinere Form der Zustandsgleichung Gl. (2.27) verwendet werden. Zur Berechnung der numerischen Flußfunktion werden unter anderen der Druck $p$, die Schallgeschwindigkeit $c_{s}(\rho, \epsilon)$ und der Adiabatenexponent $\gamma(\tau, \epsilon)$

$$
\begin{aligned}
c_{s}^{2} & :=-\tau^{2}\left(\frac{\partial p(\tau, \epsilon)}{\partial \tau}-p(\tau, \epsilon) \frac{\partial p(\tau, \epsilon)}{\partial \epsilon}\right), \\
\gamma(\tau, \epsilon) & :=\frac{c^{2}(\tau, \epsilon)}{\tau p(\tau, \epsilon)}
\end{aligned}
$$

$\left(\tau:=\frac{1}{\rho}\right)$

benötigt. Da sich pro Zeitschritt bei schon berechneten $\rho, \vec{v}, \vec{B}$ aus der Evolutionsgleichung der Gesamtenergie nur die thermische Energiedichte $\epsilon=\epsilon(\tau, p)$ berechnen läßt und nicht $p$ oder $T$, müssen diese thermodynamischen Größen anhand von $\epsilon$ berechnet werden. Dies läßt sich im Falle numerischer Simulationen auf unterschiedlichem Wege bewerkstelligen. Entweder man bestimmt mittels NewtonRaphson-Iteration ausgehend von einem Startwert in jedem Zeitschritt zur aktuellen thermischen Energiedichte $\epsilon$ die Temperatur oder den Druck und berechnet sich daraus $\gamma$ und $c_{s}$. Dies liefert zwar genaue Resultate, ist im Rahmen von zeitabhängigen Simulationsrechnungen allerdings erheblich rechenzeitintensiver als die Verwendung von tabellierten Zustandsgleichungen, die auf dieselbe Art und Weise allerdings vor der eigentlichen Simulationsrechnung erstellt werden müssen (Kap. 2.6). Für den approximativen Riemannlöser und den Strahlungstransportlöser bedeutet letzteres, daß in der Berechnung der Links- und Rechtseigenvektoren, der Eigenwerte der Jakobimatrix und des Strahlungsquellterms an den Stellen an denen die Schallgeschwindigkeit $c_{s}$, der Druck $p$, die Temperatur $T$, die innere Energie $\epsilon$ und der Adiabatenexponent $\gamma$ benötigt werden, sie aus tabellierten Zustandsgleichungen als Funktion schon bekannter thermodynamischer Größen entnommen werden. Dazu werden zwei tabellierte Zustandsgleichungen benötigt, die entweder $c_{s}^{2}(\rho, p)$, $\epsilon(\rho, p)$ und $T(\rho, p)$ oder $c_{s}^{2}(\rho, \epsilon), p(\rho, \epsilon)$ und $T(\rho, \epsilon)$ enthalten. Sie werden zu Beginn der Simulationsrechnung angelegt und können wie in Abb. 3.6 schematisch dargestellt, im Verlaufe der Rechnung korrigiert und verfeinert werden, insofern dies notwendig sein sollte. Bei jedem Zugriff auf die äquidistante Grundtabelle wird für ein Wertepaar $(a, b)$ [z.B. $(\rho, p)$ oder $(\rho, \epsilon)]$ der Funktionswert $f(a, b)$ aus den vier umliegenden Tabellenwerten $f\left(a_{22}, b_{22}\right), f\left(a_{23}, b_{23}\right), f\left(a_{32}, b_{32}\right), f\left(a_{33}, b_{33}\right)$ [Abb. 3.6 (links)] interpoliert und dann mit dem exakten Wert $f\left(a_{e x}, b_{e x}\right)$ an dieser Stelle verglichen. Gilt $t_{c}<\left|f(a, b)-f\left(a_{e x}, b_{e x}\right)\right|$, so wird an dieser Stelle eine feinere Tabelle gefordert, da die relative Abweichung zwischen interpoliertem und exak- 
tem Wert die vorgegebene Grenze $t_{c}$ überschreitet. Aus diesem Grunde wird unter jeder diese Zellen der Grundtabelle, in der nicht unter Einhaltung der Fehlergrenze interpoliert werden kann (Abb. 3.6 (links)) ein hierarchisch strukturierter $\mathrm{Ta}$ bellenbaum, bestehend aus $n$ äquidistant strukturierten Untertabellen eingerichtet. Dabei stellt die erste Untertabelle einer Makrozelle den ersten Verfeinerungslevels dar und ist in Abb. 3.6 (rechts) dargestellt. Jede weitere Untertabelle bedeutet eine Verfeinerung der nächst gröberen Untertabelle, beginnend bei der ersten unter einer Makrozelle eingerichteten Untertabelle. Diese $n$-fach hierarchische Tabellen-
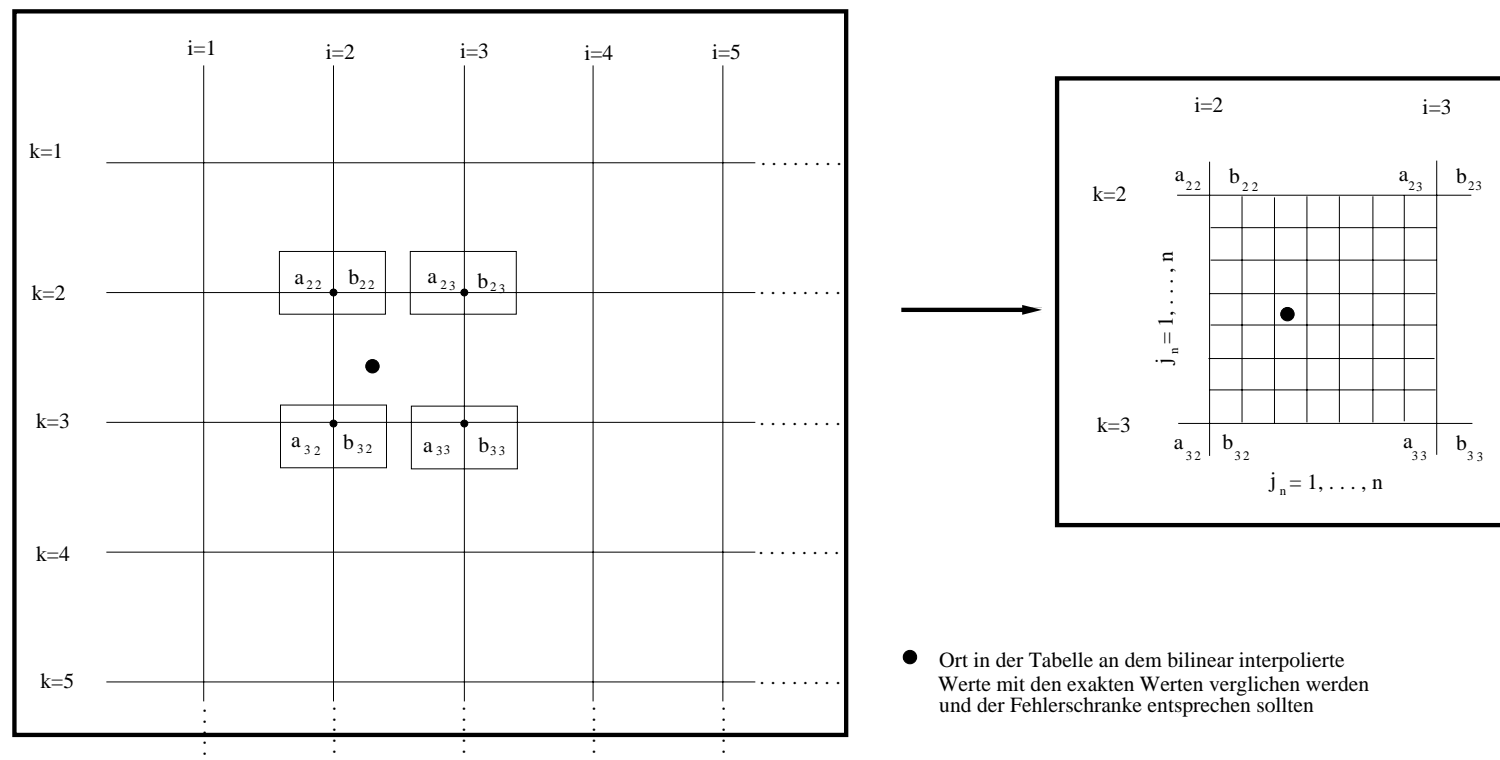

Abbildung 3.5: Unter jeder Zelle der äquidistant strukturierten Grundtabelle (links) in der $c_{s}^{2}(\rho, p), \epsilon(\rho, p)$ bzw. $T(\rho, p)$ oder aber $c_{s}^{2}(\rho, \epsilon), p(\rho, \epsilon)$ und $T(\rho, \epsilon)$ als Funktion von $(\rho, p)$ bzw. $(\rho, \epsilon)$ bilinear interpoliert werden sollen, wird ein Tabellenbaum bestehend aus $n$ ebenfalls äquidistant strukturierten Untertabellen eingerichtet, insofern in der Makrozelle nicht unter Einhaltung der relativen Fehlerschranke $t_{c}$ mit $t_{c}>\left|(a, b)-\left(a_{e x}, b_{e x}\right)\right|$ interpoliert werden kann. Dabei stellt die erste Untertabelle (rechts) eine Verfeinerung der Makrozelle dar und jede weitere Untertabelle (nicht mehr dargestellt) die Verfeinerung der nächst gröberen Untertabelle. Sobald für eine Makrozelle ein solcher Tabellenbaum eingerichtet wurde, wird nur noch in der feinsten Untertabelle interpoliert.

strukturierung kann für jede Zelle der Grundtabelle einmal durchgeführt werden, wobei der absolute maximale Verfeinerungslevel, oder die Gesamtzahl $n$ der Untertabellen, die einmal unter jeder Makrozelle eingerichtet werden können, von außen vorgegeben ist. Sobald einmal die Genauigkeit des Makrolevels nicht mehr ausgereicht hat, wird dieser Tabellenbaum unter der Makrozelle eingerichtet und danach nur noch in der Untertabelle, die die feinste Auflösung besitzt, interpoliert und niemals mehr im Laufe der Simulation im Makrolevel. Der Vergleich mit der exakten Lösung wird nur im Makrolevel für solche Makrozellen durchgeführt, für die noch kein solcher Tabellenbaum angelegt wurde. Mit dieser Strategie wird die Belegung von Speicherplatz minimiert, indem nur dort verfeinert wird, wo die ursprüngliche Tabellengenauigkeit nicht ausgereicht hat. In hier durchgeführten Anwendungen ist $t_{c}:=0.01$ gewählt worden. 
Der beschriebene approximative Riemannlöser des Godunovtyps wurde mit primitiven Variablen formuliert, was neben der einfacheren Berechnung der Eigenwerte und Eigenvektoren den weiteren Vorteil besitzt, daß die Gesamtzahl von Tabellenzugriffen pro Evolutionsschritt halbiert wird und somit eine erhebliche Rechenzeitersparnis nach sich zieht ([15]).

\subsection{Gitteradaption}

Die grundsätzliche Idee bei der Gitteradaption besteht in einer verfeinerten Gitterauflösung in jenen Bereichen eines unstrukturierten Dreiecksgitter in denen es aufgrund der Lösung notwendig ist. Auf diese Weise wird der Bedarf an Rechenzeit und Speicherplatz minimiert. Aufgrund der zu Beginn dieses Kapitels (Kap. 3) bereits skizzierten physikalischen Prozesse brauchen wir eine feinere Triangulierung im Bereich von Schocks, Kontaktunstetigkeiten und Verdünnungswellen, um diese möglichst genau aufzulösen. Da es sich dabei nicht um statische Phänomene handelt, reicht es nicht aus sehr fein aufgelöst zu rechnen, da dies dem Wünschen nach Rechenzeit- und Speicherplatzersparnis zu widerlaufen würde, sondern in Bereichen, in denen diese Auflösung nicht notwendig ist, gröber aufgelöst zu rechnen. Zur Adaption des Gitters wird ein lokales Verfeinern durch eine Halbierung der entsprechenden Gitterzelle bewirkt und das lokale Vergröbern durch das Aufheben einer durch Verfeinerung entstandenen Gitterstrukturierung. Als Adaptionskriterium zur Anpassung der Triangulierung an die numerische Lösung wird ein Schätzer verwendet, der den relativen Unterschied der Massendichte zwischen zwei Nachbardreiecken berücksichtigt. Der Schätzer im Dreieck $T_{j}$ ist:

$$
\eta_{i j}^{n}:=\left|\frac{\rho_{j}^{n}-\rho_{i}^{n}}{\rho_{j}^{n}}\right|
$$

$\left(\rho_{j}^{n}, \rho_{j}^{n}\right.$ : Massendichte in den Dreiecken $\left.T_{j}, T_{i}\right)$.

Um gegebenenfalls ein rasches Alternieren von Vergröberung und Verfeinerung zu verhindern werden unterschiedliche Verfeinerungs und Vergröberungsschranken verwendet:

$$
\begin{array}{llrl}
\eta_{j}^{n}>C_{\text {fein }} \longrightarrow & \text { verfeinere } & T_{j} \\
\eta_{j}^{n}<C_{\text {grob }} \longrightarrow & \text { vergröbere } & T_{j}
\end{array}
$$

$\left(C_{\text {fein }}>C_{\text {grob }}\right) . \mathrm{Im}$ aktuellen Fall sind $C_{\text {fein }}=0.1$ und $C_{\text {grob }}=0.05$ gewählt worden. 


\subsection{Zeitschrittweitensteuerung}

Um die Stabilität eines expliziten numerischen Verfahrens gewährleisten zu können müssen sogenannte Stabilitätsbedingungen an die Gitterzellengröße und die Zeitschrittweite $\Delta t_{n}$ gestellt werden. Mit einer vorgegeben Konstanten CFL $<1$, welche problemabhängig zu wählen ist, unterliegt die numerische Zeitschrittweite $\Delta t_{n}$, im Falle eines unstrukturierten Dreiecksgitters, folgender Bedingung:

$$
\Delta t_{n}:=\mathrm{CFL} \cdot \min _{T_{j} \in \mathcal{T}}\left\{\frac{\left|T_{j}\right|}{\max _{k=1, \ldots, 7}\left|\lambda_{k}\right| \cdot \max _{l=1,2,3}\left|S_{j l}\right|}\right\},
$$

wobei $\lambda_{k}, k=1, \ldots, 7$ die Eigenwerte der MHD-Gleichungen sind ([106]).

\subsection{Entdimensionierung des Gleichungssystems}

Das Gleichungssystem (2.46) wurde vor seiner numerischen Berechnung entdimensioniert. Dies wurde dergestalt durchgeführt, daß die im Gleichungssystem vorkommenden Variablen $\rho, T, p, \vec{v}, E, \vec{B}, L, t, \vec{g}$ als Produkte von einer dimensionsbehafteten Einheit und einer dimensionslosen Variablen dargestellt wurden: $\rho=\rho_{0} \cdot \tilde{\rho}$, $T=T_{0} \cdot \tilde{T}, p=p_{0} \cdot \tilde{p}$ etc. Die Einheiten sind wie nachfolgend dargestellt gewählt und erklären sich folgendermaßen: $\rho_{0}, T_{0}, p_{0}$ sind die thermodynamischen Variablen der Anfangslösung (Kap. 2.9.5) am Gebietsunterrand, $\overline{\mu_{b o t}}$ ist das dazugehörende mittlere Molekulargewicht. $L_{0}$ ergibt sich aus der Annahme, daß die vertikale Gesamtausdehnung des Rechengebiets $L=2 L_{0}$ beträgt. Die dimensiontragenden Einheiten betrugen in den beschriebenen Anwendungen:

$$
\begin{aligned}
& L_{0}=6.7245 \cdot 10^{7}[\mathrm{~cm}], \quad T_{0}=14620 .[\mathrm{K}] \text {, } \\
& \rho_{0}=2.537 \cdot 10^{-6}\left[\frac{\mathrm{g}}{\mathrm{cm}}\right] \text {, } \\
& \bar{\mu}_{\text {bot }}=1.057, \quad p_{0}=\frac{\rho_{0} R_{\text {gas }} T_{0}}{\bar{\mu}_{\text {bot }}}=2.917 \cdot 10^{6}\left[\frac{\text { dyn }}{\mathrm{cm}^{2}}\right] \text {, } \\
& g_{0}=\frac{L_{0}}{t_{0}^{2}}\left[\frac{\mathrm{cm}}{\mathrm{s}^{2}}\right], \quad v_{0}=\frac{L_{0}}{t_{0}}\left[\frac{\mathrm{cm}}{\mathrm{s}}\right], \\
& B_{0}=\sqrt{\rho_{0} \cdot v_{0}^{2}}\left[\frac{g}{s^{2} c m}{ }^{\frac{1}{2}}\right], \quad t_{0}=\sqrt{\frac{L_{0}^{2} \bar{\mu}_{\text {bot }}}{R_{g a s} T_{0}}}[s] .
\end{aligned}
$$




\section{Kapitel 4}

\section{Strahlungstransport}

\subsection{Strahlungsstransport(RT)-Physikalische Annah- men}

Die Bestimmung der spezifischen Intensität (Gl. 2.40) mittels der RT-Gleichung in ihrer stationären Form

$$
\vec{\mu} \cdot \nabla I_{\nu}=\kappa_{\nu} \rho\left(S_{\nu}-I_{\nu}\right)
$$

erfordert die Kenntnis des Absorptionskoeffizienten $\kappa_{\nu}$, der Massendichte $\rho$ und der Quellfunktion $S_{\nu}$. Da die totale Absorption in der solaren Photosphäre hundertausende von verschiedenen Spektrallinien umfaßt, sind etwa $10^{6}-10^{7}$ Frequenzpunkte notwendig, um die genaue Frequenzabhängigkeit zu berücksichtigen. Im Falle von 2D oder 3D Simulationen ist dies zu rechenzeitintensiv. Daher nutzt man die vereinfachende Annahme der grauen Näherung ([92]). Dabei werden alle frequenzabhängigen Variablen, die zur Lösung der Gl. (4.1) notwendig sind als frequenzunabhängig betrachtet und durch ihre frequenzintegrierten Pendants ersetzt:

$$
I=\int_{0}^{\infty} I_{\nu} d \nu \quad, \quad J=\int_{0}^{\infty} J_{\nu} d \nu \quad, \quad F=\int_{0}^{\infty} F_{\nu} d \nu
$$

Die frequenzintegrierte Planckfunktion wird dabei durch das Stefan-BoltzmannGesetz beschrieben:

$$
B(T)=\int_{0}^{\infty} B_{\nu}(T) d \nu=\frac{\sigma_{r a d}}{4 \pi} T^{4}
$$

$\left(\sigma_{\text {rad }}=7.565 \cdot 10^{-15}\left[\mathrm{erg} / \mathrm{cm}^{3} / \mathrm{K}^{4}\right]:\right.$ Strahlungskonstante $)$.

Auch der Absorptionskoeffizient ist in der grauen Näherung frequenzunabhängig 
und wird durch eine sinnvolle Mittelung aller fequenzabhängigen $\kappa_{\nu}$ ermittelt. Im aktuellen Fall wurde $\bar{\kappa}$ durch die Rosselandsche Mittelungsvorschrift ([48]) bestimmt:

$$
\frac{1}{\bar{\kappa}}=\frac{\int_{0}^{\infty} \frac{1}{\kappa_{\nu}} \frac{d B_{\nu}(T)}{d T} d \nu}{\int_{0}^{\infty} \frac{d B_{\nu}(T)}{d T} d \nu} .
$$

Dieses gewichtet solche Spektralbereiche, in denen die Opazität geringer ist und somit mehr Energie transportiert wird, besonders und garantiert, daß der, unter seiner Verwendung, berechnete frequenzintegrierte Srahlungsstrom gleich dem beobachteten solaren Strahlungsstrom $\vec{F}_{\odot}^{r a d}$ ist:

$$
\int_{\nu} \vec{F}_{\nu}^{r a d} d \nu=\vec{F}_{\odot}^{r a d}
$$

Eine weitere wichtige Vereinfachung stellt die Annahme des Lokalen Thermodynamischen Gleichgewichts (LTE) dar. Dies besagt, daß sich die Besetzungsdichten der atomaren Niveaus und der Ionisationszustände lokal im thermodynamischen Gleichgewicht befinden und dementsprechend mit der Boltzmann- und der Sahagleichung berechnet werden können und ist dann gerechtfertigt, wenn die mittlere freie Weglänge eines Photons viel kleiner ist als die Wegstrecke über die sich die Temperatur des Gases signifikant ändert. Als Konsequenz daraus kann die frequenzabhängige Quellfunktion gleich der Planck-Funktion gesetzt werden:

$$
S_{\nu}:=B_{\nu}(T)=\frac{2 h \nu^{3}}{c^{2}} \frac{1}{\exp \left(\frac{h \nu}{k T}\right)-1}
$$

( $\nu$ : Frequenz, $k$ : Boltzmann-Konstante, $T$ : Temperatur, $h:$ Planck-Konstante, $c$ : Lichtgeschwindigkeit).

Obwohl in den oberen Bereichen der Photosphäre ab

$$
\tau_{5000}=\int_{0}^{z^{\prime}} \kappa_{5000}(z) \rho(z) d z \leq 10^{-2}
$$

eine deutliche Abweichung vom LTE zu verzeichnen ist, ist diese Annahme für die verbleibenden atmosphärischen Bereiche gut ([56]), weil dort die Stöße mit Elektronen, die eine thermische Geschwindigkeitsverteilung einnehmen, mit zunehmender Tiefe zunehmenden Eifluß auf die Besetzungszahlen gewinnen $\left(\tau_{5000}\right.$ : optische Tiefe bei $5000 \AA ̊$ ). 


\subsection{RT-Löser}

Die Lösung der stationären RT-Gleichung Gl. (4.1) im Rahmen (magneto)hydrodynamischer Simulationen stellt nun einige Bedingungen:

- Da der aktuelle Fall von 2D-Simulationen eine Vorstufe zu 3D-MHD-Simulationen ist, muss der RT-Löser unabhängig von der verwendeten Gittergeometrie (z.B. Dreiecke, Tetraeder, Vierecke, Quader) und der Gitterstruktur sein und sich ohne größeren Aufwand auf 3D erweitern lassen.

- Da die Berechnung auf Parallelrechnern, welche mit verteilten Speichern arbeiten, durchgeführt werden soll, muß der RT-Löser lokal arbeiten. Deshalb soll die Kommunikation zwischen den Prozessoren, die zu längeren Berechnungszeiten führt, reduziert werden.

- Da moderne (M)HD-Gleichungssystemlöser wenigstens von 2. Genauigkeitsordnung sind, soll der RT-Löser von der gleichen Konvergenzordnung sein.

- Da die Berechnung des Strahlungsquellterms (Kap. 2.7) sehr aufwendig ist im Vergleich zur Berechnung der magnetohydrodynamischen Variablen, muß der RT-Löser sehr schnell arbeiten.

- Der RT-Löser muß sowohl große Temperatur- und Dichtegradienten, als auch starke Variationen in $\kappa$ handhaben können (teilweise $\kappa \propto T^{10}$ im Bereich photosphärischer Temperaturen).

Im Hinblick auf diesen Anforderungskatalog untersuchten wir verschiedene Methoden zur Berechnung der Strahlungstransportgleichung. Auf der einen Seite wurde das Discontinuous-Galerkin-Verfahren ([52]), welches ein Finite-Elemente-Verfahren ist, von A. Dedner am Institut für Angewandte Mathematik der Universität Freiburg (IAM) zur Lösung der Strahlungstransportgleichung getestet und mir zur Verfügung gestellt. Da seine Eigenschaften sowohl vom mathematischen, als auch vom numerischen Standpunkt gut verstanden sind ([41], [12]), diente es im weiteren als Referenzverfahren. Auf der anderen Seite sind zwei Methoden, die auf Charakteristiken basieren, untersucht worden. Dabei handelt es sich um die longcharacteristics-Methode, welche von Mihalas et al. (1978) ([57]) vorgeschlagen wurde und die short-charcteristics-Methode, die von Kunasz und Auer (1988) ([47]) entwickelt und von Bruls et al. (1999) ([4]) auf unstrukturierte Dreiecksgitter übertragen worden ist. Da diese Methoden wie nachfolgend dargestellt (Kap. 4.3.1) entweder zu langsam und ungenau sind oder ineffizient auf Parallelrechnern mit verteilten Speichern arbeiteten, wurde eine neue Methode, die ExtendedShort-Characteristics-Methode (ESC) von A. Dedner (IAM) und mir entwickelt ([14]). Diese kombiniert den Finite-Elemente-Ansatz mit lokalen Ansatzfunktionen und den short-characteristics-Ansatz und wird anhand mehrerer Testprobleme mit den oben erwähnten Methoden verglichen. Von mir wurde in dieser Zusammenarbeit anstelle des klassischen short-characteristics-Lösers ein anderer short- 
characteristics-Löser entwickelt und getestet. A.Dedner trug die Idee der FinitenElemente bei und lieferte den Alogrithmus zur Bestimmung der Abarbeitungsreihenfolge. Im Zusammenspiel des short-characteristics-Lösers und des FinitenElemente-Ansatz lassen sich damit ESC-Methoden beliebiger Konvergenzordnungen herleiten. Weiterhin wurde von mir die ESC-Methode auf die Testprobleme angewendet und somit versucht, die charakteristischen Eigenschaften der neuentwickelten ESC-Methode zu quantifizieren.

\subsubsection{Extended-Short-Characteristics (ESC)-Methode}

Die Idee hinter der ESC-Methode ist die Kombination des short-characteristicsAnsatzes und des Finiten-Elemente-Ansatzes. Dadurch läßt sich eine ganze Klasse von neuen Methoden herleiten, die sich in ihrer Konvergenzordnung und/oder durch die geometrische Struktur der numerischen Gitterzellen (Dreicke, Vierecke, Hexaeder, Tetraeder, Quader etc.), die zur Berechnung der Gl. (4.1) zugrunde gelegt werden, unterscheiden. Zur formalen Beschreibung der ESC-Methode betrachten und studieren wir diese Gleichung auf einem begrenzten, offenen Teilgebiet $\omega$ des $\mathbb{R}^{n}$ mit $n \geq 2$, das entweder das gesamte Rechengebiet $\Omega$ oder ein Teilgebiet von $\Omega$, z.B. ein einzelnes Gitterelement sein kann. Auf dem Teilgebiet $\omega$ wird eine genäherte Lösung $\bar{I}$ der Strahlungstransportgleichung für eine Strahlungsrichtung $\vec{\mu} \in \mathbb{R}^{n}$ gesucht:

$$
\begin{array}{rlrl}
\vec{\mu} \cdot \nabla I+\chi I & =\chi S & & \text { in } \omega \\
I & =g & & \text { auf dem Gebietsrand } \\
& \partial \omega_{-}
\end{array}
$$

(mit $\chi=\kappa \rho)$.

$g \in C^{0}\left(\partial \omega_{-}\right)$ist dabei die Intensität auf der Einflußseite, d.h. auf der Seite, auf welcher Strahlen in Richtung $\vec{\mu}$ in das Gebiet eintreten

$$
\partial \omega_{-}^{\vec{\mu}}=\partial \omega_{-}:=\left\{\vec{x} \in \partial \omega_{-} \mid \vec{\mu} \cdot \vec{n}(\vec{x})<0\right\} \quad,
$$

wobei $\vec{n}(\vec{x})$ die Oberflächennormale der Einflußkante $\partial \omega$ am Punkte $\vec{x} \in \partial \omega$ ist und $\chi, S \in C^{0}(\bar{\omega})$ die auf dem Element bekannten Daten sind. Das Ziel ist nun die Ermittelung einer approximativen Lösung $\bar{I}$ der exakten Lösung $I$ der Gl. (4.8) in einem gegebenen Funktionenraum $P(\omega)$ mit der endlichen Dimension $r$. Falls $\omega$ z.B. ein Element eines Dreiecksgitters ist, stellt $P(\omega)$ den Polynomraum auf diesem Dreieckselement dar. Für einen Satz von Basisfunktionen $\left\{\varphi_{i}\right\}_{1 \leq i \leq r}$ des $P(\omega)$ und Punkten $\vec{p}_{i} \in \bar{\omega}(1 \leq i \leq r)$ wird dabei folgendes angenommen:

$$
\varphi_{i}\left(\vec{p}_{j}\right)=\delta_{i j} \quad \text { mit } \quad 1 \leq i, j \leq r \quad .
$$

Ferner muß ein $m \geq 0$ existieren mit:

$$
\begin{array}{cll}
\vec{p}_{i} \in \partial \omega_{-} & \text {für } \quad 1 \leq i \leq m, \\
\vec{p}_{i} \in \omega \backslash \partial \omega_{-} & \text {für } \quad m<i \leq r .
\end{array}
$$


Diese Formale Darstellung der ESC-Methode wird durch Abb. 4.1 anschaulich gemacht. Nun läßt sich die approximative Lösung $\bar{I}$ in der Basis $\left\{\varphi_{i}\right\}_{1 \leq i \leq r}$ darstellen

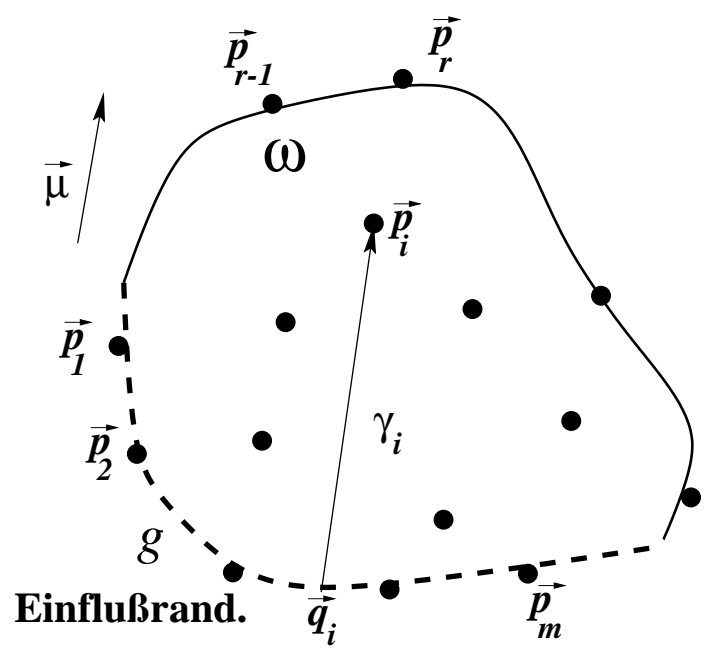

Abbildung 4.1: Skizzierung der ESC-Methode. Der Einflußrand g, mit den auf ihr liegenden Punkten [Gl. (4.10)] $\vec{p}_{1} \ldots \vec{p}_{m}$, ist gestrichelt dargestellt. $\vec{q}_{i}$ stellt den Anfangspunkt der short-characteristic $\gamma_{i}$, der auf der Einflußkante liegt, dar. Die short-characteristic $\gamma_{i}$ verbindet diesen Randpunkt $\vec{q}_{i}$ mit einem der Punkte $\vec{p}_{i} \ldots \vec{p}_{r}$, welche in einem Element liegen. An diesen Punkten, welche durch Gl. (4.11) beschriebenen werden, nehmen die Basisfunktionen Gl. (4.9) den Wert 1 an.

und hat folgende Form:

$$
\bar{I}(\vec{x})=\sum_{j=1}^{r} I_{j} \varphi_{j}(\vec{x})
$$

Die unbekannten Koeffizienten $I_{j}$ müssen nun an den Punkten ermittelt werden, an denen die Bedingung Gl. (4.9) erfüllt ist, die Basisfunktionen also den Wert 1 annehmen. Da die Intensität auf der gesamten Einflußseite als bekannt betrachtet wird, sind damit auch die Koeffizienten $I_{j}$ für $1 \leq j \leq m$ bekannt:

$$
I_{j}=g\left(\vec{p}_{j}\right)
$$

Um nun die anderen Koeffizienten berechnen zu können, nutzen wir die Methode der short-characteristics. Eine solche short-characteristic (kurze Charakteristik), dargestellt durch

$$
\gamma_{j}:=\left\{\vec{x} \mid \vec{x}=\vec{q}_{j}+s \vec{\mu}, 0 \leq s \leq s_{j}\right\} \subset \omega
$$

$\left(s_{j}:=\left|\vec{q}_{j}-\vec{p}_{i}\right|\right)$

beschreibt nun die kürzeste Verbindung des Punktes

$$
\vec{p}_{i} \in \omega \backslash \partial \omega_{-} \quad \text { für } \quad m<i \leq r .
$$


mit dem Schnittpunkt auf der Einflußseite. Entlang dieser short-characteristic $\gamma_{j}$ (Abb. 4.1) erfüllt die Intensität $v_{j}(s):=I\left(\vec{q}_{j}+s \vec{\mu}\right)$ folgendes Anfangswertproblem:

$$
\begin{aligned}
v_{j}^{\prime}(s)+\chi\left(\vec{q}_{j}+s \vec{\mu}\right) v_{j}(s) & =\chi\left(\vec{q}_{j}+s \vec{\mu}\right) S\left(\vec{q}_{j}+s \vec{\mu}\right) \quad \text { for } \quad 0 \leq s \leq s_{j} \\
v_{j}(0) & =g\left(\vec{q}_{j}\right) .
\end{aligned}
$$

Mit $I_{j}:=v_{j}\left(s_{j}\right)$ für $m<j \leq r$ lassen sich nun die unbekannten Koeffizienten $I_{j}$ berechnen. Zusammenfassend wird nun die approximative Lösung der Strahlungstransportgleichung Gl. (4.1) beschrieben durch Gln. (4.12), (4.13) und (4.14)

\subsubsection{Realisation auf unstrukturierten Gittern}

Im folgenden wird die Umsetzung der ESC-Methode für zwei Raumdimensionen beschrieben. Ein möglicher Weg, um eine approximative Lösung des Problems :

$$
\begin{aligned}
& \vec{\mu} \cdot \nabla I+\chi I=\chi S \text { in } \Omega \text {, } \\
& I=g \quad \text { in } \partial \Omega_{-} \text {, }
\end{aligned}
$$

für eine gegebene Strahlrichtung $\vec{\mu}$ zu finden, ist die Methode der long-characteristics, welche von Mihalas et al. 1978 ([57]) für kartesische Gitter vorgeschlagen wurde. Dabei setzt man $\omega:=\Omega$, d.h. dem gesamten Rechengebiet gleich, und die Punkte $\vec{p}_{i}$ Gl. (4.11) sind die Knoten des Gitters $\mathbf{T}_{h}$. Das Anfangswertproblem Gl.(4.14) muß nun für jede einzelne Characteristik, die einen Knoten mit ihrem entsprechenden Startpunkt auf der Einflußseite verbindet, gelöst werden (Abb. 4.2).

Dieser Ansatz wurde allerdings verworfen, da zur Berechnung der Intensität an einem solchen Punkt $\vec{p}_{i}$ die Informationen aller Gebietselemente, durch die die Charakteristik 'läuft', notwendig sind. Dadurch wird das Schema nichtlokal und die Portierung des Rechenprogramms auf Parallelrechner, die mit verteiltem Speicher arbeiten, aufgrund der erforderlichen Prozessorkommunikation, sehr ineffizient. Dies ist dadurch begründet, daß das numerische Gitter partitioniert wird, d.h. die Gitterelemente auf die einzelnen Prozessoren aufgeteilt und von diesen 'abgearbeitet' werden. Je nach Aufteilung kann es dazu kommen, daß einzelne Prozessoren auf andere Prozessoren warten müssen, weil benachbarte Gebietselemente unterschiedlichen Partitionen zugeteilt worden sein können und somit die Intensität eines Elements, von einem Prozessor noch nicht berechnet werden kann, da die dazu notwendige Einflußrandintensität, als Intensität eines anderen Elements von von einem anderen Prozessor berechnet wird. Weiterhin erfordert die Lösung der Gl.(4.15) entlang solch langer Charakteristiken, zeitaufwendige Schnittpunktsbestimmungen der einzelnen Charakteristiken mit den Seiten der Elemente, die sie schneiden. Der von uns beschrittene und nachfolgend beschriebene Weg enthält die Umsetzung der ESC-Methode auf jedem einzelnen Gitterelement und untergliedert sich in drei aufeinanderfolgende Einzelschritte.

\section{Bestimmung einer Abarbeitungsreihenfolge einzelner Gitterelemente ( $\omega$ - $=T)$ :}




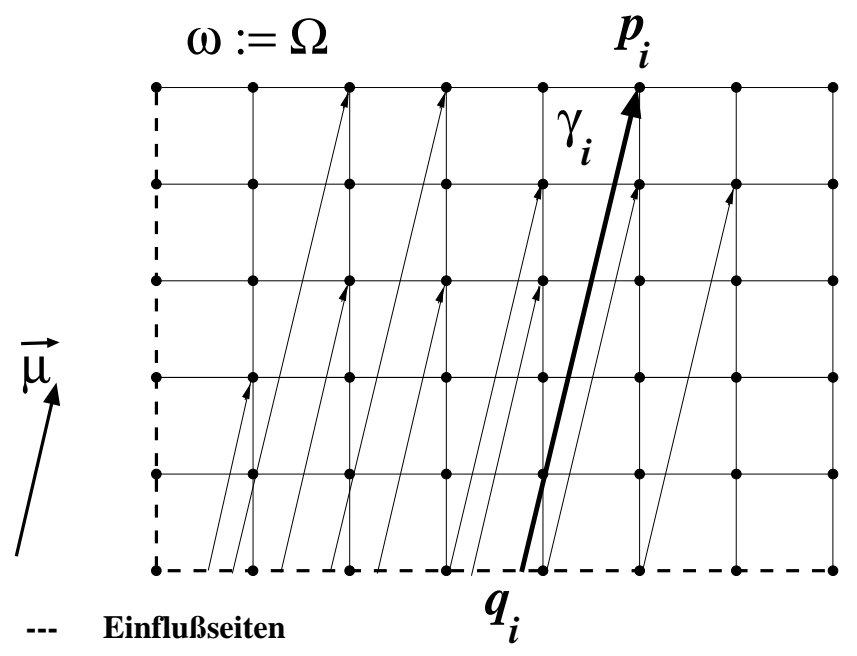

Abbildung 4.2: Long-characteristics Methode $(\omega=\Omega)$ : Die Strahlungstransportgleichung wird entlang der Charakteristiken $\gamma_{i}$, die Punkte $p_{i}$ Gl. (4.11) mit ihren Startpunkten $q_{i}$ $(i=1,2 \cdots n)$ auf der Einflußseite verbinden, gelöst.

Die short-characteristics-Methode kann ohne weiteres zur Ermittelung von $I_{T}$ für ein einzelnes Element $T$ der gegebenen Triangulierung (Gesamtgitter) angewandt werden, sofern die Intensität auf der Einflußseite bekannt ist. Falls die Einflußseite mit der Ausflußseite eines anderen Elementes zusammenfällt, muß demnach erst $I_{T}$ dieses Elements berechnet werden. Aus diesem Grunde ist eine exakte Abarbeitungsreihenfolge der Elemente notwendig. Die Bestimmung ist im Falle eines, mit unstrukturiertem, adaptivem Lösungsgitter, auf Parallelrechnern arbeitenden (M)HD-Lösers kompliziert. Ein solcher Algorithmus ist von A. Dedner (IAM) ([12], [14]) entwickelt und mir zur Verfügung gestellt worden.

\section{Lösung von Gl. (4.15) auf einem einzelnen Gitterelement:}

Wenn die Reihenfolge, in der die einzelnen Elemente abgearbeitet werden bekannt ist, folgt die Lösung von Gl. (4.15) auf einem einzelnen Element $T$. Dazu müssen die Punkte $\vec{p}_{i} \in \mathbf{T}_{h}(i=1,2, \cdots, n)$ und die Basisfunktionen $\left\{\varphi_{i}\right\}_{1 \leq i \leq r}$ des Polynomraums $P(T)$ mit $\varphi_{i}\left(\vec{p}_{j}\right)=\delta_{i j} \quad($ mit $1 \leq i, j \leq r)$ bestimmt werden. Wir betrachten im Folgenden zwei Möglichkeiten, die als ESC1 und ESC2 bezeichnet werden und sich in den Basisfunktionen und den Positionen der $\vec{p}_{i}$ unterscheiden.

ESC1: Methode erster Ordnung, mit linearen Basisfunktionen. Die drei Punkte $\vec{p}_{1}, \vec{p}_{2}, \vec{p}_{3} \in T$, an denen die Koeffizienten $I_{j}$ durch Lösung des Anfangswertproblems (4.14) bestimmt werden, sind die drei Knoten (Eckpunkte) des Dreiecks an. Die entsprechenden Basisfunktionen sind in Abb. 4.3 (links) abgebildet.

ESC2: Methode zweiter Ordnung mit quadratischen Basisfunktionen. In diesem Fall müssen an sechs definierten Punkten $p_{i}$ die Koeffizienten $I_{j}$ von Gl. (4.13) berechnet werden. Diese sind die 3 Knoten (Eckpunkte) und die 3 Kantenmittelpunkte. Die entsprechenden Basisfunktionen sind in Abb. 4.3 (Mitte und rechts) abgebildet. 


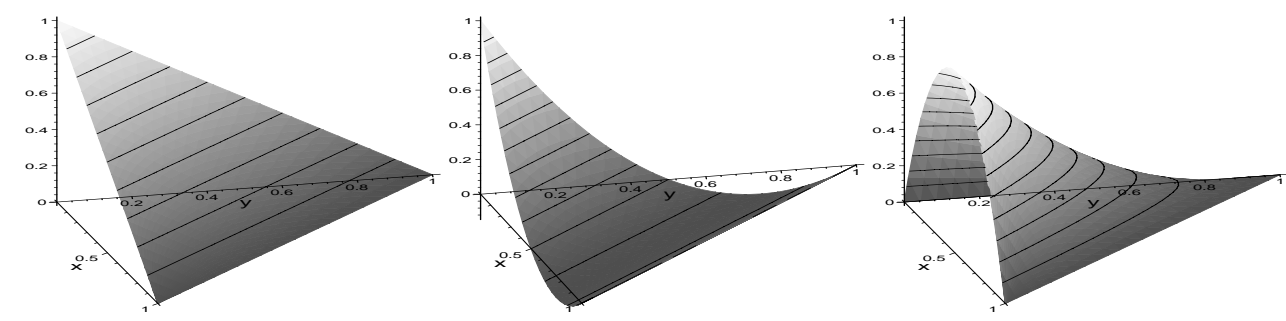

Abbildung 4.3: Knoten-Basisfunktionen für die ESC1-Methode (links) und die ESC2Methode (Mitte) und die Kantenmittelpunkts-Basisfunktionen für die ESC2-Methode.

\section{Lösung des short-characteristics Problem:}

Die Berechnung der Koeffizienten $I_{j}$ erfolgt mittels der short-characteristicsMethode ([47]). Dabei wird die gewöhnliche Differentialgleichung Gl. (4.14) entlang einer Charakteristik, die einen Punkt $\vec{q}_{i}$ auf der Einflußseite mit dem dazu korrespondierenden Punkt $\vec{p}_{i}$ (an dem der Koeffizient berechnet werden soll), verbindet, gelöst.

Im Fall der ESC1-Methode müssen zwei Möglichkeiten unterschieden werden. Entweder ein Dreieck $T$ besitzt zwei Einflußseiten und die Koeffizienten $I_{i}$ an den drei Knoten sind somit schon bekannt und die lineare Funktion $I_{T}$ bestimmt, oder aber von drei Koeffizienten liegen nur zwei auf einer Einflußseite und der dritte Koeffizient muß berechnet werden. Im zweiten Fall verbleibt die Berechnung des Koeffizienten an dem Punkt, der der Einflußseite gegenüber liegt. Diese Konstellation ist in Abb. 4.5 (links) für ein Element dargestellt. Die Intensität an Punkt C muß berechnet werden, wobei die Intensität $I_{\mathrm{cc}}$ am Startpunkt der Charakteristik cc durch die Lösung am Nachbardreieck bereits gegeben ist. Für ein numerisches Gitter bestehend aus Dreieckselementen ist diese Vorgehensweise in Abb. 4.2.2 im Falle der ESC1-Methode für eine Richtung $\vec{\mu}$ skizziert. Im Fall der ESC2-Methode müssen zwei Fälle, die in Abb. 4.5 (Mitte) und Abb. 4.5 (rechts) dargestellt sind, betrachtet werden. Im ersten Fall mit zwei Einflußseiten (Abb. 4.5 Mitte) muß die Intensität am Kantenmittelpunkt F der Ausflußseite berechnet werden. Die Startintensität der Charakteristik am Punkt ff ist ebenfalls durch die Lösung am schon abgehandelten Nachbardreieck gegeben. Im zweiten Fall (eine Einflußseite, Abb. 4.5 rechts) sind die Koeffizienten $I_{\mathrm{ee}}, I_{\mathrm{cc}}$ und $I_{\mathrm{dd}}$ an den Punkten ee, cc und dd bekannt und die Intensitäten an den Punkten E, C und D müssen berechnet werden. Zur Lösung der gewöhnlichen Differentialgleichung Gl. (4.14) wurden mehrere Methoden untersucht. Zwei davon, im weiteren als KA1 und KA2 bezeichnet, verwenden die formale Lösung:

$$
v_{i}(s)=v_{i}(0) e^{-\Delta \tau(0, s)}+\int_{0}^{s} S(\sigma) e^{-\Delta \tau(\sigma, s)} \mathrm{d} \sigma
$$

mit

$$
\Delta \tau(a, b):=\int_{a}^{b} \kappa(\sigma) \rho(\sigma) \mathrm{d} \sigma
$$




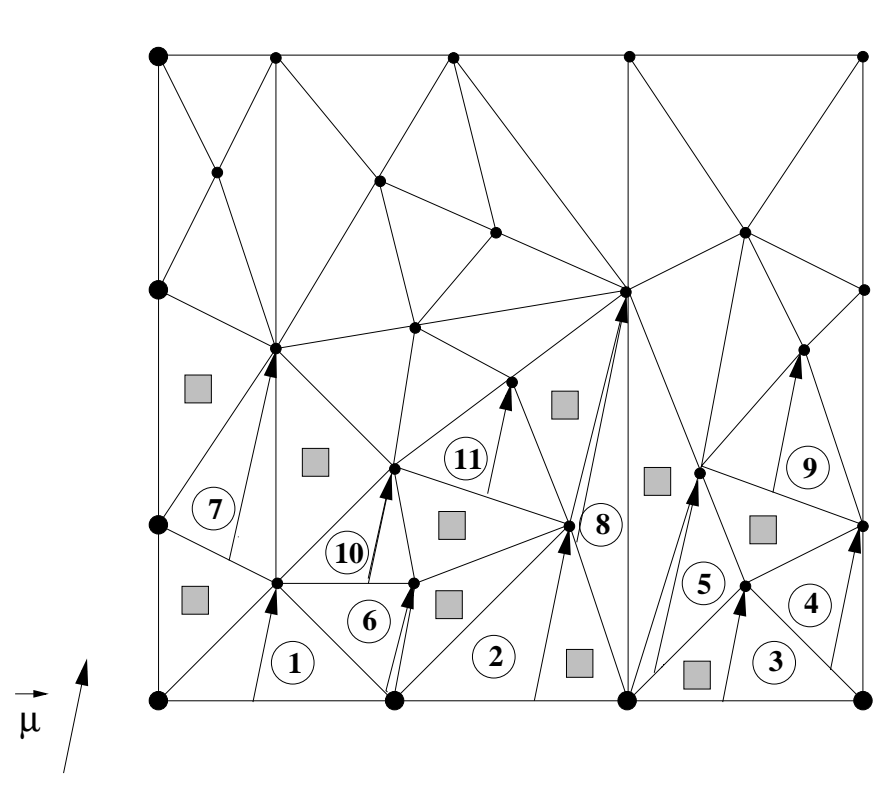

- : Einflußrandknoten , an denen Intensität gegeben ist

- : Gebietknoten an denen Intensität berechnet werden muß

Nr) : Ziffer beschreibt Abarbeitungsreihenfolge

: durch Nachbarelemente vollständig bestimmte Dreiecke

$\vec{\mu}$ : Strahlenrichtung

1 : " short-characteristic ", transportgl. zu lösen ist

Abbildung 4.4: Schematische Darstellung des Ablaufs zur Lösung der Strahlungstransportgleichung auf einem Dreiecksgitter im Falle der ESC1-Methode für die Strahlenrichtung $\vec{\mu}$.
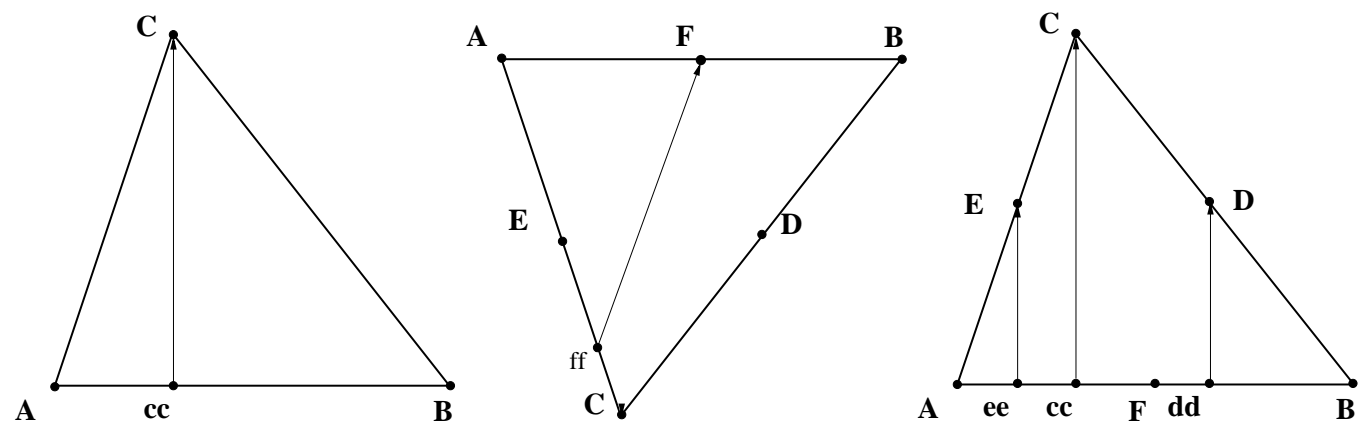

Abbildung 4.5: Um die Strahlungstransportgleichung auf einem einzelnen Element zu lösen, ist es notwendig die Intensität an verschiedenen Punkten zu berechnen, welche abhängig von der betrachteten Ordnung sind. Links: Im Fall der ESC1-Methode für Dreiecke mit nur einer Einflußseite muß die Intensität am Punkt $C$ entlang einer Charakteristik, deren Startintensität am Punkt cc bekannt ist, berechnet werden. Mitte and Rechts: In der ESC2-Methode müssen die Intensitäten abhängig von der Strahlenrichtung $\vec{\mu}$ entweder am Punkt F (zwei Einflußseiten, $\overline{A C}$ und $\overline{B C}$ ), oder an den Punkten E, $C, D$ (eine Einflußseite, $\overline{A B}$ ) mit den entsprechenden Startintensitäten am Punkt ff bzw. an den Punkten ee, cc und dd berechnet werden.

Die beiden anderen Lösungsverfahren (IRK, DRK) sind implizite bzw. diagonal implizite Runge-Kutta-Standardlöser für gewöhnliche Differentialgleichungen ([95]) und lassen sich ohne größeren Aufwand implementieren, da Gl. (4.14) eine lineare Differentialgleichung ist und somit nur ein lineares $2 \times 2-$ Gleichungssystem zu lösen ist. Da mit dem impliziten Runge-Kutta- 
Standardlöser die besten Resultate erzielt wurden und dieser in den zeitabhängigen magnetohydrodynamischen Simulationsrechnungen eingesetzt wird, wird im Anhang 8.1 eine ausführliche Beschreibung gegeben. Weitere betrachtete Lösungsmethoden basierten entweder auf numerischen Quadraturen zur Lösung des Integrals in Gl. (4.16) (Gauß-Legendre, Romberg) oder waren explizite Löser gewöhnlicher Differentialgleichungen (explizites Runge-Kuttaund explizites Euler-Verfahren). Sie führten zu erheblichen Schwierigkeiten bei einer starken Variation von $\kappa \rho$ im Rechengebiet $10^{-3}-10^{4}$, welche von der signifikanten Temperaturabhängigkeit von $\kappa\left(\propto T^{10}\right)$ herrührt, und wurden daher nicht weiter berücksichtigt. Die vier untersuchten Löser sind folgendermaßen charakterisiert:

- KA1: $\kappa \rho$, S werden durch lineare Funktionen approximiert, was zu einem analytischen Ausdruck für das Integral in Gl. (4.16) führt ([47], [4]).

- KA2: $\kappa \rho, \mathbf{S}$ werden durch Polynome zweiter Ordnung approximiert, was ebenfalls zu einem analytischen Ausdruck für das Integral in Gl. (4.16) führt ([47], [4]).

- IRK: Gl. (4.14) wird mit implizitem 2-Punkt-Runge-Kutta-Schema gelöst, wobei die besten Resultate mit der RADAU IIA Methode erzielt wurden ([95]).

- DRK: Gl. (4.14) wird mit einem einfach diagonal implizitem 2-PunktRunge-Kutta-Schema gelöst ([95]).

\subsubsection{Oszillationskorrektur}

Die Methoden 2. Ordnung zeigen oft ein oszillatorisches Verhalten, welches sogar zu unphysikalischen negativen Intensitäten führen kann. Dieses Verhalten resultiert aus der quadratischen Interpolation zwischen den Einflußrandintensitäten dreier Punkte. In Abb. 4.6 ist dies für den Fall eines Einflußrandes (vergl. Abb. 4.5 rechts) dargestellt. Die Intensität an den Punkten A, F und B ist als Einflußrandintensität bekannt. Zwischen ihnen sollen die Intensitäten an den Punkten ee, cc und dd quadratisch interpoliert werden. Dadurch können sich neue Extrema ausbilden und zu falschen Intensitätswerten auf dem Dreieck führen. Durch leichte Modifikationen der Einflußintensitäten $g$, dargestellt in Abb. 4.6, lassen sich diese Schwierigkeiten allerdings beheben:

\subsubsection{Periodische Randbedingungen}

Der bisher beschriebene Algorithmus kann auf jedes Gebiet $\Omega$ angewandt werden, sofern die Einflußseitenintensität bekannt ist. Im aktuellen Fall wurde entsprechend 


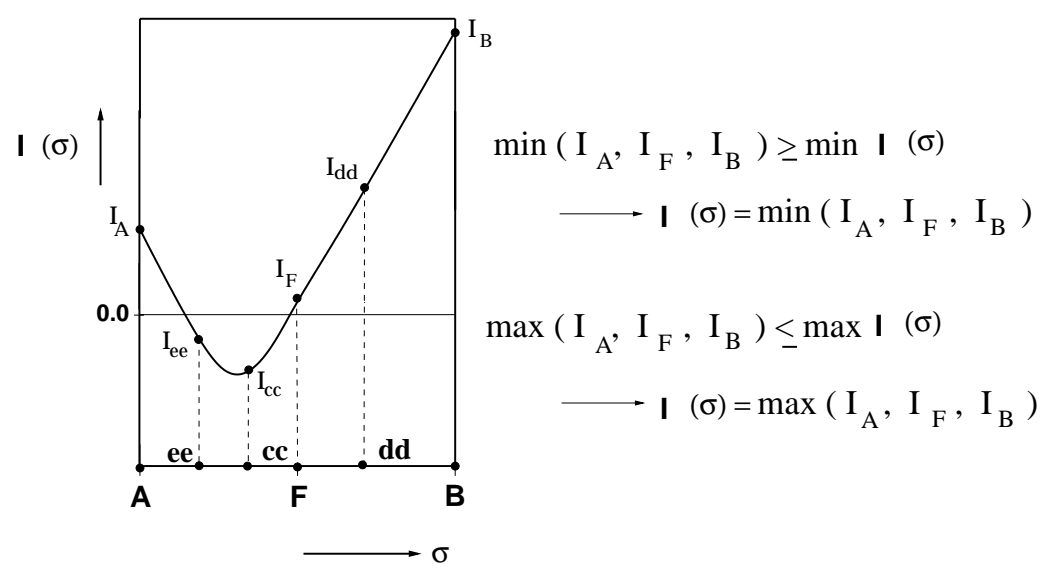

Abbildung 4.6: Quadratische Interpolation kann neue Extrema erzeugen, die zu falschen, ggf. sogar negativen, Intensitäten führen können. Dieses oszillatorische Verhalten mit daraus resultierenden falschen Einflußintensitäten $g$ läßt sich folgendermaßen beheben. Für den Fall, daß das Minimum der interpolierten Intensitäten $I_{\mathrm{ee}}, I_{\mathrm{cc}}$ und $I_{\mathrm{dd}}$ kleiner als das Minimum der Intensitäten an den Punkten A, F und B ist, so wird die interpolierte Intensität gleich dem kleinsten der Werte $I_{\mathrm{A}}, I_{\mathrm{F}}$ und $I_{\mathrm{B}}$ gesetzt. Für den Fall, daß das Maximum der interpolierten Intensitäten $I_{\mathrm{ee}}, I_{\mathrm{cc}}$ und $I_{\mathrm{dd}}$ größer als das Maximum der Intensitäten an den Punkten A, F und B ist, so wird die interpolierte Intensität gleich dem größten der Werte $I_{\mathrm{A}}, I_{\mathrm{F}}$ und $I_{\mathrm{B}}$ gesetzt.

der Strahlungsrichtung die Intensität vorgeschrieben. Für einen nach oben gerichteten Strahl $\left(\mu_{z}>0\right)$ wurde die Einflußintensität am unteren Rand mit der frequenzintegrierten Planck-Funktion Gl. (4.6) vorgeschrieben. Im Falle eines nach unten gerichteten Strahls $\left(\mu_{z}<0\right)$ findet keine Einstrahlung am oberen Gebietsrand statt $\left[I_{j}=g\left(\vec{p}_{j}\right)=0\right.$, Gl. (4.13)]. In beiden Fällen werden an den seitlichen Rändern periodische Randbedingungen angenommen und die Strahlungstransportgleichung wird für das gesamte Gebiet iterativ gelöst. Dabei wurde entsprechend einer der Strahlungsrichtungen $\vec{\mu}$, die in einem Iterationsschritt am Ausflußrand berechnete Intensität im nächsten Iterationsschritt als Einflußseitenintensität angesetzt. Der Iterationsprozeß wurde solange wiederholt, bis die berechneten Intensitäten im gesamten Rechengebiet folgende Bedingung erfüllten:

$$
\left|I_{\text {neu }}-I_{\text {alt }}\right| \leq \epsilon \cdot \max \left\{\left|I_{\text {alt }}\right|\right\}
$$

( $I_{\text {alt }}$ : Intensität des Iterationsschritt $i-1, I_{\text {neu }}$ : Intensität des Iterationsschritt $i$ ). Die Konstante $\epsilon$ ist in den Rechnungen zu $\epsilon:=10^{-2}$ gesetzt worden. Für $\epsilon$-Werte von $10^{-3}$ und $10^{-4}$ zeigten sich im Vergleich zu $10^{-2}$ keine Unterschiede in den berechneten Intensitäten. Bei größeren $\epsilon-$ Werten $\left(\epsilon=10^{-1}\right)$ hingegen war die Anzahl der Iterationen pro Strahlungsrichtung größer als bei einem $\epsilon$-Wert von $10^{-2}$. 


\subsection{Numerische Resultate}

Die entwickelte ESC-Methode wurde anhand von 3 Testproblemen

- einem Problem mit einer vorgegebenen Lösung,

- dem 'Searchlight'-Problem,

- einer magnetischen Flußschicht in der solaren Schichtung,

auf Effizienz und Genauigkeit getestet. Die dazu benutzten numerischen Schemata zur Lösung der Gl. (4.15) entlang einer Charakteristik sind in Tab. 4.1 aufgelistet und kurz erläutert.

Tabelle 4.1: In der ESC-Methode eingesetzte numerische Schemata. ESC-Methoden erster Ordnung

ESC1(KA1)* linearer Ansatz + Kunasz-Auer-Löser mit linearer Approximation

ESC1(KA2)** linearer Ansatz + Kunasz-Auer-Löser mit quadratischer Approximation

ESC1(IRK) linearer Ansatz + impliziter 2-Punkt-Runge-Kutta-Löser

ESC1(DRK) linearer Ansatz + diagonal impliziter 2-Punkt-Runge-Kutta-Löser

ESC2(KA1) quadratischer Ansatz + Kunasz-Auer-Löser mit linearer Approximation

ESC2(KA2) quadratischer Ansatz + Kunasz-Auer-Löser mit quadratischer Approximation

ESC2(IRK) quadratischer Ansatz + impliziter 2-Punkt-Runge-Kutta-Löser

ESC2(DRK) quadratischer Ansatz + diagonal impliziter 2-Punkt-Runge-Kutta-Löser

ESC2-mod wie ESC2(IRK) allerdings mit der Oszillationskorrektur 4.2.3

$$
\text { Referenzmethoden ([52]) }
$$

DG0 Discontinuous-Galerkin-Methode mit konstanter Ansatzfunktion

DG1 Discontinuous-Galerkin-Methode mit linearer Ansatzfunktion

${ }^{*}$ ESC1(KA1) ist identisch mit der von Kunasz\& Auer ([47]) vorgeschlagenen Methode.

** ESC1(KA2) ist identisch mit der von Bruls et al. ([4]) vorgeschlagenen Methode. 


\subsubsection{Testproblem mit einer vorgegebenen exakten Lösung}

Analytische Funktionen der Intensität $I_{e x}(x, z)$ und $\chi(x, z):=\kappa(x, z) \rho(x, z)$ wurden für zwei Strahlungsrichtungen $\vec{\mu}$ ( $\alpha=50^{\circ}$ und $\alpha=200^{\circ}$ zur Horizontalen) vorgegeben (Gln. 4.17, Abb. 4.7). $S(x, z, \vec{\mu})$ und die Intensität am Einflußrand $g$ wurden so bestimmt, daß die Lösung der Strahlungstransportgleichung die vorgegebene Intensität $I_{e x}(x, z)$ zum Resultat hat. $\chi(x, z)$ wurde so gewählt (Abb. 4.7 rechts), daß es annährend die Bedingungen der späteren Anwendung (starken Variation von $\kappa \rho$ im Rechengebiet $10^{-3}-10^{4}$ ) wiederspiegelt

$$
\begin{aligned}
I_{e x}(x, z) & :=(\cos (2 \pi x)+2) \sin (\pi z)^{2}+1, \\
\chi(x, z) & :=(1000 \tanh (-5 z)+1000.001)(\sin (\pi x)+1.25) .
\end{aligned}
$$

Die Strahlungstransportgleichung Gl. (4.15) wurde mit der ESC-Methode und den
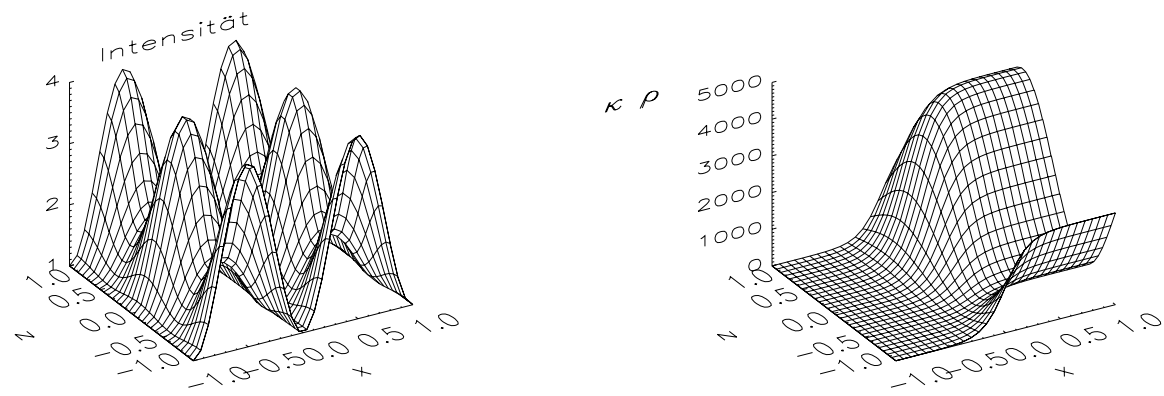

Abbildung 4.7: Analytische Funktionen für die Intensität I (links) und $\kappa \rho$ (rechts).

in Tabelle 4.1 aufgelisteten Schemata gelöst und die berechneten Resultate $I_{h}$ [Gl. (4.12)], welche Funktionen der Gitterweite $h$ des numerischen Gitters sind, mit der exakten Lösung $I_{e x}$ verglichen. Insofern $I_{e x}, S$ und $\kappa \rho$ ausreichend glatt, d.h. beliebig oft stetig differenzierbar im betrachteten Gebiet $\Omega$ sind, lassen sich Aufschlüsse über das Konvergenzverhalten der berechneten Lösung $I_{h}$ für $h \rightarrow 0$ und somit der Genauigkeit des Verfahrens auf einem vorgegeben Gitter gewinnen.

Im Allgemeinen kann dazu angenommen werden, daß der absolute Fehler einer numerischen Methode als asymptotische Reihe entwickelbar ist und folgende Form hat:

$$
e_{h}=c_{1} h^{p_{1}}+c_{2} h^{p_{2}}+c_{3} h^{p_{3}}+\ldots .+c_{n} h^{p_{n}}
$$

mit den reelen Exponenten $p_{i}$ für $i=1, . ., n$ und $p_{1}<p_{2}<p_{3}<\ldots<p_{n}$.

$p_{1}$ definiert dabei die Ordnung des numerischen Fehlers und somit die experimentell bestimmte Konvergenzordnung (EOC) der numerischen Methode für $h \rightarrow 0$. Zur experimentellen Bestimmung von $p_{1}$ benötigt man die ausreichend glatte, d.h. beliebig oft stetig differenzierbare exakte Lösung $I_{e x}$ eines Problems und die Fehler $e_{h}, e_{2 h}$ zwischen der exakten Lösung $I_{e x}$ und der numerischen $I_{h}$. Im aktuellen Fall 
wurde der $L^{2}-$ Fehler ([53]) verwendet:

$$
e_{h}:=\left(\int_{\Omega}\left|I(\vec{x})-I_{h}(\vec{x})\right|^{2} d \vec{x}\right)^{\frac{1}{2}}
$$

mit seiner diskreten Form:

$$
e_{h}:=A\left(\sum_{i=1, \cdots, n}\left|I^{(i)}(\vec{x})-I_{h}^{(i)}(\vec{x})\right|^{2}\right)^{\frac{1}{2}}
$$

$A$ ist hier der Flächeninhalt eines Dreieckselements. $n$ entspricht der Gesamtzahl von Punkten in einem Element, an denen der Fehler berechnet wird. Im Falle der ESC1-Methode sind dies die Dreiecksknoten, an denen auch die Koeffizienten $I_{j}$ [Gl. (4.13)] berechnet wurden (Abb. 4.5 links), und es gilt $n=3$. Bei der ESC2Methode sind dies die Knoten und die Kantenmittelpunkte und es ist $n=6$ (Abb. 4.5 Mitte und rechts). Unter den Annahmen, daß $c_{i}=$ const. ist und für die Fehlernormen $\left\|e_{h}\right\|=c h^{p_{1}}$ und $\left\|e_{2 h}\right\|=c(2 h)^{p_{1}}$ gilt, folgt für das Verhältnis der Fehler:

$$
\frac{\left\|e_{2 h}\right\|}{\left\|e_{h}\right\|}=\frac{c(2 h)^{p_{1}}}{c h^{p_{1}}}=2^{p_{1}}
$$

woraus sich die Konvergenzordnung ergibt:

$$
p_{1}=\log _{2} \frac{\left\|e_{2 h}\right\|}{\left\|e_{h}\right\|}
$$

Für den $E O C$ gilt damit:

$$
E O C=\lim _{h \rightarrow 0} p_{1}
$$

Die winkelgemittelte Intensität (2.44), welche für den Strahlungsquellterm (2.42) benötigt wird, erfordert die Berechnung der Intensität für verschiedene Winkelrichtungen. Um eine mögliche Abhängigkeit des $E O C$ von der Strahlungsrichtung erkennen zu können, sind alle Konvergenzuntersuchungen für zwei unterschiedliche Strahlungsrichtungen durchgeführt worden. Eine aufwärtsgerichtete $\left(\mu_{z}>0\right.$, $\alpha=50^{\circ}$ zur Horizontalen) und eine abwärtsgerichtete $\left(\mu_{z}<0, \alpha=200^{\circ}\right.$ zur Horizontalen). Eine Untersuchung bezüglich anderer Winkelrichtungen wurde durchgeführt und bekräftigte die für $\alpha=50^{\circ}$ und $\alpha=200^{\circ}$ erzielten Resultate, weshalb im weiteren nur die Resultate für diese beiden Richtungen diskutiert werden.

In Abb. 4.8 ist der EOC der ESC1-Methode für die beiden Winkelrichtungen dargestellt. Während die ESC1(KA1)- und ESC1(KA2)-Löser einen geringfügig grösseren $E O C$ bei $\alpha=50^{\circ}$ haben als die Runge-Kutta-Löser, lässt sich dagegen ein deutlich konvergenteres Verhalten bei den ESC1(IRK)- und ESC1(DRK)Lösern erkennen. Im Falle von $\alpha=200^{\circ}$ ist der Unterschied zwischen den vier Lösertypen nur noch marginal. Auch bei den ESC1(KA1)- und ESC1(KA2)-Lösern ist eine Konvergenz deutlicher zu erkennen. 
In Abb. 4.9 ist der EOC der ESC2-Methode für die beiden Winkelrichtungen dargestellt. Im Fall des $\alpha=50^{\circ}$ Winkels offenbarte der ESC2(IRK)-Löser die am stärksten ausgeprägte Konvergenz. Der $E O C$ startet bei einem Wert größer als 2.5 erzielt mit 16384 Gitterzellen und nähert sich für $2 \cdot 10^{6}$ Gitterlementen dem Wert 2.0, während sich die anderen Löser nicht sehr stark voneinander unterscheiden. Bei der Starttriangulierung mit 16384 Gitterzellen starten sie zwischen 2.0 und 2.2 und liegen zwischen 2.0 und 2.2 immer noch bei $2 \cdot 10^{6}$ Gitterzellen. Beim $\alpha=200^{\circ}$ Winkel hingegen sind sich die Verhalten des ESC2(IRK)- und des ESC2(DRK)Lösers qualitativ sehr ähnlich. Der $E O C$ startet bei 2.3 und liegt bei $2 \cdot 10^{6}$ Gitterzellen zwischen 2.0 und 2.1, während der $E O C$ der ESC2(KA1)- und ESC2(KA2)-Löser unter 2.0 liegt und noch nicht ersichtlich ist, zu welchem Wert er konvergieren wird.

Die EOC der Referenzverfahren DG0 und DG1 haben sowohl bei der Starttriangulierung mit 16384 Gitterzellen, als auch bei $2 \cdot 10^{6}$ Gitterlementen einen Wert der beim DG0-Verfahren bei 1.0 liegt und beim DG1-Verfahren bei 2.0, unabhängig von der Winkelrichtung.

Abbildung 4.10 zeigt die wesentlichen Unterschiede zwischen den einzelnen Resultaten. Die Laufzeitunterschiede zwischen den ESC2-Lösern sind sehr groß. Die ESC2(KA1)- und ESC2(KA2)-Löser benötigen etwa $10^{2}$ mal länger, um einen gegebenen Fehler zu unterschreiten, da sie dazu eine wesentlich kleinere Gitterweite benötigen, als die verwendeten Runge-Kutta-Löser, welche einen vorgeschriebenen Fehler auf einem deutlich gröberen Gitter erreichen. Selbst die DiscontiuousGalerkin-Referenzverfahren schneiden deutlich schlechter ab als die Runge-KuttaLöser. Die von uns verwendete Oszillationskorrektur scheint auf die Güte der Resultate keinen entscheidenden Einfluß zu haben. Der Vergleich des oszillatioskorrigierten impliziten Runge-Kutta-Löser (ESC2-mod) mit dem nicht korrigierten [ESC2(IRK)] macht deutlich, daß die angewandte Oszillationskorrektur bei gleichbleibenden Fehler die Berechnungszeit unwesentlich beeinflußt, während es unphysikalische Oszillationen beseitigt. Die Tabellen 4.2 und 4.3 zeigen die Berechnungszeiten, welche die einzelnen Löser benötigen, um einen vorgeschriebenen Fehler zu unterschreiten. Zusammenfassend kann gesagt werden, daß die ESC2-Methode mit dem oszillationskorrigierten impliziten Runge-Kutta-Löser (ESC2-mod), die beste der untersuchten Methoden ist. Sie ist von 2. Ordnung genau, erreicht eine geforderte Genauigkeit schon mit bedeutend größerer Gitterweite $h$, ist somit sehr viel schneller und verursacht keine Oszillationen. 

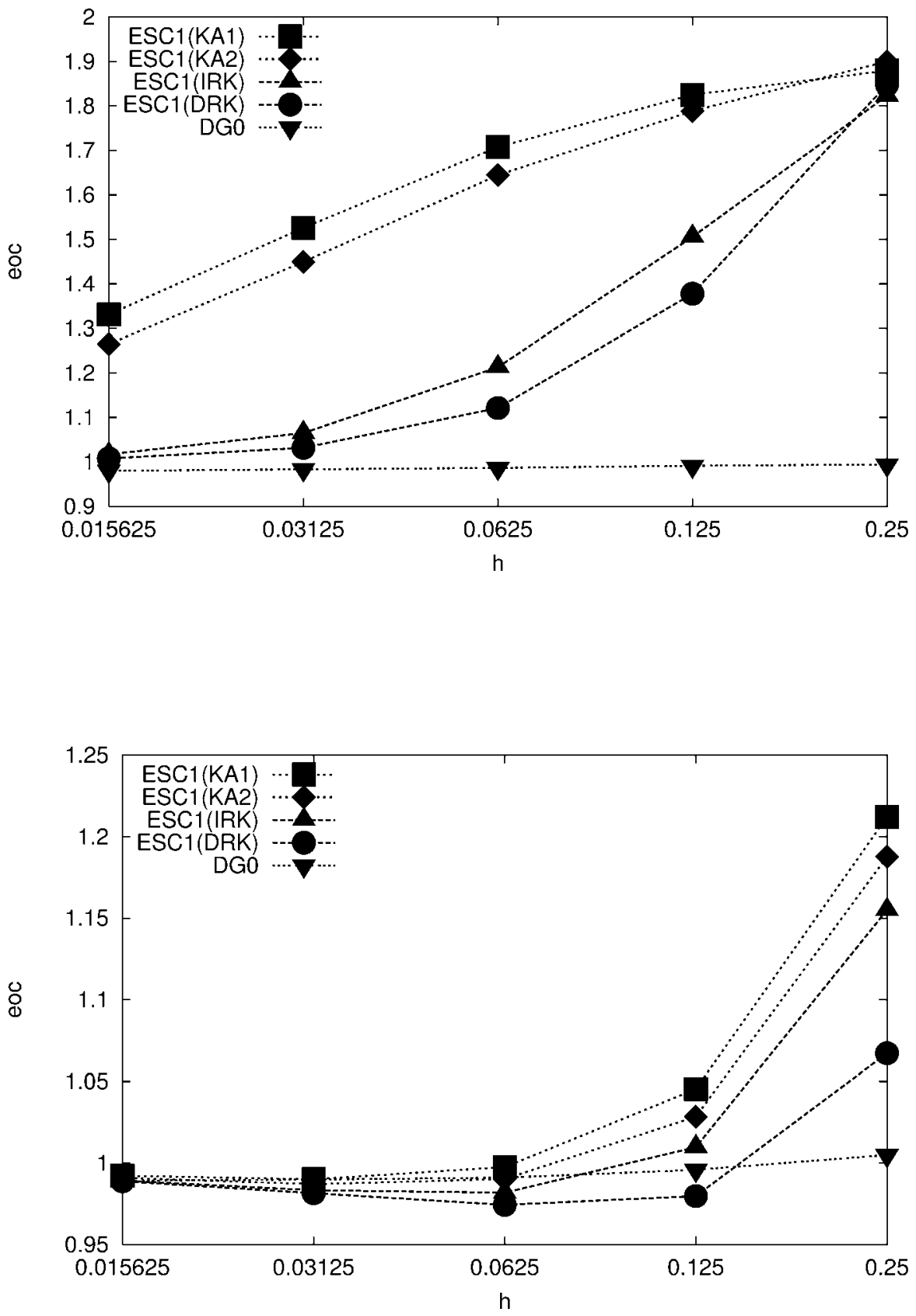

Abbildung 4.8: $E O C$ als Funktion der Gitterweite $h$ (willkürliche Einheiten) für die ESC1-Methode, welcher unter Benutzung der in Tabelle 4.1 aufgelisteten Verfahren ermittelt worden ist. Links: Winkel von $\alpha=50^{\circ}$. Rechts: Winkel von $\alpha=200^{\circ}$. 

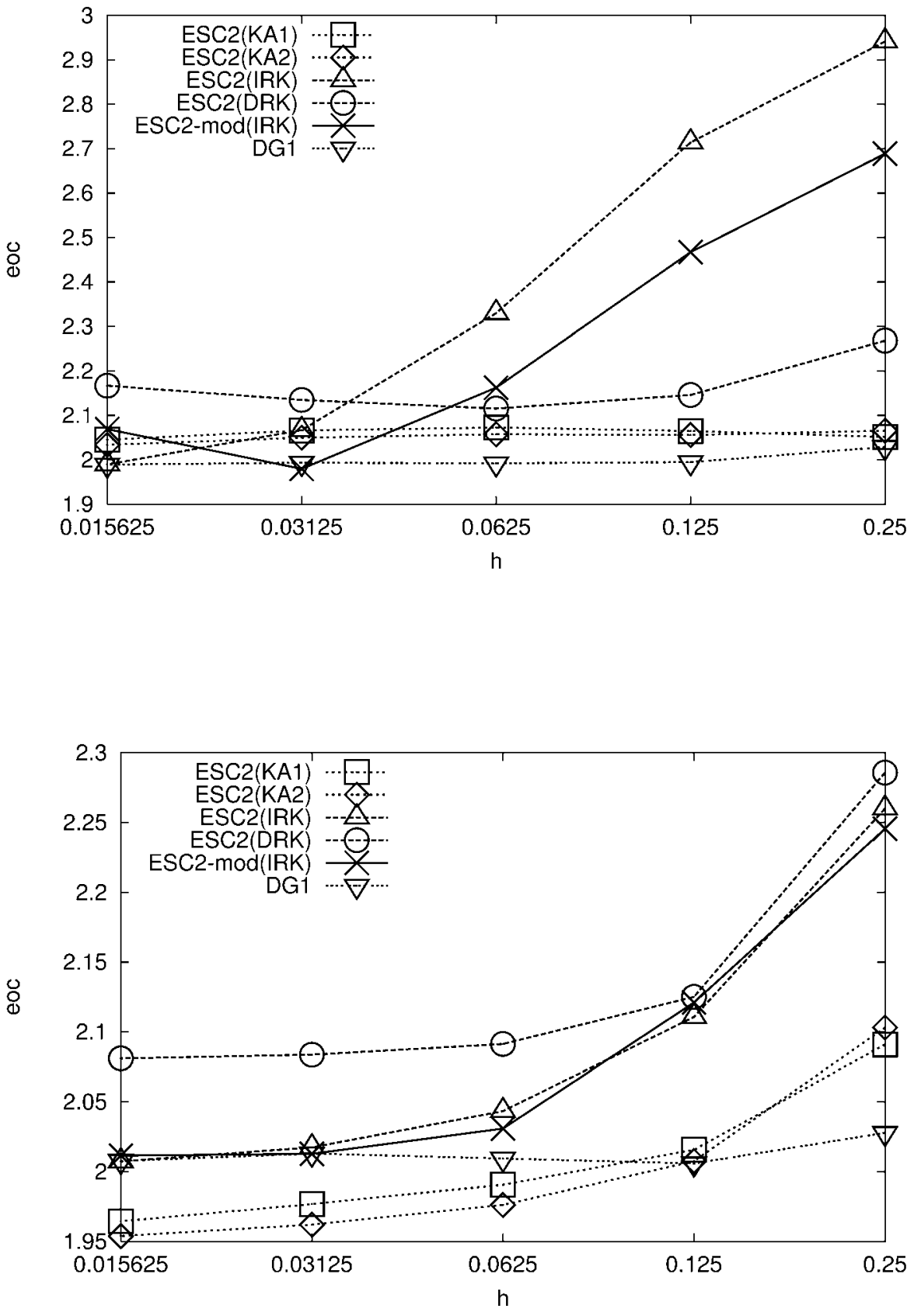

Abbildung 4.9: $E O C$ als Funktion der Gitterweite $h$ (willkürliche Einheiten) für die ESC2-Methode, welcher unter Benutzung der in Tabelle 4.1 aufgelisteten Verfahren ermittelt worden ist. Links: Winkel von $\alpha=50^{\circ}$. Rechts: Winkel von $\alpha=200^{\circ}$. 

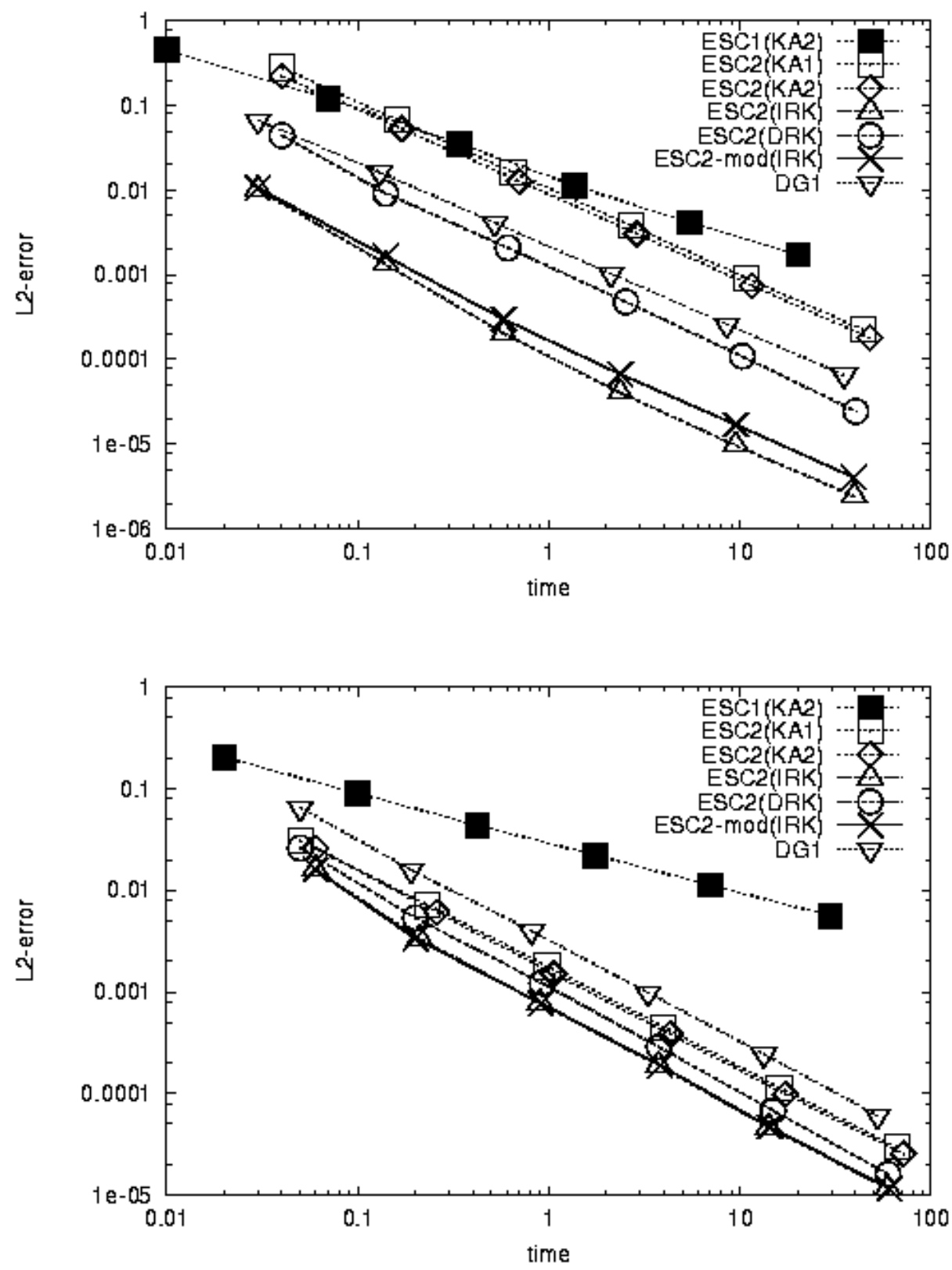

Abbildung 4.10: L2-Fehler als Funktion der Berechnungszeit $t$ (willkürliche Einheiten). Die Berechnungszeit benötigen die in Tabelle 4.1 aufgelisteten Verfahren, um einen vorgeschriebenen $L 2-F e h l e r$ zu unterschreiten. Links: Winkel von $\alpha=50^{\circ}$. Rechts: Winkel von $\alpha=200^{\circ}$. 


\begin{tabular}{rrrrrr}
\hline$e_{h}$ & ESC1(KA1) & $\begin{array}{r}\text { ESC1(KA2) } \\
\text { Winkel } \alpha=50^{\circ}\end{array}$ & $\begin{array}{c}\text { ESC1(IRK) } \\
\text { ESC1(DRK) }\end{array}$ & DG0 \\
\hline $1.00 \mathrm{e}-02$ & $8.93 \mathrm{e}-01$ & $3.05 \mathrm{e}-02$ & $1.31 \mathrm{e}-01$ & $3.17 \mathrm{e}-02$ & $2.14 \mathrm{e}-01$ \\
$1.00 \mathrm{e}-03$ & $8.63 \mathrm{e}+00$ & $1.74 \mathrm{e}-01$ & $1.24 \mathrm{e}+00$ & $2.13 \mathrm{e}-01$ & $2.19 \mathrm{e}+00$ \\
$1.00 \mathrm{e}-04$ & $8.66 \mathrm{e}+01$ & $1.07 \mathrm{e}+00$ & $1.11 \mathrm{e}+01$ & $1.62 \mathrm{e}+00$ & $2.27 \mathrm{e}+01$ \\
$1.00 \mathrm{e}-05$ & $8.75 \mathrm{e}+02$ & $9.14 \mathrm{e}+00$ & $9.06 \mathrm{e}+01$ & $1.61 \mathrm{e}+01$ & $2.41 \mathrm{e}+02$ \\
\hline \multicolumn{7}{c}{ Winkel $\alpha=200^{\circ}$} \\
\hline $1.00 \mathrm{e}-02$ & $1.57 \mathrm{e}-01$ & $8.75 \mathrm{e}-02$ & $1.16 \mathrm{e}-01$ & $8.60 \mathrm{e}-02$ & $3.09 \mathrm{e}-01$ \\
$1.00 \mathrm{e}-03$ & $1.62 \mathrm{e}+00$ & $6.98 \mathrm{e}-01$ & $1.12 \mathrm{e}+00$ & $6.97 \mathrm{e}-01$ & $3.26 \mathrm{e}+00$ \\
$1.00 \mathrm{e}-04$ & $1.70 \mathrm{e}+01$ & $6.83 \mathrm{e}+00$ & $1.03 \mathrm{e}+01$ & $6.98 \mathrm{e}+00$ & $3.22 \mathrm{e}+01$ \\
$1.00 \mathrm{e}-05$ & $1.91 \mathrm{e}+02$ & $6.86 \mathrm{e}+01$ & $9.37 \mathrm{e}+01$ & $7.05 \mathrm{e}+01$ & $3.10 \mathrm{e}+02$ \\
\hline
\end{tabular}

Tabelle 4.2: Laufzeiten (willkürliche Einheiten) der Verfahren erster Ordnung, nach denen ein gegebener L2-Fehler unterschritten wird.

\begin{tabular}{rrrrrr}
\hline & & & \\
$e_{h}$ & ESC2(KA2) & ESC2(IRK) & ESC2(DRK) & ESC2-mod(IRK) & DG1 \\
\hline \multicolumn{7}{c}{ Winkel $\alpha=50^{\circ}$} \\
\hline $1.00 \mathrm{e}-02$ & $8.93 \mathrm{e}-01$ & $3.05 \mathrm{e}-02$ & $1.31 \mathrm{e}-01$ & $3.17 \mathrm{e}-02$ & $2.14 \mathrm{e}-01$ \\
$1.00 \mathrm{e}-03$ & $8.63 \mathrm{e}+00$ & $1.74 \mathrm{e}-01$ & $1.24 \mathrm{e}+00$ & $2.13 \mathrm{e}-01$ & $2.19 \mathrm{e}+00$ \\
$1.00 \mathrm{e}-04$ & $8.66 \mathrm{e}+01$ & $1.07 \mathrm{e}+00$ & $1.11 \mathrm{e}+01$ & $1.62 \mathrm{e}+00$ & $2.27 \mathrm{e}+01$ \\
$1.00 \mathrm{e}-05$ & $8.75 \mathrm{e}+02$ & $9.14 \mathrm{e}+00$ & $9.06 \mathrm{e}+01$ & $1.61 \mathrm{e}+01$ & $2.41 \mathrm{e}+02$ \\
\hline \multicolumn{7}{c}{ Winkel $\alpha=200^{\circ}$} \\
\hline $1.00 \mathrm{e}-02$ & $1.57 \mathrm{e}-01$ & $8.75 \mathrm{e}-02$ & $1.16 \mathrm{e}-01$ & $8.60 \mathrm{e}-02$ & $3.09 \mathrm{e}-01$ \\
$1.00 \mathrm{e}-03$ & $1.62 \mathrm{e}+00$ & $6.98 \mathrm{e}-01$ & $1.12 \mathrm{e}+00$ & $6.97 \mathrm{e}-01$ & $3.26 \mathrm{e}+00$ \\
$1.00 \mathrm{e}-04$ & $1.70 \mathrm{e}+01$ & $6.83 \mathrm{e}+00$ & $1.03 \mathrm{e}+01$ & $6.98 \mathrm{e}+00$ & $3.22 \mathrm{e}+01$ \\
$1.00 \mathrm{e}-05$ & $1.91 \mathrm{e}+02$ & $6.86 \mathrm{e}+01$ & $9.37 \mathrm{e}+01$ & $7.05 \mathrm{e}+01$ & $3.10 \mathrm{e}+02$ \\
\hline
\end{tabular}

Tabelle 4.3: Laufzeiten (willkürliche Einheiten) der Verfahren zweiter Ordnung, nach denen ein gegebener L2-Fehler unterschritten wird. 


\subsubsection{Searchlight-Problem}

Die Annahme von Polynomen erster oder zweiter Ordnung für die Basisfunktionen auf jedem Element liefert die Startintensitäten für jede short-characteristic. Dies entspricht, wie in Kap. 4.2.3 bereits erläutert wurde, einer linearen bzw. quadratischen Interpolation der Koeffizienten (Gl. (4.12)) an den Startpunkten der Charakteristiken. Diese Interpolation, die auf jeder Einflußseite eines Gitterelements durchgeführt wird, bewirkt eine in Strahlrichtung zunehmende Verschmierung der Intensitäten senkrecht zur Strahlrichtung. Im Falle eines scharf begrenzten Strahls (Searchlight), führt dieser Effekt, auch im Falle verschwindender Absorption zu einer Intensitätsdiffusion aus dem Strahl in dessen Umgebung, was eine Intensitätsabnahme im Strahl selber bewirkt. Die Abbildung 4.11 zeigt solche Strahlenbündel, für (von links nach rechts) die exakte Lösung, die ESC1(KA2)-, die ESC2(IRK)und die DG1-Methode. Die Intensitätsdiffusion für die ESC1-Methode ist am deutlichsten, während die ESC2(IRK)- und die DG1-Methode kaum Diffusion zeigen. Dafür neigen sie zur Oszillationsbildung. In Abbildung 4.12 ist ein horizontaler Schnitt am oberen Rechengebietsrand durch die Strahlenbündel, welche mit den in Tab. 4.1 aufgeführten Methoden berechnet wurden, im Vergleich zur exakten Lösung dargestellt. Deutlich zu erkennen sind die stark diffusiven Eigenschaften der ESC1(KA2)-Methode und das oszillatorische Verhalten der ESC2(IRK)- und der DG1-Methode. Wie bereits erwähnt lassen sich diese Oszillationen im Falle der ESC(IRK)-Methode leicht eliminieren, was zur ESC-mod-Methode führt, ohne daß an der Strahlstruktur und dem scharfen Strahlrand etwas verändert wird.
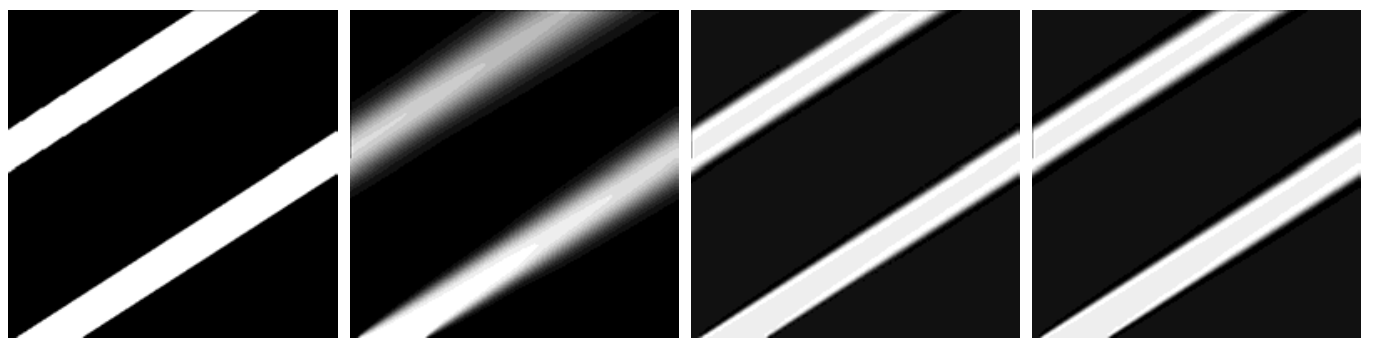

Abbildung 4.11: Graustufen Darstellungen der Intensität eines sogenannten SearchlightStrahls, der sich in Vakuum $(\kappa=0)$ ausbreitet. Das numerische Gitter besteht aus 42478 Gitterzellen. Von links nach rechts: Exakte Lösung und Resultate, die mit der ESC1(KA2), der ESC2(IRK)- und der DG1-Methode ermittelt wurden. Der Strahl gelangt durch den unteren Rand in das Rechengebiet, breitet sich unter einem Winkel von $\alpha=33^{\circ}$ zur Horizontalen aus und verläßt das Gebiet in der Mitte des oberen Randes. Der Strahl durchläuft das Gebiet zweimal, was aus der in 4.2.4 beschriebenen periodischen seitlichen Randbedingungen resultiert. 


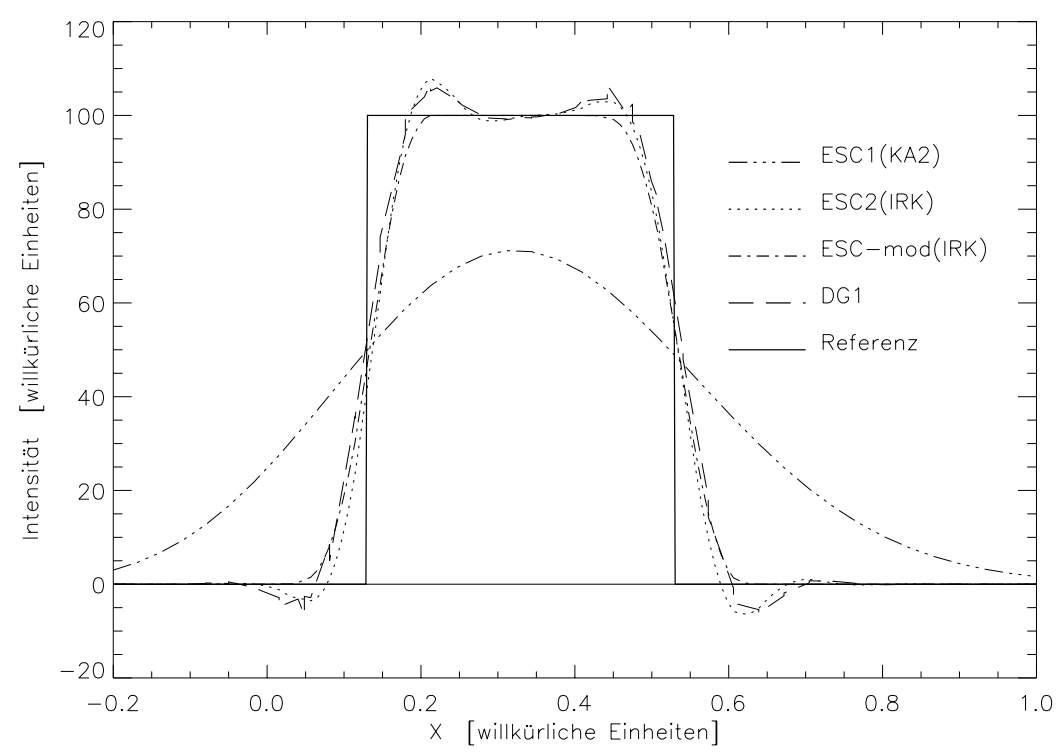

Abbildung 4.12: Die quadratische Interpolation kann neue Extrema erzeugen und zu unphysikalischen negativen Intensitäten führen. Dies wird durch einen direkten Vergleich der mit den nachfolgend aufgelisteten Methoden berechneten Intensitäten am oberen Gebietsrand deutlich: ESC2(IRK), ESC1(KA2), DG1, ESC2-mod. Das mit der ESC1(KA2)Methode erzielte Resultat zeigt die stärkste Diffusion, wohingegen die Methoden höherer Ordnung zur Oszillationsbildung neigen (ESC2(IRK), DG1), die im Fall der ESC2(IRK)Methode leicht eliminierbar sind und zur ESC2-mod-Methode führt.

\subsubsection{Solare magnetische Flußschicht: Ein realitätsnaher Test- fall}

An der Sonnenoberfläche beobachtbare magnetische Flußkonzentrationen bestehen aus mehr oder weniger stark ausgedehnten Ansammlungen vertikalen magnetischen Feldes. Der Großteil des magnetischen Flusses und beinahe die gesamte magnetische Energie ist in sogenannten Flußröhren gebündelt. Die bislang beobachtbaren räumlichen Ausdehnungen dieser Flußröhren reicht von mehreren zehntausend Kilometern Durchmesser, bei Sonnenflecken, bis hinunter zu Flußkonzentrationen mit einem Durchmesser von einigen 100 Kilometern wie von Solanki (1992) und Stolpe\&Kneer (1997, 2000) beschrieben ([80], [93], [94]). Im aktuellen Fall liegt das Hauptaugenmerk auf der dynamischen Wechselwirkung von Magnetfeld und solarer Konvektion, die von Steiner et al. (1988) ([90]) mittels MHD-Simulationen untersucht wurde. Da solaren Bereichen um $\tau_{5000}=1$ der Strahlungstransport die Konvektion als dominierenden Energietransportmechanismus ablöst, wird die Dynamik der Magnetfeldkonzentrationen in diesen Regionen auch durch den Strahlungstransport maßgeblich beeinflußt. Knölker et al. (1988) ([45]) leiteten anhand von 2D-MHD-Simulationen typische Längen- und Zeitskalen, auf denen die energetische Interaktion von kleinskaligen magnetischen Flußkonzentrationen und dem Stahlungsfeld der sie umgebenden Gebieten abläuft, her. Um nun die Interaktion von solchen kleinräumigen Magnetfeldkonzentrationen und dem radiativen Strahlungsfeld verstehen zu können, wurde als ein erster Schritt das Modell einer ma- 
gnetischen Flußschicht in das Modell der ruhigen Sonnenphotosphäre, in nachfolgend erläuterter Weise, eingebettet ([43], [44], [2], [4]). Um die betrachteten Löser in einer realitätsnahen Anwendung zu untersuchen, wurde die Strahlungstransportgleichung anschließend für einen Winkel $\alpha=80^{\circ}$ zur Horizontalen für dieses Flußschichtmodell gelöst.

- Als Modell für den Bereich außerhalb der Flußschicht wurde das in Kap. 2.9 .5 bereits erläuterte eindimensionale Modell der ruhigen Photosphäre und Konvektionszone von Spruit ([83]) verwendet.

- Innerhalb der Flußschicht wurde das Modell des äußeren Bereichs um eine "Wilson-depression" von $\Delta z=200 \mathrm{~km}$ nach unten verschoben.

- Unter der Annahme, daß $\frac{d}{H} \ll 1$ ist kann die Näherung dünner Flußröhren ([68]) angewendet werden :

$$
p_{e}(z)=p_{i}(z)+\frac{B^{2}(z)}{8 \pi}
$$

( $e \equiv$ extern, $i \equiv$ intern, $H_{p}$ : Druckskalenhöhe, $d$ : Dicke der Flußschicht, $p_{e}$ : Gasdruck im äußeren Gebiet, $p_{i}$ : Gasdruck in der Flußschicht, $B$ : vertikale Magnetfeldkomponente).

- Magnetische Flußerhaltung fordert:

$$
\hat{\Phi}_{\text {mag }}=\int_{0}^{d} B \mathrm{~d} x=B(z) d(z)=\text { const } .
$$

- damit folgt die Geometrie der Flußschicht :

$$
\begin{gathered}
\longrightarrow B_{0} d_{0}=B(z) d(z) \\
\left(d_{0}=d_{z_{w i l s}}=200 \mathrm{~km}, \quad B_{0}=\sqrt{8 \pi\left(p_{e}\left(z_{w i l s}\right)-p_{i}\left(z_{w i l s}\right)\right)}\right), \\
\Longrightarrow d(z)=\left(8 \pi\left(p_{e}(z)-p_{i}(z)\right)^{-1 / 2} B_{0} d_{0}\right.
\end{gathered}
$$

$\left(\frac{B^{2}}{8 \pi}\right.$ : magnetischer Druck, $z_{\text {wils }}$ : ist die geometrische Höhe des $\tau_{5000}=1$ Niveaus innerhalb der Flußschicht).

Dabei ist die Quellfunktion $S$ die frequenzintegrierte Planck-Funktion Gl. 4.6 und die Absorption wird durch den Rosselandschen Absorptionkoeffizienten Gl. (4.4) beschrieben. Um über das Verhalten der Löser bei starken Gradienten in den hydrodynamischen Variablen, wie sie auch bei Stoßfronten auftauchen können, einen Aufschluß zu erhalten, wird ein sehr scharfer Übergang an den Rändern der Flußschicht angenommen (Abb. 4.13). Das qualitative Resultat ist in Abb. 4.14 dargestellt. Am oberen Gebietsrand, der ca. $500 \mathrm{~km}$ über $\tau_{5000}=1$ liegt, sind zwei 

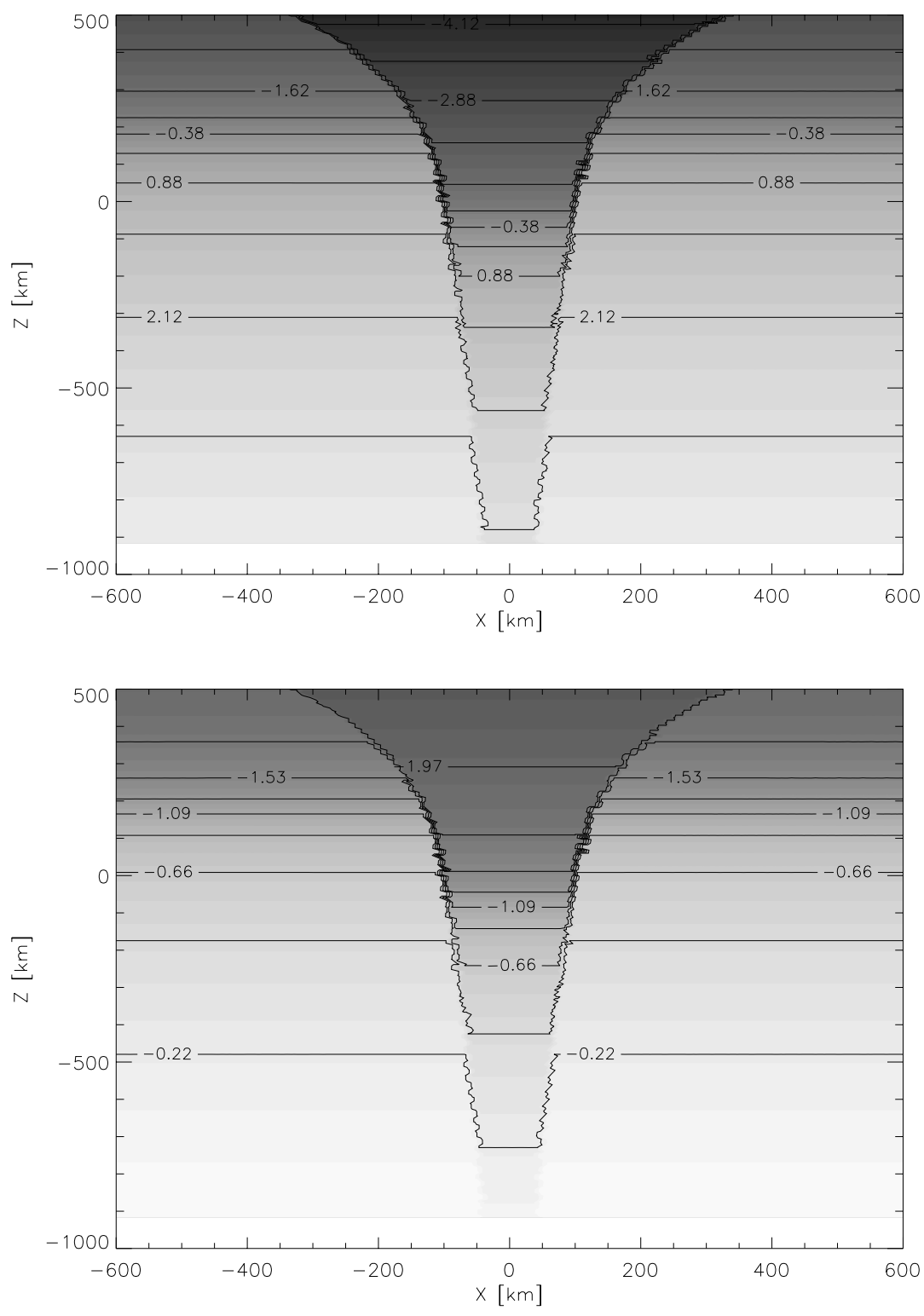

Abbildung 4.13: Oben: Kombination einer Graustufen- und einer Isoliniendarstellung von $\log _{10}(\kappa \rho)$. Unten: Kombination einer Graustufen- und einer Isoliniendarstellung von $\log _{10} S$. Beide Größen nehmen mit zunehmender geometrischer Tiefe zu und zeigen einen scharfen Übergang an den Flußschichträndern.

Intensitätsmaxima erkennbar. Ihre Existenz läßt sich dadurch verstehen, daß die Flußschichtatmosphäre auf gleicher geometrischer Höhe kühler als die externe Atmosphäre ist und eine geringere Dichte hat. Daraus folgt, daß in der Höhe $z$ in der $\tau_{e, 5000}=1$ ist, $\tau_{i, 5000} \ll 1$ und $\kappa_{e} \rho_{e} \gg \kappa_{i} \rho_{i}$ sind. Die mittlere freie Weglänge der Photonen außerhalb der Flußschicht $l_{e}=\left(\kappa_{e} \rho_{e}\right)^{-1}$ ist in einer bestimmten geometrischen Höhe $z$ somit viel geringer als innerhalb. Da generell in Regionen mit $\tau_{5000} \ll$ 1 keine erwähnenswerte Absorption mehr stattfindet, ist die formale Lösung Gl. (4.16) der Gl. (4.14) auf den ersten Teil, der den Transport beschreibt, reduziert. Somit finden Photonen auf ihrer Wanderung von tieferen Schichten mit Überqueren 
des Flußschichtrandes plötzlich ein Gebiet mit sehr geringer Absorption vor und können beinahe ungestört bis zum oberen Rand weiterwandern. Abb. 4.15 zeigt

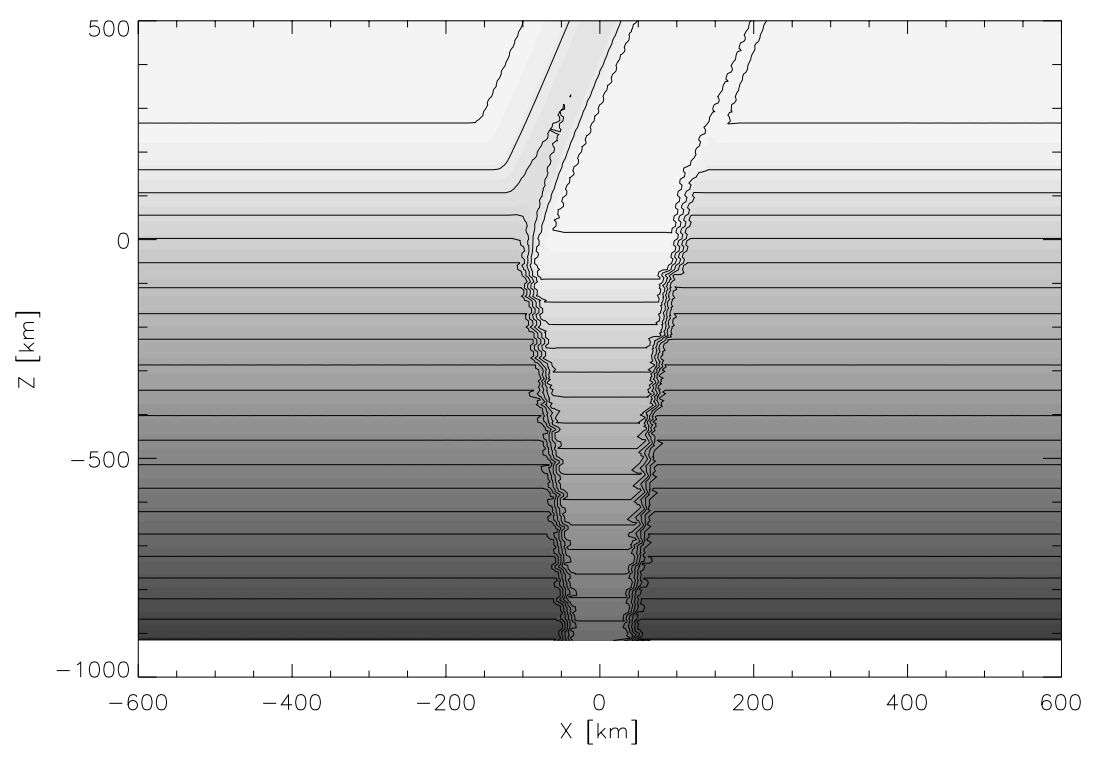

\begin{abstract}
Abbildung 4.14: Die Spezifische Intensität für einen Winkel von $80^{\circ}$ zur Horizontalen, im Falle der magnetischen Flußschicht, als Kombination einer Isolinien- und einer Graustufendarstellung. Sie wurde mit der ESC2-mod-Methode und 135644 Gitterzellen berechnet. Die Intensität nimmt mit zunehmender geometrischer Tiefe $z$ zu. Am oberen Gebietsrand (ca. $500 \mathrm{~km}$ über $\tau_{5000}=1$ ) sind zwei Intensitätsmaxima erkennbar (Fig. 4.15), die ihren Ursprung in Schichten um $\tau_{5000}=1$ haben.
\end{abstract}

das Konvergenzverhalten der berechneten Intensitäten am oberen Rechengebietsrand gegen eine Referenzlösung (berechnet mit der ESC2-mod-Methode und $10^{6}$ Gitterzellen) als Funktion der Gitterzellenanzahl. Die mit der ESC2-mod-Methode und der DG1-Methode berechneten Resultate zeigen schon mit 33770 Gitterzellen keine signifikanten Abweichungen zur Referenzlösung. Die ESC1(KA2)- und die ESC2(KA2)-Methode sind entweder sehr diffusiv oder produzieren Oszillationen, die aus dem short-characteristics-Löser herrühren und nicht durch die Intensitätsinterpolation bedingt sind. Die quantitative Beschreibung des Konvergenzverhaltens ist in Abb. 4.16 dargestellt. Sie zeigt das Verhältnis der Intensitätsmaxima am oberen Rand, welche mit dem Modell der ungestörten Photosphäre und dem die Magnetfeldkonzentration enthaltenden berechnet wurden. 

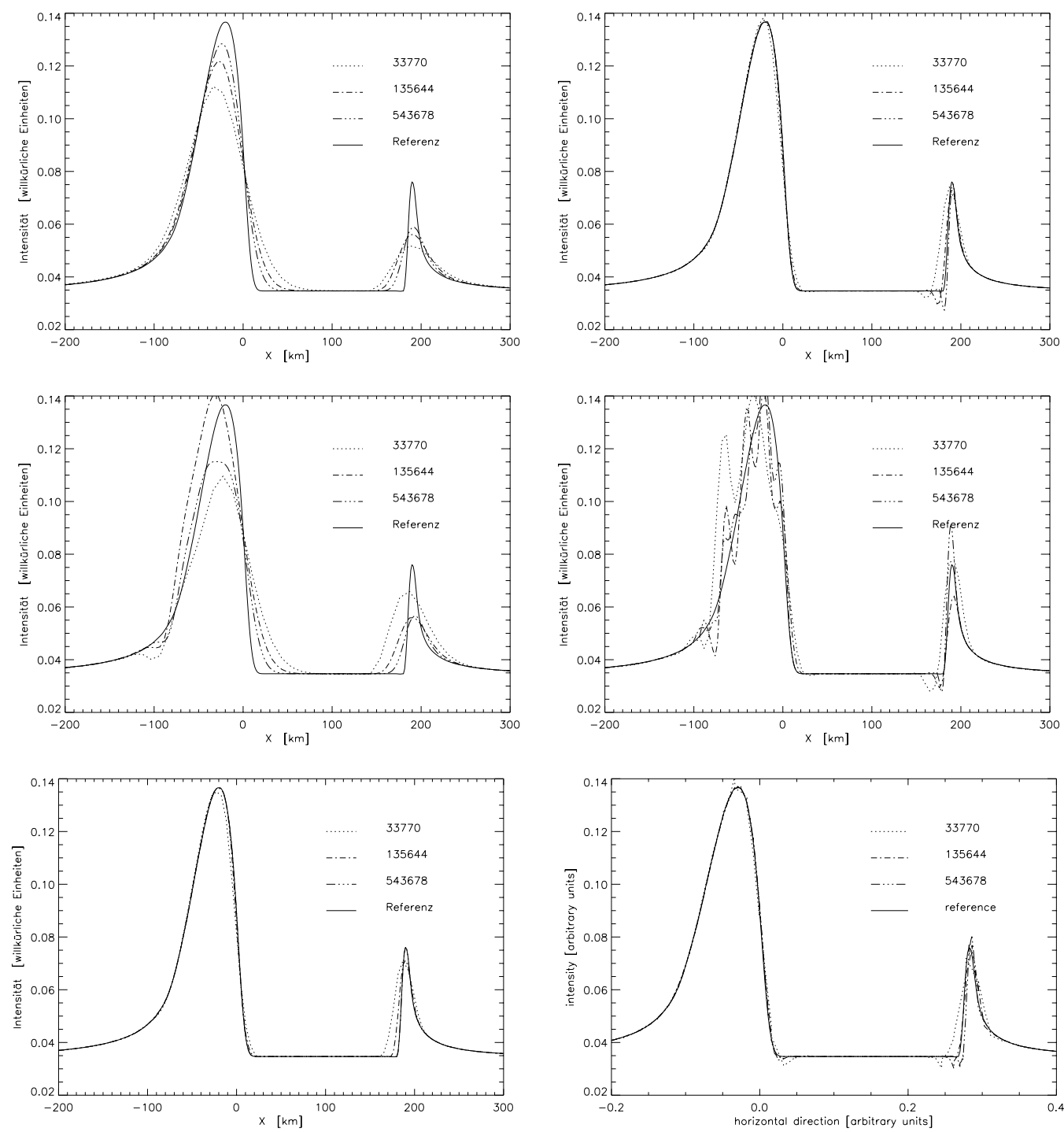

Abbildung 4.15: Vergleich berechneter Intensitäten am oberen Rand des Rechengebiets. Oben links und oben rechts: ESC1(IRK), ESC2(IRK). Mitte links und Mitte rechts: ESC1(KA2), ESC2(KA2). Unten links und unten rechts: ESC2-mod, DG1. Die Resultate wurden mit verschiedenen Gitterzellenanzahlen berechnet (33770, 135644, 543678). Die Referenzlösung wurde mit der ESC2-mod-Methode und $10^{6}$ Gitterzellen berechnet.

Zusammenfassend kann gesagt werden, daß die Anwendungen den ESC2-modLöser hervorheben. Dieser Löser wird in den zeitabhängigen Simulationsrechnungen Anwendung finden, da er das beste Fehler zu Laufzeitverhältnis und die geringste Intensitätsdiffusion hat und auch in dem Anwendungsfall der magnetischen Flußschicht die besten Resultate lieferte. 

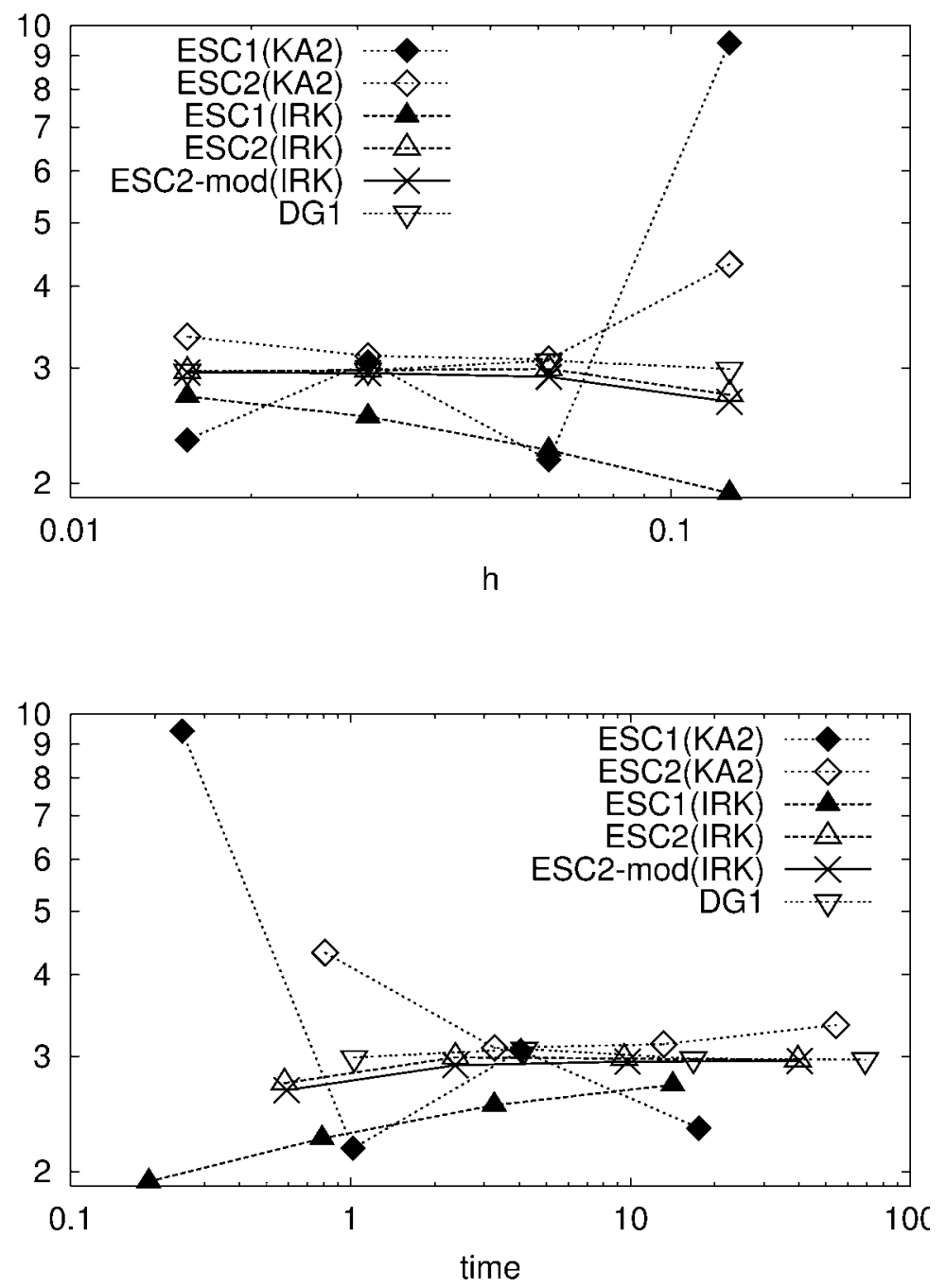

Abbildung 4.16: Oben: Das Verhältnis der Intensitätsmaxima am oberen Rand, welche mit dem Modell der ungestörten Photosphäre $\left(I_{e x}\right)$ und die Magnetfeldkonzentration enthaltenden $\left(I_{f l}\right)$ berechnet wurden, als Funktion der Gitterweite. Die DG1-, ESC2-mod-und die ESC2(IRK)-Methode zeigen vergleichbare Resultate und konvergieren für $h \rightarrow 0$ offensichtlich zum selben Wert. Die ESC1(KA2)-Methode and ESC2(KA2)-Methode zeigen ein sehr sprunghaftes Verhalten und bei $2 \cdot 10^{\varsigma}$ Elementen scheint noch nicht klar zu sein, $o b$ sie überhaupt konvergieren und wenn ob der Grenzwert mit dem Grenzwert der ersten drei Methoden übereinstimmt. Unten: $I_{f l} / I_{e x}$ als Funktion der Berechnungszeit. 


\subsection{Numerische Bestimmung des Strahlungsquellterms}

Der Energietransport durch Strahlung findet mittels des Quellterms (Kap. 2.7) in der Erhaltungsgleichung der Gesamtenergiedichte (Kap. 2.26) Eingang in das System der MHD-Gleichungen (Kap. 2.8). Es gibt zwei äquivalente Formulierungen von $Q_{\mathrm{R}}([4])$, die sich in der frequenzabhängigen Form folgendermaßen darstellen:

$$
\begin{aligned}
Q_{\mathrm{R}}(\vec{r}) & =4 \pi \rho \int_{\nu} \kappa_{\nu}\left(J_{\nu}-S_{\nu}\right) d \nu, \\
Q_{\mathrm{R}}(\vec{r}) & =\int_{\nu} \nabla \cdot \vec{F}_{\nu}^{r a d} d \nu .
\end{aligned}
$$

Beide Formulierungen verlangen die Bestimmung der spezifischen Intensitität $I_{\nu}(\vec{r}, \vec{\mu})$ durch Lösung der Strahlungstransportgleichung Gl. (4.1) für mehrere Raumrichtungen $\vec{\mu}$ und anschließend die Berechnung eines Raumwinkelintegrals. Dazu muß der Raumwinkelbereich durch eine Anzahl von diskreten Winkelrichtungen $\vec{\mu}$, über die dann integriert wird, diskretisiert werden. Ein Schema zur Ermittelung verschiedenster Raumwinkelquadraturen wurde von Carlson (1963) ([5]) hergeleitet. Im folgenden wird die aus 12 Winkelrichtungen bestehende $A 4$-Quadratur eingesetzt. Im Falle einer zeitabhängigen Simulationsrechnung ist der Einsatz einer Raumwinkelquadratur mit mehr als 12 Winkelrichtungen viel zu rechenzeitintensiv. Schon beim Einsatz der A4-Quadratur liegt die Berechnungszeit des Strahlungquellterms im Mittel eine Größenordnungen über der des MHD-Lösers. Im Falle von zweidimensionalen Simulationsrechnungen mit statischen Rechengittern und einer Gitteraufösung von $10 \mathrm{~km}$, was bei Rechengebietsgrößen von $12000 \mathrm{~km} \times 1500 \mathrm{~km}$ $1.8 \cdot 10^{5}$ Gitterzellen entspricht, erscheint dies gerade noch vertretbar. Im Falle von dreidimensionalen Simulationsrechnungen mit vergleichbarer Gitterauflösung (entsprechend 2.16 $\cdot 10^{8}$ Gitterzellen) ist dies schwerlich durchführbar. Die Aufgabe bei der numerischen Bestimmung von $Q_{\mathrm{R}}$ besteht darin, seine Abhängigkeit von der Gitterzellenweite $h$, der Ordnung des Strahlungstransportlösers und der Ordnung der auf einem Gitterelement vorliegenden hydrodynamischen Variablen zu quantifizieren und zu beurteilen. In der numerischen Berechnung, wurde die durch Gl. (4.28) dargestellte Form in atmosphärischen Bereichen welche durch eine mittlere freie Photonweglänge von $l \geq 10^{6} \mathrm{~cm}$ charakterisiert sind, benutzt. Wie von Bruls et al. (1999) ([4]) dokumentiert, zeigt dort die aus mathematischer Sicht äquivalente Form Gl. (4.27) aufgrund der Tatsache, daß die mittlere Intensität $J$ Gl. (2.44) und die Quellfunktion Gl. (4.6) praktisch gleich groß werden aufgrund von Auslöschungseffekten ein numerisch instabiles (oszillatorisches) Verhalten. In Bereichen mit $l<10^{6} \mathrm{~cm}$ hingegen wurde die Form Gl. (4.27) verwendet, da die Bestimmung der Divergenz des Strahlungsflusses Gl. (4.28) dann ungenau wird, weil es ebenfalls zu Auslöschungseffekten kommt.

Zur quantitativen Bestimmung der eingangs erwähnten Abhängigkeiten wurde $Q_{\mathrm{R}}$ für zwei, dem Anwendungsbereich verwandte zeitunabhängige Probleme berechnet. Zum einen war dies eine planparallele Schichtung ([83]). Zum anderen eine magnetische Flußschicht (Kap. 4.3). Obwohl für diese Probleme keine exakten 
Lösungen existieren, ist es in beiden Fällen doch möglich, mittels Konvergenzanalysen Aufschluß über die Güte der Resultate zu gewinnen. Dabei handelt es sich allerdings nicht um Konvergenzanalysen im strengen Sinn (Kap. 4.3). In beiden Fällen wurden die mit einer Gitterauflösung von $1 \mathrm{~km}$ und der $A 4$-Quadratur ermittelten Ergebnisse als Referenzlösungen betrachtet und die Konvergenz der gitterweitenabhängigen Lösung für $h \rightarrow 1 \mathrm{~km}$ untersucht. Im Falle der planparallelen Schichtung wurde dies von Bruls et al. (1999) ([4]) für die oben erwähnte Hybridmethode bereits ansatzweise gezeigt. Hier betrachten wir hauptsächlich den zweiten Anwendungsfall, bei dem Temperatur und Dichte am Rande der Flußschicht einen sehr scharfen Übergang aufweisen. Dieser wurde bewußt nicht über mehrere Gitterzellen verteilt, sondern geht innerhalb einer Gitterweite vonstatten. Auf diese weise läßt sich testen, wie stark der Einfluß von Gitterauflösung und Ordnung des Strahlungstransport-Lösers bei der numerischen Behandlung von Temperaturund Dichteinhomogenitäten (z.B. bei Schockwellen) ist und wie groß der damit verbundene effektive Strahlungsenergietransport und seine numerisch bedingten Ausschmierungen sind.

\subsubsection{Hydrodynamische Elementdaten}

Zur Berechnung von $I_{\nu}(\vec{r}, \vec{\mu})$ und $Q_{\mathrm{R}}$ auf jedem Gitterelement werden die Temperatur und die Dichte des Plasmas benötigt. Der benutzte 2D-MHD-Code kann entsprechend der geforderten Konvergenzordnung des Verfahrens, sowohl mit konstanten als auch mit linearen konservativen Elementdaten (Gl. 3.17) ([13]) arbeiten. Aus Rechenzeitgründen wäre es wünschenswert, wenn die Bestimmung von $Q_{\mathrm{R}}$ mit eben diesen Elementdaten durchgeführt werden könnte. Anhand des Problems der planparallelen Schichtung (Kap. 2.9.5) bei dem $Q_{\mathrm{R}}$ mit der $A 4-$ Quadratur berechnet wurde, werden die Abhängigkeiten von der Ordnung der hydrodynamischen Elementdaten und von der Rekonstruktionstechnik, mit der aus konstanten Elementdaten lineare ermittelt werden, erläutert. Abb. 4.17 (oben) zeigt für $i=0, \cdots, n$ alle vertikalen Schnitte $Q_{\mathrm{R}}\left(x_{i}, z\right)$ (mit $x_{0} \leq x_{i} \leq x_{n}, x_{0}, x_{n}$ : linker und rechter Rechengebietsrand) der mit linearen konservativen Elementdaten berechneten Referenzlösung in einer Darstellung. Abb. 4.17 (unten) zeigen analog zur oberen Darstellung alle vertikalen Schnitte von $Q_{\mathrm{R}}(x, z)$, welches mit konstanten konservativen Elementdaten für vertikale Auflösungen von $\Delta z=40 \mathrm{~km}$ (unten links) und $\Delta z=6 \mathrm{~km}$ (unten rechts) ermittelt wurde. Dabei wird deutlich, daß unabhängig von der vertikalen Auflösung die Berechnung von $Q_{\mathrm{R}}$ mit konstantem $T$ und $\rho$ pro Gitterelement fehlerhaft ist. 

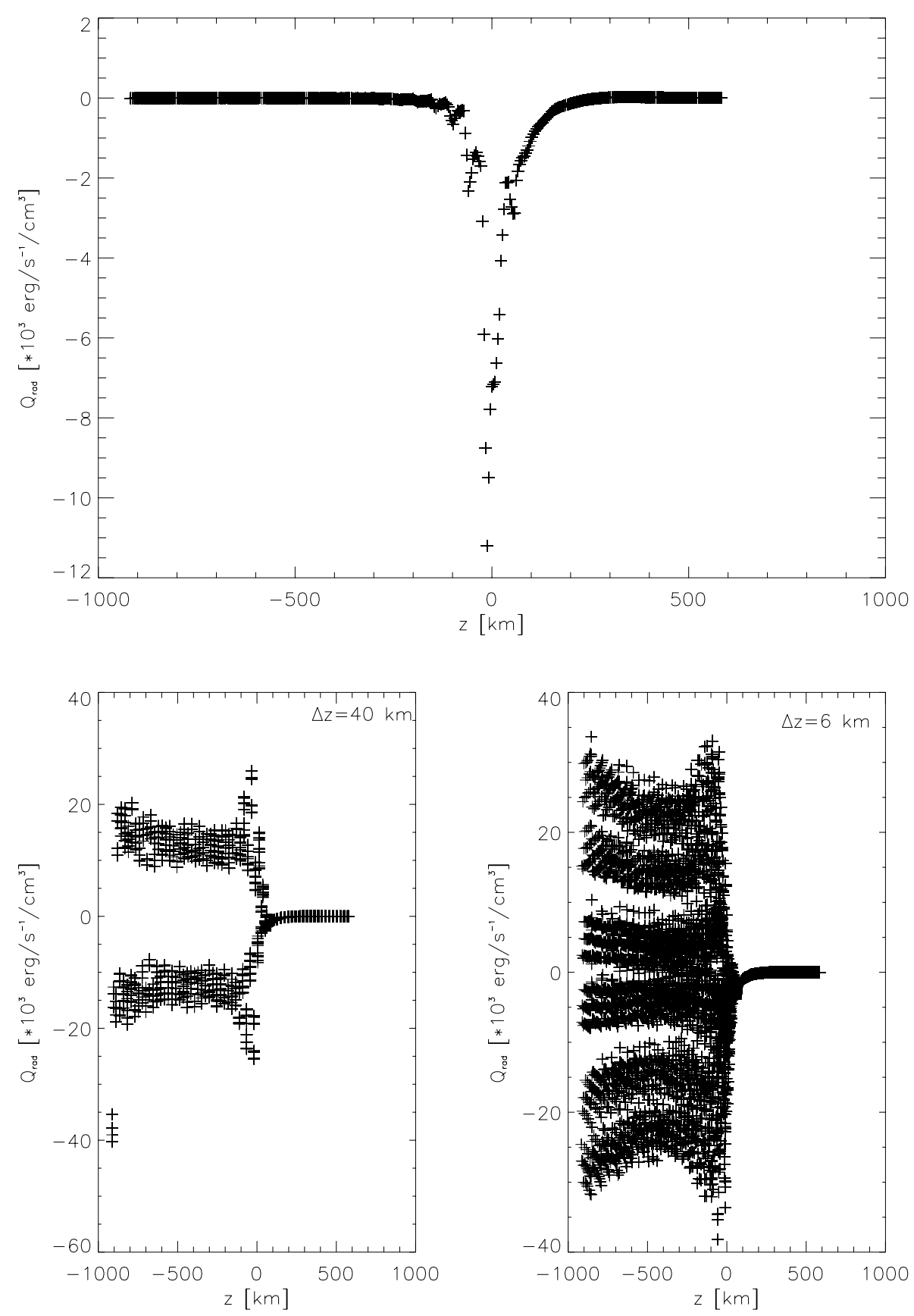

Abbildung 4.17: Gezeigt sind für $i=0, \cdots, n$ alle vertikalen Schnitte $Q_{\mathrm{R}}\left(x_{i}, z\right)$ (mit $x_{0} \leq x_{i} \leq x_{n} ; x_{0}, x_{n}$ : linker und rechter Rechengebietsrand) in einer Darstellung. Oben: Referenzlösung von $Q_{\mathrm{R}}$ berechnet für 65536 Gitterelemente $(\Delta z=10 \mathrm{~km})$ und linearen $T$ und $\rho$-Werten pro Gitterelement, die mittels Knotenrekonstruktion ([28], [29]) berechnet wurden. Unten: $Q_{\mathrm{R}}$ ermittelt mit konstantem $T$ und $\rho$ pro Gitterelement (links) für eine vertikale Auflösung von $\Delta z=40 \mathrm{~km}$ (rechts) für $\Delta z=6 \mathrm{~km}$. 
Dies läßt sich durch Betrachtung der formalen Lösung der Strahlungstransportgleichung verstehen:

$$
v_{i}(s)=v_{i}(0) e^{-\Delta \tau(0, s)}+\int_{0}^{s} S(\sigma) e^{-\Delta \tau(\sigma, s)} \mathrm{d} \sigma
$$

mit

$$
\Delta \tau(a, b):=\int_{a}^{b} \kappa(\sigma) \rho(\sigma) \mathrm{d} \sigma
$$

In den optisch dicken Bereichen $\left(\tau_{5000} \gg 1\right)$ ist der erste Teil von Gl. (4.29), welcher die Absorption beschreibt, vernachlässigbar. Mit konstantem $T$ und $\rho$ folgt pro Gitterzelle ein konstantes $S$ und ein konstantes $\kappa \rho$. Daraus resultieren unphysikalische Strahlungsflüsse (Abb. 4.17 oben), da $\kappa$ sehr sensibel auf die Temperatur- und Dichteänderungen reagiert und das Integral in Gl. (4.29) falsche Beiträge liefert. Zur Ermittelung eines für die ruhige Sonnenphotosphäre realistischen Strahlungsflußprofil reicht es daher nicht den Temperatur- und Dichteverlauf als stückweise konstante Funktionen auf das numerische Gitter zu interpolieren. Da bei einer vertikalen Auflösung von $h=6 \mathrm{~km}$ im Modell der planparallelen Schichtung sowohl Temperatur als auch Dichte als näherungsweise linear betrachtet werden können, erhält man durch stückweise lineare Interpolation von $T$ und $\rho$ auf jedes Gitterelement im Falle der planparallelen Schichtung ein realistischeres Strahlungsflußprofil (Abb. 4.18).

Abb. 4.18 (oben) zeigt das $Q_{\mathrm{R}}$, welches mit vom 2-D-MHD-Code gelieferten, rekonstruierten, linearen Elementdaten berechnet wurde ([21], [30]). Auch in diesem Fall sind die, für die optisch dicken Regionen ermittelten Werte unakzeptabel fehlerhaft, was damit zu begründen ist, daß in diesem Fall zwar ein lineares Verhalten von Temperatur und Dichte pro Gitterelement vorlag, allerdings kein stetiger Übergang zwischen benachbarten Elementen bestand, so daß es auch in diesem Fall zur Verletzung der Erhaltungseigenschaft des Strahlungsflusses kommt.

Die besten Resultate dargestellt in Abb. 4.18 (unten) sind mit linearen Elementdaten erzielt worden, welche durch Knotenrekonstruktion ermittelt wurden ([28], [29]). Dabei sind die linearen Profile von $T$ und $\rho$ auf die Elementschwerpunkte interpoliert worden. Aus den Schwerpunktswerten aller an einem Gitterknoten $i$ partizipierende Gitterelemente werden die flächengewichteten Mittelwerte $\overline{T_{i}}, \overline{\rho_{i}}$ für den Gitterknoten $i$ berechnet. Dies wird für alle Knoten des Rechengitters durchgeführt. Aus den 3 Gitterknoten, welche ein Gitterelement aufspannen, werden anschließend die linearen Flächenfunktionen für $T$ und $\rho$ ermittelt.

Die mit dieser Technik rekonstruierten Elementdaten haben den Vorteil, daß sie stetig an den Elementgrenzen sind und somit die Erhaltung des Strahlungsflusses gewahrt ist. 

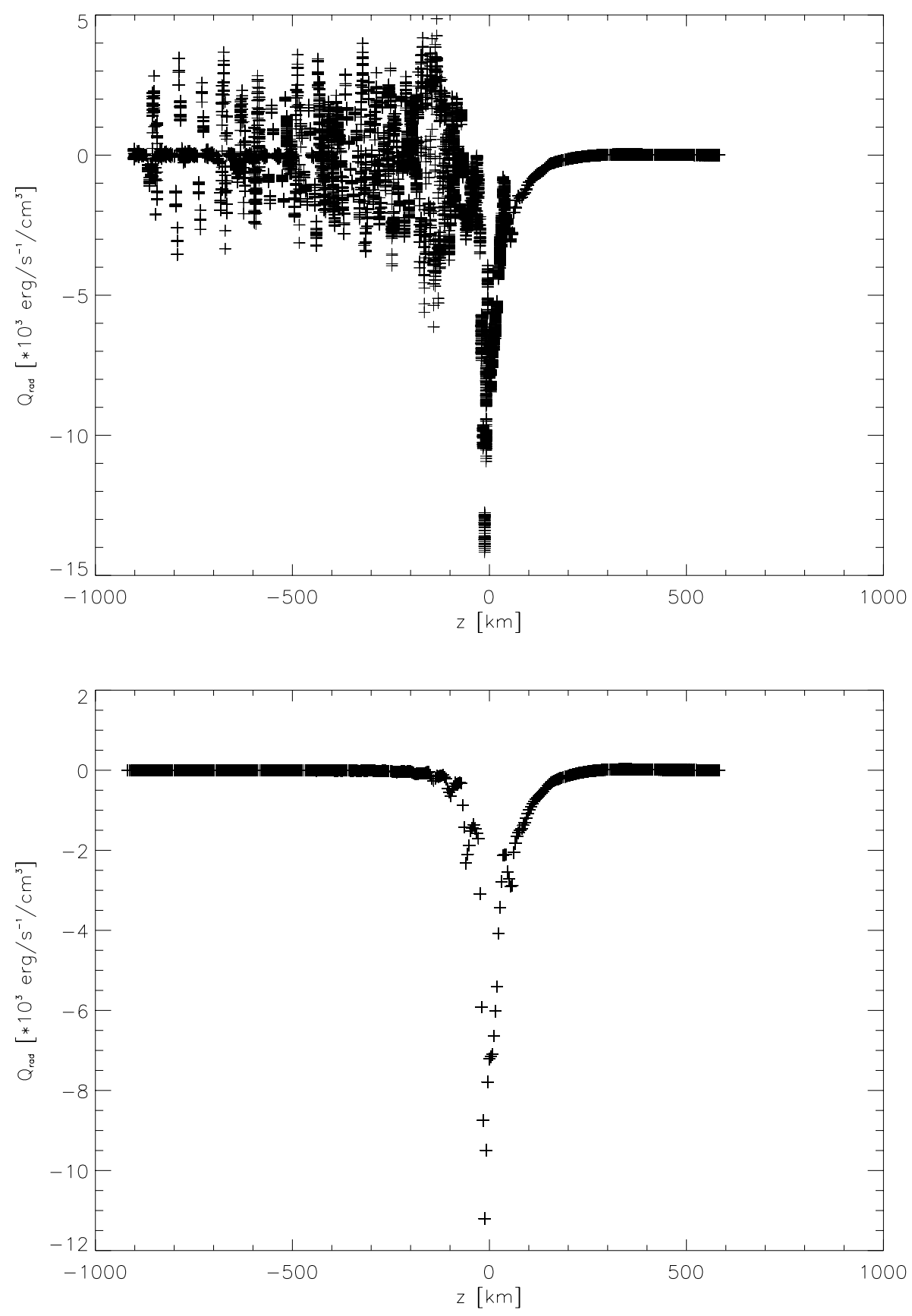

Abbildung 4.18: Darstellung zeigt für $i=0, \cdots, n$ alle vertikalen Schnitte $Q_{\mathrm{R}}\left(x_{i}, z\right)$ (mit $x_{0} \leq x_{i} \leq x_{n}, x_{0}, x_{n}$ : linker und rechter Rechengebietsrand) auf einmal. Oben: $Q_{\mathrm{R}}$ berechnet für 65536 Gitterelemente $(\Delta z=10 \mathrm{~km})$ und linearen $T$ - und $\rho$-Werten pro Gitterelement, die mittels Knotenrekonstruktion ([28], [29]) berechnet wurden. Unten: $Q_{k}$ berechnet für 65536 Gitterelemente $(\Delta z=10 \mathrm{~km})$ und linearem $T$ und $\rho$ pro Gitterelement, die vom MHD-Löser geliefert wurden ([21], [30]). 


\subsubsection{Ordnung des Strahlungstransportlösers}

Die Strahlungstransportgleichung wurde mit den neuentwickelten ESC1(IRK)- und dem ESC2-mod-Verfahren (Kap. 4.2.1) für das Problem der magnetischen Flußschicht (Kap. 4.3) gelöst, um den Einfluß der Ordnung des Strahlungstransportlösers zu ermitteln. Die diskrete frequenzunabhängige Form von Gl. (4.28) für den Strahlungsquellterm stellt sich folgendermaßen dar:

$$
Q_{\mathrm{R}}^{j}=\int_{T_{j}} \nabla \cdot \vec{F}^{r a d} d A=\int_{\partial T_{j}} \vec{F}^{r a d} \cdot \vec{n} d s
$$

Hier wurde zur $Q_{\mathrm{R}}^{j}-$ Berechnung die zweite Form verwendet:

$$
Q_{\mathrm{R}}^{j}=\int_{\partial T_{j}} \vec{F}^{r a d} \cdot \vec{n} d s
$$

welche im folgenden für die ESC1- und die ESC2-Methode erläutert wird. Der Strahlungsfluß wird nach Gl. (2.45) berechnet.

Im weiteren wird rad aus Gründen der Übersichtlichkeit weggelassen. Im Fall des ESC1-Verfahren ist die Intensität und somit auch $\vec{F}$ an den Knoten bekannt. Um ein Verhalten 1. Ordnung für $Q_{\mathrm{R}}$ auf einem Dreieckselement $T_{j}$ zu erhalten, muß $\vec{F} \cdot \vec{n}$ an den Kantenmittelpunkten berechnet werden. Aus diesem Grund wird $\vec{F}$ an den Kantenmittelpunkten durch lineare Interpolation der Knotenwerte von $\vec{F}$ ermittelt (Abb. 4.19):

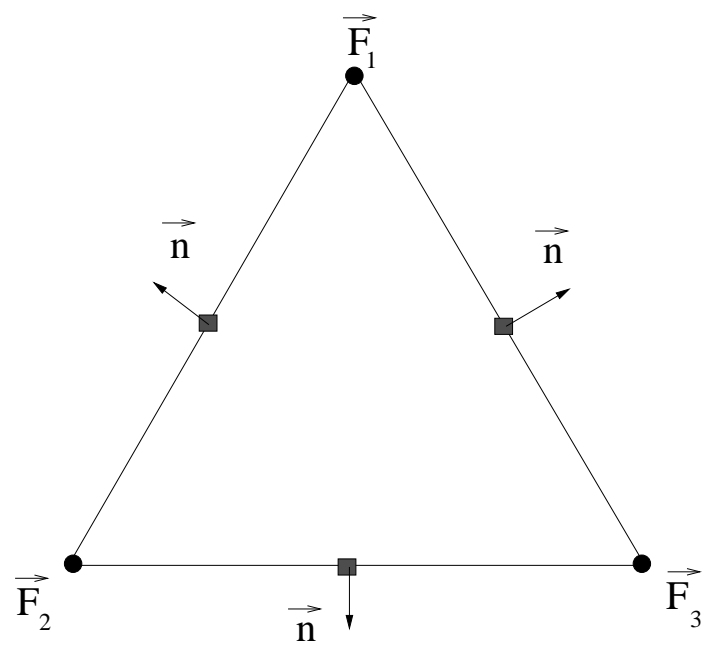

Abbildung 4.19: Zur Bestimmung von $Q_{\mathrm{R}}^{j}$ beim ESC1-Verfahren. Die Punkte kennzeichnen die Orte an denen Intensitäten und der Strahlungsfluß Gl. (2.45) bekannt sind. An den durch Quadrate gekennzeichneten Orten werden aus den Knotenwerten von $\vec{F}_{i}$ Kantenmittelpunktswerte interpoliert und mit der Oberflächennormalen multipliziert.

$$
Q_{\mathrm{R}}^{j}=\sum_{l=1}^{3} \frac{h}{2}\left(\vec{F}_{l}+\vec{F}_{l+1}\right) \cdot \vec{n}
$$


$\left(\vec{F}_{4}=\vec{F}_{1}\right)$. Im Fall des ESC2-Verfahrens ist die Intensität und damit auch $\vec{F}$ an den durch ein Kreis gekennzeichneten Punkten bekannt und man erhält ein Verhalten 2. Ordnung für $Q_{\mathrm{R}}$, indem $\int_{\partial T_{j}} \vec{F} \cdot \vec{n} d s$ mittels der Simpsonschen-3-PunktQuadraturformel numerisch integriert wird:

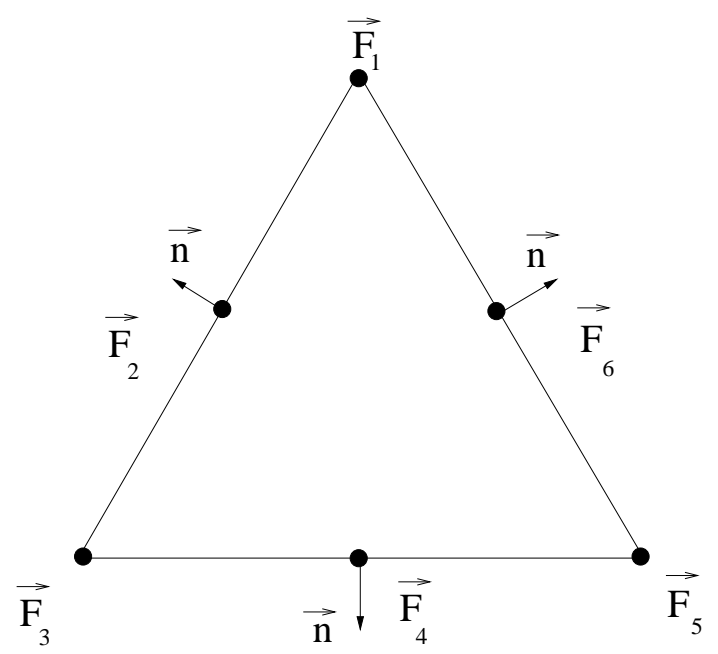

Abbildung 4.20: Dreieck $T_{j}$. Die Punkte kennzeichnen die Orte an denen mittels ESC2Verfahren die Koeffizienten Gl. (4.12) berechnet wurden und somit die Intensitäten und der Strahlungsfluß Gl. (2.45) bekannt sind $\left(\vec{F}_{i}, \quad i=1, \ldots, 5\right)$. Numerische Integration von $\vec{F} \cdot \vec{n}$ entlang einer Dreiecksseite mittels der Simpsonschen-3-Punkt-Quadraturformel und anschließender Aufsummation liefert das Resultat für das Integral in Gl. (4.31).

$$
Q_{\mathrm{R}}^{j}=\sum_{l=1}^{5} \frac{h}{2} \cdot\left(\frac{1}{3} \vec{F}_{l}+\frac{4}{3} \vec{F}_{l+1}+\frac{1}{3} \vec{F}_{l+2}\right) \cdot \vec{n}
$$

$\left(\vec{F}_{7}=\vec{F}_{1}\right)$, (Knotengewichte $: \frac{1}{3}$, Kantenmittelpunktsgewicht: $\left.\frac{4}{3}\right)$.

Während sich beim planparallelen Modell kein merklicher Unterschied zwischen erster und zweiter Ordnung zeigte (Abb. 4.21), trat er in sehr viel stärkerem Maß beim Problem der magnetischen Flußschicht auf. Abb. 4.22 zeigt in einer Farbabbildung das generelle Verhalten von $Q_{\mathrm{R}}$ im Falle der Flußschicht. In dem Tiefenbereich von $-400 \mathrm{~km}-+200 \mathrm{~km}$ strahlt die vermeintlich heißere Umgebung in die Flußschicht, Energie wird per Strahlung ins Flußschichtinnere transportiert, wodurch diese geheizt und die Umgebung gekühlt wird.

Im Prinzip sollte mit der Berechnung 2.Ordnung des Strahlungsquellterms dieselbe Genauigkeit erzielbar sein wie mit der Berechnung 1.Ordnung, jedoch auf einem gröberen Gitter und somit in kürzerer Rechenzeit. Konkret bedeutet das, daß auf einem von dem MHD-Löser vorgegebenen Rechengitter die räumliche Auflösung der Heizungs- und Kühlungszonen an den Flußschichträndern bei gleicher Rechenzeit im Fall der Berechnung 2.Ordnung deutlich besser sein sollte. Da in diesem Fall keine Referenzlösung existiert, wurde die Güte der Resultate anhand einer angenährten Konvergenzbetrachtung quantifiziert.

Dazu wurden innerhalb zweier räumlicher Zonen (Abb. 4.23), die $L_{1}$-Normen von 

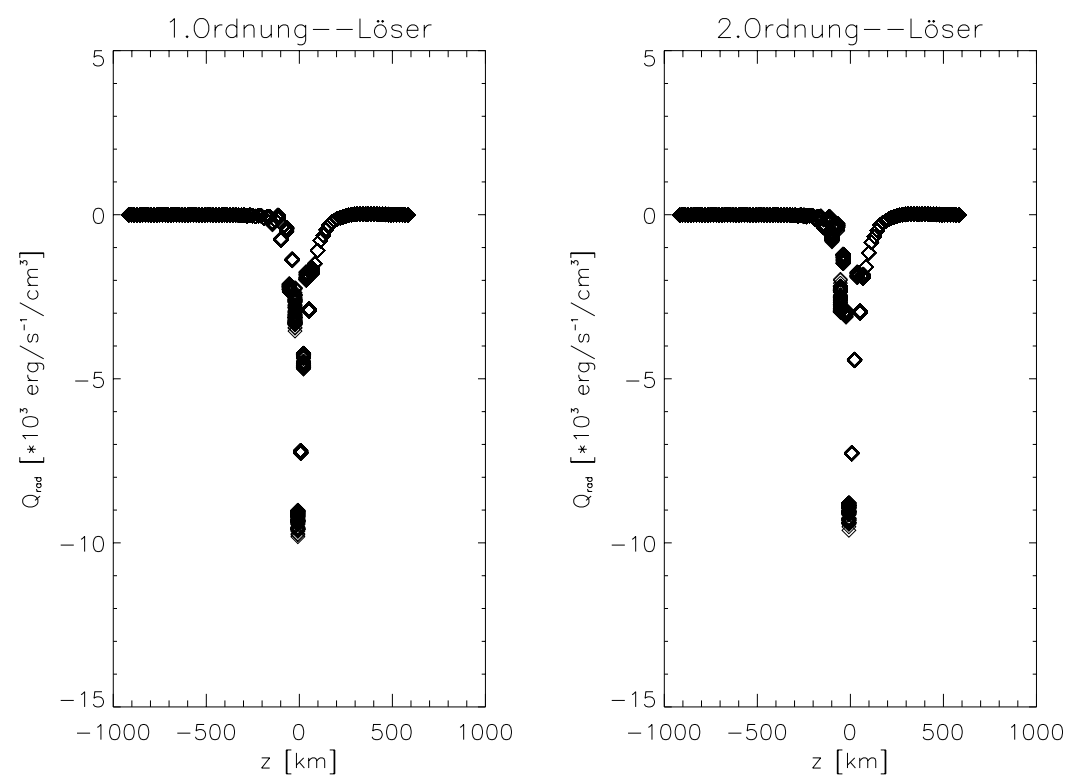

Abbildung 4.21: Die Darstellung zeigt für $i=0, \cdots, n$ alle vertikalen $S c h n i t t e ~ Q_{\mathrm{R}}\left(x_{i}, z\right)$ (mit $x_{0} \leq x_{i} \leq x_{n}, x_{0}, x_{n}$ : linker und rechter Rechengebietsrand) auf einmal. Links: $Q_{\mathrm{R}}$ für die planparallele Schichtung mit der ESC1-Methode und einer Gitterauflösung von $\Delta z=6 \mathrm{~km}$ berechnet. Rechts: Berechnung ESC2-mod-Methode bei einer Gitterauflösung von $\Delta z=6 \mathrm{~km}$ durchgeführt.

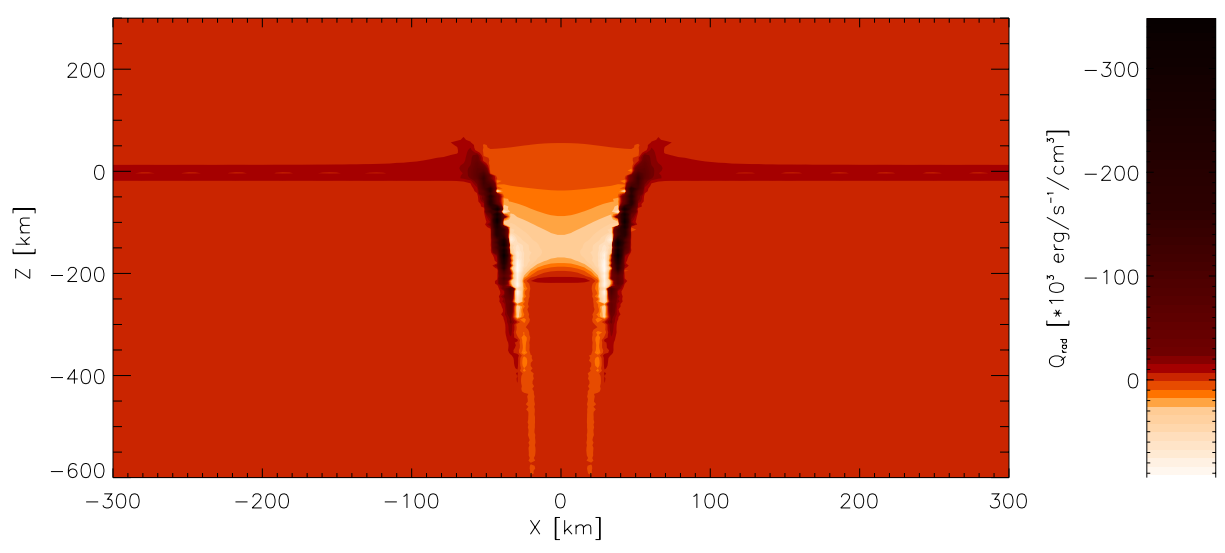

Abbildung 4.22: $Q_{\mathrm{R}}$ für das Modell der magnetischen Flußschicht, berechnet mit dem ESC1-Verfahren. Die Wechselwirkung zwischen Flußschichtinnerem und Flußschichtäusserem ist auf den Bereich zwischen $z=-400 \mathrm{~km}$ und $z=0 \mathrm{~km}$ beschränkt.

$Q_{\mathrm{R}}^{i}$ und $Q_{\mathrm{R}}^{e}$ für Gitterweiten von 40, 26, 13, 10, 6, 2 und $1 \mathrm{~km}$ berechnet:

$$
\begin{aligned}
\left\|Q_{\mathrm{R}}^{i}\right\| & =\int_{0}^{R_{i}}\left|Q_{\mathrm{R}}^{i}\left(x_{i}, z\right)\right| d x d z=\sum_{j=1, \cdots, n_{\text {max }}^{i}}\left|A_{j} Q_{\mathrm{R}, \mathrm{j}}^{i}\right|, \\
\left\|Q_{\mathrm{R}}^{e}\right\| & =\int_{0}^{R_{e}}\left|Q_{\mathrm{R}}^{e}\left(x_{e}, z\right)\right| d x d z=\sum_{j=1, \cdots, n_{\max }^{e}}\left|A_{j} Q_{\mathrm{R}, \mathrm{j}}^{e}\right|
\end{aligned}
$$


( $A:=$ Flächeninhalt des Gitterelements, $n_{\max }^{i}, n_{\max }^{e}$ : Gesamtzahl der Gitterelemente, die in ausgewählten Zonen liegen).

Die horizontalen Ausdehnungen der Zonen $R_{e}, R_{i}$ sind für $R_{e}$ mit $50 \mathrm{~km}$ höhenunabhängig und $R_{i}$ entsprechend der höhenabhängigen Schichtdicke $d$ gewählt worden (Abb. 4.23). $R_{i}$ mußte höhenabhängig gewählt werden, da im Inneren die mittlere freie Weglänge der Photonen $l_{i}=\left[\kappa_{i} \rho_{i}\right]^{-1}$ mit der geometrischen Höhe $z$ stärker zunimmt als im äußeren Breich und die von aßerhalb der Flußschicht kommende Strahlung über eine größere Wegstrecke das Flußschichtinnere heizen kann, da dies optisch dünner ist. Für $R_{e}$ war die Annahme eines höhenunabhängigen Streifens mit $50 \mathrm{~km}$ Breite außerhalb der Flußschicht ausreichend. Eine Vergrößerung veränderte das Resultat nicht wesentlich, wohingegen für $R_{e} \ll l_{e}$ ( $l_{e}$ mittlere freie Weglänge außerhalb der Flußschicht $l_{e}=\left[\kappa_{e} \rho_{e}\right]^{-1}$ ) die Resultate falsch werden mußten, da die Integration nicht mehr über den ganzen gekühlten Bereich ausgeführt wurde.

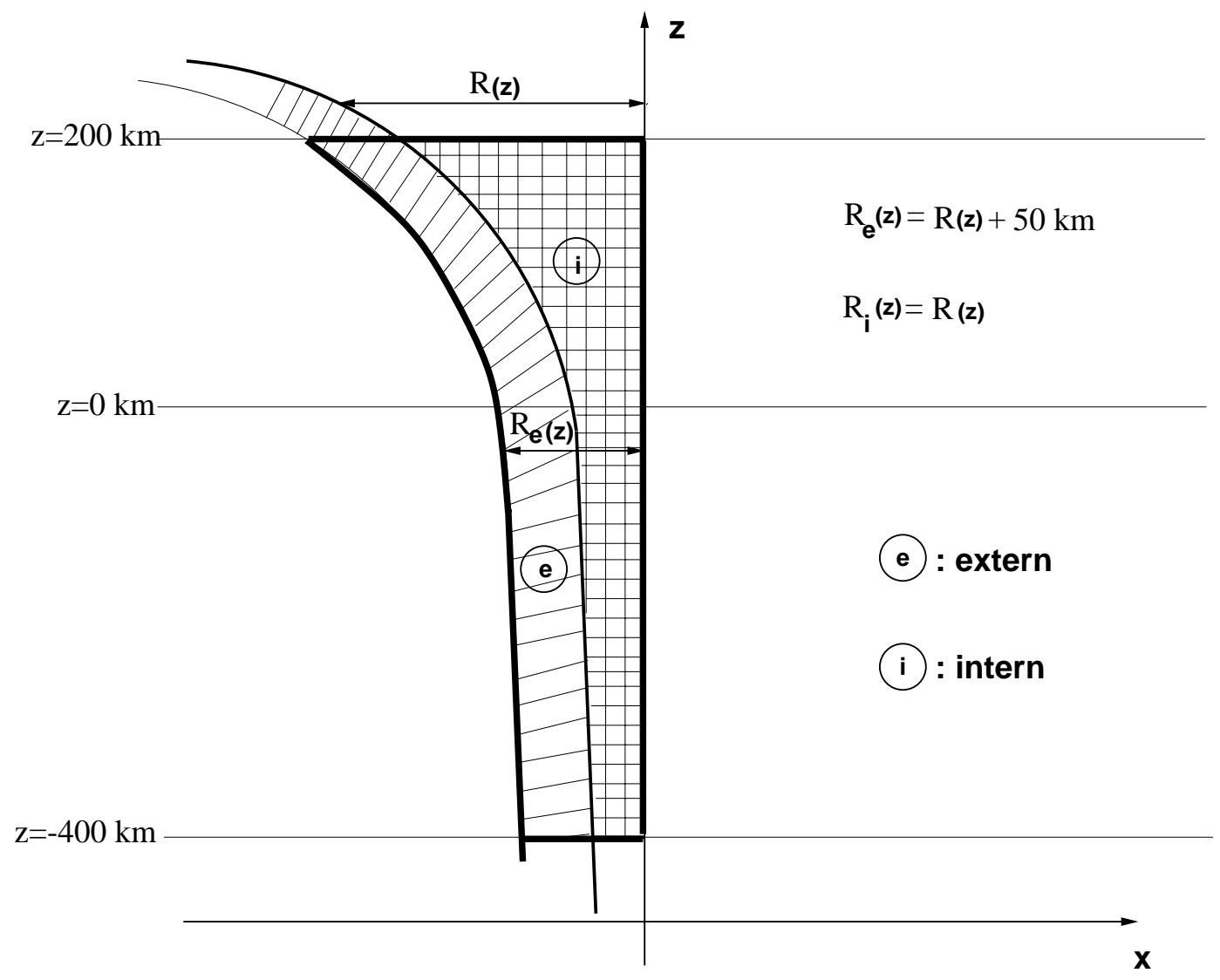

Abbildung 4.23: Schematische Darstellung der einen Hälfte der magnetischen Flußschicht mit beiden Zonen. Die innerhalb der Flußschicht liegende Zone, in der die $L_{1}-$ Norm von $Q_{\mathrm{R}}^{i}$ berechnet wurde, ist durch das Netz gekennzeichnet. Die außerhalb der Flußschicht angebrachte horizontal mit $50 \mathrm{~km}$ ausgedehnte Bereichzone, in $\operatorname{der} Q_{R}$ berechnet wird, ist durch Streifen markiert. 

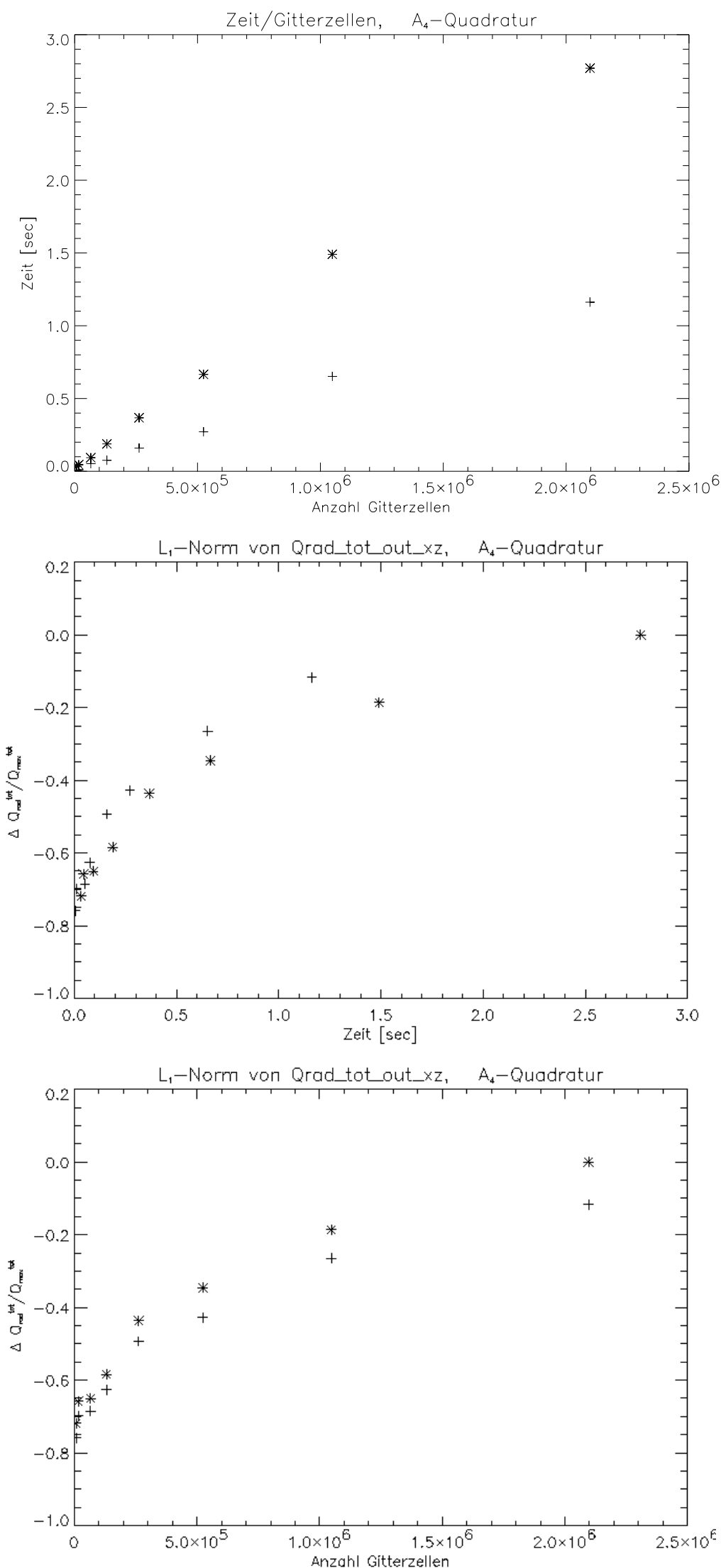

Abbildung 4.24: Abweichungen der auflösungsabhängigen $L_{1}$-Fehler. Die Referenzlösung für das externe Gebiet wurde mit $2 \cdot 10^{6}$ Gitterzellen ( $1 \mathrm{~km}$ Gitterweite) und 12 Strahlungsrichtungen ermittelt. Oben: Rechenzeit gegen Gitterzellenanzahl. Mitte: Abweichungen gegen Rechenzeit. Unten: Abweichungen gegen Fehler. Die Kreuze repräsentieren die Resultate 1. Ordnung und die Sterne die Resultate 2. Ordnung. 

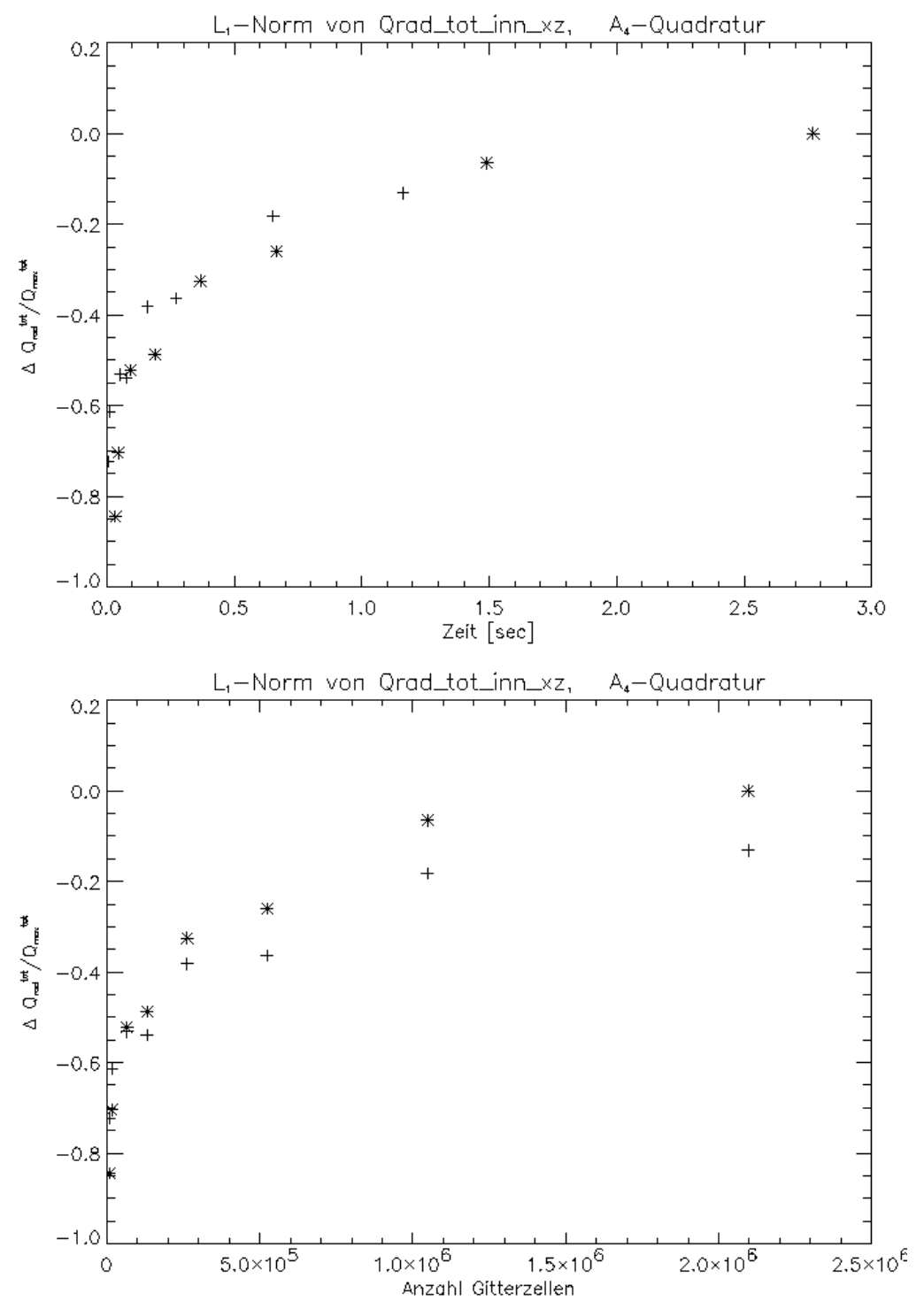

Abbildung 4.25: Abweichungen der auflösungsabhängigen $L_{1}-$ Fehler. Die Referenzlösung für das interne Gebiet wurde mit $2 \cdot 10^{6}$ Gitterzellen $(1 \mathrm{~km}$ Gitterweite) und 12 Strahlungsrichtungen ermittelt. Oben: Abweichungen gegen Rechenzeit. Unten: Abweichungen gegen Fehler aufgetragen. Die Kreuze repräsentieren die Resultate 1. Ordnung und die Sterne die Resultate 2. Ordnung.

Zwar benötigt die Quelltermberechnung 2. Ordnung für jede untersuchte Gitterauflösung etwa die doppelte Berechnungszeit (Abb. 4.24 Mitte), doch Abb. 4.24 (unten) und Abb. 4.25 (unten) verdeutlichen, daß selbst bei $2 \cdot 10^{6}$ Gitterzellen die mit der Methode 1.Ordnung ermittelten Resultate mehr als $10 \%$ Abweichung zu den mit der Methode 2.Ordnung erzielten Resultaten aufweisen. Dies gilt sowohl für den externen, als auch den internen Bereich (Abb. 4.23). Die mit der Methode 2. Ordnung und $2 \cdot 10^{6}$ Gitterzellen erzielten Resultatestellen die Referenzlösung dar. Dies gründet sich darauf, daß, wie in Kap. 4.3 gezeigt, der ESC2-mod-Löser wesentlich genauer als der ESC1-Löser ist und die in Abb. 4.20 dargestellte und in G1. (4.33) formulierte Art und Weise, $Q_{\mathrm{R}}$ zu berechnen, zum ESC2-Löser konsistent ist. 


\subsubsection{Ausblick bei der numerischen $Q_{\mathrm{R}}$-Bestimmung}

Wie stark sich die Abhängigkeit der $Q_{\mathrm{R}}$-Resultate von der Ordnung der Methoden in einer zeitabhängigen Simulation nun wirklich auswirkt, kann nur durch einen Vergleich von MHD-Simulationsergebnissen, welche einmal mit der Methode 1. Ordnung und einmal mit der Methode 2. Ordnung ermittelt werden, bestimmt werden. Desweiteren müssen noch systematische Untersuchungen bezüglich der Abhängigkeit der $Q_{\mathrm{R}}$-Resultate von der Winkelquadratur durchgeführt werden.

Die Nutzung einer Winkelquadratur mit 12 unterschiedlichen Winkelrichtungen ist aus Rechenzeitgründen in zweidimensionalen Simulationen vertretbar gerade noch. In dreidimensionalen Rechnung wird dies kaum durchführbar sein. Es gibt erste Vorüberlegungen und Tests zum Einsatz von Quadraturformeln mit $n$ verschiedenen Winkelrichtungen (z.B. 12, 24, 48 etc.), allerdings mit dem Unterschied, daß in jedem Zeitschritt die Strahlungstransportgleichung nur für eine bestimmte Anzahl $m$ Richtungen (mit $m=\frac{n}{a}$ ) gelöst wird und die spezifische Intensität $I^{m}$ berechnet wird. Für die in einem Zeitschritt nicht neu berechneten Intensitäten $I^{n-m}$ der nicht abgearbeiteten Winkelrichtungen werden die in den vorherigen Zeitschritten berechneten, wiederverwendet. Dadurch kann die zur $Q_{\mathrm{R}}$-Bestimmung notwendige Winkelintegration durchgeführt werden und die pro Zeitschritt sehr aufwändige Bestimmung von $I^{n}$ verringert sich auf den $a$-ten Teil des Aufwandes, der zur Berechnung von $n$ Richtungen pro Zeitschritt nötig wäre. Dabei gilt es mittels genauerer Untersuchungen den Teiler $a$ zu bestimmen. Einer der Ansatzpunkte ist dabei die Zeitskala, auf der sich die hydrodynamischen Variablen, ändern. Ändert sich dieser 'hydrodynamische Hintergrund' beispielsweise über $n$ Zeitschritte nur unwesentlich, so könnte es ausreichend sein, in jedem Zeitschritt die Intensität einer Richtung $I^{1}$ neu zu berechnen und anstelle der nichtberechneten die in den vorherigen Zeitschritten berechneten Intensitäten wiederzuverwenden. Ein möglicher Indikator dazu wurde bislang nicht entwickelt, sollte aber von einer Änderung der mittleren freien Photonweglänge $l=[\kappa \rho]^{-1}$ pro Gitterzelle abhängen.

Auch wäre es denkbar, eine Hybridmethode bestehend aus den Methoden 1. und 2. Ordnung einzusetzen. Überlegungen wurden dazu von Dedner \& Vollmöller (2001) ([14]) angestellt. Sie zeigen anhand eines vergleichbaren Problems, daß die eigentliche Stärke der ESC2-Methode wie in Abb. 4.26 gezeigt in dem optisch dünnen Atmosphärenbereich liegt, in dem Bereich also, in dem kaum noch Absorption stattfindet. Dies könnte bedeuten, daß die Unterschiede in den Resultaten von 1. und 2. Ordnung im Falle von steilen Gradienten in $T$ und $\rho$ in optisch dünnen Bereichen noch sehr viel deutlicher werden. Als anschließendes Testproblem soll $Q_{\mathrm{R}}$ für einen sich ausbreitenden Schock untersucht werden. Denn für einen in einem gravitativ geschichteten Medium propagierenden Schock ist die thermische Energiedissipation von großer Bedeutung und entscheidet darüber, wie weit er in die oberen atmosphärischen Schichten kommen kann. Benutzt man im Falle numerischer Simulationen einen 'ausschmierenden' Strahlungstransportlöser (z.b. ESC1), wie in Abb. 4.15 dargestellt, so wird dieser dafür sorgen, daß die Schockfront thermisch ausgeschmiert wird und 'nicht weit kommt'. Ein nicht 'ausschmierender' Löser (ESC2) hingegen erhält dieses hinter der Schockfront existierende Depot an thermischer Energie aufrecht. Solange es existiert, kann der Schock als solcher weiterwandern. 


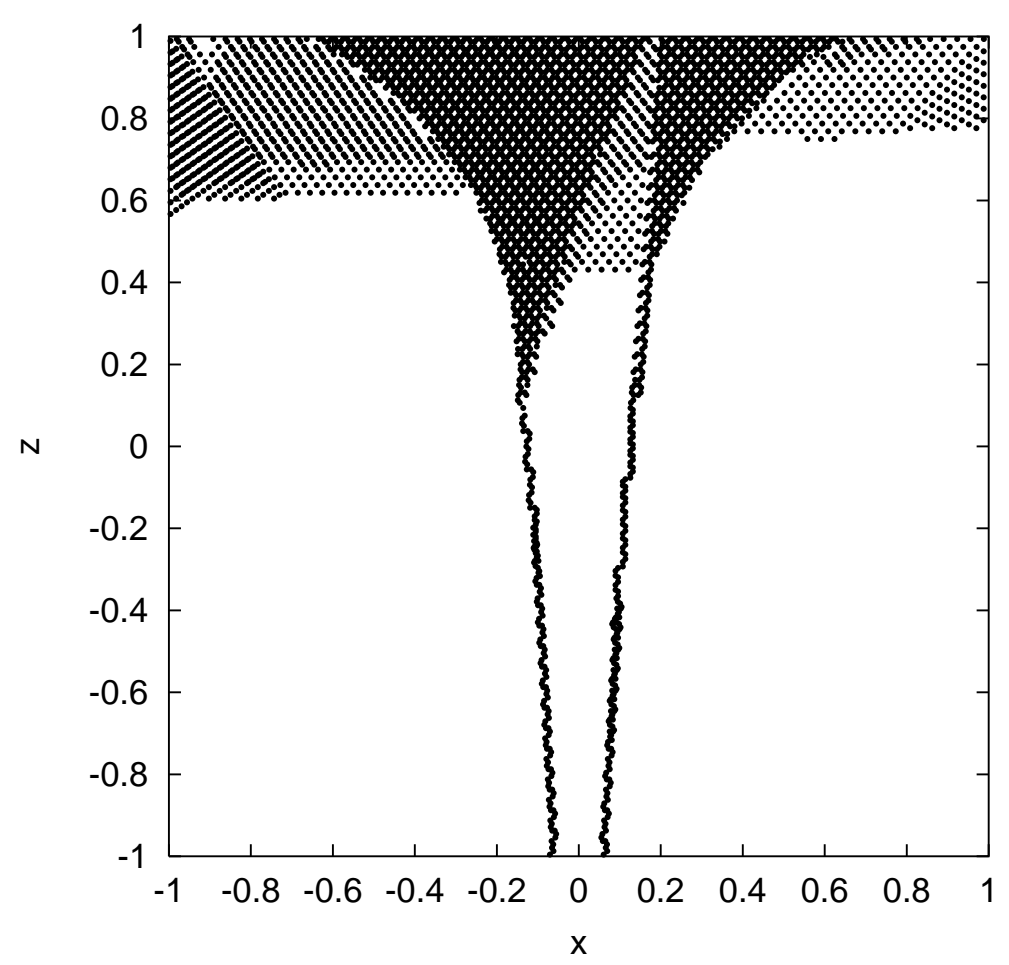

Abbildung 4.26: Markierung der Bereiche in denen die magnetische Flußschicht mit dem ESC1- (weiß) und mit dem ESC2-Löser (dunkel) berechnet wird. Als Indikator diente ein Residuenschätzer ([14]). 


\section{Kapitel 5}

\section{Magnetfeldverstärkung durch Konvektion}

\subsection{Hintergrund, bisherige Rechnungen}

Außerhalb von Sonnenflecken ist der überwiegende Teil des magnetische Flusses in der solaren Photosphäre in magnetischen Flußröhren konzentriert. Im sogenannten flux-expulsion-Prozeß ([69]) konzentrieren konvektive Strömungen den magnetischen Fluß in den Abstromgebieten zwischen den Granulen. Dieser Prozeß kann für die in der unteren Sonnenphotosphäre typischen Werte von Massendichte und horizontaler Strömungsgeschwindigkeit von $\rho=3 \times 10^{-7} \mathrm{~g} / \mathrm{cm}^{3}$ und $v_{x}=2.5$ km/s nur Flußröhren mit einer Magnetfeldstärke von der Größenordnung der Äquipartitionsfeldstärke $B=\sqrt{4 \pi \rho v_{x}^{2}}$ von $0.5 \mathrm{kG}$ erzeugen, nicht aber die beobachteten Werte von 1.5 kG. Zur weiteren Magnetfeldverstärkung kann es durch eine abwärtsgerichtete Strömung entlang der Feldlinien kommen, die eine partielle Evakuierung der Magnetfeldkonzentrationen bewirkt. Diese hat ihre Ursache in der Zunahme des magnetischen Drucks, welcher die horizontalen Strömungen, die heißere Materie aus der Umgebung der Magnetfeldkonzentrationen zu diesen hin transportieren, so stark abschwächt, daß die radiative Energieabstrahlung und die damit verbundene Abkühlung des Flußröhreninneren durch konvektiven Energietransport nicht mehr ausgeglichen werden kann. Aufgrund der andauernden radiativen Abkühlung und der starken Superadiabatizität unterhalb der Photosphäre kommt es entlang der Feldlinien zu einer beschleunigten Abströmung der abgekühlten Materie und somit zur Evakuierung der oberen Schichten. Zur Magnetfeldverstärkung und Ausbildung von Magnetfeldkonzentrationen im Kilogaußbereich kommt es dabei, weil sich wegen des abnehmenden Gasdrucks innerhalb der Magnetfeldkonzentrationen das Druckgleichgewicht mit der Umgebung nur einstellen kann, indem der magnetische Druck entsprechend zunimmt. Dieser Prozeß wird auch als konvektiver Kollaps bezeichnet und ist für dünne Flußröhren von Spruit (1978), Parker (1978), Webb $\&$ Roberts (1978) und anderen anhand eines linearen Ansatzes und von Venkata- 
krishnan $(1983,1985)$ und Hasan (1985) mittels numerischer Simulationen studiert worden ([85], [61], [73], [101], [102], [38]). Grossmann-Doerth et al. (1998) führten auch numerische Simulationen durch, wobei sie das vollständige System der MHD-Gleichungen lösten. Auch Beobachtungen lassen sich im Sinne dieses Prozesses interpretieren ([108], [55]). Spruit (1978) fand mittels linearer Stabilitätsanalyse, daß die typische Zeitskala, innerhalb der der konvektive Kollaps abläuft, der hydrodynamischen Zeitskala entspricht. Hasan (1985) studierte den Prozeß unter dem Einfluß eines horizontalen radiativen Energieaustausch durch Berechnung der zeitabhängigen nichtlinearen MHD-Gleichungen in der Näherung dünner Flußröhren. Er fand eine überstabile Oszillation mit einer Frequenz von $10^{3}$ s, welche die Folge der radiativen Heizung der durch den konvektiven Kollaps evakuierten Flußröhre ist. Grossmann-Doerth et al. (1998) ([36]) simulierten 5 Minuten einer sich ausbildenden magnetischen Flußschicht in einem über $1400 \times 2400 \mathrm{~km}^{2}$ ausgedehnten photosphärischen Bereich. Sie fanden, daß der Verstärkungsprozeß innerhalb von ca. 100s abläuft. Die Stärke der Evakuierung, die maximale Feldstärke und die Lebensdauer der Magnetfeldkonzentrationen hängen stark von dem im gesamten Rechengebiet enthaltenem magnetischen Fluß ab. Im Fall eines anfänglich homogenen vertikalen Magnetfelds von 100 Gauß erreicht die Feldstärke nach etwa 150s ihr Maximum und das Zentrum der Flußkonzentration wird sehr hell, wobei der Kontrast der Kontinuumsintensität 150\% erreicht. Die sich daran anschließende Abnahme der Magnetfeldstärke wird von einer Abnahme des Kontrasts begleitet und ist durch eine Aufströmung, die sich in der oberen Photosphäre zu einem Schock aufsteilt, bedingt.

\subsection{Simulationsresultate}

Im Rahmen dieser Arbeit wurde die Ausbildung von Magnetfeldkonzentrationen in der solaren Photosphäre mit einem adaptiv arbeitenden 2D-MHD-Code der in Kap. 2.8 beschriebenen und im weiteren als WDV-Code bezeichnet ist, numerisch simuliert. Das Simulationsgebiet erstreckte sich horizontal über $1.2 \cdot 10^{4} \mathrm{~km}$ und vertikal zwischen $300 \mathrm{~km}$ über und $900 \mathrm{~km}$ unter $\tau_{5000}=1$ und war damit 5 mal breiter als das mit dem Grossmann-Doerth et al. (1998) ([36]) gearbeitet haben. Es wurden 60 min solarer Magnetokonvektion simuliert. Die minimale und die maximale Gitterweite betrugen $10 \mathrm{~km}$ bzw. $67 \mathrm{~km}$. Es wurde die zeitliche Entwicklung eines anfangs homogenen vertikalen Magnetfelds der Stärke 100 Gauß, welches in eine vollentwickelte solare Konvektion eingebracht wurde, untersucht.

In den Abbildungen 5.1, die die Magnetfeldlinien und die Temperaturisoflächen im gesamten Rechengebiet zu 15 verschiedenen Zeitpunkten zeigen, ist in den ersten 9 Abbildungen sowohl der Flux-expulsion-Prozeß als auch der konvektive Kollaps erkennbar. Die letzten 6 Abbildungen stellen die Wechselwirkung zwischen Magnetfeld und der solaren Granulation zu späteren Zeitpunkten dar. Das jeweils zugehörige Geschwindigkeitsfeld ist in den Abbildungen 5.2 gezeigt. 


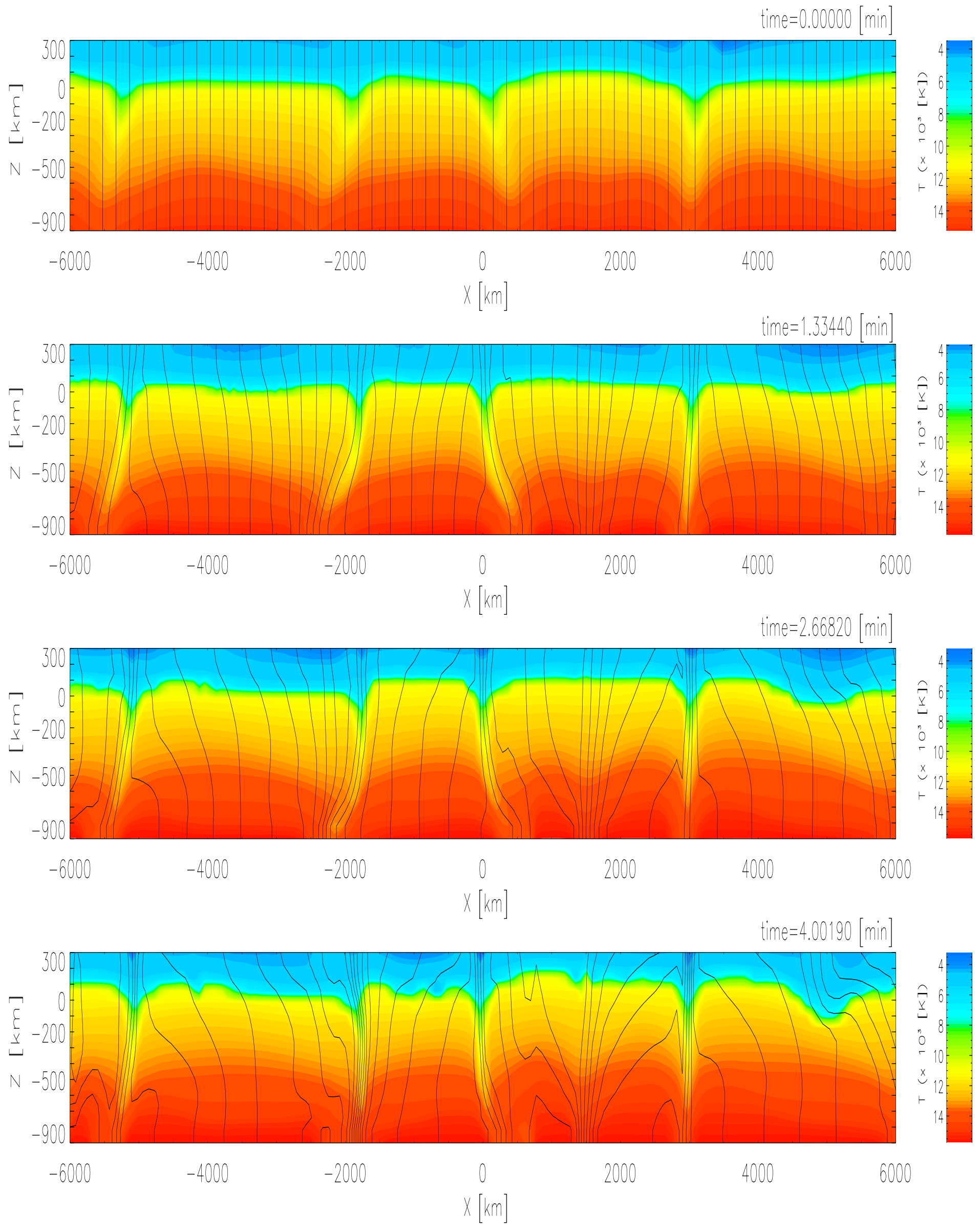




\section{KAPITEL 5. MAGNETFELDVERSTÄRKUNG DURCH KONVEKTION}
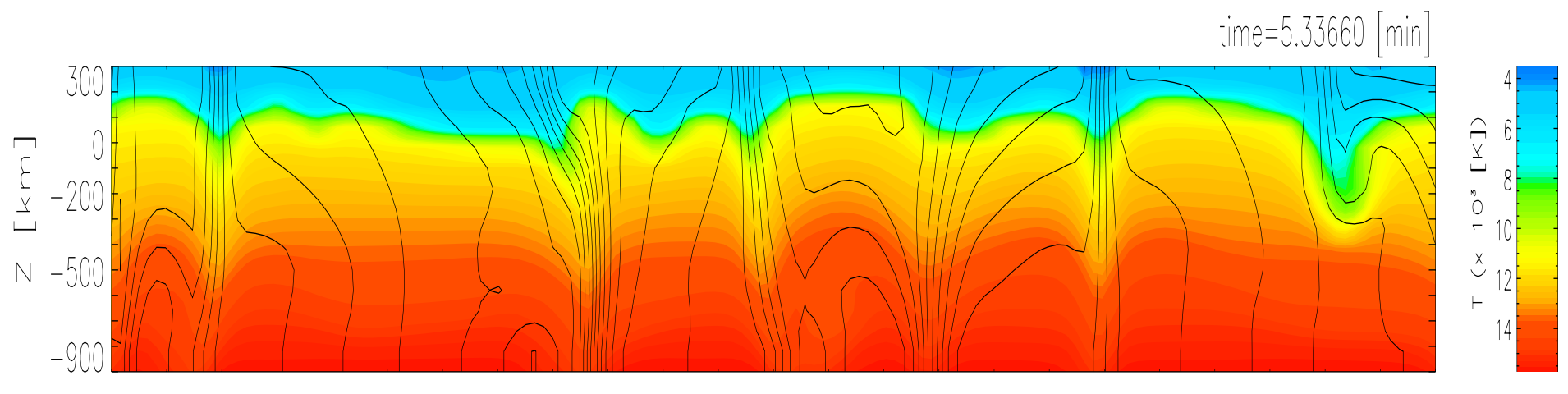

$$
-6000
$$

$-4000$

$-2000$

2000

4000

6000

$x[\mathrm{~km}]$
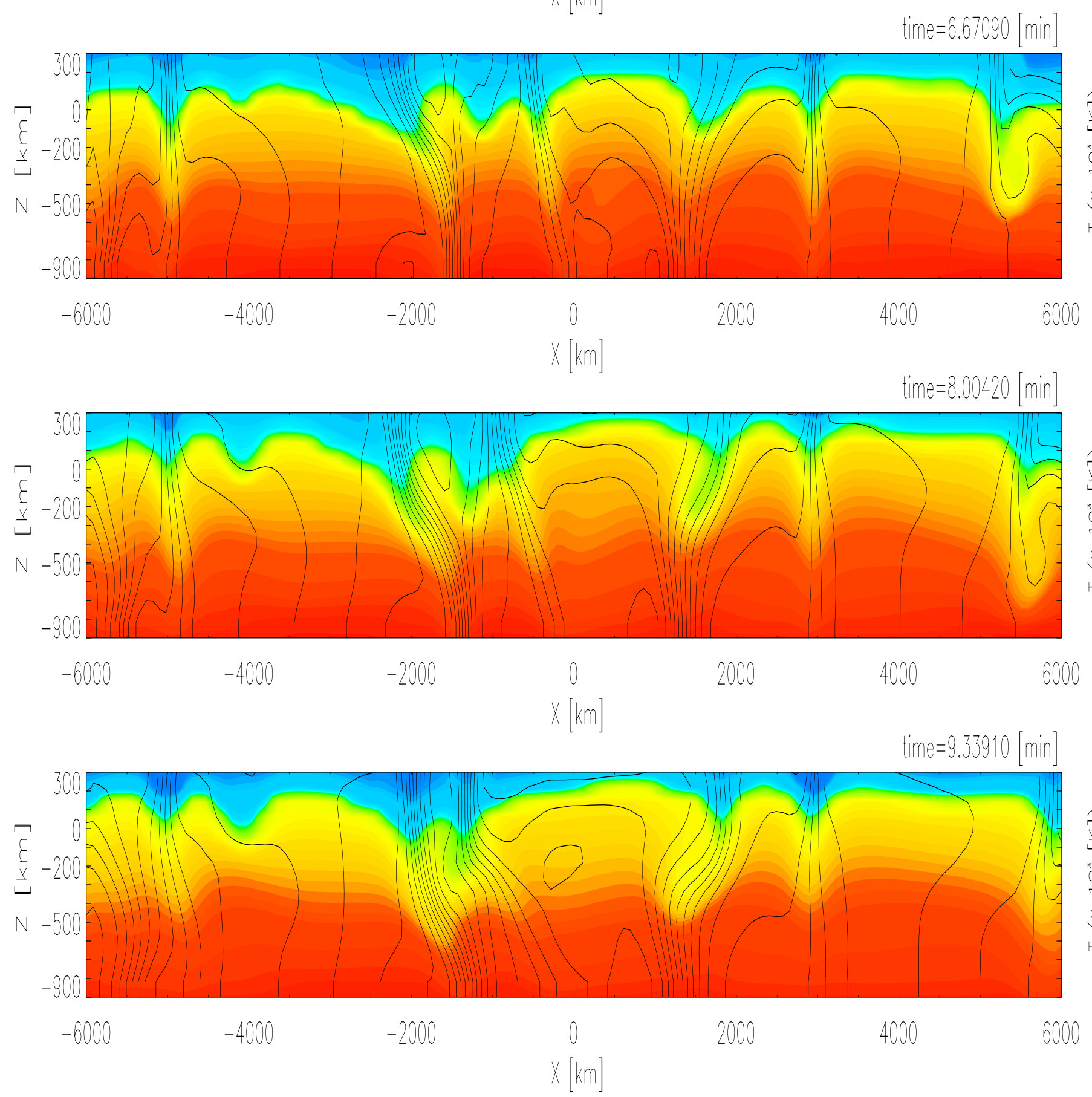

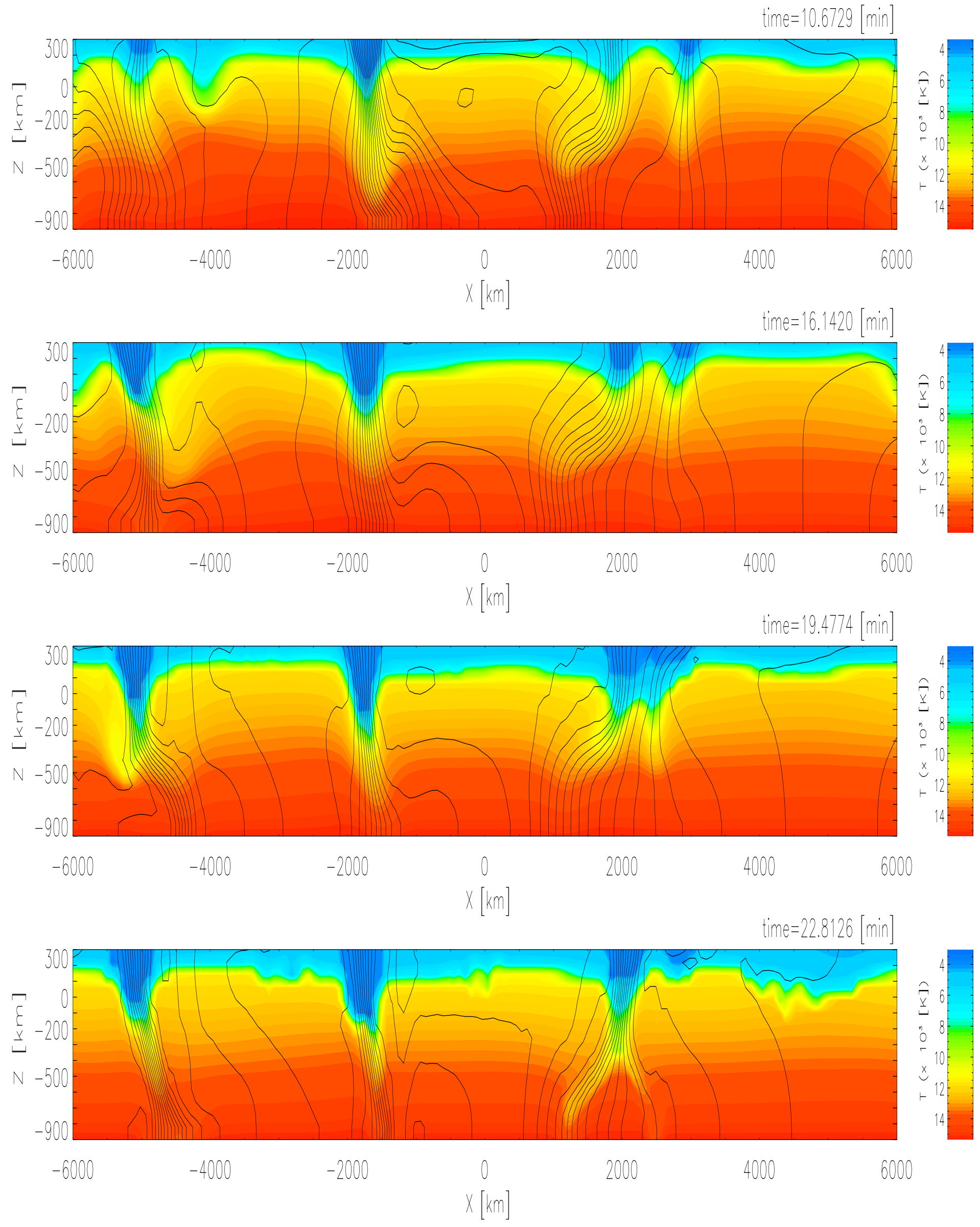

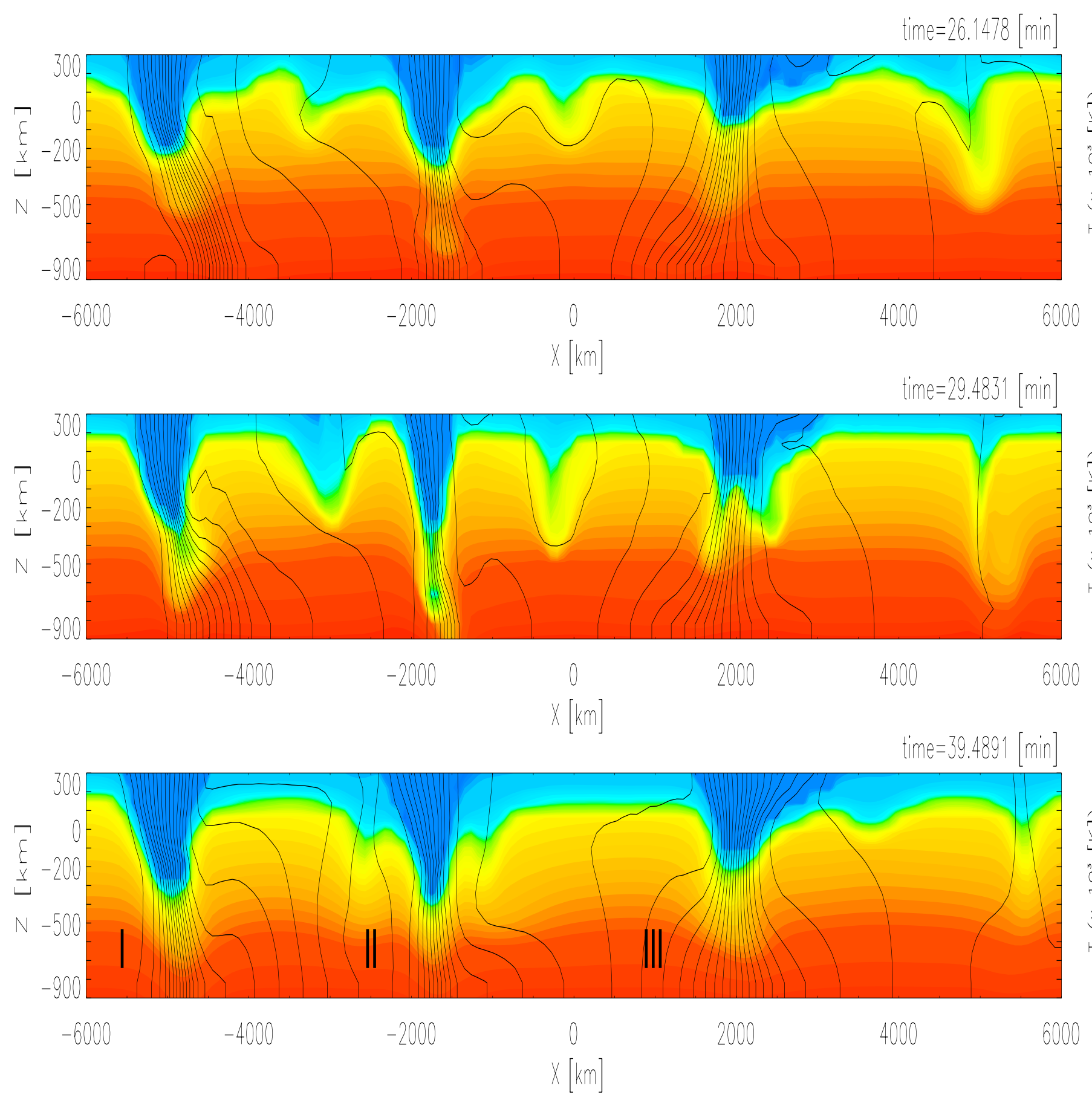

Abbildung 5.1: In den ersten 9 Bildern wird mittels Temperaturisoflächen und Magnetfeldlinien für das gesamte Modellgebiet zu verschiedenen Zeitpunkten die Entstehung von Magnetfeldkonzentrationen durch den sogenannten Flux-expulsion-Prozeß und den sich in der zeitlichen Abfolge daran anschließenden konvektiven Kollaps gezeigt. Die letzten 6 Bilder zeigen die Wechselwirkung zwischen Magnetfeld und konvektiver Strömung zu späteren Zeitpunkten. I, II und III kennzeichnen die später näher untersuchten Magnetfeldkonzentrationen. 
time $=0.00000[\mathrm{~min}]$

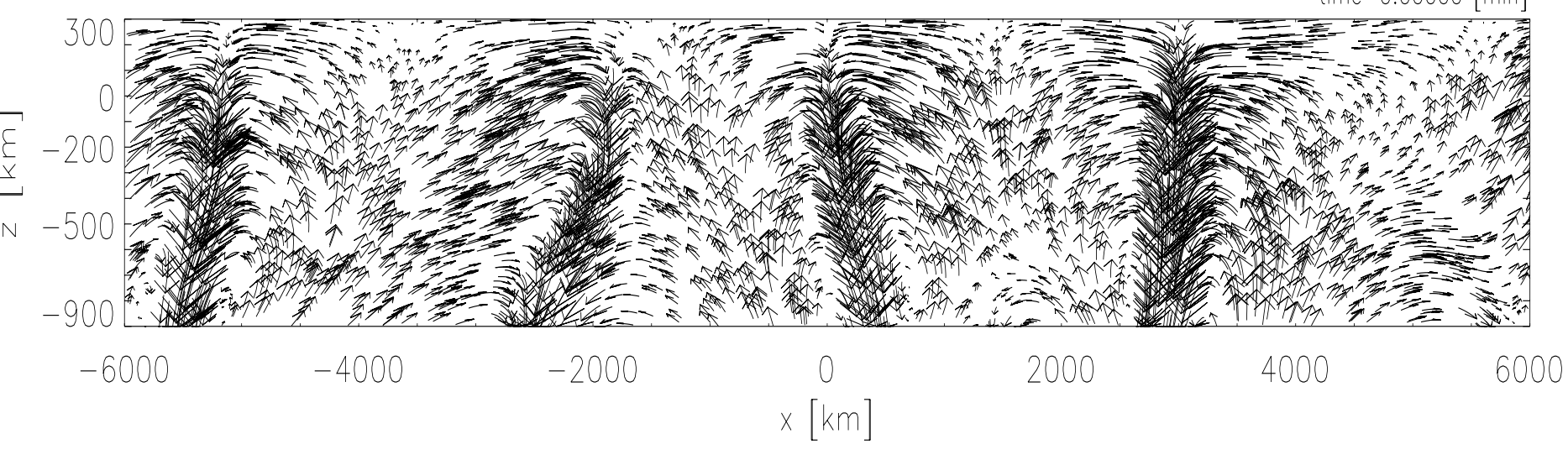

time $=1.33440[\mathrm{~min}]$

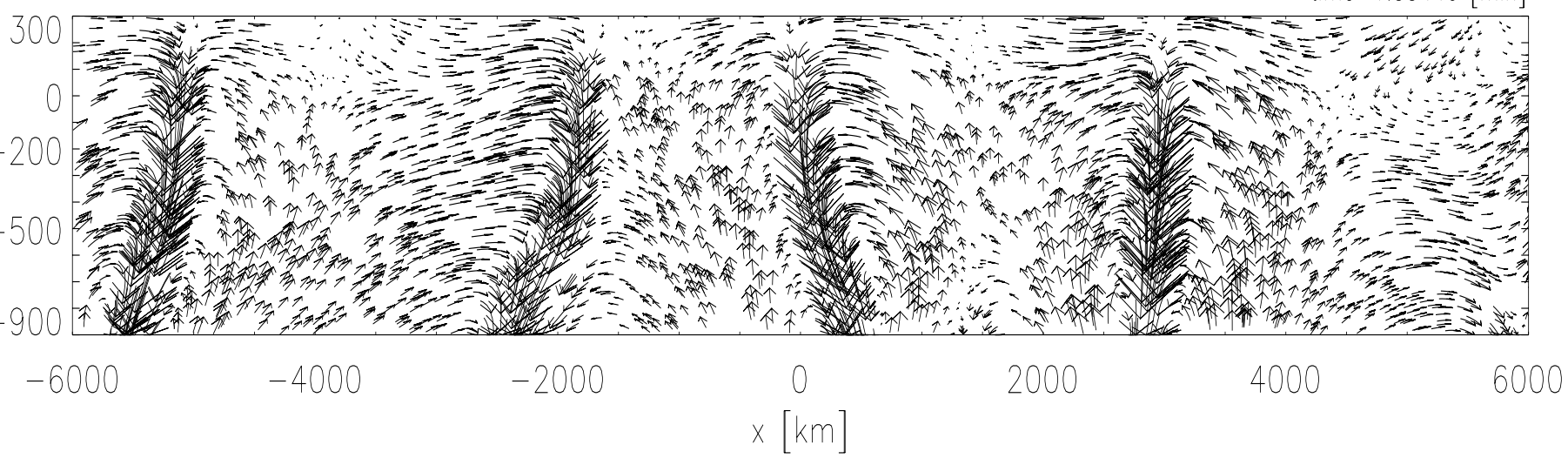

time $=2.66820[\mathrm{~min}]$

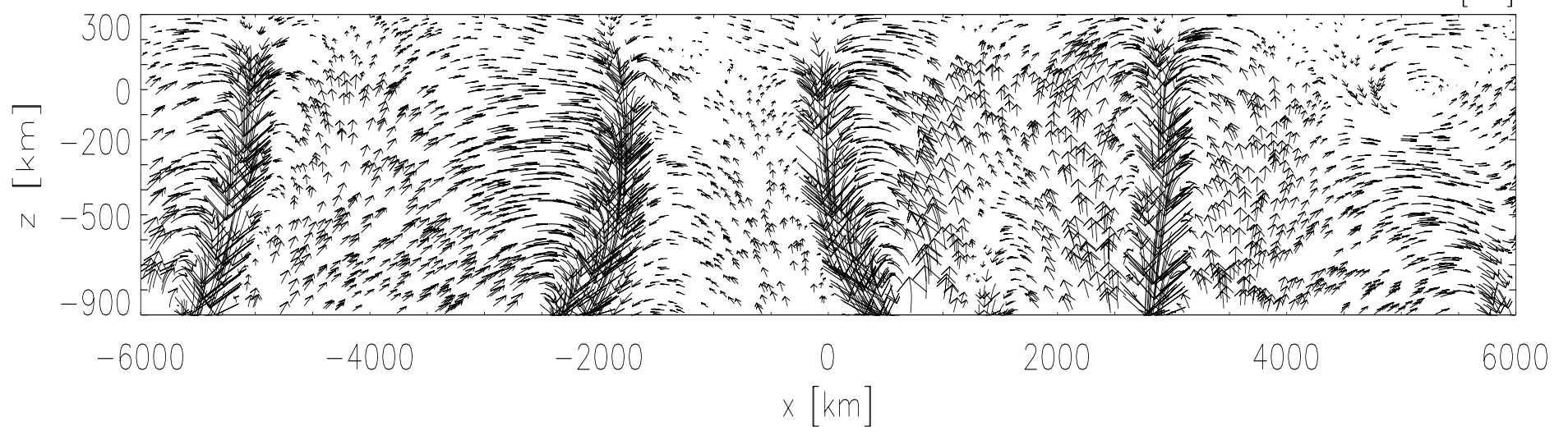

time $=4.00190[\mathrm{~min}]$

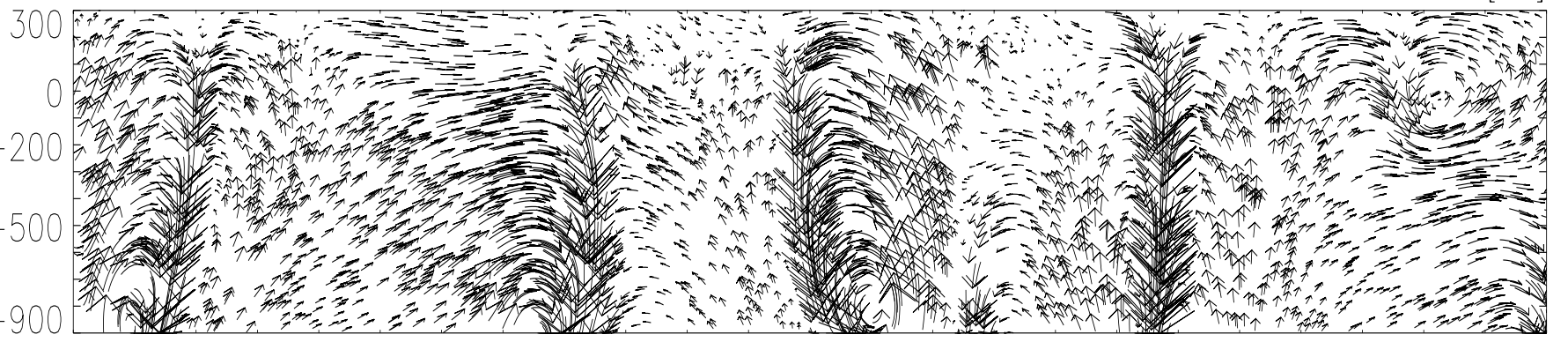

$\begin{array}{ccc}-6000 & -2000 & 0 \\ x[k m] & 2000 & 4000\end{array}$ 


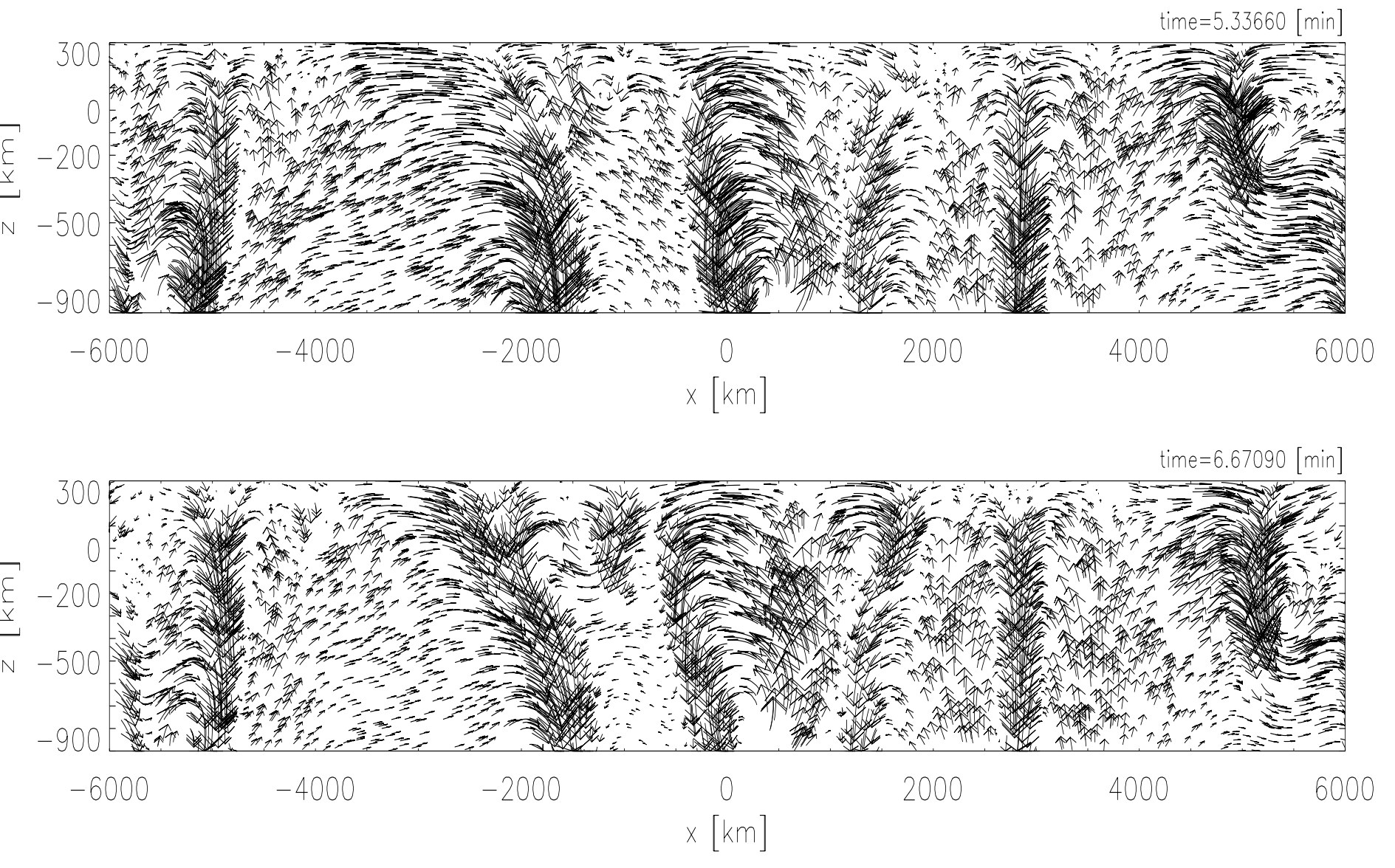

time $=8.00420[\mathrm{~min}]$

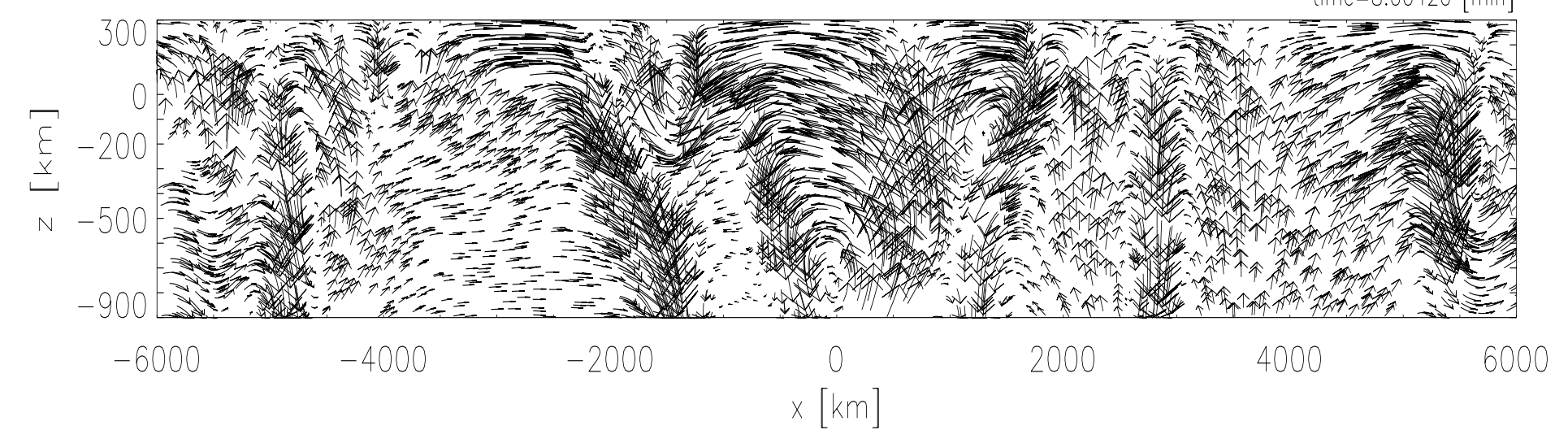

time $=9.33910[\mathrm{~min}]$

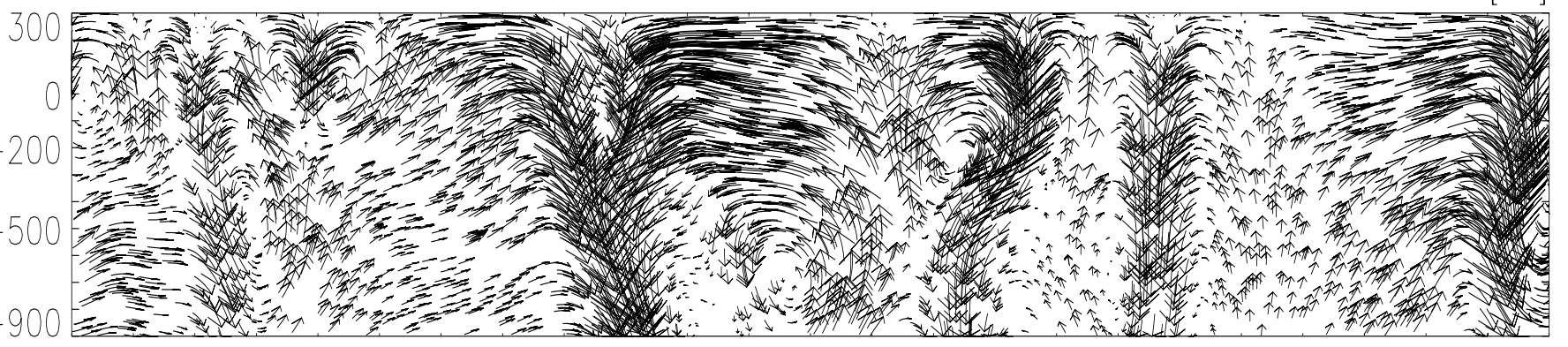

$\begin{array}{cccc}-6000 & -2000 & 0 & 2000\end{array}$

6000 

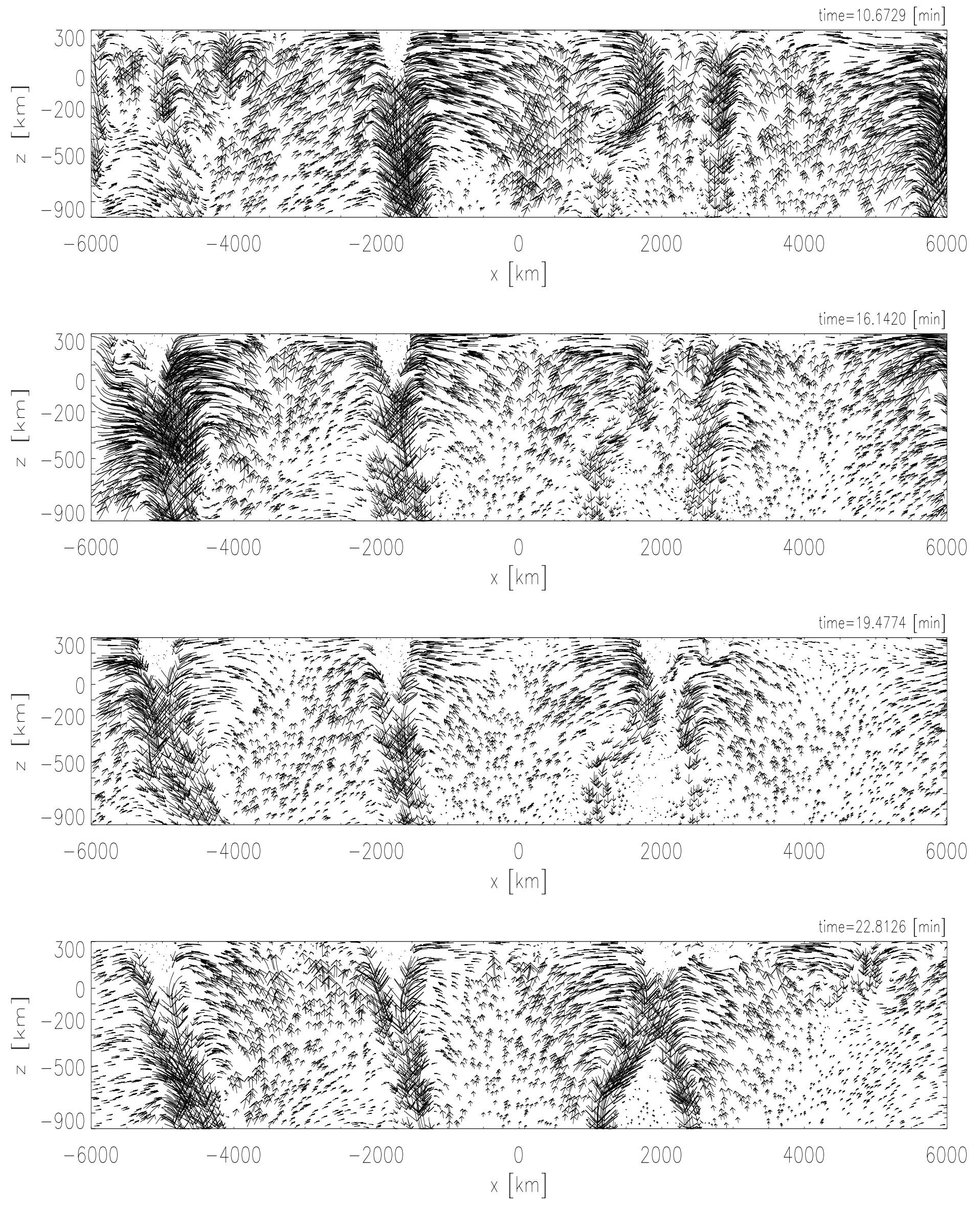

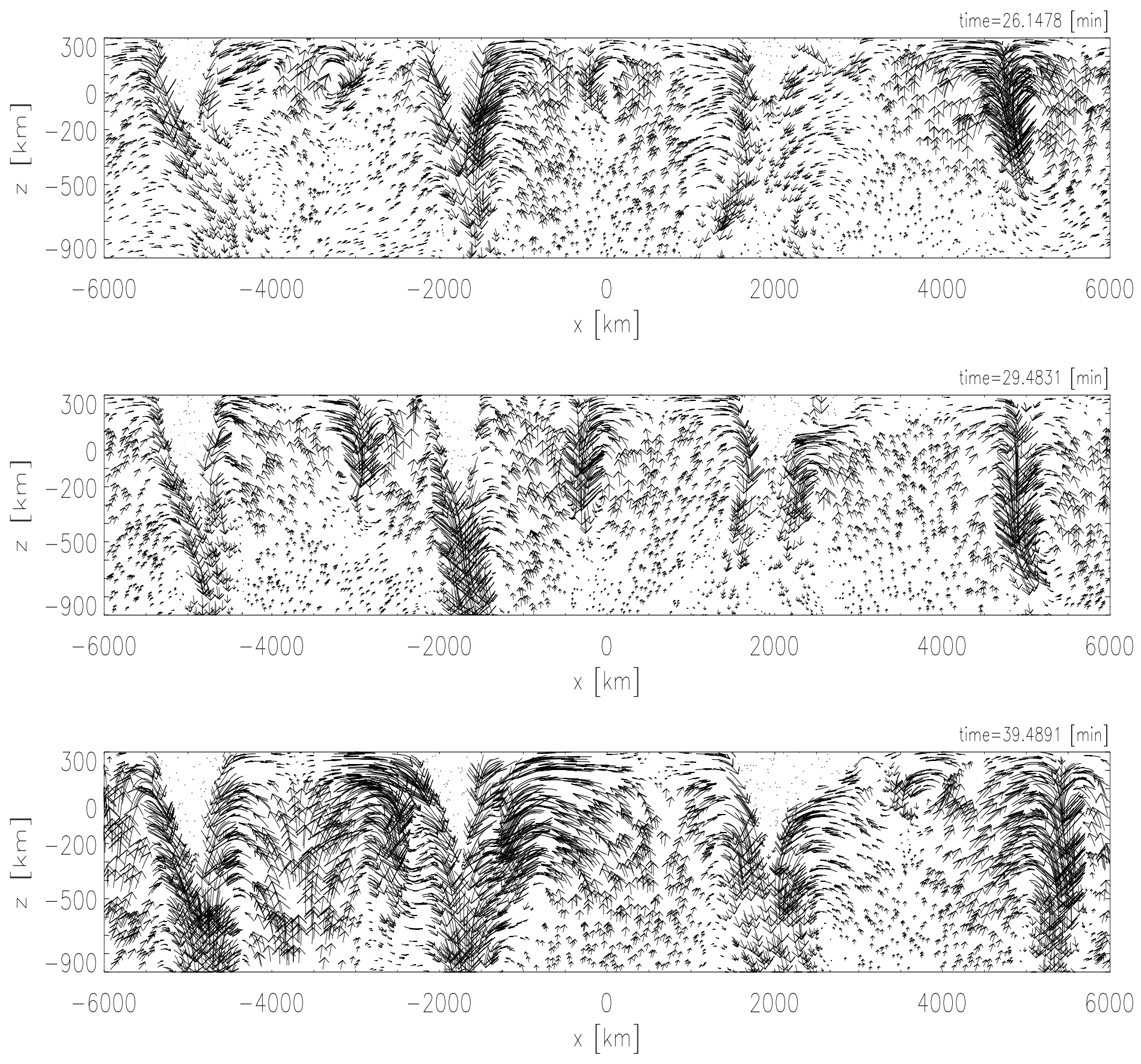

Abbildung 5.2: Gezeigt sind die zu den Abbildungen 5.1 zugehörigen Strömungsmuster. Die maximale Vektorpfeillänge entspricht einer Geschwindigkeit von 10.6 km/s. 
Im Weiteren werden drei magnetische Flußkonzentrationen, die sich innerhalb der ersten 15 Minuten ausgebildet haben, näher untersucht. Sie sind als Magnetfeldkonzentrationen I, II und III bezeichnet, in den horizontalen Bereichen, die von $-6000 \mathrm{~km}$ bis $-3500 \mathrm{~km}$, von $-2500 \mathrm{~km}$ bis $-1000 \mathrm{~km}$ und von $500 \mathrm{~km}$ bis $3000 \mathrm{~km}$ reichen, lokalisiert und haben nach 15 min Simulation bei $z=0$ typischen Breiten von $200 \mathrm{~km}$ (I, II) bzw. $350 \mathrm{~km}$ (III). Die Abbildungen 5.3 und 5.4 stellen für die Magnetfeldkonzentration I die zeitliche Entwicklung der Vertikalkomponente des Magnetfelds $B_{z}$ bei den geometrischen Höhen $z=0$ und $z=200 \mathrm{~km}$ und den Kontinuumsintensitätskontrast $\left(I_{c}-\left\langle I_{c}\right\rangle_{x}\right) /\left\langle I_{c}\right\rangle_{x}$ als Funktion der horizontalen Position dar. $I_{c}$ und $\left\langle I_{c}\right\rangle$ repräsentieren die Kontinuumsintensität bei $5000 \AA$ und ihren horizontalen Mittelwert. Abbildung 5.5 zeigt die Tiefenabhängigkeit des vertikalen Geschwindigkeitsfeld $v_{z}$. Alle Größen sind für 28 verschiedene Zeitpunkte innerhalb der ersten 18 Simulationsminuten dargestellt. Aufgrund der innerhalb der Magnetfeldkonzentrationen unterdrückten Konvektion kommt es in ihren oberen Bereichen zu einer deutlichen Abkühlung und einer dadurch bewirkten starken Abströmung (Abb. 5.5, Zeitpunkte $t_{4}, t_{5}$ ). Dies führt innerhalb der Magnetfeldkonzentration zu einer Evakuierung der oberen Bereiche und einer Feldverstärkung auf 1.5 $\mathrm{kG}$ (Abb. 5.3, Zeitpunkte $t_{7}, t_{11}$ ). Anschließend kommt es, wie in den Abbildungen 5.5 und 5.6 gezeigt im oberen Teil der Magnetfeldkonzentration zu Aufströmungen mit einer maximalen Amplitude von $2 \mathrm{~km} / \mathrm{s}$ (Abb. 5.5, 5.6, Zeitpunkte $t_{8}=5.33$ $\left.\min , t_{12}=8 \mathrm{~min}\right)$. Dies hat die Ursache darin, daß die abströmende Materie in den unteren Flußröhrenbereichen auf Materie größerer Dichte trifft und nach oben laufende Druckwellen bewirkt. Diese führen zu einer Abschwächung der Abströmung innerhalb der mittleren und unteren Flußröhrenbereiche und zu einer Aufströmung in den vorher evakuierten oberen Gebieten. Dadurch heizen die vorher abgekühlten Atmosphärenbereiche auf und die Magnetfeldstärke nimmt wegen des zunehmenden Gasdrucks wieder ab und erreicht nach 8.67 Minuten den Minimalwert von 0.8 $\mathrm{kG}$ (Abb. 5.3, Zeitpunkt $t_{14}$ ). Die weiterhin bestehende radiative Abkühlung bewirkt anschließend wieder eine Abströmung mit anschließender partieller Evakuierung der oberen Bereiche und dem damit verbundenen Anstieg des magnetischen Drucks. Die Feldstärke nimmt nach $t_{11}=7.33$ min den Wert von $1.0 \mathrm{kG}$ an und sinkt anschließend wieder ab. Dies ist wiederum mit Aufströmungen innerhalb der Magnetfeldkonzentration verbunden (Abbn. 5.5 und 5.6). Begleitet wird die $\mathrm{Zu}-$ und Abnahme der Magnetfeldstärke durch Variationen des in Abb. 5.4 dargestellten Kontrasts der Kontinuumsintensität innerhalb der Magnetfeldkonzentration. Da die Atmosphäre in der Magnetfeldkonzentration durch Abstrahlung abgekühlt wird, sinkt das $\tau_{5000}=1$ Niveau in größere geometrische Tiefen ab. Die Umgebung der Magnetfeldkonzentration ist in der gleichen geometrischen Tiefe sehr viel heißer als die Magnetfeldkonzentration und heizt deren Inneres auf. Dadurch wird die Temperatur relativ zur optischen Tiefe in der Umgebung angehoben und die von ihr ausgesandte Kontinuumsintensität innerhalb der Magnetfeldkonzentration steigt relativ zu der der Umgebung an. Zu den Zeiten maximaler Feldstärke $\left(t_{7}, t_{11}\right.$ und $\left.t_{15}\right)$ ist der Kontrast $30 \%$ größer als zu den Zeiten niedrigerer Feldstärke $\left(0.8 \mathrm{kG}, t_{8}\right.$ und $\left.t_{13}\right)$. 


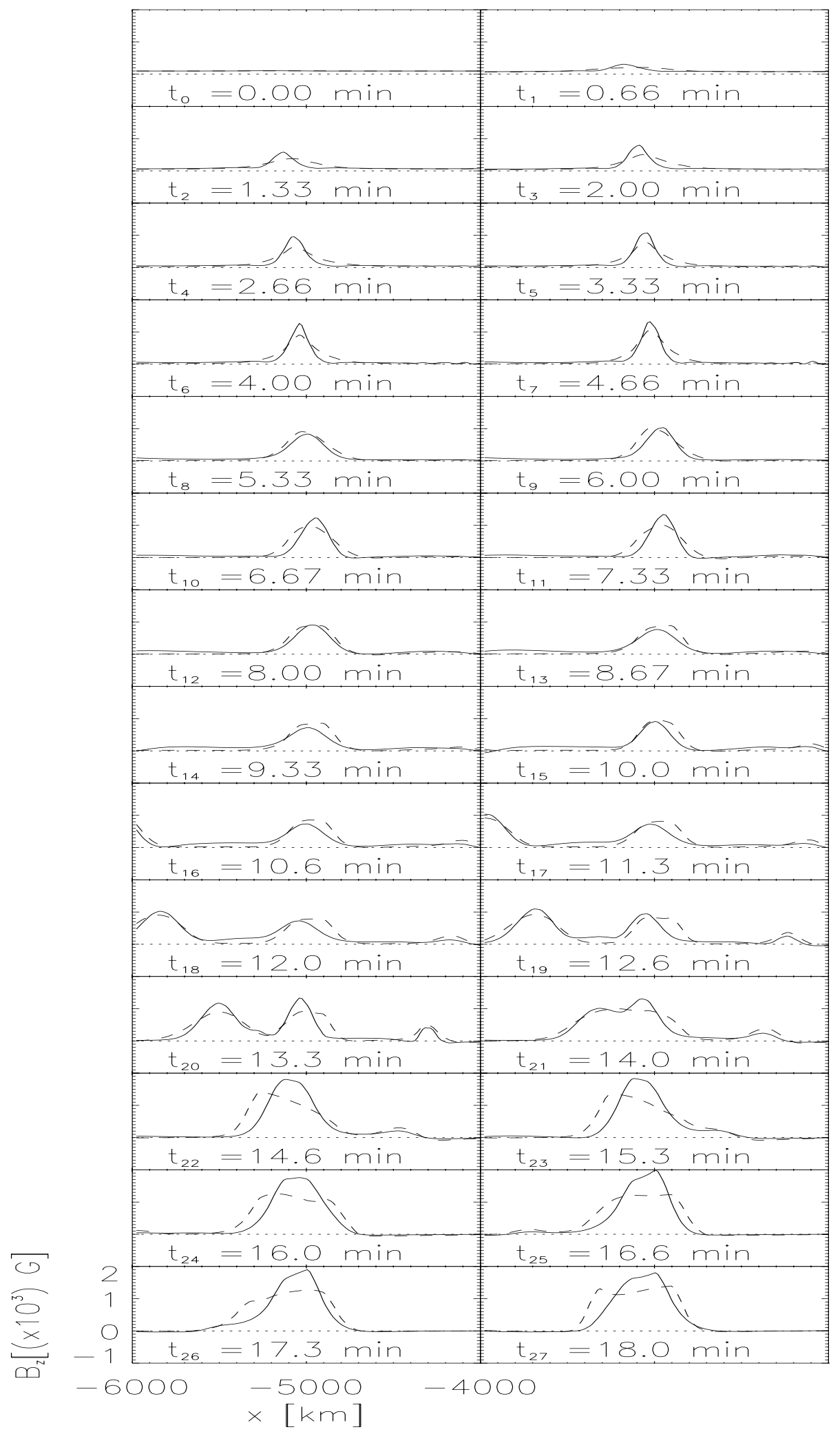

Abbildung 5.3: Die Vertikalkomponente des Magnetfelds für den die Magnetfeldkonzentration I enthaltenden Bereich zu verschiedenen Zeitpunkten für die geometrischen Höhen von $z=0 \mathrm{~km}$ (durchgezogen) und $z=200 \mathrm{~km}$ (gestrichelt). Die gepunktete Linie stellt das Niveau $B_{z}=0$ dar. 


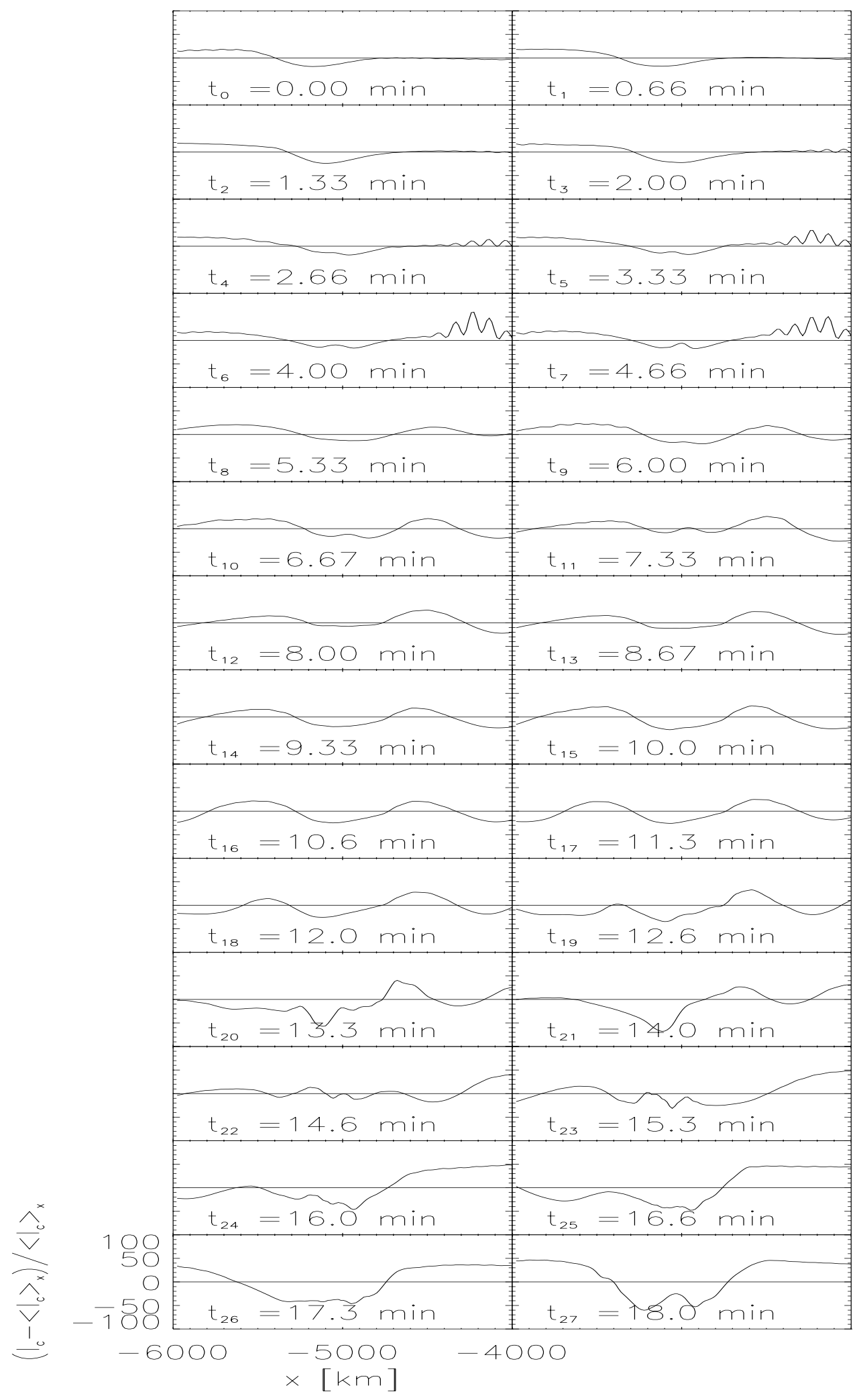

Abbildung 5.4: Kontrast der Kontinuumsintensität für den die Magnetfeldkonzentration I enthaltenden Bereich zu verschiedenen Zeitpunkten. 


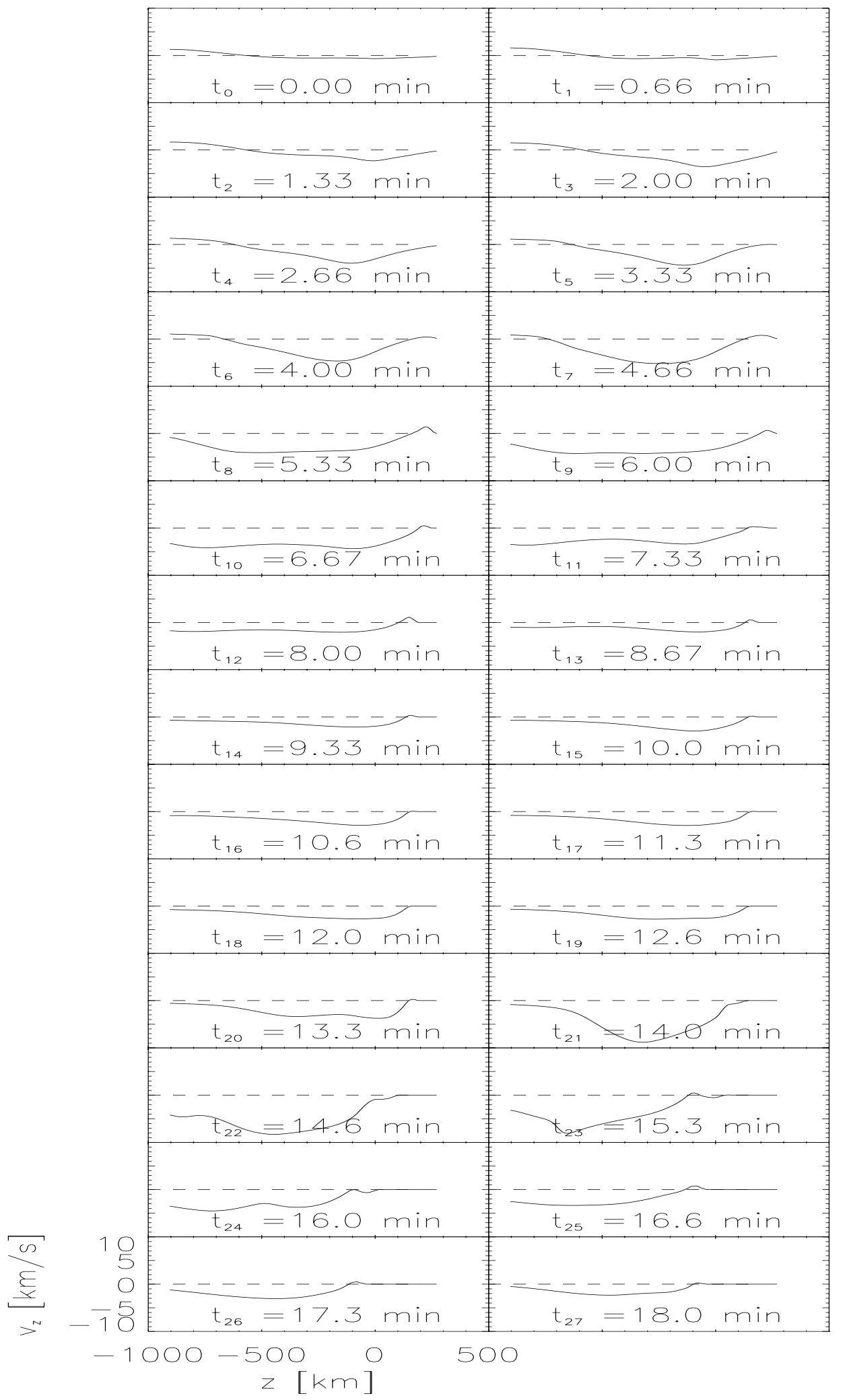

Abbildung 5.5: Tiefenprofil der Vertikalkomponente des Geschwindigkeitsfeld innerhalb der Magnetfeldkonzentration I zu verschiedenen Zeitpunkten. 

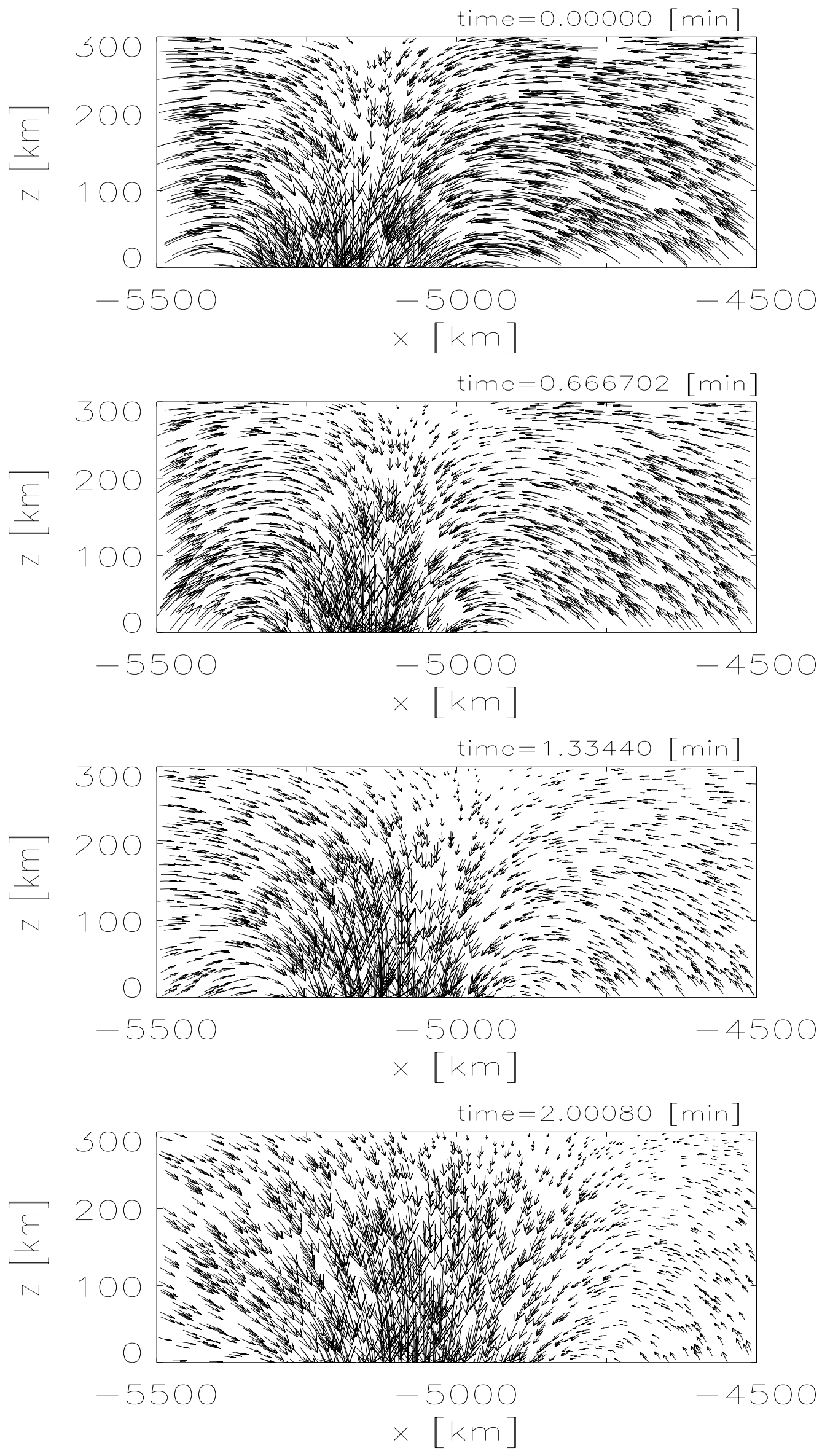

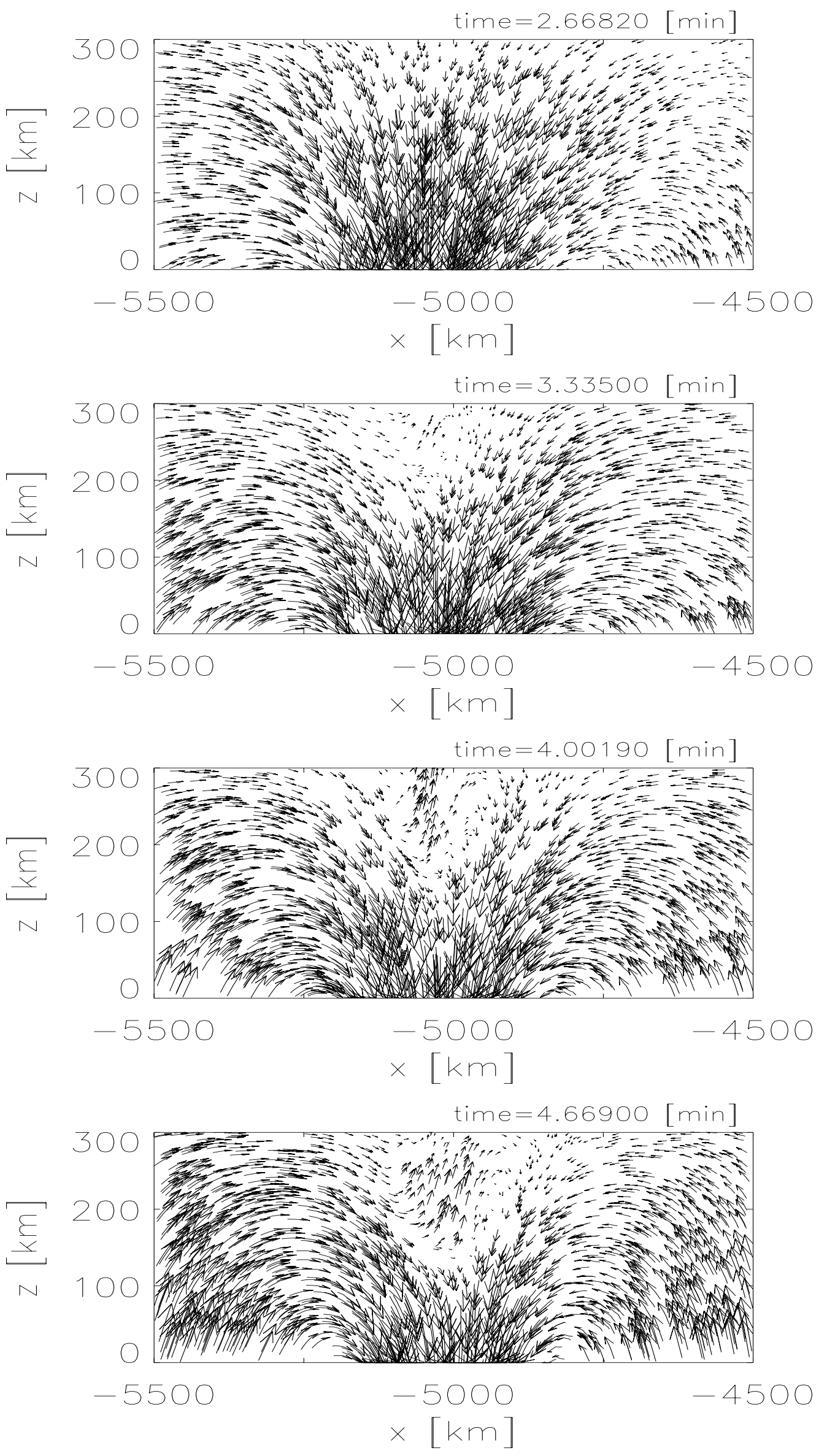

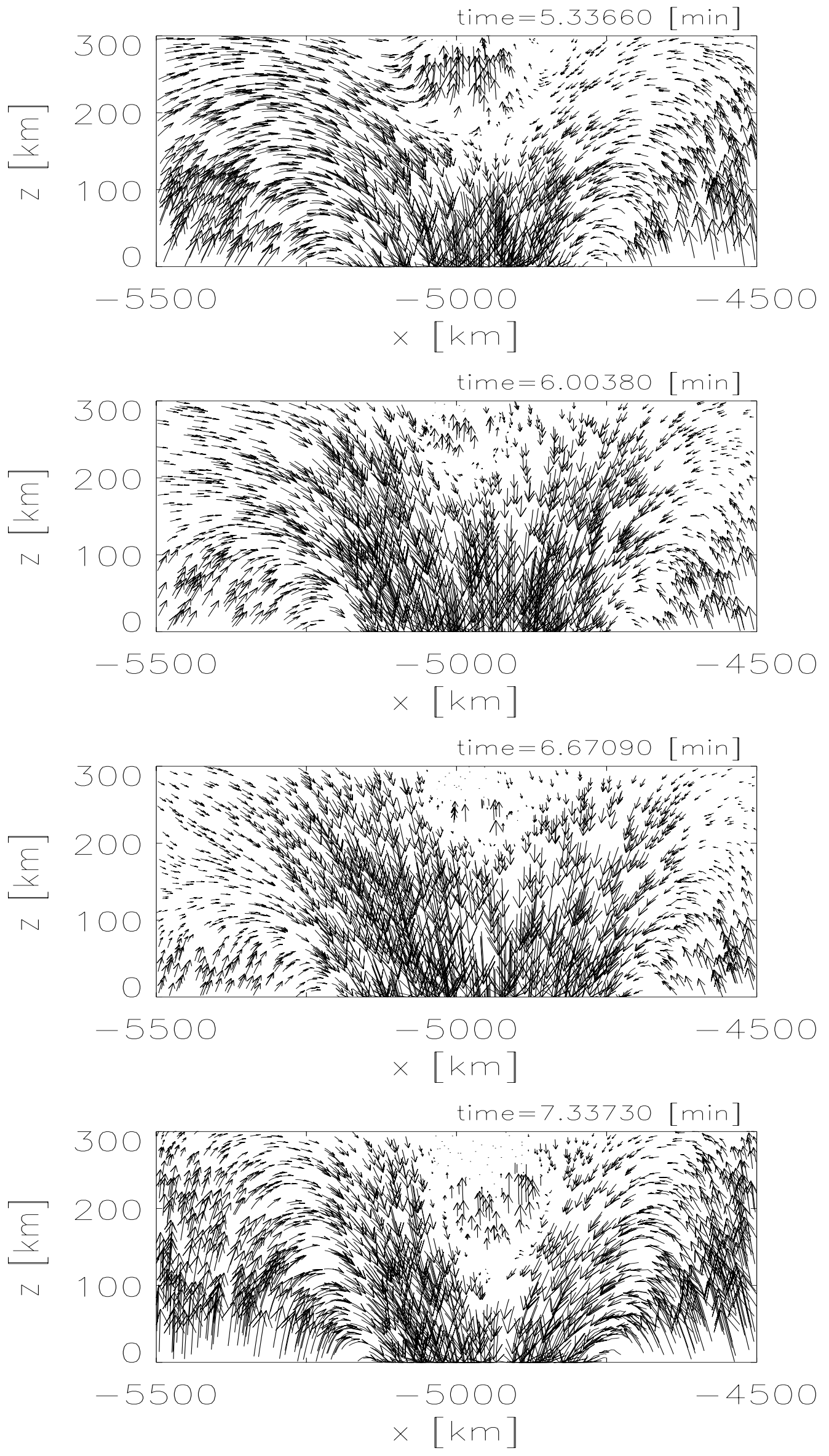

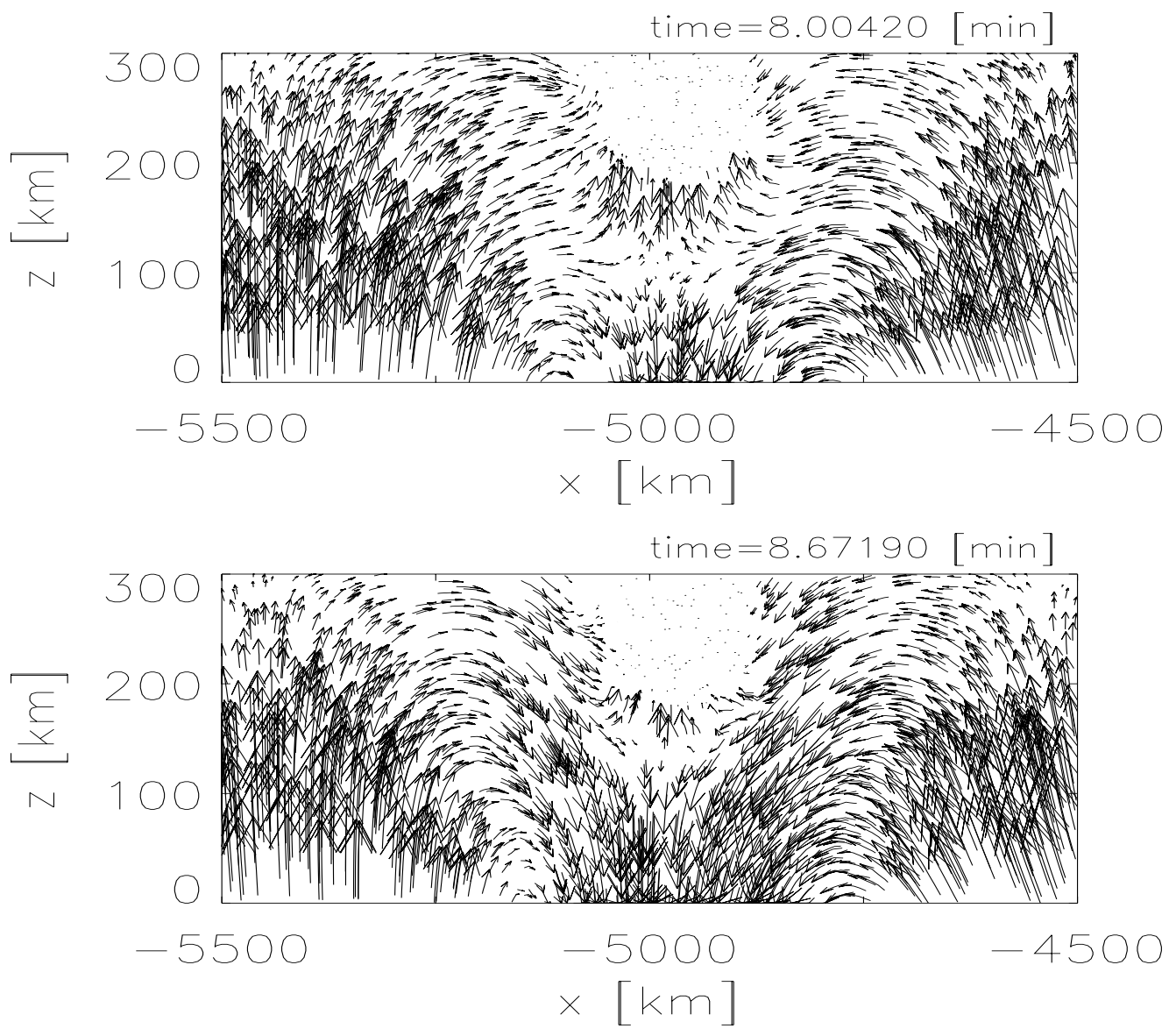

Abbildung 5.6: Gezeigt ist das Strömungsmuster in einem von $-5500 \mathrm{~km}$ bis $-4500 \mathrm{~km}$ horizontal und von $0 \mathrm{~km}$ bis $300 \mathrm{~km}$ vertikal ausgedehnten Bereich, der den oberen Teil der Magnetfeldkonzentration I für die ersten 12 der in Abb. 5.3 gezeigten Zeitpunkte darstellt. Die maximale Vektorpfeillänge entspricht einer Geschwindigkeit von $5.2 \mathrm{~km} / \mathrm{s}$. 
Wie die Abbildungen 5.7, 5.9, 5.8 zeigen, durchläuft die Magnetfeldkonzentration II eine qualitativ vergleichbare Entwicklung. Im Gegensatz zur Magnetfeldkonzentration I ist die Variation des Kontrastes der Kontinuumsintensität allerdings größer und beträgt 70\%. Im Fall der dritten untersuchten Magnetfeldkonzentration (III) ist die Entwicklung der der Magnetfeldkonzentration I, was die typischen Zeitskalen, die Feldstärkemaxima (-minima) und die Variation des Kontrastes der Kontinuumsintensität anbetrifft, vergleichbar.

Nachdem die zweite Magnetfeldverstärkung stattgefunden hatte kam es nach der 14. Minute im Fall I, III und nach der 10. Minute im Fall II zu einer erneuten Magnetfeldverstärkung. Diese ist von starken Abströmungen mit Geschwindigkeitsamlituden von bis zu $8 \mathrm{~km} / \mathrm{s}$ verursacht worden. In der Tiefe $z=0$ betrugen die resultierenden maximalen Feldstärken für die drei untersuchten Flußkonzentrationen (I, II, III) 2.1, 1.9 und 2.3 Kilogauß [kG]. In den letzten 6 Bildern der Abbn. 5.1, 5.2 sind diese Magnetfeldstrukturen und das Geschwindigkeitsfeld für verschiedene Zeitpunkte $t_{n} \geq 10$ min dargestellt. Die Magnetfeldgeometrie und die Magnetfeldstärke ändern sich nach der 14. Minute im Falle der Magnetfeldkonzentrationen I und III und der 10. Minute für II nur unwesentlich. Mit zunehmender Magnetfeldstärke [Abbn. 5.10, 5.11 (linke Spalte)] werden die konvektiven Abströmungen in den oberen Bereichen der Magnetfeldkonzentrationen unterdrückt, werden sehr gering oder verschwinden ganz, wie in den Abbildungen 5.10, 5.11 (mittlere Spalte) für die Magnetfeldkonzentrationen I und II gezeigt, während in der Umgebung der Magnetfeldkonzentrationen starke Abströmungen mit Amplituden von bis zu 6 $\mathrm{km} / \mathrm{s}$ weiterhin existent sind [Abbildungen 5.10, 5.11 (rechte Spalte)].

Mit fortschreitender Zeit $(t>14$ min) bleibt die Korrelation zwischen der Zu- und Abnahme des Kontrasts und der Zu- und Abnahme der Magnetfeldstärke im Laufe eines kollapsartigen Prozeß zwar bestehen, jedoch sinkt der Kontrast im Laufe der Simulation ab und wird negativ. Dies hängt von dem magnetischen Fluß der Magnetfeldkonzentrationen ab. Je mehr magnetischer Fluß sich ansammelt und je größer die Feldstärken werden, desto breiter werden die Magnetfeldkonzentrationen. Die typischen horizontalen Ausdehnungen der drei Magnetfeldkonzentrationen entsprachen am Simulationsende denjenigen von kleinen magnetischen Poren oder magnetic knots ([92], [1]). Da die heißere Umgebung die radiative Abkühlung der breiten Magnetfeldkonzentrationen nicht mehr ausgleichen und deren Inneres aufheizen kann, kommt es durch die andauernde radiative Abkühlung zur weiteren Abkühlung des Inneren der Magnetfeldkonzentrationen und zur Abnahme des Kontrasts der Kontinuumsintensität. Dies steht im Einklang mit den von Deinzer et al. (1984) ([17]) gefundenen Resultaten. 


\section{KAPITEL 5. MAGNETFELDVERSTÄRKUNG DURCH KONVEKTION}

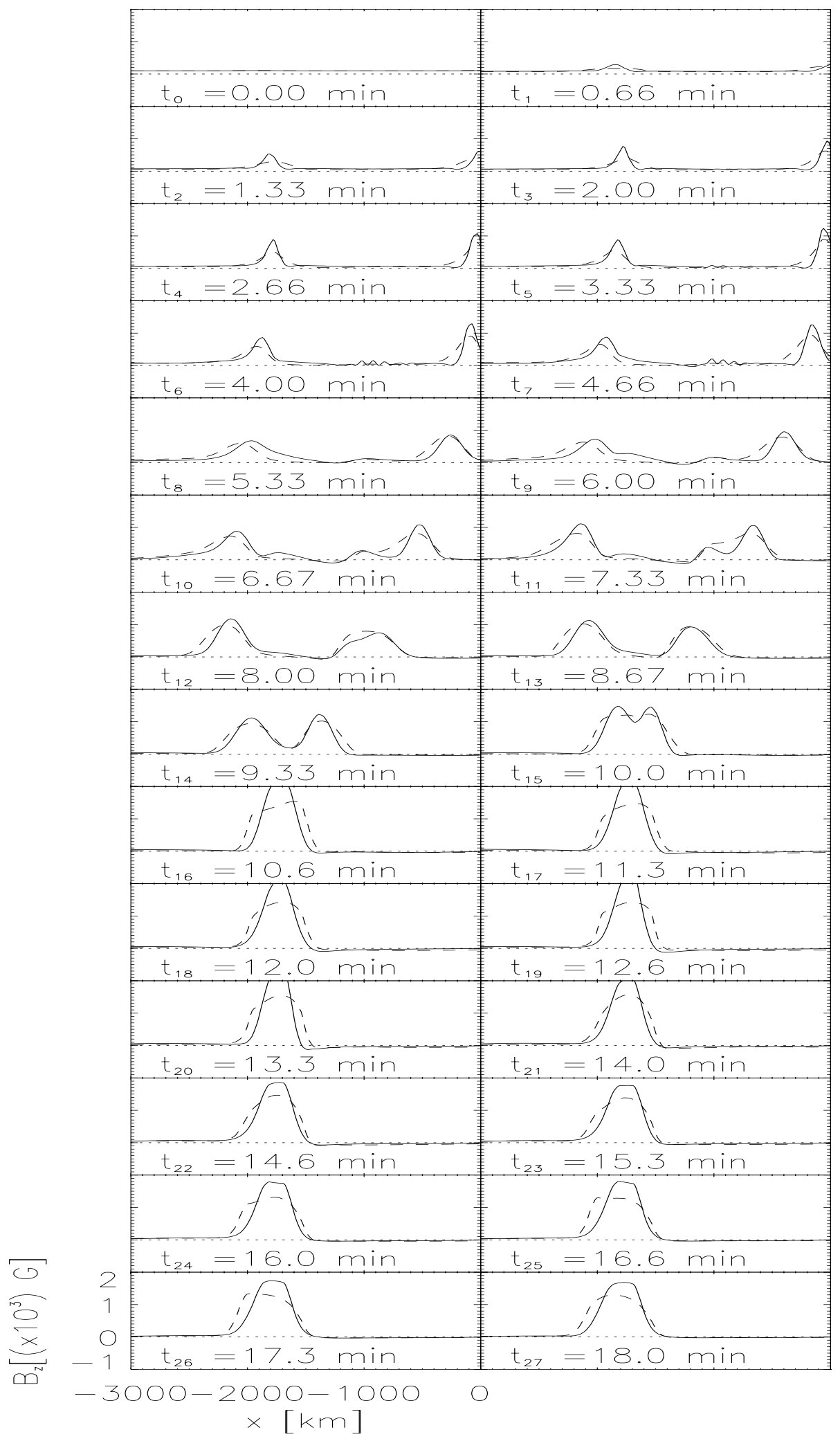

Abbildung 5.7: Die Vertikalkomponente des Magnetfelds für den die Magnetfeldkonzentration II enthaltenden Bereich, zu verschiedenen Zeitpunkten für die geometrischen Höhen von $z=0 \mathrm{~km}$ (durchgezogen) und $\mathrm{z}=200 \mathrm{~km}$ (gestrichelt). 


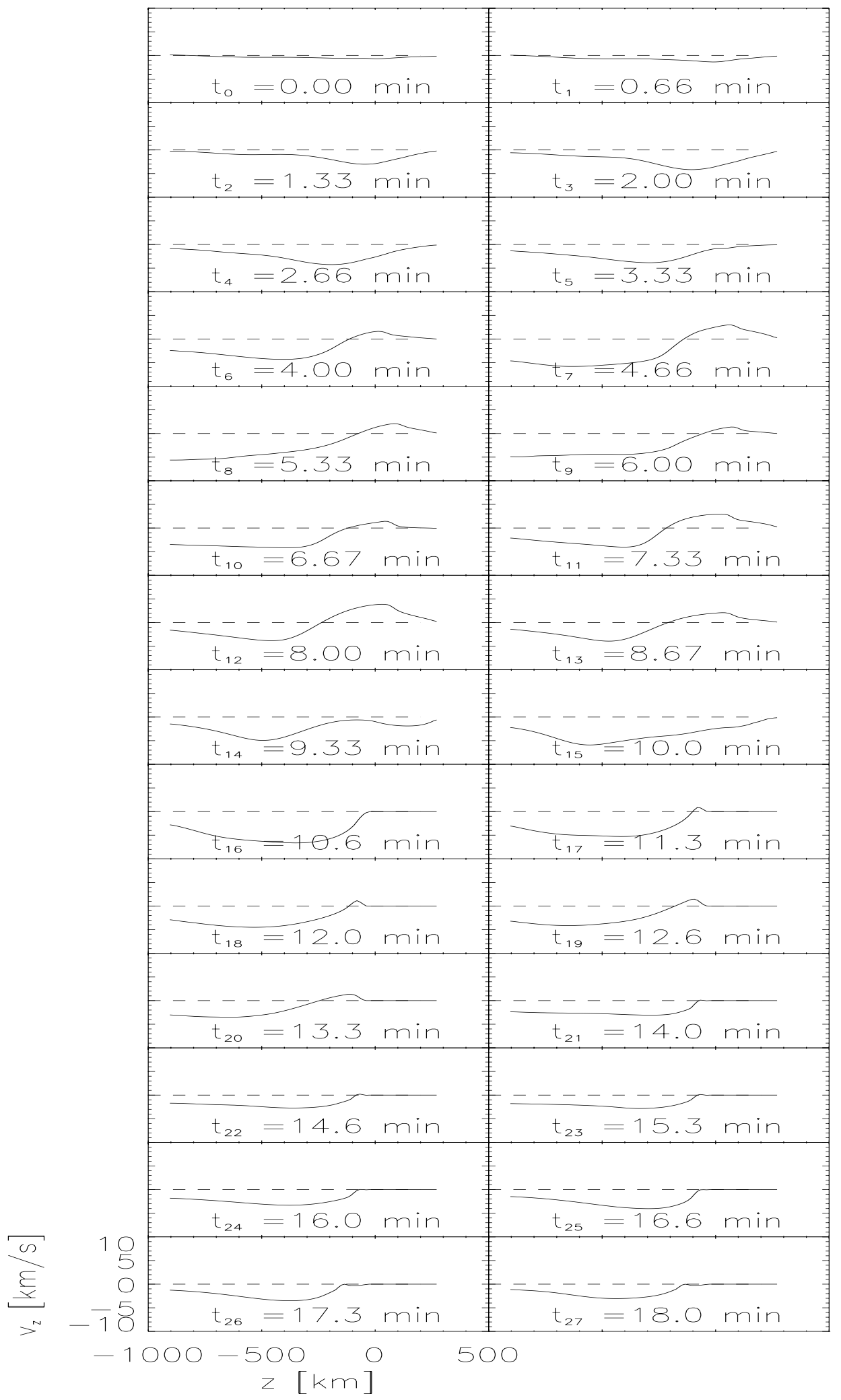

Abbildung 5.8: Tiefenprofil der Vertikalkomponente des Geschwindigkeitsfeld innerhalb der Magnetfeldkonzentration II zu verschiedenen Zeitpunkten. 


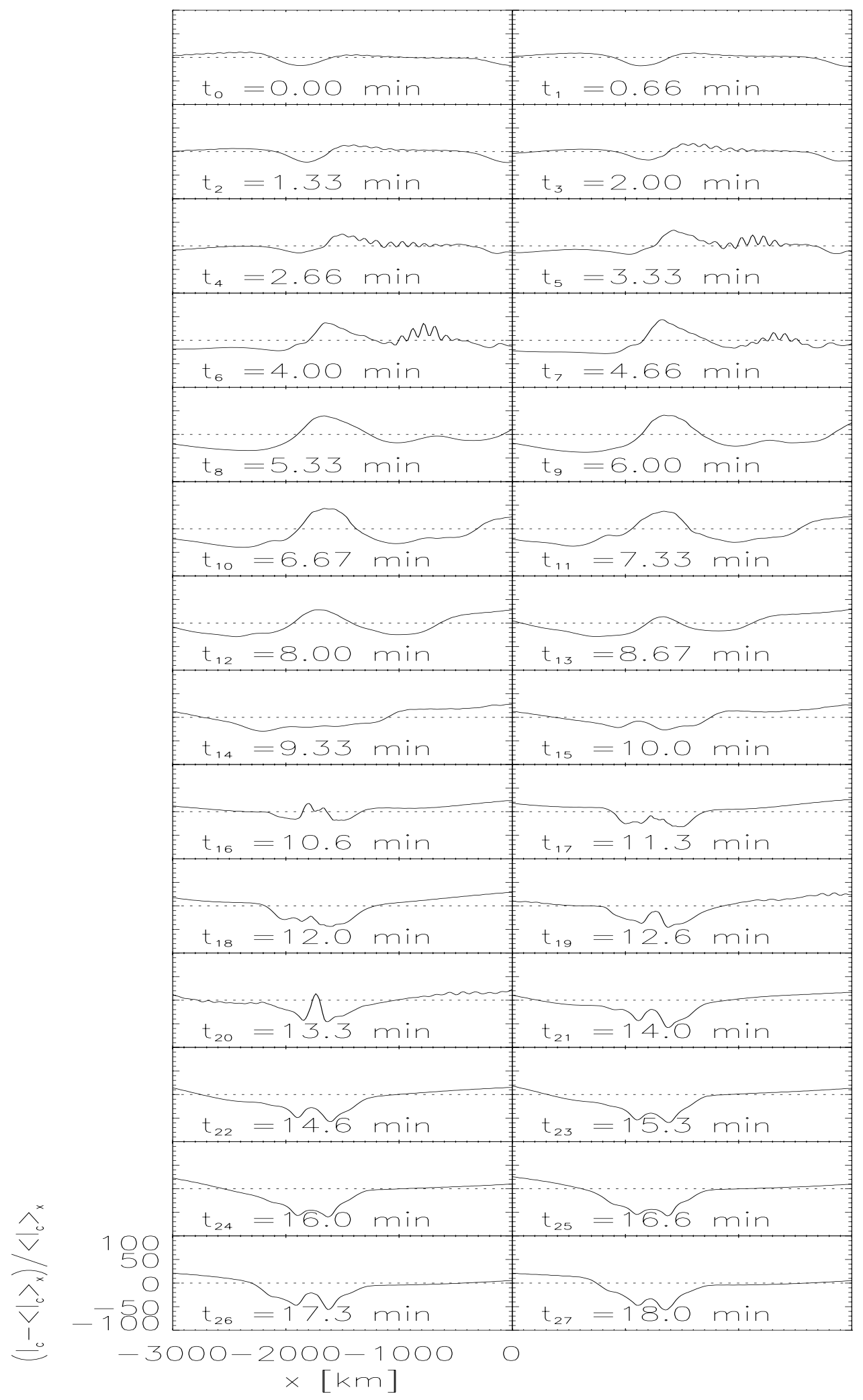

Abbildung 5.9: Kontinuumsintensitätskontrast für den die Magnetfeldkonzentration II enthaltenden Bereich, zu verschiedenen Zeitpunkten. 

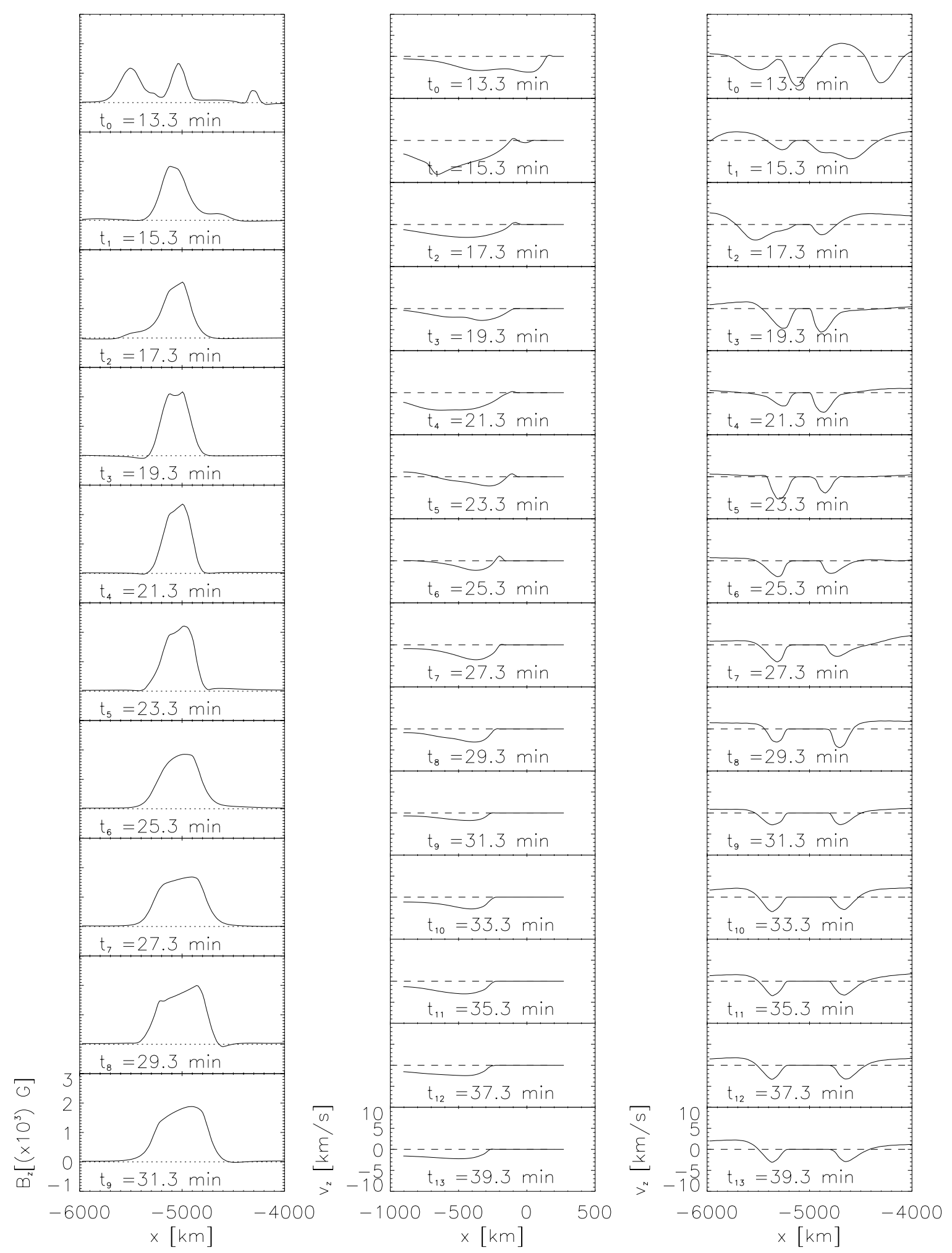

Abbildung 5.10: Links: Die Vertikalkomponente des Magnetfelds für die Magnetfeldkonzentration I zu verschiedenen Zeitpunkten $t_{n}>10 \mathrm{~min}$ bei $z=0 \mathrm{~km}$. Mitte: Tiefenabhängigkeit der Vertikalkomponente der Geschwindigkeit. Rechts: Horizontale Abhängigkeit der Vertikalkomponente der Geschwindigkeit bei $\mathrm{z}=0 \mathrm{~km}$. 

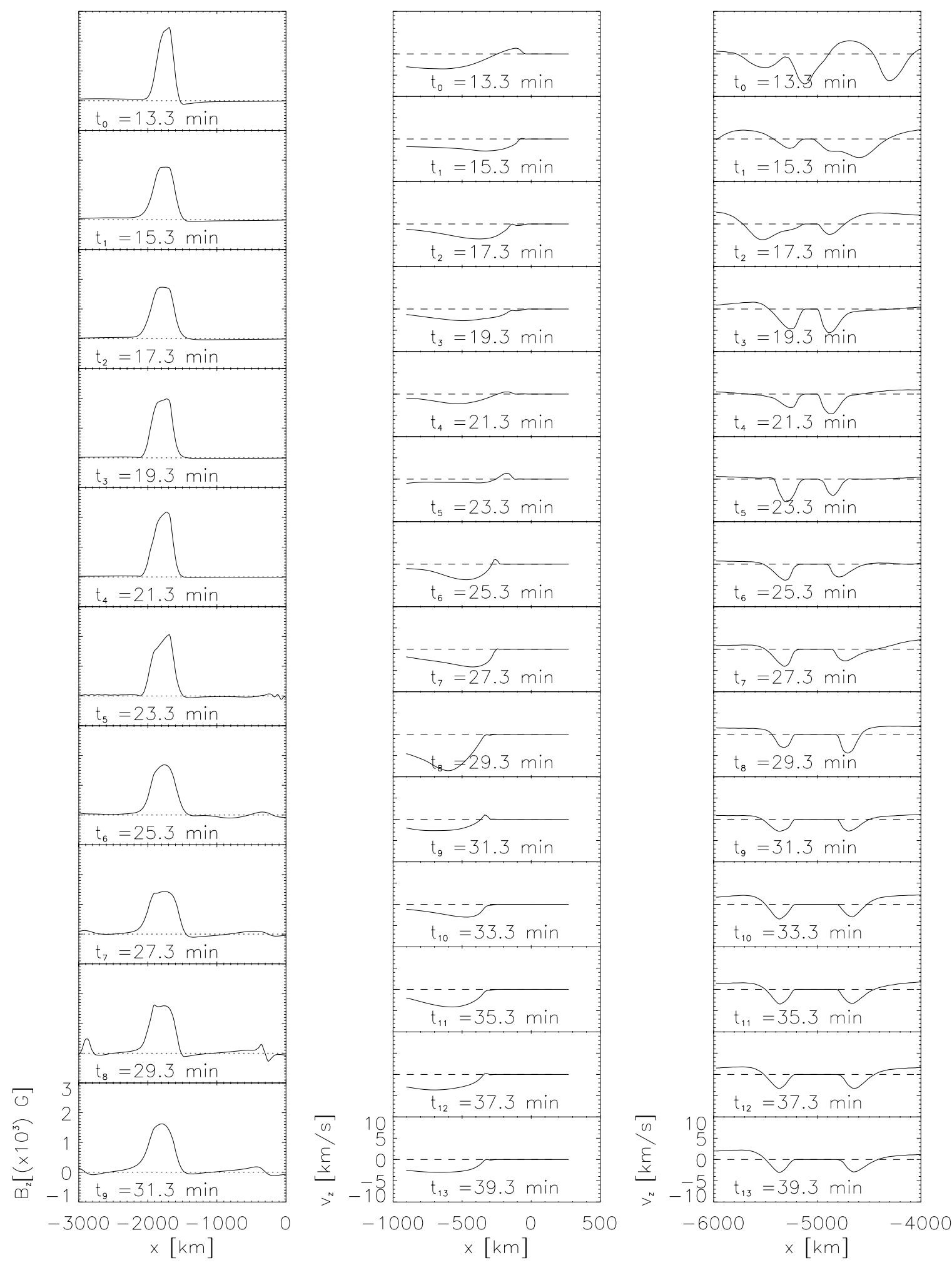

Abbildung 5.11: Links: Die Vertikalkomponente des Magnetfelds für die Magnetfeldkonzentration II zu verschiedenen Zeitpunkten $t_{n}>10$ min für $z=0 \mathrm{~km}$. Mitte: Tiefenabhängigkeit der Vertikalkomponente der Geschwindigkeit. Rechts: Horizontale Abhängigkeit der Vertikalkomponente der Geschwindigkeit bei $\mathrm{z}=0 \mathrm{~km}$. 


\subsubsection{Diskussion}

Im Vergleich zu den von Grossmann-Doerth et al. (1998) ([36]) durchgeführten Simulationen sind die typischen Zeitskalen innerhalb der der konvektive Kollaps abläuft, länger. Bei Grossmann-Doerth et al. beträgt für ein $100 \mathrm{Gauß} \mathrm{starkes} \mathrm{ho-}$ mogenes Anfangsmagnetfeld die Zeitspanne bis der maximale Kontrast der Kontinuumsintensität erreicht wird und die maximale Feldverstärkung stattgefunden hat zwischen 2 und 3 Minuten, während es für die von mir untersuchten Magnetfeldkonzentrationen I, II und III etwa 5 Minuten dauerte bis sich das erste Mal eine Magnetfeldverstärkung mit einer resultierenden Feldstärke von $1.5 \mathrm{kG}$ eingestellt hat. Die weiteren Feldverstärkungen konnten Grossmann-Doerth et al. nicht finden, da die von ihnen simulierte Zeitspanne von 5 min zu kurz war. Hasan (1985) ([38]) fand überstabile Oszillationen mit einer Periode von $10^{3} \mathrm{~s}$.

In den hier simulierten 60 Minuten finden innerhalb der ersten 15 Minuten für alle drei Magnetfeldkonzentrationen vier Feldverstärkungen mit resultierenden Feldstärken im Kilogaußbereich statt. Abbildung 5.12 zeigt die zeitliche Variation der Magnetfeldstärke für die Magnetfeldkonzentration I bei z=0. Die erste Feldverstärkung findet nach etwa $4 \mathrm{~min}$, die zweite nach 7-8 min, die dritte nach 10-11 min und die vierte nach 13-14 min statt. Danach finden zwar noch zwei weitere deutliche Feldverstärkungen statt, die allerdings nicht weiter untersucht wurden, da sie keinen Übergang von der Äquipartitionsfeldstärke von $0.5 \mathrm{kG}$ zur Feldstärke von 1 $\mathrm{kG}$ beschreiben, sondern einen Übergang von 1.5 nach $2.4 \mathrm{kG}$, der den Übergang von einer Flußröhre zur kleinen Poren darstellt.

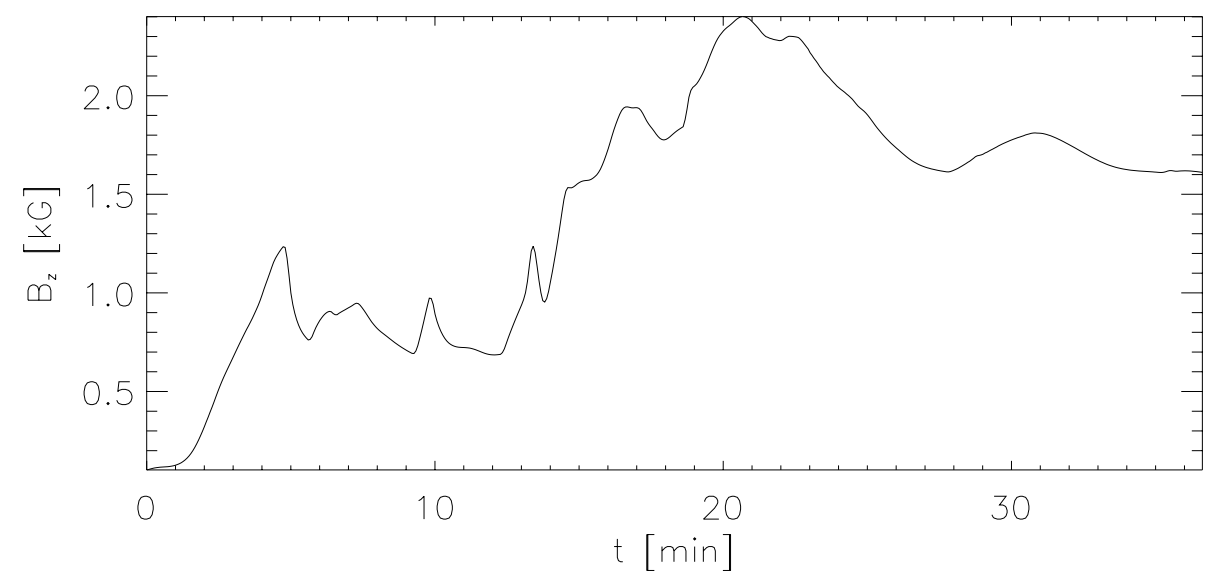

Abbildung 5.12: Zeitliche Abhängigkeit der Magnetfeldstärke für die Magnetfeldkonzentration I bei $\mathrm{z}=0 \mathrm{~km}$. 
Die radiative Abkühlung innerhalb der Magnetfeldkonzentrationen und der konvektive Kollaps bewirken starke Abströmungen, die zur partiellen Evakuierung der Magnetfeldkonzentrationen führen. Die abströmende Materie trifft in den unteren Flußröhrenbereichen auf Materie größerer Dichte, was nach oben laufende Druckwellen bewirkt. Diese verursachen einerseits eine Abschwächung der Abströmung innerhalb der mittleren und unteren Flußröhrenbereiche und andererseits eine Aufströmung in den vorher verdünnten oberen Gebieten. Dadurch heizen sich die vorher abgekühlten Atmosphärenbereiche auf und die Magnetfeldstärke nimmt wegen des zunehmenden Gasdrucks wieder ab. Unter dem Einfluß andauernder radiativer Kühlung wiederholt sich dieser Vorgang und das System wird zu Schwingungen angeregt, welche im Laufe der Simulation allerdings sehr starker Dämpfung unterliegen, deren mögliche Ursache die numerischen Diffusion sein könnte. In letzter Konsequenz führt dies dazu, daß die in den ersten 15 Minuten annährend periodisch stattfindenden Feldverstärkungen unterbunden bzw. stark abgeschwächt werden und bei der im Kap. 6.3.2 beschriebenen Schwingungsanalyse nicht mehr auftauchen. 


\section{Kapitel 6}

\section{Eigenschwingungen}

\subsection{Globale Schwingungsmuster der Sonne}

In der solaren Photosphäre und Chromosphäre existieren globale Schwingungsmuster, die einen einen großen räumlichen Wellenzahlbereich überdecken und von Leighton et al. 1962 entdeckt ([51]) worden sind. Ihre Perioden liegen zwischen 3 min und 12 min. Sie werden auch als 5-Minuten-Oszillationen bezeichnet, da sie bei entsprechenden $3.3 \mathrm{mHz}$ die größte Amplitude besitzen. Die korrespondierenden Geschwindigkeitsamplituden liegen zwischen $0.3-1.6 \mathrm{~km} / \mathrm{s}$. In der Photosphäre sind ihre typischen horizontalen Wellenlängen zwischen 20-40 Mm groß und somit erheblich größer als die horizontalen Ausdehnungen der dort vorherrschenden granularen Strukturen.

Die Sonne ist in guter Näherung eine Gaskugel die sich im hydrostatischen Gleichgewicht befindet. Kleine Störungen der stabilen Gleichgewichtskonfiguration rufen Rückstellkräfte hervor, die die Sonne um die Gleichgewichtslagen schwingen lassen. Unter Vernachlässigung des Magnetfeldes sind diese Rückstellkräfte der Druckgradient, welcher aus einer Kompression oder der Verdünnung eines Gasvolumens herrührt, und der durch die Gravitation bedingte Auftrieb eines ausgelenkten Gasvolumens. Im Falle kleiner Störungen sind die zugehörenden Wellen die Schallwellen oder $p$-Moden und die Schwerewellen oder $g$-Moden. Im allgemeinen ist weder der Zustand reiner Schallwellen, noch der reiner Schwerewellen realisiert, sondern Schwingungen, die entweder von der einen oder der anderen Rückstellkraft dominiert werden. Die nachfolgenden Ausführungen stellen einige Aspekte der Theorie solarer Schwingungen dar [ s.a. Cox (1980) ([8]) und Stix (1991) ([92])].

Ausgangspunkt einer formalen Beschreibung solarer Eigenschwingungen sind die hydrodynamischen Grundgleichungen, welche aus den magnetohydrodynamischen Grundgleichungen Gln. (2.5) unter der Annahme, daß kein Magnetfeld vorhanden ist, folgen. Nimmt man an, daß es sich um adiabatische Störungen handelt, gilt in erster Näherung folgender Zusammenhang zwischen einer Druck- und einer Dich- 
teschwankung:

$$
\frac{\delta P(z)}{P_{0}(z)}=\Gamma_{1} \frac{\delta \rho(z)}{\rho_{0}(z)} .
$$

$P_{0}$ und $\rho_{0}$ stellen hierbei den ungestörten Druck und die ungestörte Dichte dar. $\Gamma_{1}$ ist der Adiabatenexponent:

$$
\Gamma_{1}(z)=\left(\frac{\mathrm{d} \ln P(z)}{\operatorname{dln} \rho(z)}\right)_{S},
$$

der zur adiabatischen Schallgeschwindigkeit in folgendem Verhältnis steht:

$$
c^{2}(z)=\Gamma_{1}(z) \frac{P_{0}(z)}{\rho_{0}(z)} .
$$

Unter der Vernachlässigung von Rotation, großräumigen Geschwindigkeitsfelden und der Viskosität und unter der weiteren Annahme, daß $v / c \ll 1$ ist und alle Terme $\propto(v / c)^{n}(v$ : hydrodynamische Geschwindigkeit, $c$ : adiabatische Schallgeschwindigkeit, $n \geq 2$ ) vernachlässigbar sind, reduziert sich das hydrodynamische Gleichungssystem erheblich und ist linearisierbar. Aus dieser Linearisierung, welche in Stix (1991) ([92]) ausführlich dargestellt ist, ergibt sich ein lineares homogenes Differentialgleichungssystem vierter Ordnung, dessen Lösungsfunktionen als Produkt von zeit-, winkel- und radiusabhängigen Anteilen folgendermaßen dargestellt werden kann:

$$
\vec{\xi}=e^{i \omega t}\left(\xi_{r}(r), \xi_{h}(r) \frac{\partial}{\partial \theta}, \frac{\xi_{h}(r)}{\sin \theta} \frac{\partial}{\partial \phi}\right) Y_{l}^{m}(\theta, \phi) .
$$

Unter Zuhilfenahme der Kugelflächenfunktionen

$$
Y_{l}^{m}(\theta, \phi)=(-1)^{m} c_{l m} P_{l}^{m}(\cos \theta) \exp (i m \Phi)
$$

lassen sich die Schwingungsmuster eines einzelnen Punktes durch den Vektor $\delta \vec{r}(r, \theta, \phi, t)$ beschreiben:

$$
\begin{aligned}
\delta \vec{r}(r, \theta, \phi, t) & =\sum_{l, m}\left[\xi_{r}(r) Y_{l m}(\theta, \phi) \vec{e}_{r}+\xi_{h}(r)\left(\frac{Y_{l m}(\theta, \phi) \vec{e}_{\theta}}{\partial \theta}\right.\right. \\
& \left.\left.+\frac{1}{\sin \theta} \frac{Y_{l m}(\theta, \phi) \vec{e}_{\phi}}{\partial \phi}\right)\right] e^{i \omega t}
\end{aligned}
$$

$\xi_{h}$ und $\xi_{r}$ stellen dabei den horizontalen und vertikalen Anteil der vektoriellen Verschiebung eines Gasvolumens aus seiner Gleichgewichtsposition dar. Die Anzahl der Nullstellen der radialen Eigenfunktion $\xi_{r}$ bezeichnet man dabei als radiale Ordnung $n$.

$P_{l}^{m}$ sind die Legendre Funktionen und $c_{l m}$ der Normalisierungsfaktor:

$$
c_{l m}=\frac{(2 l+1)(l-m)}{4 \pi(l+m) !}
$$


welcher so gewählt wurde, daß das Integral von $\left|Y_{l m}\right|^{2}$ über die Einheitskugel 1 ergibt. $\theta$ repräsentiert die Breiteninformation bzw. beschreibt die Winkelentfernung von der Polachse. $\phi$ hingegen enthält die Längenkreisinformation. Der Grad $l$ (mit $l \geq 0$ und $L=\sqrt{l(l+1)})$ beschreibt die horizontale Wellenzahl $k_{h}$ auf der Oberfläche:

$$
k_{h}=\frac{L}{R}
$$

( $R$ : Sonnenradius)

und ist ein Maß dafür, wieviele Knotenlinien (Längen- und Breitenkreise mit identisch verschwindender Amplitude) die Oberfläche durchziehen. Der Betrag der azimutalen Ordnung $m(-l \leq m \leq+l)|m|$ gibt an, wieviele davon Meridiane sind. Jedes Tripel $(n, l, m)$ beschreibt eine potentielle Schwingungsmöglichkeit (Modus) mit der zugehörigen Frequenz $\nu$, bzw. Kreisfrequenz $\omega$. Unter Verwendung des Lösungsvektor Gl. (6.4) ergibt sich ein System bestehend aus drei Differentialgleichungen, welches sich unter der Annahme, daß Fluktuationen im Gravitationspotential aufgrund von Dichtefluktuationen vernachlässigbar sind, auf zwei Differentialgleichungen erster Ordnung reduziert:

$$
\begin{aligned}
& \frac{1}{r^{2}} \frac{d}{d r}\left(r^{2} \xi_{r}\right)-\frac{\xi_{r} g}{c^{2}}+\frac{1}{\rho_{0}}\left(\frac{1}{c^{2}}-\frac{l(l+1)}{r^{2} \omega^{2}}\right) P_{1}=0 \\
& \frac{1}{\rho_{0}}\left(\frac{d}{d r}+\frac{g}{c^{2}}\right) P_{1}-\left(\omega^{2}-N^{2}\right) \xi_{r}=0
\end{aligned}
$$

Das Lösungsverhalten dieser beiden Gleichungen wird durch eine lokale Analyse mittels asymptotischer Methoden transparent. Eine lokale Analyse bedeutet, daß außer dem Druck und der Dichte $P_{0}$ und $\rho_{0}$, alle Koeffizienten des obigen Gleichungssystems konstant sein sollen. Dies approximiert die Verhältnisse in einem Bereich, in dem sich das Gleichgewichtsmodell im Vergleich zur Wellenlänge der Störung nur wenig ändert. Insofern die oszillatorischen Störungen nachfolgend dargestellte Form haben:

$$
\xi_{r} \sim \rho_{0}^{-1 / 2}(r) e^{i k_{r} r}, \quad P_{1} \sim \rho_{0}^{-1 / 2}(r) e^{i k_{r} r}
$$

ergibt sich im Falle einer geschichteten isothermen Atmosphäre folgende Dispersionsrelation ([92]):

$$
k_{r}^{2}=\frac{\omega^{2}-\omega_{A}^{2}}{c^{2}}+S_{l}^{2} \frac{N^{2}-\omega^{2}}{c^{2} \omega^{2}}
$$

mit den Hilfsgrößen 


$$
\begin{aligned}
S_{l}^{2} & =\frac{l(l+1)}{r^{2}} c^{2} & & (\text { Lamb }-- \text { Frequenz) } \\
\omega_{A} & =\frac{c}{2 H} & & \text { (akustische Cutoff-Frequenz) } \\
H & =\frac{\rho_{0}}{\frac{d \rho_{0}}{d r}}=\left(\frac{g}{c^{2}}+\frac{N^{2}}{g}\right)^{-1} & & \text { (Dichteskalenhöhe) } \\
k_{h}^{2} & =\frac{l(l+1)}{r^{2}} & &
\end{aligned}
$$

$g$ und $N$ repräsentieren die Schwerebeschleunigung und die Brunt-Väisälä-Frequenz und haben folgende Formen:

$$
\begin{array}{r}
g=1 / \rho_{0}\left(d P_{0} / d r\right) \\
N^{2}=g\left(\frac{1}{\Gamma_{1} P_{0}} \frac{d P_{0}}{d r}-\frac{1}{\rho_{0}} \frac{d \rho_{0}}{d r}\right)
\end{array}
$$

Der radiale und der horizontale Anteil des Wellenvektors wird von $k_{r}$ und $k_{h}$ beschrieben. Als Grenzfälle ergeben sich aus Gl. (6.12) die Dispersionsrelationen für reine Schall- und reine Schwerewellen ([92]):

$$
\begin{array}{ll}
k^{2}=k_{r}^{2}+k_{h}^{2}=\frac{\omega^{2}}{c} \quad \text { für } \quad \omega \gg \omega_{A} \quad \text { (Schallwellen), } \\
k_{r}^{2}=\frac{S_{l}^{2}}{c^{2}}\left(\frac{N^{2}}{\omega^{2}}-1\right) & \text { für } \quad \omega \ll \omega_{A}, N \quad \text { (Schwerewellen). }
\end{array}
$$

Für große $l$ folgt daraus:

$$
\omega^{2}=N \frac{k_{h}^{2}}{k_{r}^{2}+k_{h}^{2}} \equiv N^{2} \sin ^{2} \Theta,
$$

wobei der Winkel $\Theta$ der Winkel zwischen der Vertikalen und der Ausbreitungsrichtung der Welle ist. In Bereichen mit $k_{r}^{2}>0$ existieren oszillatorische Lösungen, in Bereichen mit $k_{r}^{2}<0$ sind die Lösungen exponentiell ansteigend oder abfallend. Die konvektive Instabilität tritt für $N^{2}<0$ ein. Abbildung 6.1 stellt diese Bereiche in einem sogenannten diagnostischen Diagramm dar.

Diese mehr oder weniger groben Betrachtungen haben folgende Konsequenzen für die solaren Oszillationen:

- Die beobachteten 5-min-Oszillationen sind aufgrund der Wellenzahlen, des Grades $l$ und der Frequenz den solaren p-Moden zugeordnet.

- Die Oszillationen sind in der solaren Atmosphäre, welche eine akustische Cutoff-Frequenz von $5.2 \mathrm{mHz}$ besitzt, evaneszent. 


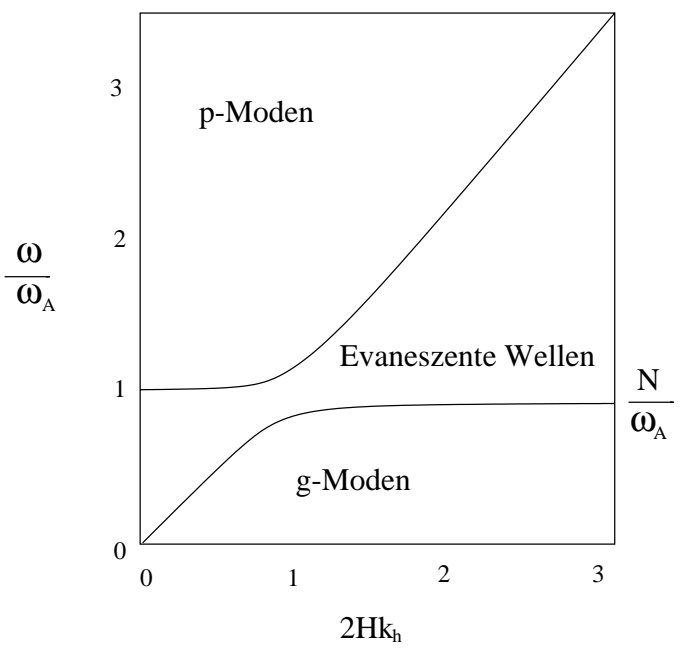

Abbildung 6.1: Das diagnostisches Diagramm zeigt die Frequenzregionen in denen die Lösungen, welche der Dispersionsrelation Gl. (6.12) entsprechen, liegen. Die durchgezogenen Linien entsprechen $k_{r}=0$. In den $p-M o d e n-$ und g-Moden-Bereichen sind die Lösungen räumlich oszillatorisch. Im evaneszenten Bereich sind sie exponentiell abfallend.

- Die solare Atmosphäre repräsentiert den oberen Deckel einer Kavität, in der sich aus einwärts und auswärtslaufenden Wellen stehende Wellen ausbilden können. Der untere Reflexionspunkt $r_{t}$ folgt aus der Dispersionsrelation Gl. (6.12) für $k_{r}^{2}=0$ :

$$
r_{t}=[l(l+1)]^{1 / 2} \frac{c\left(r_{t}\right)}{\omega}
$$

Abb. 6.2 zeigt seine Abhängigkeit von dem Grad $l$.

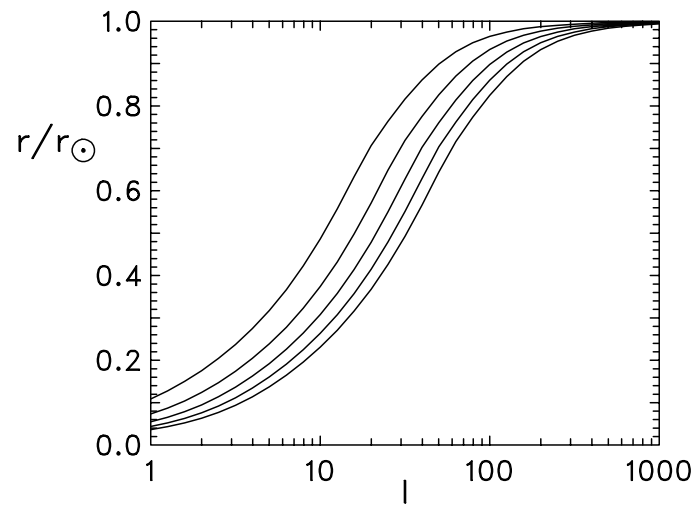

Abbildung 6.2: Tiefe des inneren Reflektionspunkt in Einheiten des Sonnenradius im Falle von $\frac{\omega}{2 \pi}=1.5,2.25,3,3.75$ und $4.5 \mathrm{mHz}$ ([92], S.168)

Abb. 6.3 stellt den Ausbreitungsweg zweier p-Moden mit großem und kleinem $l$ schematisch dar. Nahe an der Sonnenoberfläche verschwindet die Horizontalkomponente des oszillatorischen Geschwindigkeitsfeld. Sobald jedoch eine leichte Neigung des Wellenvektors vorliegt nimmt diese mit zunehmender Tiefe hin zu, was 
damit zu erklären ist, daß der Teil einer Wellenfront, der in tieferen, heißeren atmosphärischen Bereichen lokalisiert ist, sich schneller ausbreitet. In einer bestimmten Tiefe besitzt der Wellenvektor nur noch eine horizontale Komponente und die Wellenfront wird faktisch nach oben reflektiert. Für große $l$ liegt der untere Reflektionspunkt nahe der Sonnenoberfläche, während er für kleine $l$ in tieferen Regionen liegt. Im Zusammspiel beider Reflektionspunkte wandern solche p-Moden um die Sonne.

Deubner führte 1975 Beobachtungen der ruhigen Sonne für die solare 5-Minuten-

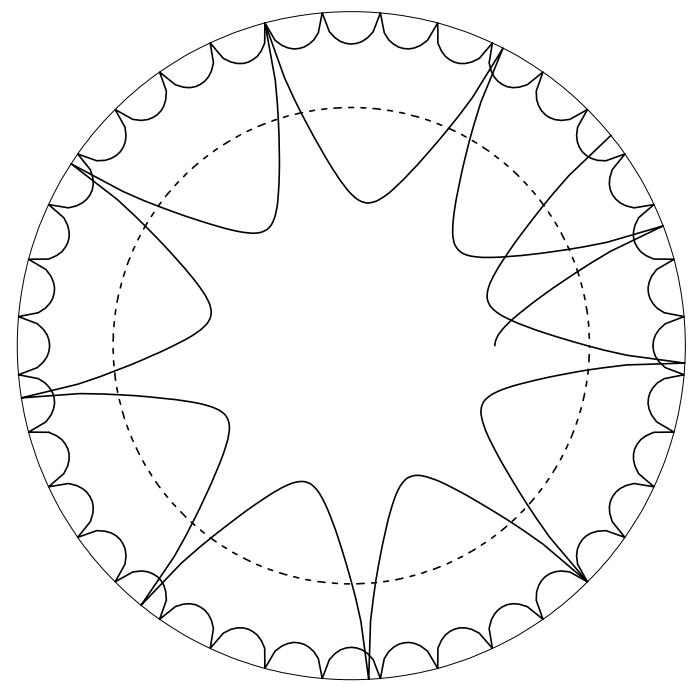

Abbildung 6.3: Schematische Darstellung des Ausbreitungswegs von zwei p-Moden mit großem und kleinem $l$, die um die Sonne wandern ([92], S.168)

Oszillationen $(3.3 \mathrm{mHz})$ in hoher räumlicher und zeitlicher Auflösung für $l \geq$ 150 durch ([18]). Diese unterzog er einer Power- oder Frequenzanalyse. Zu einer Fourier-oder Poweranalyse lässt sich ganz allgemein sagen, daß die relative Stärke eines Schwingungsmodes durch das Frequenzleistungs- oder Powerspektrum beschrieben wird. Dabei ist $F(k)$ die Fouriertransformierte einer Funktion $f(x, t)$ des Ortsraums, welche im aktuellen Fall die Geschwindigkeitsfluktuationen darstellt. $F(k)$ stellt eine Entwicklung nach orthonormalen Eigenfunktionen, den trigonometrischen Funktionen, dar und wird durch eine Transformation vom Orts-in den Frequenzraum vollzogen. Eine solche Entwicklung läßt sich sowohl für räumlichen als auch für zeitlichen Variationen durchführen (im weiteren beschreibt $k$ allgemein die Frequenz einer Schwingung). Der Betrag von $|F(k)|$ ist die Amplitude einer Schwingung mit der Frequenz $k$. Das Betragsquadrat $|F(k)|^{2}$ der Fouriertransformierten $F(k)$ wird als Frequenzleistungsspektrum oder Powerspektrum $P(k)$ bezeichnet ([92], [66], [3]).

Deubner bestimmte die Abhängigkeit des Powerspektrums von der Frequenz $\omega$ und dem Grad $l$. Die Resultate, in einem $k-\omega$-Diagramm dargestellt, zeigen, daß sich die Power entlang diskreter Linien konzentriert, welche mit den Frequenzen, der solaren akustischen Eigenschwingungen, übereinstimmen (Abb. 6.4). Damit konnten die von Ulrich 1970 ([99]) theoretisch vorhergesagten Frequenzintervalle der 
p-mode-ridges erstmals experimentell nachgewiesen werden. Mittels verschiedener Beobachtungen ([7], [22], [24], [23]) wurde für einen Frequenzbereich von $1.4 \mathrm{mHz} \leq \nu \leq 4.2 \mathrm{mHz}$ und einen $l$-Bereich $l \geq 200$ die Power bestimmt und die Ergebnisse von Deubner ([18]) bestätigt und auf einen größeren Frequenz- und l-Bereich ausgedehnt. Arbeiten von Deubner ([19])s zeigen, daß zwischen den ' $p$ mode-ridges' ein kontinuierliches kohärentes Geschwindigkeitsfeld existiert und , daß das photosphärische Geschwindigkeitsfeld schon für den Fall der ruhigen Sonne sehr komplex ist. Neueste Langzeitbeobachtungen, die entweder auf einer in

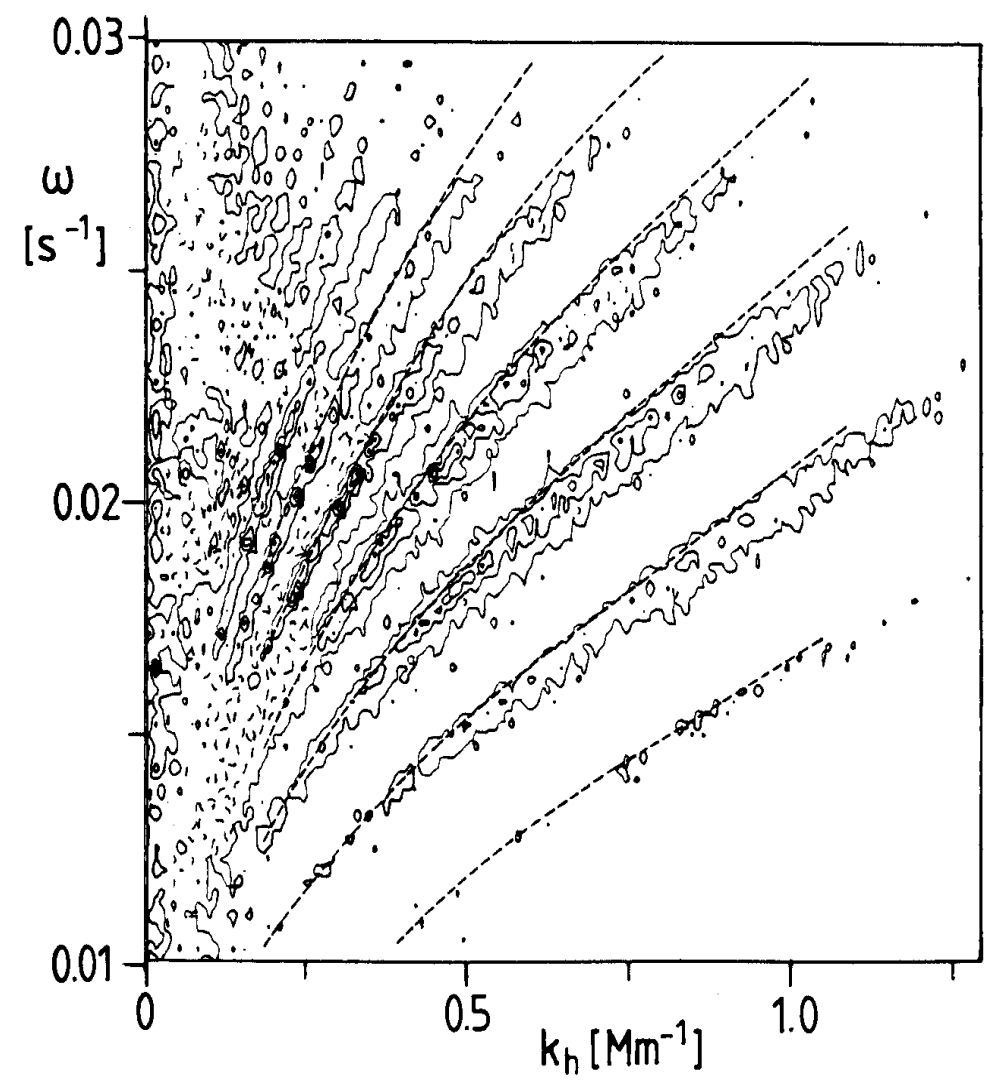

Abbildung 6.4: $p$-mode-ridges in der $k_{h}, \omega$ Ebene. Die Isolinien stellen das aus Beobachtungen gewonnene Power ([18]) dar, während die gestrichenen Linien die dazu korrespondierenden Resultate aus einem theoretischen Sonnenmodel sind ([92], S.154)

großer geographischer Breite lokalisierten Meßstation, während des polaren Sommers oder mittels eines Netzwerks aus Beobachtungsstationen [Gong (Harvey et al. 1996), Bison (Chaplin et al. 1996a), Iris (Fossat 1991), Ton (Chou et al. 1995), [50], [6],[27]], oder aber mittels des Michelson-Doppler-Imager (MDI) des Satelitten SOHO gemacht wurden ([20]), liefern zeitlich hochaufgelöste Zeitreihen. Die korrespondierenden $k-\omega$-Diagramme solcher Beobachtungen zeigt mehr als 30 solcher $p$-mode-ridges. Der zugehörige Frequenzbereich liegt zwischen 1. und $8 \mathrm{mHz}$. Der Grad $l$ überdeckt den Bereich von $l=5, \ldots, 250$. Der Vergleich zu den von Deubner (1979) ermittelten Resultaten $(l=70, \ldots, 1000)$ zeigt eine deutliche Erweiterung hin zu kleinen $l$.

Kohärente Schwingungsmuster mit Perioden von einigen Minuten wurden auch in numerischen Simulationen der solaren Konvektion gefunden. Dabei wurden von 
Stein et al. (1988) und Stein \& Nordlund (1988) starke Schwingungen in Frequenzbereichen gefunden, die den Eigenschwingungen des Modellgebiets entsprechen (Stein et al. (1990); [89]). Steffen et al. (1991) fanden starke Schwingungsbeiträge in Frequenzbereichen, die nahe der akustischen cut-off Frequenz der Atmosphäre $(5-7) \mathrm{mHz}([88])$ liegen. 


\subsection{Schwingungen kleinskaliger Magnetfeldkonzen- trationen}

Da die direkte Beobachtung von kleinräumigen magnetischen Strukturen durch das Auflösungsvermögen der optischen Instrumente im Zusammenspiel mit den atmosphärischen Brechungsindexfluktuationen begrenzt ist ([77], [92]), lassen sich bislang nur durch Einsatz indirekter Diagnosetechniken Erkenntnisse über deren thermodynamische Strukturierung gewinnen ([92]). Um Aufschlüsse über die oszillatorischen Geschwindigkeitsmuster in diesen kleinräumigen Magnetfeldkonzentrationen zu erzielen, führten Giovanelli et al. (1978), Wiehr (1985), Solanki (1986), Volkmer et al. (1995) ([32], [31], [33], [107], [79], [80], [103]) spektropolarimetrische Beobachtungen durch. Die von Giovanelli et al. gezeigten Resultate, wurden durch simultane Beobachtungen in zwei Spektralinienzentren eines einzigen Punktes in der Sonnenscheibenmitte gewonnen. Dabei wurden zwei verschiedene Linienpaare untersucht. Das eine Paar setzte sich aus zwei Fe-I-Absorptionslinen bei $5250 \AA$ und $5233 \AA$ zusammen. Das andere bestand aus der Fe-I- und der Mg-I-Absorptionsline bei $5166 \AA$ und $5183 \AA$. Zusätzlich wurde parallel dazu in $H_{\alpha} \quad(\lambda=6563 \AA)$ beoachtet. Die photosphärischen Fe-I-Linien bei $5250 \AA$ und bei $5233 \AA$ entstehen annährend in derselben atmosphärischen Tiefe, so daß die Dopplerverschiebungen der Linienkerne, bewirkt durch nach außen wandernde Wellen, etwa zur selben Zeit wahrnehmbar waren. In der chromosphärischen Mg I 5183 Å Linie tauchten sie 19 sec später auf. Die typischen Geschwindigkeitsamplituden wuchsen mit zunehmender atmosphärischer Höhe an, so betrugen sie etwa $\pm 0.2 \mathrm{~km} / \mathrm{s}$ in der photosphärischen Fe-I-Linie bei $5166 \AA, \pm 0.4 \mathrm{~km} / \mathrm{s}$ in der chromosphärischen Mg-I-Line (5183 $\AA$ ) und $\pm 0.7 \mathrm{~km} / \mathrm{s}$ in $H_{\alpha}$, der Spektrallinie mit der größten Entstehungshöhe. Die Perioden betrugen etwa 5 min in den Fe-I-Linien und der Mg-I-Linie, allerdings nur noch 3 min in $H_{\alpha}$. Die starke Kopplung der Schwingungsmuster innerhalb und außerhalb der Magnetfeldkonzentrationen deuten entweder auf eine starke Wechselwirkung dieser Bereiche hin oder auf denselben Anregungsmechanismus. Giovanelli et al. schlossen daraus auf sich ausbreitende Wellen, welche eine Phasengeschwindigkeit von $100 \mathrm{~km} / \mathrm{s}$ besitzen. Roberts (1983)([72]) verglich diese Resultate mit theoretischen Berechnungen, die er unter Annahme der Näherung dünner Flußröhren und durch Linearisierung der Gleichungen erhielt ([68]). Dabei bedeutet dünn, daß der Flußröhrenradius viel geringer als die Wellenlänge der Flußröhrenwelle ist. Er identifizierte die von Giovanelli gefundenen kohärenten Geschwindigkeitsmuster mit longitudinalen Wellen, den sogenannten sausage-modes. Giovanelli et al. (1978) und Wiehr (1985) ([32], [107]) fanden, daß oszillatorische Schwingungsmuster innerhalb der magnetischen Flußröhren im großen und ganzen in Phase mit denen der nichtmagnetischen Umgebung zu sein scheinen. Diese starke Kopplung von externem und internem Schwingungsverhalten legt die Vermutung eines großskaligen Hintergrundsmuster bestehende aus den 5-min-Oszillationen nahe. Die von Volkmer et al. (1995) ([103]) mittels spektropolarimetrischer Beobachtungen gewonnenen Resultate zeigen zusätzlich zu den 5 min Oszillationen auch Schwingungsmuster in einem Frequenzbereich von $6-10 \mathrm{mHz}$, jenseits der akustischen Cutoff-Frequenz der Atmosphäre, welche 
von den sausage-modes der Magnetfeldkonzentrationen herrühren könnten ([68]). Abb. 6.5 stellt das entsprechende Powerspektrum der vertikale Geschwindigkeitskomponente dar. Durch theoretische Untersuchungen von Defouw (1976), Roberts

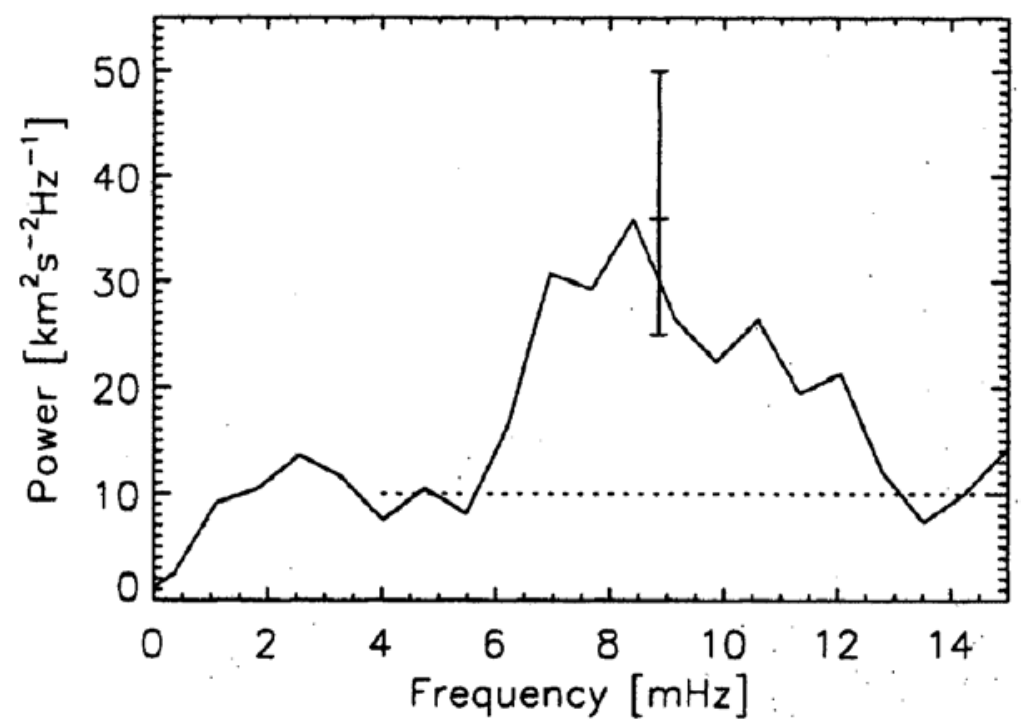

Abbildung 6.5: Powerspektrum der zeitlichen Entwicklung der SichtlinienGeschwindigkeitskomponente innerhalb einer Flußröhre [Volkmer et al. ([103])].

\& Webb (1978, 1979), Spruit (1981 a,b), Edwin \& Roberts (1982) ([16], [73], [74] [87], [86] [25]) ist das Schwingungsverhalten von isolierten Flußröhren im Falle eines ungeschichteten Mediums gut verstanden. Die grundsätzlichen Muster dieser Schwingungen, welche von den Magnetfeldkonzentrationen beschrieben und im weiteren als Flußröhrenwellen bezeichnet werden, sind in Abb. 6.6 dargestellt. Das Schwingungsverhalten von isolierten zweidimensionalen Flußschichten eingebettet in ungeschichteten Atmosphären wurde von Cram \& Wilson (1975), Roberts (1981b) und von Edwin \& Roberts (1982) ([9], [71], [25] theoretisch studiert. Alle diese Untersuchungen basieren auf linerarisierten Störungsrechnungen für ein ideales Plasma in der sich die Störungen adiabatisch ausbreiten. Im Fall einer magnetischen Flußröhre in einer gravitativ geschichteten Atmosphäre beschränken sich die theoretischen Untersuchungen auf dünne Flußröhren. Roberts \& Webb (1978, 1980) ([73], [74], [76], [75]) studierten das Schwingungsverhalten einer isolierten magnetischen Flußröhre in einer gravitativ geschichteten Atmosphäre anhand von linearen Analysen, auch unter Berücksichtigung der Strahlungsdämpfung. Das Magnetfeld besitzt in diesem Fall nur eine Vertikalkomponente, ist homogen innerhalb der Röhre und variiert in vertikaler Richtung. Das System der zeitabhängigen MHD-Gleichung für die dünnen Flußröhren hat nachfolgend dargestellte Form 

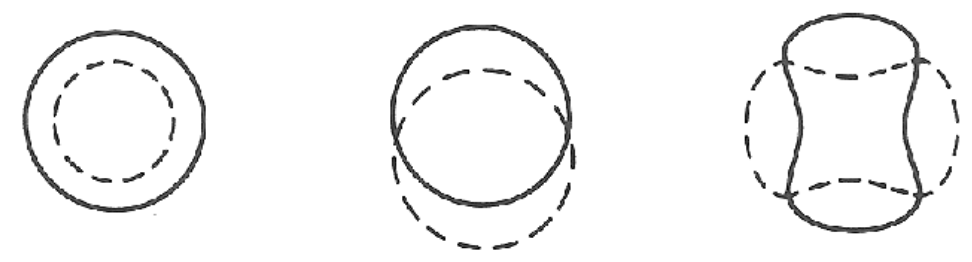

$n=0$

$n=1$

$n=2$
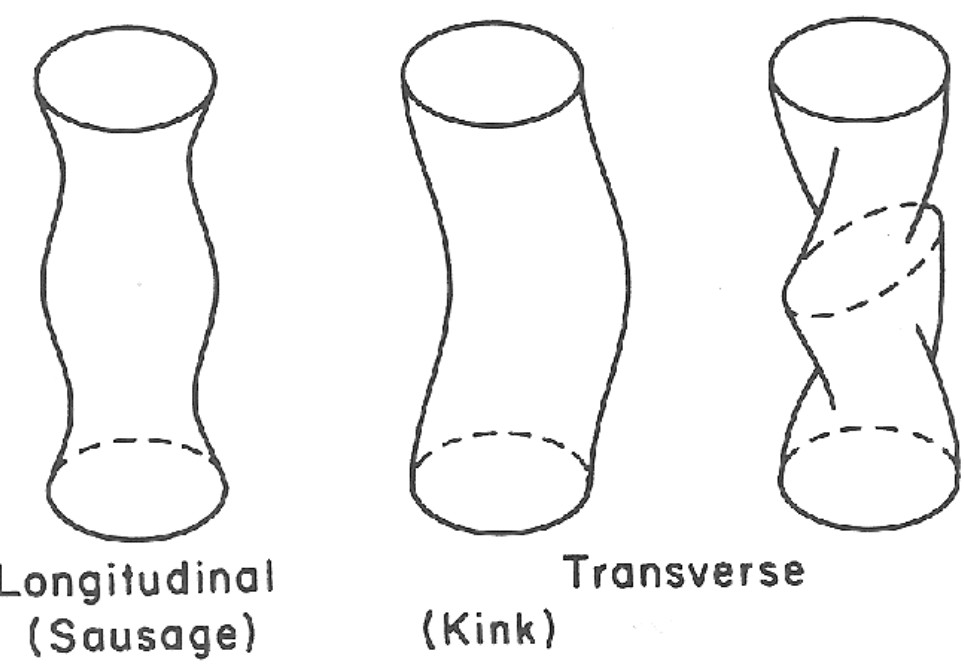

Transverse

(Kink)

Abbildung 6.6: Wellentypen einer zylinderförmigen Flußröhre ([87])

([16], [73], [84]):

$$
\begin{array}{r}
\rho\left(\frac{\partial \vec{v}}{\partial t}+\nabla \cdot\left(\rho \vec{v} \vec{v}^{T}\right)\right)=-\frac{\partial p}{\partial z}-\rho g \\
\frac{\partial}{\partial t}\left(\frac{\rho}{B}\right)+\frac{\partial}{\partial z}\left(\frac{\rho \vec{v}}{B}\right)=0 \\
p_{e}(z)=p_{i}(z)+\frac{B^{2}(z)}{8 \pi}
\end{array}
$$

( $e \equiv$ extern, $i \equiv$ intern, $H_{p}$ : Druckskalenhöhe, $p_{e}$ : Gasdruck im äußeren Gebiet, $p_{i}$ : Gasdruck in der Flußröhre, $B:$ vertikale Magnetfeldkomponente).

Dabei ergibt sich unter der Annahme konstanter Temperatur $T_{e}=T_{i}=$ const der Druck zu $p_{e}(z)=p_{0} \exp \left(-z / H_{p}\right)$. Gl. (6.18) sich aus der Kombination von Kontinuitäts- und Induktionsgleichung zusammen.

Die Alfvéngeschwindigkeit $v_{A}=\left(B^{2} / 4 \pi \rho\right)^{1 / 2}$ und $\beta=8 \pi p / B^{2}$ sind aufgrund der gemachten Annahmen höhenunabhängig und mit $A \cdot B=$ const ( $A$ : Querschnittsfläche der Flußröhre) folgt für die ungestörte Röhre:

$$
p \propto \rho \propto B^{2} \propto \exp \left(-z / H_{p}\right), \quad A \propto B^{-1} \propto \exp \left(-z / 2 H_{p}\right)
$$


Die linearisierte Form der Gleichungen Gl. (6.17) - Gl. (6.19) läßt sich im Falle adiabatischer Störungen, unter Vernachlässigung der Strahlungsdämpfung und mit der Annahme das $\vec{v}=\left(0,0, v_{z}\right)$ ist (longitudinale Wellen), zu einer Gleichung für die Geschwindigkeitsstörung zusammenfassen, die eine Lösung der Form $v(z, t)=v_{0} \exp \left(-z / 4 H_{p}+i k z+i \omega t\right)$ hat.

Die Dispersionsrelation hat folgende Form:

$$
\omega^{2}=c_{T}^{2} k^{2}+\frac{c_{T}^{2}}{H_{p}^{2}}\left[\frac{c_{S}^{2}}{v_{A}^{2}}\left(\frac{\gamma-1}{\gamma^{2}}\right)+\frac{9}{16}-\frac{1}{2 \gamma}\right] .
$$

Für die sogenannte tube speed gilt:

$$
c_{T}^{2}=c_{S}^{2} v_{A}^{2} /\left(c_{S}^{2}+v_{A}^{2}\right)=\text { const }
$$

( $c_{S}$ : Schallgeschwindigkeit, $\gamma=c_{p} / c_{v}$ : Adiabatenexponent).

Im Falle einer endlichen Druckskalenhöhe ist eine vertikale Ausbreitung longitudinaler Flußröhrenwellen $\left(k^{2}>0\right)$ nur für Frequenzen möglich, die größer als die sogenannte Cutoff-Frequenz $\omega_{c 1}$ sind, welche erstmals von Defouw (1976) ([16]) abgeleitet wurde:

$$
\omega_{c 1}=\left(\frac{c_{T}^{2}}{H_{p}^{2}}\left[\frac{c_{S}^{2}}{v_{A}^{2}}\left(\frac{\gamma-1}{\gamma^{2}}\right)+\frac{9}{16}-\frac{1}{2 \gamma}\right]\right)^{1 / 2} .
$$

Unter Einbeziehung der Strahlungsdämpfung bekommt die Dispersionsrelation folgende Gestalt ([76], [75]):

$$
\begin{aligned}
\omega_{c 2}= & \left\{-\frac{1}{2}\left[\frac{1}{\gamma^{2} \tau_{R}^{2}}\left(1+(\gamma-1) \frac{c_{T}^{2}}{c_{S}^{2}}\right)-\omega_{c 1}^{2}\right]\right. \\
& \left.\cdot\left(\frac{1}{4} \cdot\left(\frac{1}{\gamma^{2} \tau_{R}^{2}}\left(1+(\gamma-1) \frac{c_{T}^{2}}{c_{S}^{2}}\right)-\omega_{c 1}^{2}\right)^{2}-\frac{c_{T}^{2}}{\gamma^{2} \tau_{R}^{2} 16 H_{p}^{2}}\right)^{1 / 2}\right\}^{1 / 2}
\end{aligned}
$$

Dabei erfolgt der Strahlungsenergieaustausch zwischen Flußröhre und ihrer Umgebung entsprechend dem Newtonschen Abkühlungsgesetz, d.h. proportional zur Temperaturdifferenz zwischen Röhre und Umgebung, innerhalb der von Spiegel \& Unno (1966) ([81], [100]) abgeleiteten Strahlungsrelaxationszeit:

$$
\tau_{R}=\frac{c_{v}}{16 \kappa_{R} \sigma T^{3}}\left[1-\frac{\kappa_{R} \rho}{d} \cot ^{-1}\left(\kappa_{R} \rho d\right)\right]^{-1}
$$

$\left(\kappa_{R}\right.$ : Rosselandsche Absorptionskoeffizient Gl. (4.4), $d$ : Flußröhrendurchmesser, $\sigma$ : Stefan-Boltzmann-Konstante, $c_{v}$ : spezifische Wärmekapazität).

Für die transversalen Röhrenwellen (kink-modes) einer dünnen Flußröhre, die in einer isothermen Atmosphäre eingebettet ist, hat Spruit (1981) ([87], [86]) eine Dispersionsrelation abgeleitet. Dabei lag die Annahme zu Grunde, daß die Flußröhre sich im Temperaturgleichgewicht mit ihrer Umgebung befindet und die transversale 
Verschiebung $\xi$ der Röhre folgender Bewegungsgleichung gehorcht:

$$
(2 \beta+1) \frac{\partial^{2} \xi}{\partial t^{2}}=g \frac{\partial \xi}{\partial z}+2 g H_{p} \frac{\partial^{2} \xi}{\partial z^{2}}
$$

Diese Gleichung hat Lösungen der Form $\xi=\xi_{0} \exp \left(i \omega t+i k z-z / 4 H_{p}\right)$ mit der Dispersionrelation:

$$
\omega^{2}=c_{k}^{2} k^{2}+\frac{g}{8 H_{p}(2 \beta+1)} .
$$

$\beta=8 \pi p / B^{2}=$ const beschreibt das Verhältnis von kinetischem Gasdruck $p$ und dem magnetischem Druck. Die Geschwindigkeit einer solchen transversalen-Welle (kink-Welle) ist die kink-Geschwindigkeit:

$$
c_{k}^{2}=\beta v_{A}^{2} /(2 \beta+1)=\rho v_{A}^{2} /\left(\rho_{e}+\rho_{i}\right) .
$$

Eine Ausbreitung der kink-Wellen ist nur für Frequenzen oberhalb der cutoff-Frequenz $\omega_{c 3}$ möglich:

$$
\omega_{c 3}=\left(\frac{g}{8 H_{p}(2 \beta+1)}\right)^{1 / 2} .
$$

Grundsätzlich entspricht die Annahme einer dünnen Flußröhre in den oberen Bereichen der Photosphäre und in der Chromosphäre nicht den realen Verhältnissen. Da dort die Dichte- und Druck-Skalenhöhen sehr gering sind, ist die Bedingung, daß der Flußröhrenradius viel kleiner als die lokale Druckskalenhöhe $H_{p}\left(r \ll 4 H_{p}\right)$ ist, nicht erfüllt. Des Weiteren wird die Krümmung vernachlässigt obwohl diese in diesen Bereichen stark zunimmt, da sich die Flußröhren sehr stark aufweiten. Somit variieren das Magnetfeld und die atmosphärischen Bedingungen stark mit dem Abstand $r$ von der Flußröhrenachse und der atmosphärischen Höhe $z$, so daß der Linearisierungsansatz nur in beschränktem Maße anwendbar ist.

\subsection{Schwingungsanalyse numerischer Simulationen}

Im Folgenden werden drei zeitabhängige Simulationen kleinskaliger Magnetfeldkonzentrationen anhand von Fourieranalysen (Poweranalysen) ([3]), die in Kap. 6.1 einführend erläutert werden, auf ihr typisches Schwingungsverhalten untersucht und die Resultate mit dem linearen Ansatz verglichen. Auf der einen Seite wurden die Resultate aus zwei 2-D-MHD-Simulationen [Steiner et al. (1998) ([90])] (im folgenden mit tube und pore bezeichnet) einer solchen Analyse unterzogen und dienten zum Vergleich mit den Resultaten der von mir mit dem WDV-Code durchgeführten Simulation. Die beide Simulationen unterscheiden sich im magnetischen Fluß und der simulierten Zeit. Die Randbedingungen und die numerischen Methoden sind in Tabelle 6.1 kurz und in Steiner et al. ([90], [91]) ausführlich erläutert. Die Anfangslösungen in den Simulationsläufen I und II bestehen aus einem plan- 
parallelen Photosphärenmodell ([83]) in welches eine magnetische Flußschicht eingebettet ist. Diese ist im Fall tube beim Niveau $\tau_{5000}=1$ etwa $300 \mathrm{~km}$ breit, hat eine maximale Magnetfeldstärke von etwa $1 \mathrm{kG}$ und befindet sich im horizontalen Druckgleichgewicht. Simuliert wurden 40 min reale Zeit. Die Anfangslösung im Simulationslauf II unterschied sich von der des tube-Run in der Breite der magnetischen Flußschicht, die bei $\tau_{5000}=1$ etwa $800 \mathrm{~km}$ beträgt und eine maximale Feldstärke von etwa $3 \mathrm{kG}$ hat. In diesem Fall betrug die simulierte Zeitspanne etwa 45 min. Abb. 6.7 und Abb. 6.8 zeigen das Geschwindigkeitsfeld und die Magnetfeldlinien zu verschiedenen Zeitpunkten für den tube- und den pore-Run. In allen drei Fällen betrug die zeitliche Auflösung 1 Sekunde, was einer maximal auflösbaren Frequenz, der Nyquistfrequenz, von $250 \mathrm{mHz}$ entspricht.

Nachfolgend sind die Unterschiede zwischen beiden 2-D-MHD-Codes (WDVCode und ST-Code) und die verwendeten Anfangs-und Randbedingungen in Tabelle 6.1 aufgelistet:

\section{Vergleich der 2-D-MHD-Codes und der Konfigurationen}

\begin{tabular}{|c|c|c|}
\hline Charakteristika & WDV-Code & ST-Code \\
\hline \multirow[t]{2}{*}{$\begin{array}{c}\text { Gitterzellen- } \\
\text { geometrie }\end{array}$} & unstrukturiertes Dreiecksgitter & kartesisches Gitter \\
\hline & lokale Gitteradaption & statisches Gitter \\
\hline \multicolumn{3}{|l|}{ Rechengebiet } \\
\hline & horizontal $[\mathrm{km}]$ & horizontal $[\mathrm{km}]$ \\
\hline & 12000 & 2400 \\
\hline & vertikal $[\mathrm{km}]$ & vertikal $[\mathrm{km}]$ \\
\hline & 1200 & 1400 \\
\hline \multicolumn{3}{|l|}{ Randbedingungen } \\
\hline seitlich & periodisch & periodisch \\
\hline oben & geschlossen, $\frac{\partial T}{\partial z}=0$ & geschlossen, $\frac{\partial T}{\partial z}=0$ \\
\hline unten & offen & geschlossen \\
\hline Anfangsbedingungen & $\begin{array}{c}\text { homogenes } \vec{B}=\left(0,0, B_{z}\right) \\
\text { in vollentwickelte hydro- } \\
\text { dynamische }(\mathrm{HD}) \text { Konvektion }\end{array}$ & $\begin{array}{l}\text { magn. Flußschicht in } \\
\text { HD-Konvektion }\end{array}$ \\
\hline Strahlungstransport & grau, ESC1/2 (Kap. 4) & $\begin{array}{c}\text { grau, } \\
\text { 'short-charakteristics' (Kap. 4) }\end{array}$ \\
\hline
\end{tabular}

Tabelle 6.1: Vergleich der beiden 2-D-MHD-Codes (WDV-Code und ST-Code) und den benutzten Anfangs-und Randbedingungen und Strahlungstransportlöser. 

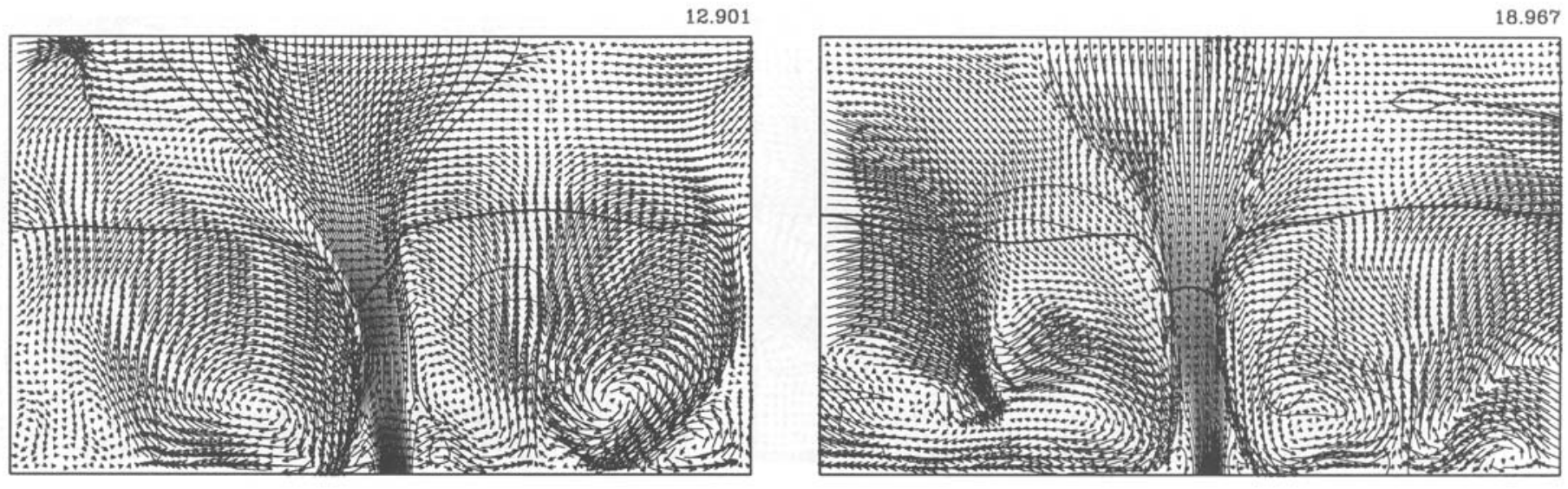

14.934
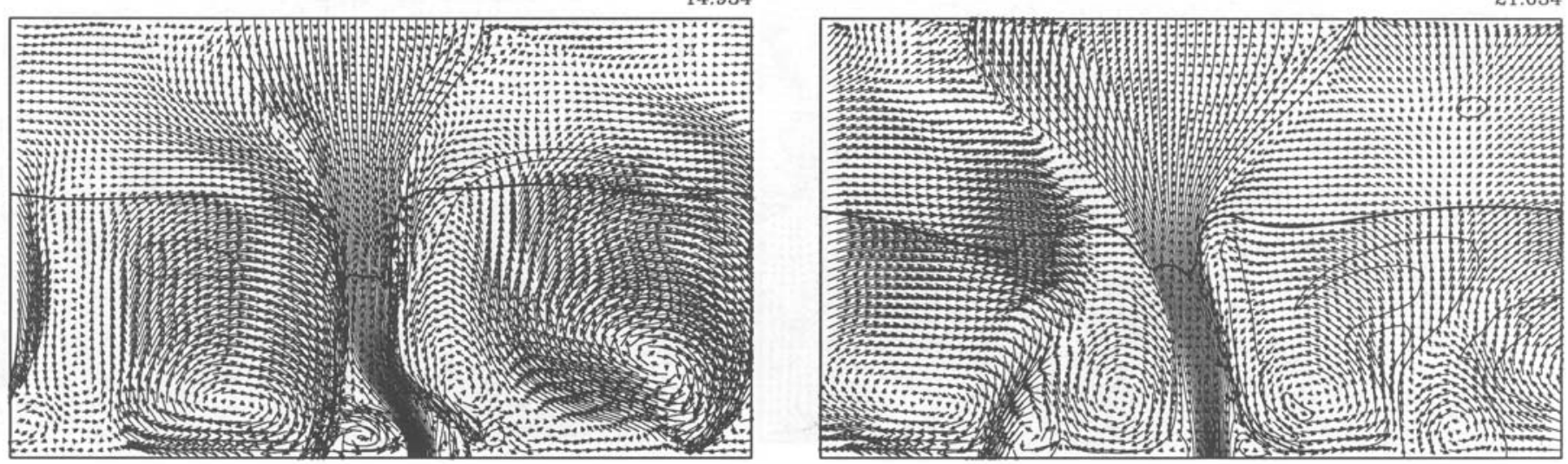

17.134
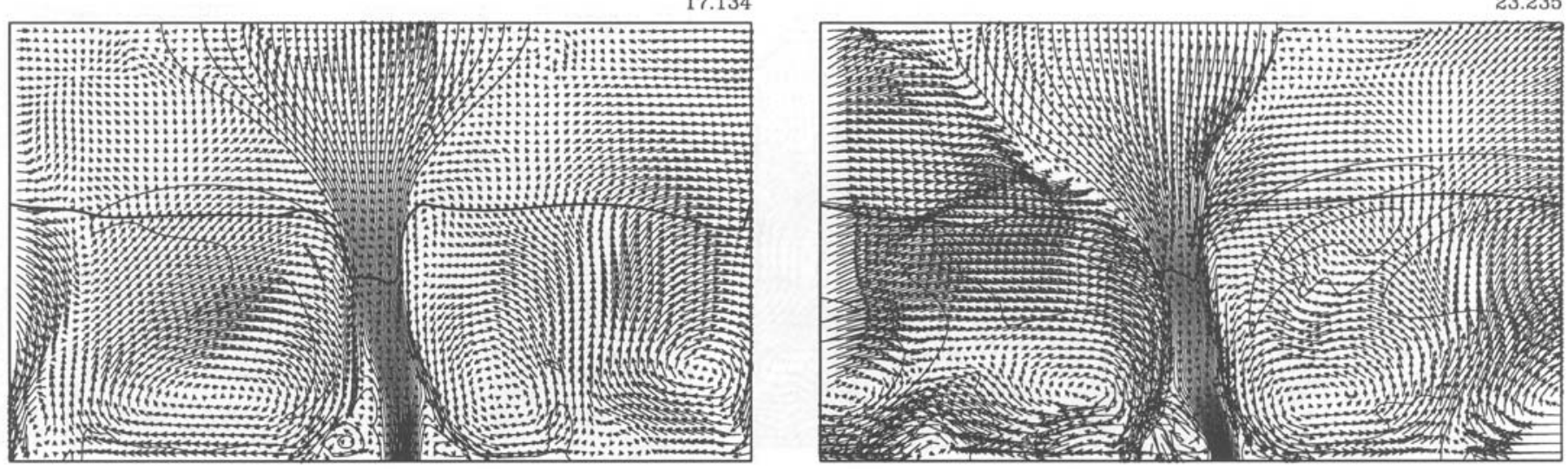

Abbildung 6.7: Magnetfeldlinien und Geschwindigkeitsfeld (Vektorpfeildarstellung) verschiedener Zeitpunkte des tube-Run. Die Zeitpunkte sind in der oberen rechten Bildecke in entdimensionierten Einheiten angezeigt und entsprechen 40.8, 47.2, 54.2, 60, 66.6 und 73.6 Minuten. 
31.120

39.110
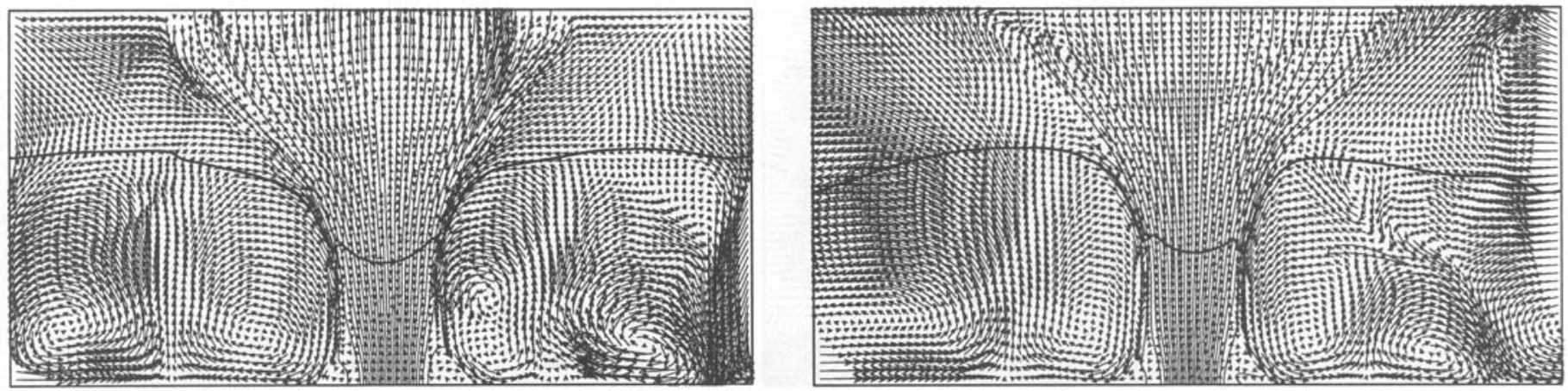

33.739

41.675
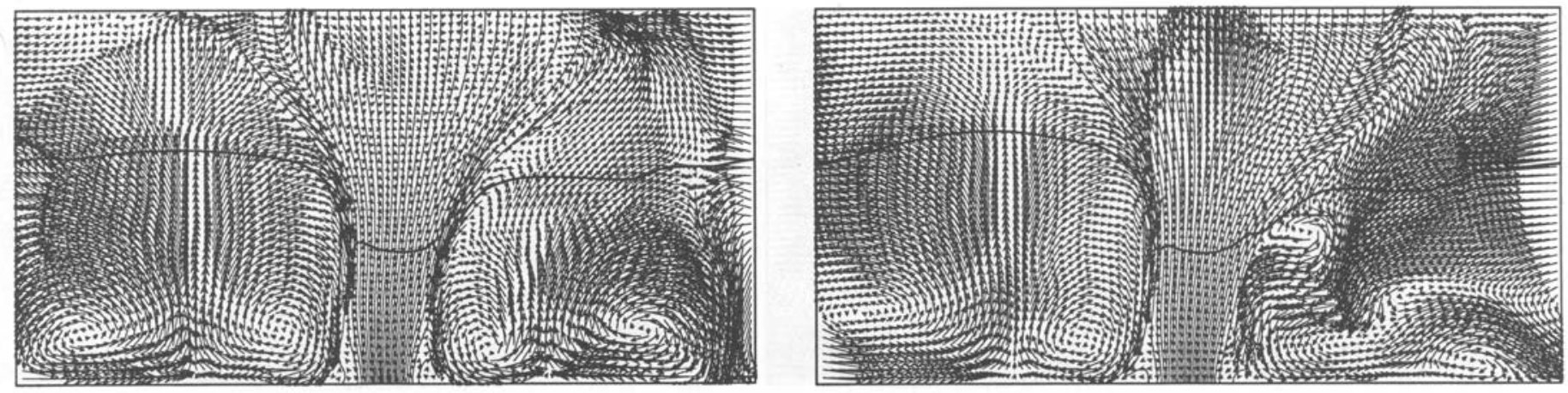

36.463
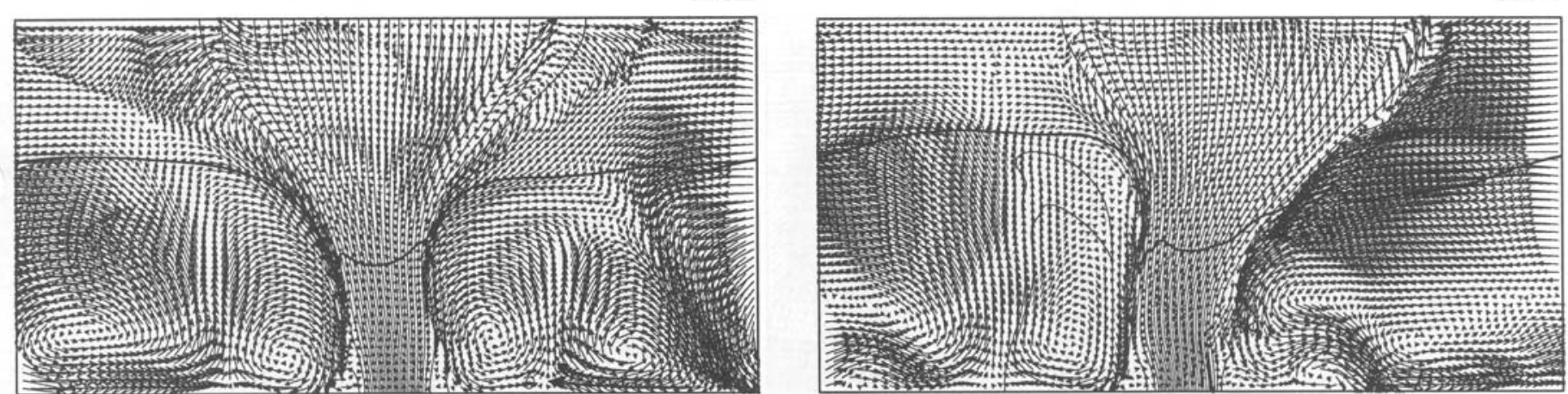

Abbildung 6.8: Magnetfeldlinien und Geschwindigkeitsfeld (Vektorpfeildarstellung) verschiedener Zeitpunkte des pore-Run. Die Zeitpunkte sind in der oberen rechten Bildecke in entdimensionierten Einheiten angezeigt und entsprechen 98.5, 106.8, 115.4, 123.8, 131.9 und 139.8 Minuten. 


\subsubsection{Fourier- oder Poweranalyse der Resultate des ST-Code}

In den beiden untersuchten Simulationen sind innerhalb und außerhalb der Magnetfeldkonzentrationen, wie in den Abbildungen 6.9 und 6.10 gezeigt, Schwingungsmuster zu sehen. Daher wurden die Simulationsresultate einer Poweranalyse unterzogen. Zu diesem Zweck ist das Rechengebiet in ein magnetisches und ein schwachmagnetisches Teilgebiet aufgeteilt worden. Als magnetisch ist in jeder Höhe $z$ der Teilbereich bezeichnet worden in dem $B_{z}>500$ Gauß ist. Als schwachmagnetisch derjenige in dem $B_{z}<500$ Gauß war. In den Teilgebieten sind dann zu jedem Zeitpunkt horizontale Mittelwerte sowohl des vertikalen als auch des horizontalen Geschwindigkeitsfeldes gebildet worden. Die Zeitreihe dieser höhenabhängigen horizontal gemittelten Geschwindigkeitskomponenten wurde einer Poweranalyse unterzogen. Die Abbildungen 6.11 und 6.12 zeigen für die Modelle tube und pore die Powerspektren der vertikalen Geschwindigkeitskomponente für das schwachmagnetische (oben) bzw. magnetische Teilgebiet (unten) in Einheiten des maximalen Powerwertes des Powerspektrums für das schwachmagnetische Gebiet.
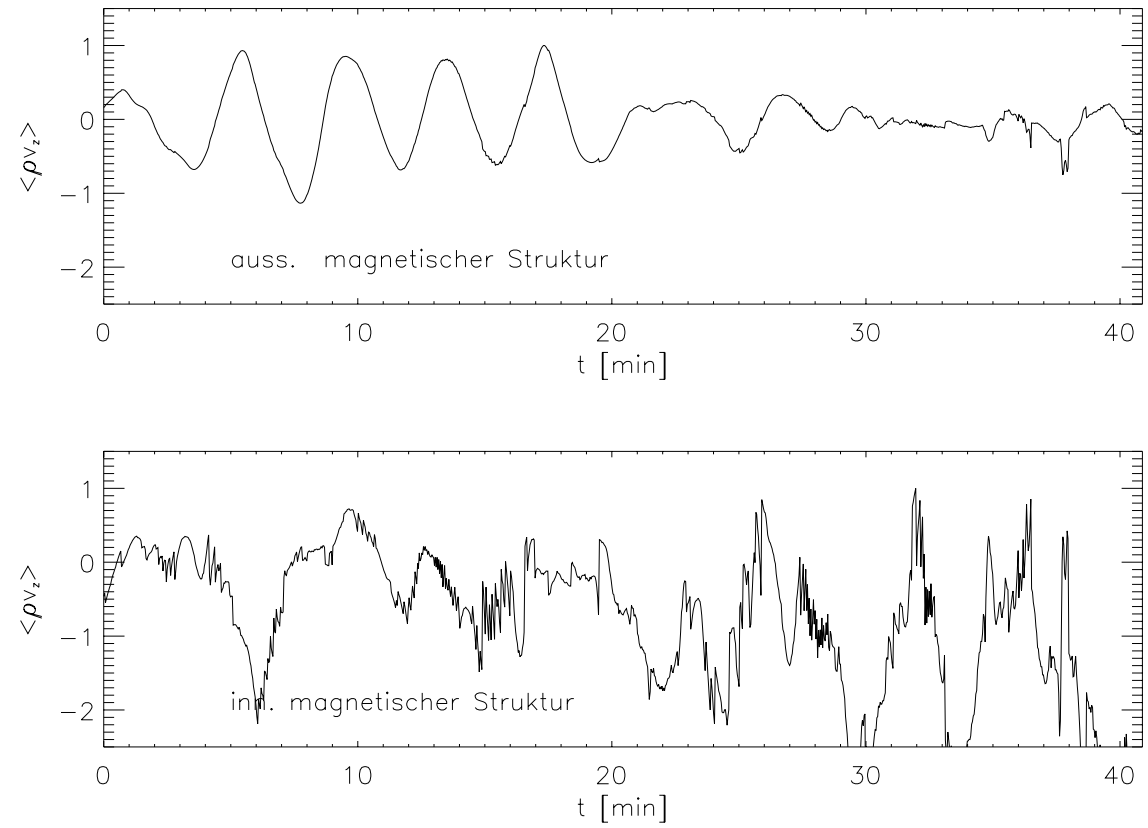

Abbildung 6.9: Zeitliche Entwicklung des horizontal und vertikal gemittelten vertikaler Massenfluß des schwachmagnetischen (oben) und des magnetischen Teilgebietes (unten), beim Modell tube. 


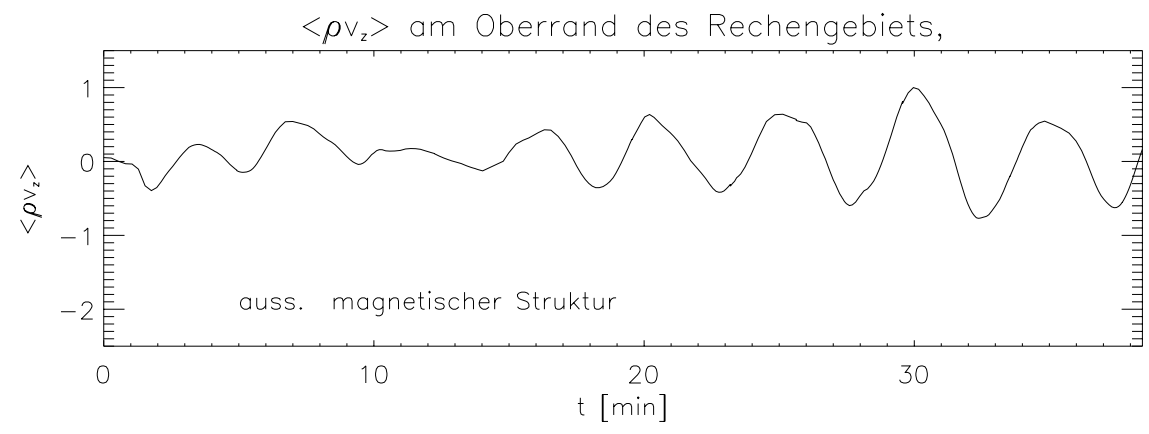

$\left.<\rho \vee_{2}\right\rangle$ am Oberrand des Rechengebiets,

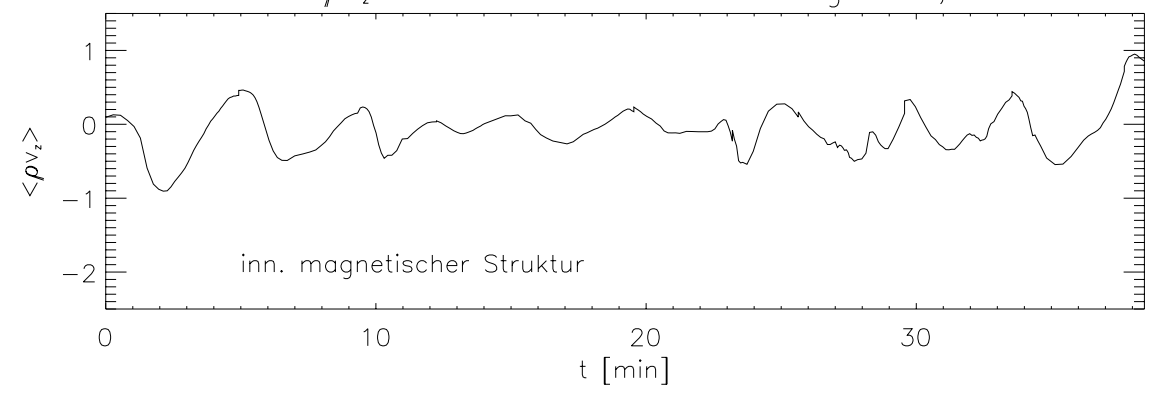

Abbildung 6.10: Zeitliche Entwicklung des horizontal und vertikal gemittelten vertikalen Massenfluß des schwachmagnetischen (oben) und des magnetischen Teilgebietes (unten), beim Modell pore. 

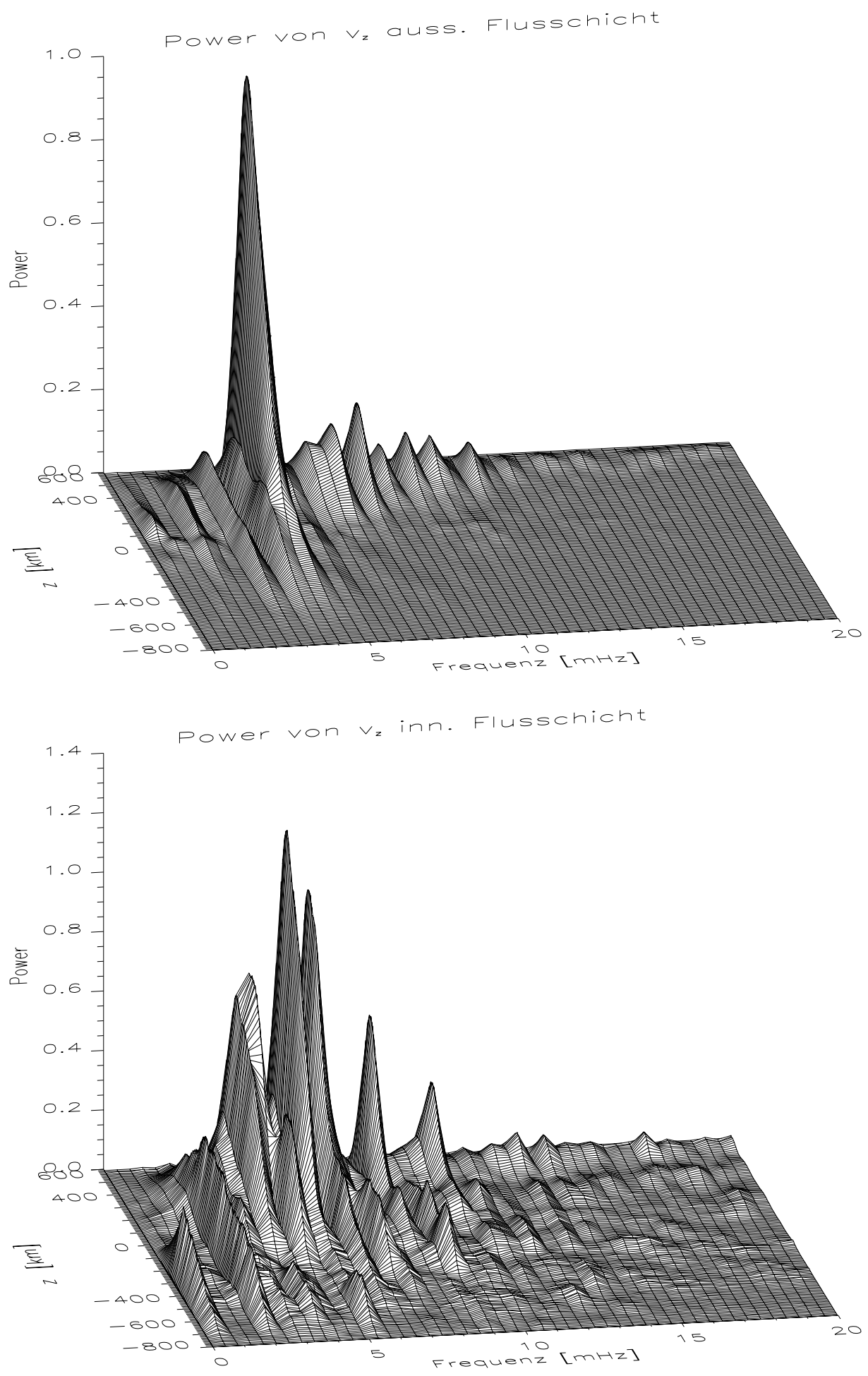

Abbildung 6.11: Oben: Powerspektrum des vertikalen Geschwindigkeitsfeld $v_{z}$ des schwachmagnetischen Teilgebietes des Modells tube, als Funktion der geometrischen Tiefe $z$ im Modell und der Frequenz. Unten: Entsprechend das für das magnetische Teilgebiet. Beide Powerspektren sind in Einheiten des maximalen Powerwertes des Powerspektrums für das schwachmagnetische Gebiet dargestellt. 

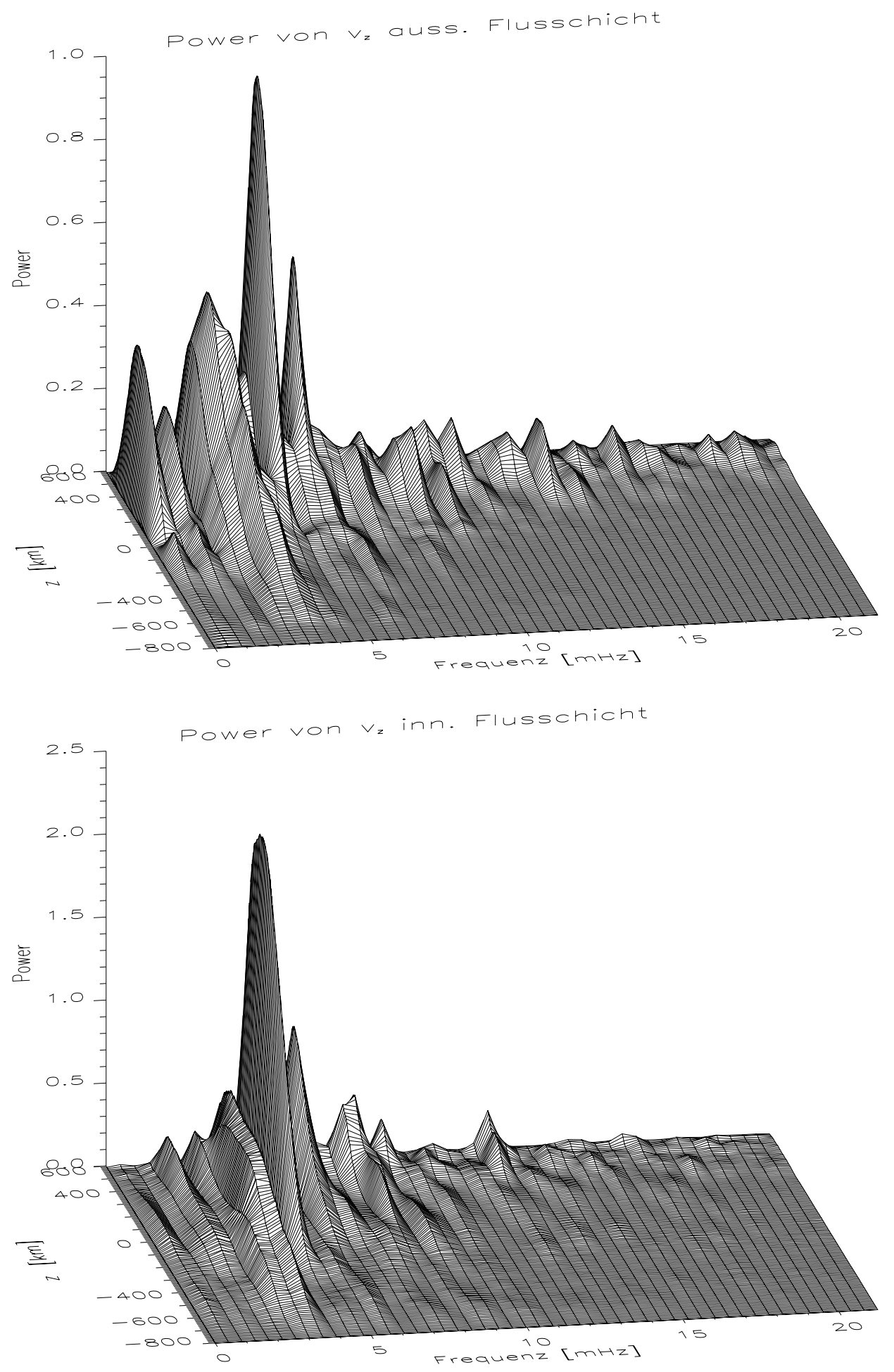

Abbildung 6.12: Oben: Powerspektrum für das Modell pore des vertikalen Geschwindigkeitsfeld $v_{z}$ des schwachmagnetischen Bereichs als Funktion der geometrischen Tiefe $z$ in der Atmosphäre und der Frequenz. Unten: Entsprechend das für das magnetische Teilgebiet. Beide Powerspektren sind in Einheiten des maximalen Powerwertes des Powerspektrums für das schwachmagnetische Gebiet dargestellt. 
Für beide Modelle gibt es im schwachmagnetischen Fall einen dominanten Schwingungsmode bei etwa $4 \mathrm{mHz}$ und kleinere Beiträge bei 1-3, 5-6, 7 und $8 \mathrm{mHz}$ [Abb. 6.11 (oben), 6.12 (oben)]. Im magnetischen Gebiet gibt es neben dem dominanten 4 $\mathrm{mHz}$ Beitrag noch weitere signifikante Beiträge bei 2, 5-6, 8 und $10 \mathrm{mHz}$ [Abb. 6.11 (unten), 6.12 (unten)]. Die Powerspektren für das schwachmagnetische und das magnetische Teilgebiet des Modells pore unterscheiden sich nicht stark voneinander. Dies liegt daran, daß das Verhältnis zwischen magnetischem Fluß und horizontaler Boxausdehnung sehr viel größer ist als beim Modell tube, so daß ein sehr viel größerer Bereich der Rechenbox mit Magnetfeld ausgefüllt ist, da die Magnetfeldkonzentration aufgrund des nach oben hin abnehmenden Gasdrucks in den oberen Schichten sehr stark expandiert. Die Powerbeiträge beim Modell pore sind deutlich größer als die vergleichbaren Beiträge beim Modell tube.

Zur Ausbildung stehender Wellen ist eine aus einer unteren und einer oberen Reflexionsebene aufgebaute Kavität notwendig (Kap. 6.1). Die obere Reflexionsebene, die etwa im Bereich des Temperaturminimums liegt, dort wo die atmosphärischen Cutoff-Frequenz am größten ist, fällt mit dem oberen geschlossenen Rechengebietsrand zusammen. Die Lage der unteren Reflexionsebene wird durch Gl. (6.16) bestimmt und liegt entsprechend der vertikalen Gesamtausdehnung der Rechenbox für globale auf der Sonne beobachtete Eigenmoden mit $l=130, \ldots, 1000$ im Rechengebietsinneren. Das Frequenzspektrum der globalen Schwingungsmuster reicht von 1.3 bis $5.5 \mathrm{mHz}$. Die Schwingungsmode mit der größten Amplitude liegt bei einer Frequenz von $3.3 \mathrm{mHz}$. Die in den Powerspektren der schwachmagnetischen und magnetischen Teilgebiete der Modelle tube und pore markanten Schwingungsbeiträge bei 4 und $8 \mathrm{mHz}$ repräsentieren stehende akustische Wellen. Der Vergleich dieser stehenden Wellen mit den solaren Eigenmoden mit definiertem $l$, deren Umkehrpunkt gerade mit dem unteren Rand der Box zusammenfallen zeigt, daß die Powerbeiträge der stehenden Wellen aufgrund der vertikalen Ausdehnungen des Rechenbietes unrealistisch groß sind und mit den Bedingungen auf der Sonne nicht übereinstimmen. Im schwachmagnetischen Fall des tube- und des pore-Run entsprechen die kleineren Beiträge bei 5-6 mHz der atmosphärischen Cutoff-Frequenz für die obere Photosphäre (Kap. 6.1), die in Abb. 6.13 (a) dargestellt ist. In beiden magnetischen Fällen zeigt ein Vergleich mit den, aus der in Kap. 6.2 skizzierten linearen Analyse folgenden Cutoff-Frequenzen für die sausage-modes oder longitudinalen Flußröhrenwellen mit und ohne Einbeziehung der Strahlungsdämpfung [Abb. 6.13 (b), (c)], daß die bei 5-6 mHz liegenden Peaks der Cutoff-Frequenz der longitudinalen Flußröhrenwellen und der atmosphärischen Cutoff-Frequenz entsprechen. Erstere berechnen sich nach der Gl. (6.23) (ohne Strahlungsdämpfung) und der Gl. (6.24) (mit Strahlungsdämpfung) unter Verwendung der zeitlich und horizontal gemittelten Vertikalprofile von $\gamma(z), H_{p}(z), v_{A}(z), \tau_{R}(z), c_{S}(z)$ und $c_{T}(z)$. Die bei 9-10 mHz liegenden Beträge könnten von longitudinalen Flußröhrenwellen herrühren, deren Frequenzen größer als die entsprechende Cutoff-Frequenz [Gln. (6.23), (6.24)] sind und von Volkmer et al. (1995) ([103]) auch beobachtet werden. Jede weitere Interpretation der Resultate in Richtung der linearen Resultate erscheint allerdings problematisch, da in beiden Simulationen in den Bereichen für $z>-100 \mathrm{~km}$, die Näherung der dünnen Flußröhren nicht mehr gilt, weil sich die Magnetfeldkonzentration aufgrund des abnehmenden Gasdrucks sehr stark aufweitet und sich der Zustand normaler Schallwellen einstellt. Die Abbildungen 6.14 

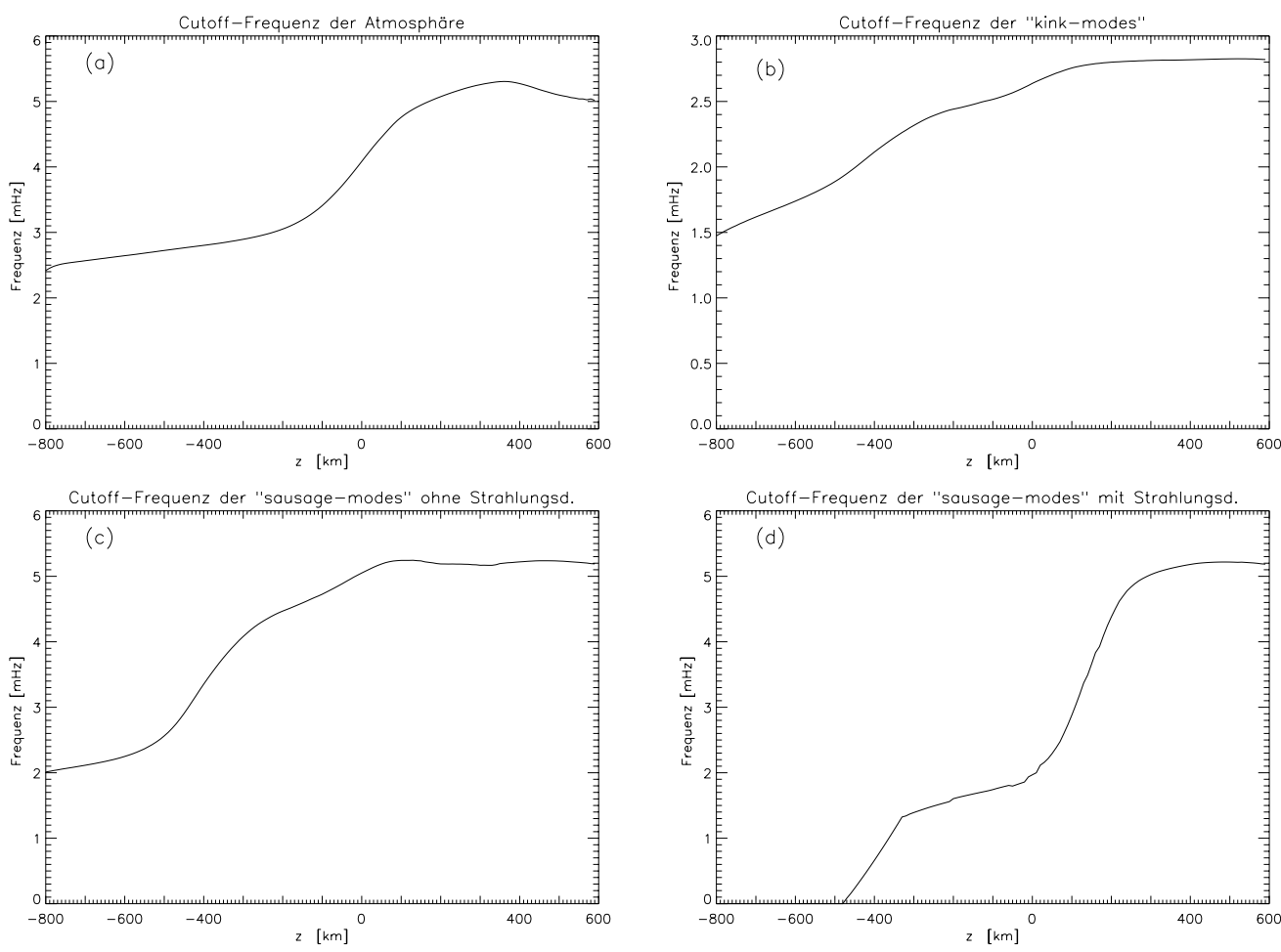

Abbildung 6.13: (a) Lokal Cutoff-Frequenz der Atmosphäre im Falle des tube- und des pore-Modells. (b) Kink-modes oder transversalen Flußröhrenwellen. (c) CutoffFrequenzen innerhalb der magnetischen Struktur für die sausage-modes oder longitudinalen Flußröhrenwellen ohne Strahlungsdämpfung und mit Strahlungsdämpfung (d).

zeigen die Powerspektren der horizontalen Geschwindigkeitskomponente im magnetischen Fall für beide Modelle. Die Beiträge bei $0-2 \mathrm{mHz}$ repräsentieren die hydrodynamische Zeitskala in der granulare Muster vergehen und neuentstehen und verdeutlichen somit den Einfluß der hydrodynamischen Strömungen, die auch das horizontale Schwingungsmuster der Magnetfeldkonzentrationen dominieren. Der Vergleich der Frequenzbereiche, in denen im Powerspektrum deutliche Beiträge vorhanden sind, mit der aus der linearen Analyse folgenden Cutoff-Frequenz der transversalen Flußröhrenwellen oder kink-modes [Abb. 6.13 (b)] zeigt keine Übereinstimmungen. 

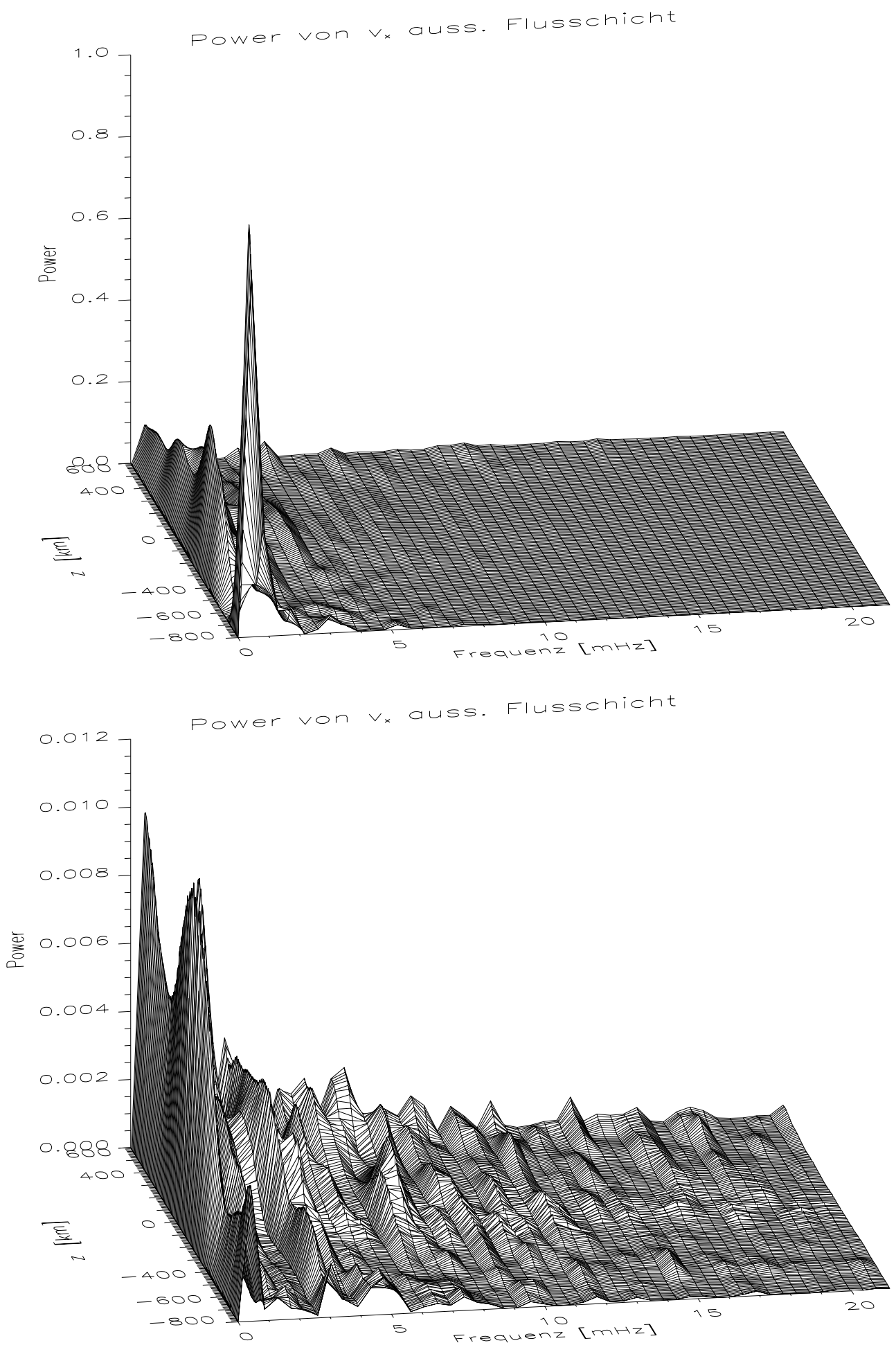

Abbildung 6.14: Powerspektrum des horizontalen Geschwindigkeitsfeld $v_{x}$ des magnetischen Bereichs für die beiden Modelle tube (oben), pore (unten) als Funktion der geometrischen Tiefe $z$ und der Frequenz. Beide Powerspektren sind in Einheiten des maximalen Powerwertes des Powerspektrums für das schwachmagnetische Gebiet dargestellt. 


\subsubsection{Analyse der Resultate des WDV-Code}

Analog zu den mit dem ST-Code durchgeführten Simulationen zeigt die, mit dem WDV-Code durchgeführte 60 Minuten lange Simulation der Wechselwirkung von konvektiven Strömungen und Magnetfeldkonzentrationen, sowohl innerhalb als auch außerhalb von Magnetfeldkonzentrationen Schwingungsmuster. Für drei der sich innerhalb der ersten 15 Minuten gebildeten Magnetfeldkonzentrationen (Kap. 5) sind diese Schwingungsmuster für die folgenden 40 Minuten in Abb. 6.15 dargestellt. Zur Analyse wurde das gesamte Rechengebiet analog zum Fall der STSimulationen in ein schwachmagnetisches und magnetisches Teilgebiet unterteilt. Die Analyse des magnetischen Teilgebiets zerfiel dabei in die separate Untersuchung der drei ausgebildeten Magnetfeldkonzentrationen, die in Abbildung 5.1 (ganz unten) mit I, II und III gekennzeichnet sind. Es sind drei horizontale Bereichen, die von $-6000 \mathrm{~km}$ bis $-3500 \mathrm{~km}$, von $-2500 \mathrm{~km}$ bis $-1000 \mathrm{~km}$ und von $500 \mathrm{~km}$ bis 3000 $\mathrm{km}$ reichen, definiert worden. In jedem dieser definierten Bereiche wurde als höhenund zeitunabhängiger Indikator zur Unterscheidung zwischen magnetischem und schwachmagnetischem Teilbereich die Größe der Feldstärke $B_{z}$ herangezogen. Als magnetisch ist in jeder Höhe $z$ der Teilbereich bezeichnet worden in dem $B_{z}>500$ Gauß ist. Als schwachmagnetisch derjenige in dem $B_{z}<500$ Gauß war. Zu jedem Zeitpunkt ist anschließend das vertikale Geschwindigkeitsfeld für jeden der drei Bereiche sowohl für das magnetische als auch für das schwachmagnetische Gebiet horizontal gemittelt worden. Im Anschluß daran sind die Zeitreihen einer Poweranalyse unterzogen worden. Die Abbildung 6.16 zeigt das Powerspektrum des vertikalen Geschwindigkeitsfeld des schwachmagnetischen Gebiets (oben) und das Powerspektrum des vertikalen Geschwindigkeitsfeld in der Magnetfeldkonzentration I (unten). Im Vergleich zum letzteren sind in der Abbildung 6.17 die vergleichbaren Powerspektren der Magnetfeldkonzentrationen II (oben) und III (unten) dargestellt. Alle Powerspektrum sind mittels der Bildung eines laufenden Mittelwertes über jeweils 3 Punkte geglättet und die Powerbeiträge in Einheiten des maximalen Powerbeitrags des Powerspektrums für das schwachmagnetische Gebiet dargestellt worden. Das Powerspektrum für das schwachmagnetische Gebiet zeigt deutliche Powerbeiträge bei 0.5-2, 3-5 und 6-7 mHz. Während die markanten Beiträge im Frequenzbereich von $0.5-2 \mathrm{mHz}$ die hydrodynamische Zeitskala in der granulare Muster vergehen und neuentstehen repräsentiert. Sie verdeutlichen somit den Einfluß der hydrodynamischen Strömungen. Die Beiträge bei 3-5 und 6-7 mHz haben stehende akustische Wellen deren Entstehung in Kap. 6.3.1 bereits diskutiert wurde und die atmosphärische Cutoff-Frequenz, die in Abbildung 6.18 (a) dargestellt ist als Ursache. 

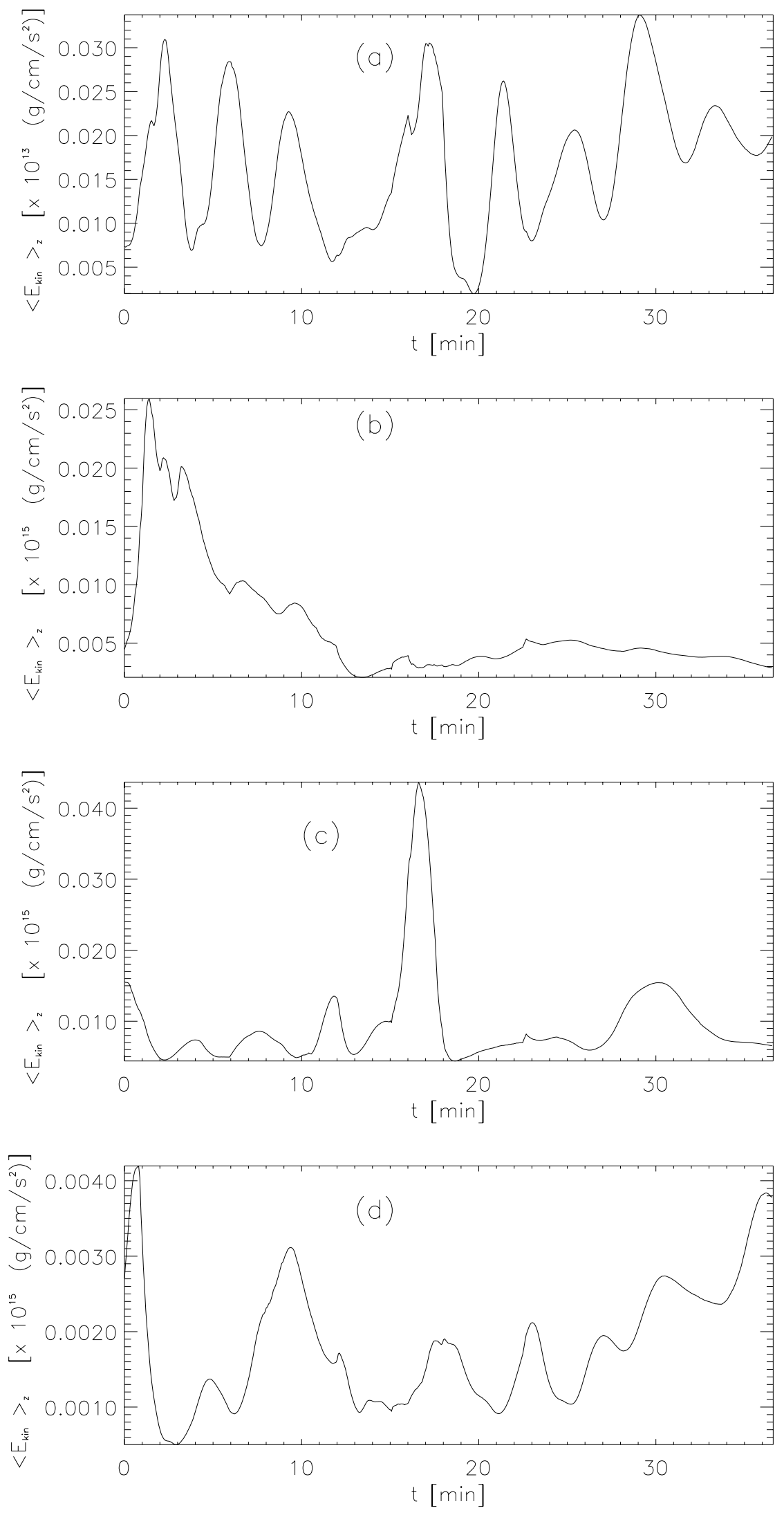

Abbildung 6.15: Horizontal und vertikal gemittelte kinetische Energie des schwachmagnetischen (a) und für die drei untersuchten Magnetfeldkonzentrationen I (b), II (c), III (d). 

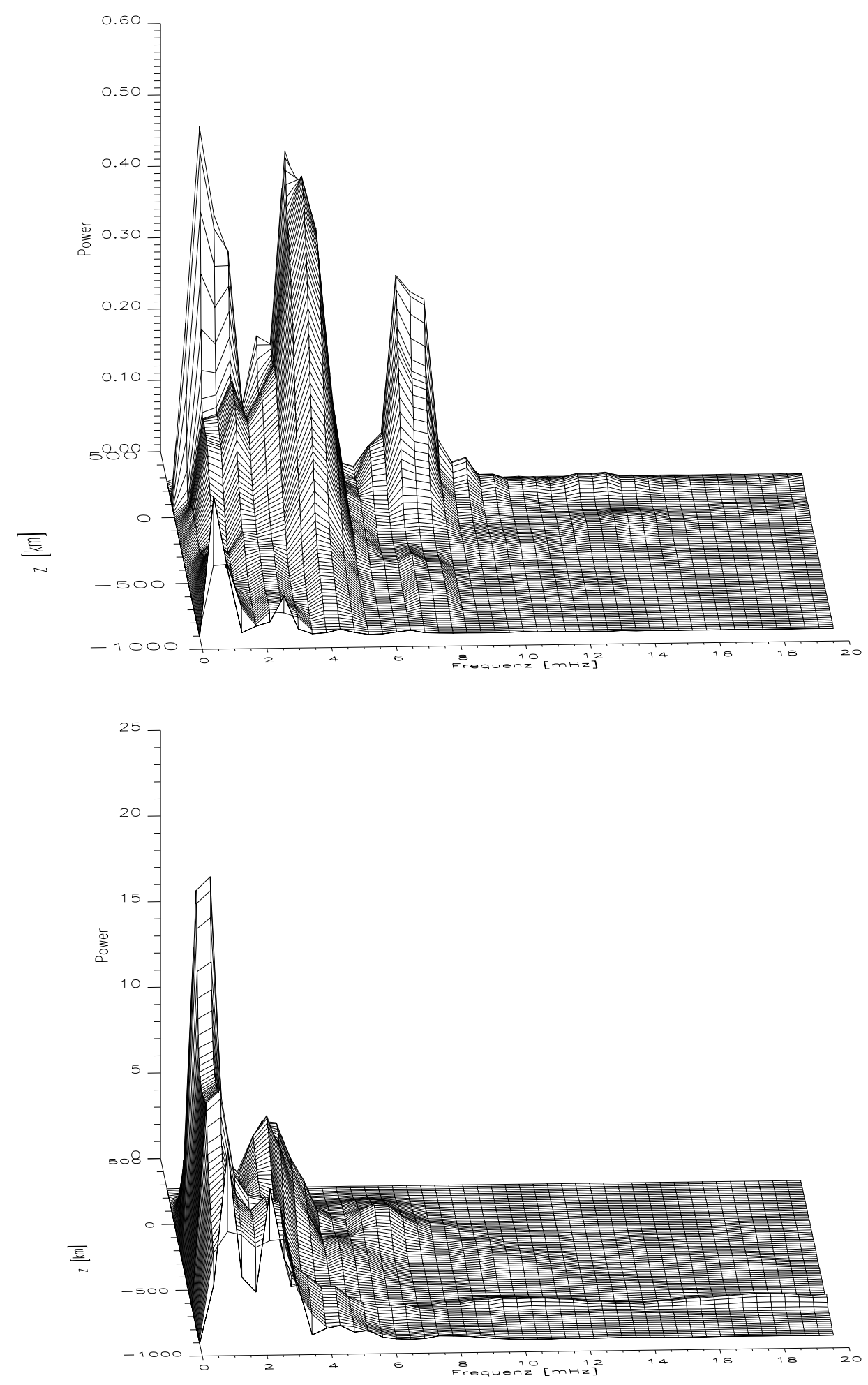

\begin{abstract}
Abbildung 6.16: Oben: Powerspektrum des vertikalen Geschwindigkeitsfeld $v_{z}$ im schwachmagnetischen Bereich als Funktion der geometrischen Tiefe $z$ und der Frequenz. Unten: Powerspektrum des vertikalen Geschwindigkeitsfeld $v_{z}$ innerhalb der Magnetfeldkonzentration I. Die Powerbeiträge sind in Einheiten des maximalen Powerbeitrags des Powerspektrums für das schwachmagnetische Gebiet dargestellt.
\end{abstract}



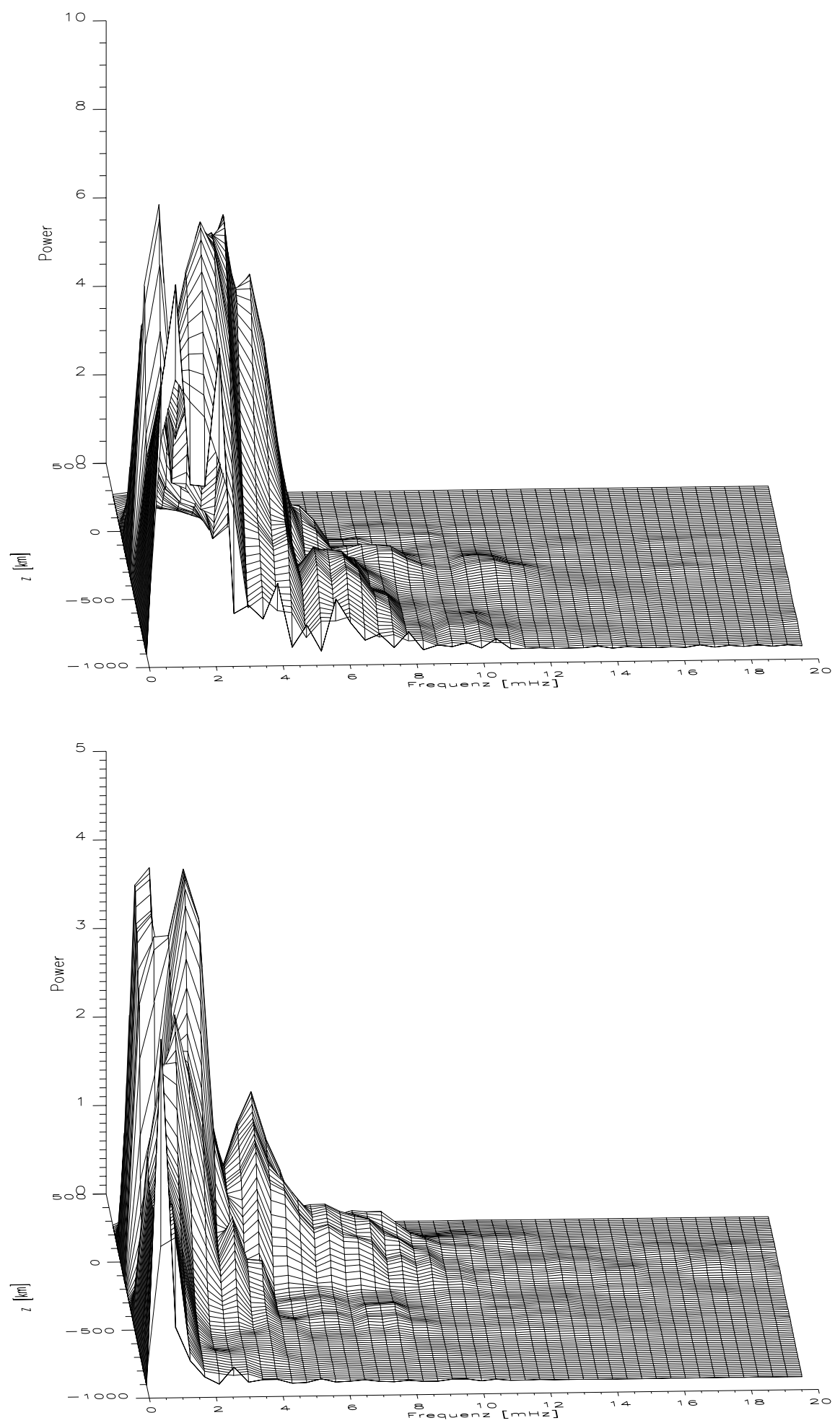

Abbildung 6.17: Oben: Powerspektrum des vertikalen Geschwindigkeitsfeld vz innerhalb der Magnetfeldkonzentration II, als Funktion der geometrischen Tiefe $z$ und der Frequenz. Unten: Analog für die Magnetfeldkonzentration III. Die Powerbeiträge sind in Einheiten des maximalen Powerbeitrags des Powerspektrums für das schwachmagnetische Gebiet dargestellt. 
Die Powerspektren der drei betrachteten Magnetfeldkonzentrationen I, II, und III sind in den Abbildungen 6.16 (unten) und 6.17 [(oben), (unten)] gezeigt. Das der Magnetfeldkonzentration I zugehörende Powerspektrum zeigt deutliche Beiträge bei 0-2 und bei 3-4.5 mHz. Erstere rühren von dem in Abbildung 6.15 (b) gezeigten innerhalb der ersten 12 Minuten bestehenden Trend im Geschwindigkeitsfeld her. Dieser könnte zum Teil in den, die Magnetfeldkonzentration umgebenden hydrodynamischen Strömungen und deren typischen Zeitskala von 10-12 Minuten die Ursache haben. Die Beiträge bei $3-4.5 \mathrm{mHz}$ wurden von stehenden akustischen Wellen bewirkt. Es gibt keine nennenswerten Beiträge für Frequenzen $\nu>5 \mathrm{mHz}$. Das Powerspektrum der Magnetfeldkonzentration II zeigt im Bereich von 0-4 mHz markante Beiträge, die die gleiche Ursache wie im Fall I haben könnten. Es zeigt keine Beiträge bei der Cutoff-Frequenz der longitudinalen Flußröhrenwellen. Das Powerspektrum der Magnetfeldkonzentration III zeigt drei deutliche Beiträge bei 0$4 \mathrm{mHz}$ und 6-8 $\mathrm{mHz}$, die nach zunehmenden Frequenzen geordnet analog zu I und II die Ursache einer langfristigen durch die externen Strömungen bewirkten Entwicklung sein kann und akustischen Eigenschwingungen und der Cutoff-Frequenz der longitudinalen Flußröhrenwellen zugehören. Der Vergleich der innerhalb der Magnetfeldkonzentrationen vorhandenen Powerbeiträge bei $6 \mathrm{mHz}$, die die Cut-off Frequenz der longitudinalen Flußröhrenwellen repräsentieren, mit der in Kap. 6.2 erläuterten Cutoff-Frequenz für die sausage-modes, die in Abb. 6.18 (b), (c) und (d) dargestellt sind, zeigt im Rahmen der Frequenzungenauigkeit, die von der Gesamtlänge der Zeitreihe (40 min) bestimmt wird und bei $0.4 \mathrm{mHz}$ liegt, nur im Fall der Magnetfeldkonzentration III leichte Übereinstimmungen. Diese CutoffFrequenzen wurden ohne Berücksichtigung der Strahlungsdämpfung für die drei Magnetfeldkonzentrationen nach der Gl. (6.23) unter Verwendung der zeitlich und horizontal gemittelten Vertikalprofile von $\gamma(z), H_{p}(z), v_{A}(z), \tau_{R}(z), c_{S}(z)$ und $c_{T}(z)$ für I, II und III berechnet. Der Fall mit Strahlungsdämpfung unterscheidet sich nicht wesentlich und wird daher nicht dargestellt. Allerdings ist wie schon im Falle der ST-Resultate (Kap. 6.3.1) erläutert, jede Interpretation der Powerbeiträge in Richtung der linearen Resultate, aus bereits erläuterten Gründen problematisch. Keine der untersuchten Magnetfeldkonzentrationen zeigt deutliche Schwingungsbeiträge, welche den in Kap. 5 beschriebenen, in den ersten 15 Minuten mit einer Periode von etwa 3 Minuten stattfindenden Magnetfeldverstärkungen eindeutig zuweisbar sind. Dies liegt zum einen daran, daß zur Schwingungsanalyse nur die Resultate schon ausgebildeter Magnetfeldkonzentrationen herangezogen werden (für $t>20 \mathrm{~min}$ ) und diese Schwingungsbeiträge entweder durch die numerische Diffusion bereits weggedämpft sein könnten oder weil aufgrund der starken radiativen Abkühlung innerhalb der Magnetfeldkonzentrationen die Temperatur und der Gasdruck mit der Zeit so stark abnahmen, daß für $t>20 \min \beta \ll 1$ wurde, sich der Zustand reiner Schallwellen mit Ausbreitungsgeschwindigkeiten $\propto \sqrt{T}$ einstellte und die zugehörigen Perioden entsprechend größer wurden und somit keine eindeutige Zuweisung zwischen den Powerbeiträgen und den Periode der Magnetfeldverstärkungen vornehmen lassen. Ein qualitative Untermauerung dieser Vermutung stellt der Vergleich der Schwingungsperioden stehender akustischer Wellen innerhalb der Magnetfeldkonzentrationen dar. Während die Frequenzen innerhalb der Magnetfeldkonzentrationen (I, II, III) nach der 10. Minute 3.3, 3.2 und $3.2 \mathrm{mHz}$ betrugen, nahmen die Ausbreitungsgeschwindigkeiten für $t>20$ min sehr stark ab 
und die Perioden entsprechend zu. Zum Zeitpunkt $t=40$ min betrugen die Frequenzen nur noch 2.1, 1.9 und $2.2 \mathrm{mHz}$. 

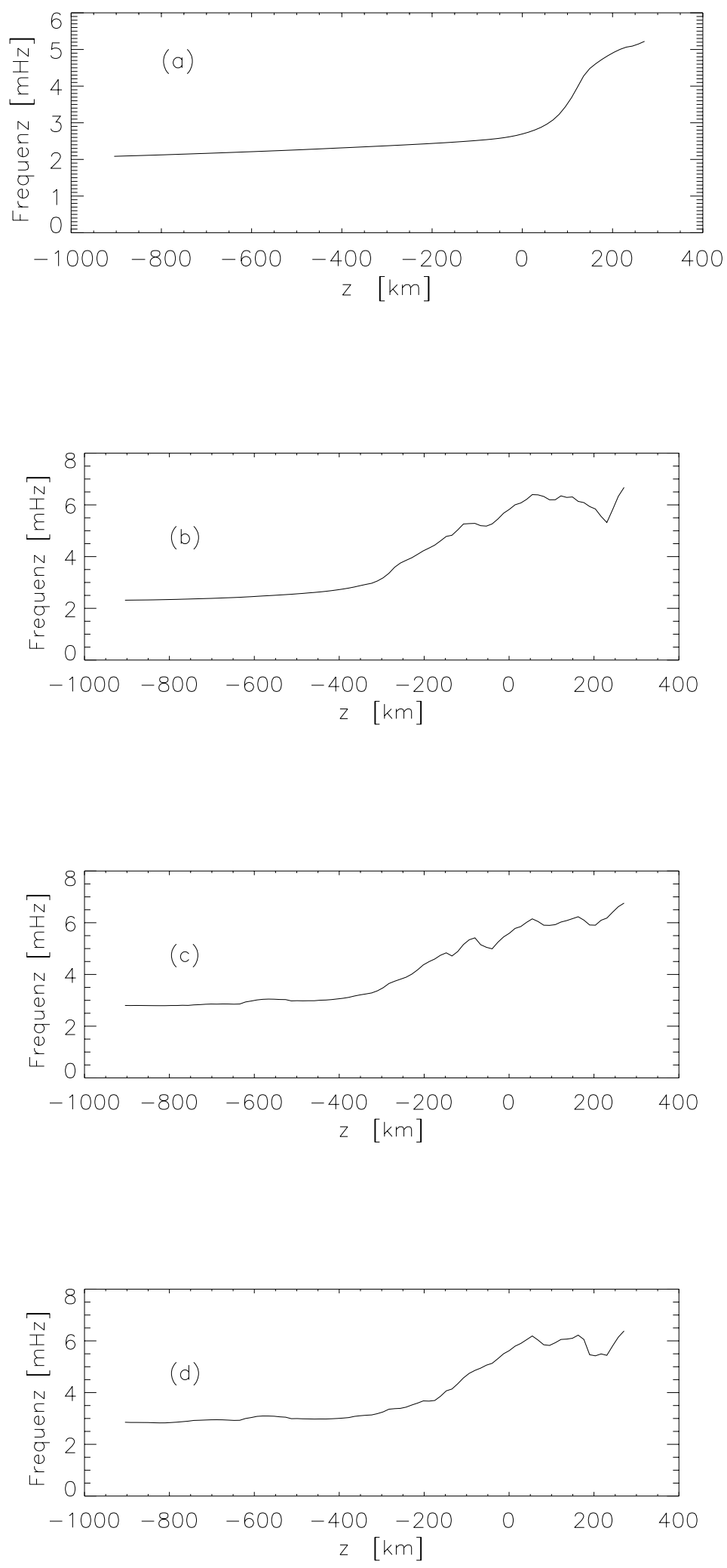

Abbildung 6.18: Lokale Cutoff-Frequenz der mittleren Schichtung (a). CutoffFrequenzen der longitudinalen Flußröhrenwellen ohne Strahlungsdämpfung für die untersuchten Magnetfeldkonzentrationen I (b), II (c) und III (d). 
Die Abbildung 6.19 zeigt das Powerspektren der horizontalen Geschwindigkeitskomponente im Fall der Magnetfeldkonzentration I. Da sich die Powerspektren für die anderen beiden Magnetfeldkonzentrationen nur unwesentlich von dem der Magnetfeldkonzentration I unterscheiden, wird auf sie nicht weiter eingegangen. Der Hauptbeitrag liegt im Bereich von 0-2 $\mathrm{mHz}$ und ist analog zu den Powerspektren der vertikalen Geschwindigkeitskomponente die Ursache eines langfristigen Trends, der von den außerhalb der Magnetfeldkonzentrationen existierenden hydrodynamischen Strömungen bewirkt wurde.

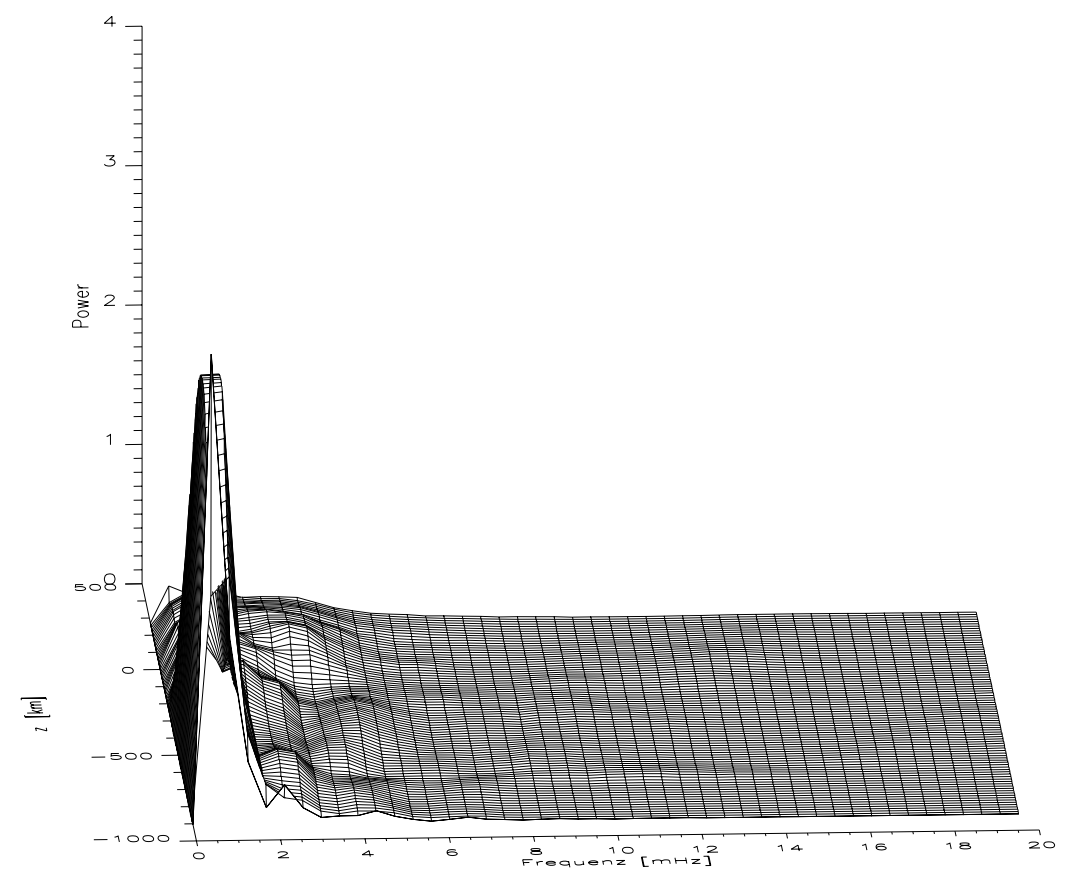

Abbildung 6.19: Powerspektrum des horizontalen Geschwindigkeitsfeld v im Fall der Magnetfeldkonzentration I. Die Powerbeiträge sind in Einheiten des maximalen Powerbeitrags des Powerspektrums für das schwachmagnetische Gebiet dargestellt.

\subsubsection{Diskussion}

Es wurden drei Simulationsläufe analysiert. Zwei davon wurden mit dem ST-Code und einer mit dem WDV-Code durchgeführt. Die unterschiedlichen Konfigurationen sind in Tab. 6.1 erläutert. Da der WDV-Code mit einem wesentlich besseren Strahlungstransportlöser arbeitet, 5 mal grössere horizontale Ausdehnung besitzt und mit einem offenen unteren Rand arbeitet, sollten die mit ihm berechneten Lösungen den wirklichen Verhältnissen in den untersuchten solaren Bereichen näher kommen, als die mit dem ST-Code erzielten Resultate. Im schwachmagnetischen Bereich gibt es bei den ST-Resultaten nur bei $4 \mathrm{mHz}$ von stehenden akustischen Wellen bewirkte deutliche Powerbeiträge. Kaum nennenswerte Powerbeiträge gibt es im Frequenzbereich von 5-10 mHz. Die WDV-Resultate für den 
schwachmagnetischen Fall hingegen zeigen zusätzlich zu dem $4 \mathrm{mHz}$ Beitrag vergleichbar große Beiträge im Bereich von 0-2 und 6-8 mHz, die der Konvektion und der atmosphärischen Cut-off Frequenz entsprechen. Im magnetischen Fall zeigen die mit dem ST-Code gewonnenen Resultate sowohl im Modellfall tube, als auch im Modellfall pore signifikante Beiträge bei 3, 4, 6, 8, und $10 \mathrm{mHz}$. Dabei sind die Beiträge bei 3-4 mHz am größten. Die Beiträge bei $6-8 \mathrm{mHz}$ entsprechen der Cut-off der longitudinalen Flußröhrenwellen. Beiträge bei $10 \mathrm{mHz}$ rühren von sich ausbreitenden akustischen Wellen her. Die WDV-Resultate im magnetischen Fall zeigen für alle drei Magnetfeldkonzentrationen signifikante Beiträge zwischen 0 und $3.5 \mathrm{mHz}$. Das Maximum im Fall I liegt im Bereich von $0.5-2 \mathrm{mHz}$ und ist die Ursache eines langfristigen Trends in der vertikalen Geschwindigkeitskomponente, der zum Teil in den die Magnetfeldkonzentration umgebenden hydrodynamischen Strömungen die Ursache haben könnte. Kleinere Beiträge bei 3-4 mHz werden von akustischen Eigenschwingungen bewirkt. Im Fall II liegt das Maximum wiederum im Bereich von 0.5-2 $\mathrm{mHz}$. Weitere Beiträge, die dieselben Ursachen haben wie im Fall I, liegen bei $3.5 \mathrm{mHz}$. Im Fall III verhält es sich annährend wie im Fall II, mit dem Zusatz, daß der Beitrag bei $3.5 \mathrm{mHz}$ sehr viel breiter ist und bis 4.5 $\mathrm{mHz}$ reicht. Die Beiträge bei 0.5 und $2 \mathrm{mHz}$ gibt es in den ST-Resultaten nicht. Die im Falle der WDV-Simulationen existierenden markanten Beiträge im Bereich von 3-4 $\mathrm{mHz}$ werden akustischen Eigenschwingungen zugeschrieben und lassen sich aufgrund des Frequenzbereichs mit globalen Eigenschwingungen mit definiertem $l$ $(l=130, \ldots, 1000)(5-m i n$ Oszillationen), deren untere und obere Reflexionsebene gerade mit dem Rechengebietsunter- und Rechengebietsoberrand zusammenfallen, vergleichen. Diese Beiträge gibt es im ST-Falle auch, allerdings gibt es im WDV-Fall keine Beiträge im Bereich von 6-10 mHz. Die von Volkmer et al. (1995) ([103]) beobachtet wurden. Sie scheinen durch die in Kapitel 5.2.1 bereits erwähnte numerische Diffusion sehr stark abgedämpft und somit 'ausgeschmiert' zu werden. Es werden nur die großskaligen wahrgenommen. 


\section{Kapitel 7}

\section{Ausblick}

Die mittels zweidimensionaler Simulationen gewonnenen Erkenntnisse über die Wechselwirkungen von kleinskaligen Magnetfeldkonzentrationen und konvektiven Strömungen und die damit direkt verbundenen Schwingungsmuster der Magnetfeldkonzentrationen geben einen Einblick in die in diesen atmosphärischen Bereichen ablaufenden physikalischen Prozesse. Allerdings lassen sich die quantitativen Resultate nur unter Vorbehalt auf die Sonne übertragen, da die Strömungsmuster im gesamten Rechengebiet aufgrund der fehlenden dritten Raumdimension von den realen Strömungszuständen abweichen. Somit stellen die durchgeführten zweidimensionalen Simulationen eine Vorstufe auf dem Weg zu dreidimensionalen Simulationen dar und dienen als Test der numerischen Verfahren im Rahmen einer realen Anwendung. Gemeinsam mit A. Dedner, M. Wesenberg entwickle ich gegenwärtig einen 3D-MHD-Code, der bis auf die zusätzliche Raumdimension alle Eigenschaften des WDV-Code besitzt und mit dem in Zukunft realistische dreidimensionale Simulationen der Wechselwirkung von Magnetfeld und konvektiven Strömungen durchgeführt werden sollen. 


\section{Kapitel 8}

\section{Anhang}

\subsection{Anhang A: Impliziter-Runge-Kutta-Löser (IRK- Methode)}

Wie schon in Kap. 4.2.1 kurz erwähnt gab es bei der numerischen Lösung der Strahlungstransportgleichung Gl. (4.1) Probleme beim Einsatz expliziter Verfahren (explizite RK, Gauss-Legendre, Romberg etc.) Dies ist in der Tatsache, daß der Absorptionskoeffizient $\kappa$ für atmosphärische Bereiche $\tau \geq \tau_{5000}=1$ proportional $T^{10}$ und $\rho^{3}$ ist, begründet. Aufgrund dieser Abhängigkeiten spricht man auch von einem 'steifen System'. Steife Systeme sind im allgemeinen dadurch charakterisiert, daß sich die Lösung am Anfang des Integrationsintervalls sehr schnell ändert (man spricht von der transienten Phase) und dann sehr bald die glatte Phase erreicht (Abb.8.1). In dieser sind die Ableitungen der Lösung $y(t)$ von moderater Größe $\left(\left\|y^{(l)}(t)\right\|=\mathcal{O}(1)\right)$. Somit empfahl sich zur Lösung der linearen gewöhn-

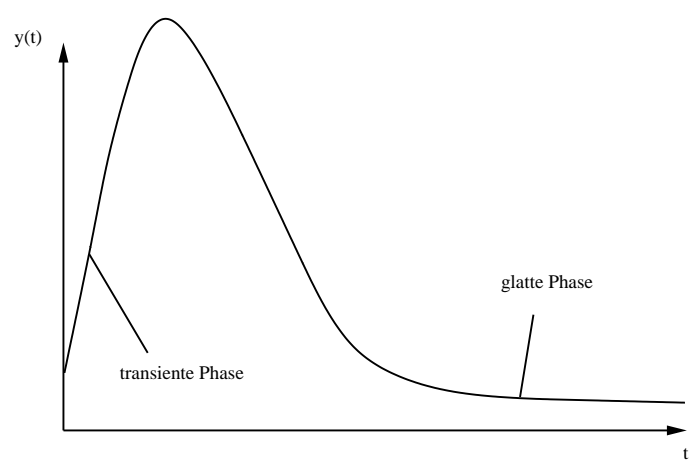

Abbildung 8.1: Transiente und glatte Phase einer steifen Lösung

lichen DGL. (4.14) der Einsatz eines impliziten Runge-Kutta-Verfahrens. Dieses erfordert im Falle einer linearen DGL. nur die Lösung eines linearen Gleichungssystems. Formal stellt sich die Klasse der impliziten Runge-Kutta-Verfahren für ein 
spezielles Anfangswertproblem

$$
y^{\prime}(t)=f(t, y(t)), \quad y\left(t_{0}\right)=y_{0}
$$

und sein Integraläquivalent

$$
y(t)=y_{0}+\int_{t 0}^{t} f(\tau, y(\tau)) d \tau
$$

folgendermaßen dar ([95]):

$$
\begin{aligned}
c_{i} & =\sum_{j=1}^{n} \beta_{i j} \\
k_{i} & =f\left(x_{n}+c_{i} h, y_{n}+h \sum_{j=1}^{n} \beta_{i j} k_{i}\right) \\
\vec{\gamma} & =\left(\gamma_{1}, \gamma_{2}, \ldots\right)^{T}, \text { (Wichtungsvektor) } \\
y_{n+1} & =y_{n}+h \sum_{i=1}^{n} \gamma_{i} k_{i}
\end{aligned}
$$

Konkret läßt sich nun die Strahlungstransportgleichung Gl.(4.1) entlang einer 'shortcharakteristic' mittels eines impliziten Runge-Kutta-Verfahrens in nachfolgend dargestellter Form lösen.

$$
\begin{aligned}
& I_{h}=I_{0}+\frac{1}{\vec{\mu}} \int_{0}^{h} f(\tau, I(\tau)) d \tau=\int_{0}^{h}(\kappa(\tau) \rho(\tau) S(\tau)-\kappa(\tau) \rho(\tau) I(\tau)) d \tau \\
& I_{h}=I_{0}+h\left(\gamma_{1} k_{1}+\gamma_{2} k_{2}\right) \\
& k_{1}=f\left(t_{n}+c_{1} h, y_{n}+h\left(\beta_{11} k_{1}+\beta_{12} k_{2}\right)\right) \\
& k_{2}=f\left(t_{n}+c_{2} h, y_{n}+h\left(\beta_{21} k_{1}+\beta_{22} k_{2}\right)\right)
\end{aligned}
$$

Mit $f(\tau, I(\tau))=\kappa \rho S-\kappa \rho I$ folgt:

$$
\begin{aligned}
& k_{1}=\kappa_{1} \rho_{1} S_{1}-\kappa_{1} \rho_{1} I_{0}-\kappa_{1} \rho_{1} \beta_{11} k_{1} h-\kappa_{1} \rho_{1} \beta_{12} k_{2} h \\
& k_{2}=\kappa_{2} \rho_{2} S_{2}-\kappa_{2} \rho_{2} I_{0}-\kappa_{2} \rho_{2} \beta_{21} k_{1} h-\kappa_{2} \rho_{2} \beta_{22} k_{2} h
\end{aligned}
$$

für $k_{1}, k_{2}$ ein Gleichungssystem mit zwei Unbekannten:

$$
\left(\begin{array}{cc}
1+\kappa_{1} \rho_{1} \beta_{11} h & \kappa_{1} \rho_{1} \beta_{12} h \\
\kappa_{2} \rho_{2} \beta_{12} & 1+\kappa_{2} \rho_{2} \beta_{22} h
\end{array}\right)\left(\begin{array}{c}
k_{1} \\
k_{2}
\end{array}\right)=\left(\begin{array}{c}
\kappa_{1} \rho_{1}\left(S_{1}-I_{0}\right) \\
\kappa_{2} \rho_{2}\left(S_{2}-I_{0}\right)
\end{array}\right)
$$




$$
\begin{array}{r}
\vec{A}_{i k} \vec{k}=\vec{B} \\
\vec{k}=A_{j k}^{-1} \cdot \vec{B}
\end{array}
$$

Mit $A^{-1}=\frac{\left(A_{j k}\right)^{T}}{\operatorname{det} A}$

$$
\begin{aligned}
& A_{j k}=\left(\begin{array}{cc}
1+\kappa_{1} \rho_{1} \beta_{11} h & \kappa_{1} \rho_{1} \beta_{12} h \\
\kappa_{2} \rho_{2} \beta_{12} & 1+\kappa_{2} \rho_{2} \beta_{22} h
\end{array}\right) \\
& \left(A_{j k}\right)^{T}=\left(\begin{array}{cc}
1+\kappa_{2} \rho_{2} \beta_{22} h & \kappa_{1} \rho_{1} \beta_{12} h \\
-\kappa_{2} \rho_{2} \beta_{21} & 1+\kappa_{1} \rho_{1} \beta_{11} h
\end{array}\right)
\end{aligned}
$$

und

$$
\operatorname{det} A=\left|A_{j k}\right|=\left(1+\kappa_{1} \rho_{1} \beta_{11} h\right)\left(1+\kappa_{2} \rho_{2} \beta_{22} h\right)-\left(\kappa_{1} \rho_{1} \beta_{12} h\right)\left(\kappa_{2} \rho_{2} \beta_{21}\right)
$$

folgt für den Vektor $\vec{k}$ :

$$
\vec{k}=A^{-1} \vec{B}=\frac{1}{\operatorname{det} A}\left(\begin{array}{cc}
1+\kappa_{2} \rho_{2} \beta_{22} h & \kappa_{1} \rho_{1} \beta_{12} h \\
-\kappa_{2} \rho_{2} \beta_{21} & 1+\kappa_{1} \rho_{1} \beta_{11} h
\end{array}\right)\left(\begin{array}{c}
\kappa_{1} \rho_{1}\left(S_{1}-I_{0}\right) \\
\kappa_{2} \rho_{2}\left(S_{2}-I_{0}\right)
\end{array}\right)
$$




\section{Literaturverzeichnis}

[1] J. M. Beckers and E. Schröter, The intensity, velocity and magnetic structure of a sunspot region. I: Observational technique; properties of magnetic knots, Solar Physics 4 (1968), 165.

[2] F. Bendicho, F. Kneer, and J. Trujillo Bueno, On the photospheric temperature in small-scale magnetic flux concentrations, Astron. Astrophys. 264 (1992), 229.

[3] R. B. Blackman and J. W. Tukey, The measurement of power spectra, Dover Publications, 1959.

[4] J.H.M.J. Bruls, P. Vollmöller, and M. Schüssler, Computing radiative heating on unstructured spatial grids, Astron. Astrophys. 348 (1999), 233.

[5] B. G. Carlson, The numerical theory of the neutron transport, Methods in Computational Physics (B. Alder, S. Fernbach, and M. Rotenberg, eds.), Academic Press, London, 1963, 1963.

[6] W. J. Chaplin, Y. Elsworth, G. R. Isaak, C. P. McLeod, B. A. Miller, and $\mathrm{R}$. New, Recent results from the birmingham solar-oscillations network (bison), American Astronomical Society Meeting, American Astronomical Society, 1996.

[7] A. Claverie, G.R. Isaak, C.P. McLeod, H.B. Van der Raay, and T. Roca Cortes, Nature 282 (1979), 591.

[8] J. P. Cox, Theory of Stellar pulsation, Princeton University Press, Princeton, 1980.

[9] L. E. Cram and P. R. Wilson, Hydromagnetic waves in structured magnetic fields, Solar Physics 41 (1975), 313.

[10] W. Dai and P.R. Woodward, A simple riemann solver and higher-order godunov schemes for hyperbolic system of conservation laws, J. Comp. Phys. 121 (1995), 51.

[11] S. F. Davis, Simplified second-order godunov-type methods, SIAM Rev. 9 (1988), 445. 
[12] A. Dedner, Numerik und Analysis für ein gekoppeltes System der EulerGleichungen mit der Strahlungstransportgleichung in zwei Raumdimensionen, Diplomarbeit, University of Freiburg, 1998.

[13] A. Dedner, C. Rohde, and M. Wesenberg, A MHD-simulation in solar physics, Finite Volumes for Complex Applications II: Problems and Perspectives (Paris) (R. Vilsmeier, F. Benkhaldoun, and D. Hänel, eds.), Hermès Science Publications, 1999, pp. 491-498.

[14] A. Dedner and P. Vollmöller, An adaptive higher order method for solving the radiation transport equation on unstructured grids, submitted to J. Comp. Phys. (2001), 1.

[15] A. Dedner and M. Wesenberg, Numerical methods for the reals gas mhd equations, Proceedings of the Eighth International Conference on Hyperbolic Problems: Theory, Numerics, Applications (G. Warnecke, ed.), , 2000, pp. Xy.

[16] R. J. Defouw, Wave propagation along a magnetic tube, Astrophys. J. 209 (1976), 266.

[17] W. Deinzer, G. Hensler, M. Schüssler, and E. Weisshaar, Model calculations of magnetic flux tubes. II. Stationary results for solar magnetic elements, Astron. Astrophys. 139 (1984), 435.

[18] F. L. Deubner, Observations of low wavenumber nonradial eigenmodes of the sun, Astron. Astrophys. 44 (1975), 371.

[19] _ Dynamics of the solar atmosphere. IV-evanescent waves of small amolitude, Astron. Astrophys. 236 (1990), 509.

[20] V. Domingo, B. Fleck, and A. I. Poland, Soho: The solar and heliospheric observatory, Space Science Review 72 (1995), 81.

[21] L.J. Durlofsky, S. Osher, and B. Enquist, Triangle based TVD-Schemes for Hyperbolic Conservation Laws, jcomp 98 (1992), 64-73.

[22] T.G. Duvall and H.W. Harvey, Latitude and depth variation of solar rotation, Nature 302 (1983), 24.

[23] T.G. Duvall, H.W. Harvey, K.G. Libbrecht, B.D. Popp, and M.A. Pomerantz, Frequencies of solar p-mode oscillations, Astrophys. J. 324 (1988), 1158.

[24] T.G. Duvall, H.W. Harvey, and M.A. Pomerantz, Observations of solar oscillations of low and intermediate degree, Nature 321 (1986), 500.

[25] P. M. Edwin and B. Roberts, Wave propagation in a magnetically structured atmosphere. III. - the slab in a magnetic environment, Solar Physics 76 (1982), 239.

[26] C.A.J. Fletcher, Computational techniques for fluid dynamics I+II, Springer, 1991. 
[27] E. Fossat, The iris network for full disk helioseismology - present status of the programme, American Astronomical Society Meeting, IRIS, 1991.

[28] N. T. Frink, Upwind Scheme for Solving the Euler Equations on Threedimensional Unstructured Tetrahedral Meshes, AIAA xx (1990), xx.

[29] N. T. Frink, P. Parkh, and S. Pirzadeh, A Fast Upwind Solver for the Euler Equations on Threedimensional Unstructured Tetrahedral Meshes, AIAA 91 (1991), 102.

[30] T. Geßner, Zeitabhängige Adaption für Finite-Volumen-Verfahren höherer Ordnung, Diplomarbeit, University of Freiburg, 1994.

[31] R. G. Giovanelli, W.C. Livingston, and J.W. Harvey, Motions in solar magnetic tubes II. The Oscillations, Solar Physics 59 (1978), 49.

[32] _ Motions in solar magnetic tubes III. Outward wave propagation in sunspot umbras, Solar Physics 58 (1978), 347.

[33] R. G. Giovanelli and C. Slaughter, Motions in solar magnetic tubes I. The downflow, Solar Physics 59 (1978), 49.

[34] S. K. Godunov, A finite difference method for the computation of diskontinuous solutions of the equations of fluid dynamics, Mat. Sb. 47 (1959), 357.

[35] P. Goldreich and P. Kumar, Wave generation by turbulent convection, Astrophys. J. 363 (1990), 694.

[36] U. Grossmann-Doerth, M. Schüssler, and O. Steiner, Convectice intensification of solar surface magnetic elements:results of numerical experiments, Astron. Astrophys. 337 (1998), 928.

[37] A. Harten, P.D. Lax, and B. van Leer, On upstream differencing and godunov-type schemes for hyperbolic conservation laws, SIAM Rev. 25 (1983), 35.

[38] S. S. Hasan, Convective instability in a solar flux tube. II - nonlinear calculations with horizontal radiative heat transport and finite viscosity, Astron. Astrophys. 143 (1985), 39.

[39] G. Herbold, P. Ulmschneider, H. C. Spruit, and R. Rosner, Propagation of nonlinear, radiatively damped longitudinal waves along magnetic flux tubes in the solar atmosphere, Astron. Astrophys. 145 (1986), 157.

[40] J. Honerkamp and H. Römer, Grundlagen der Klassischen Theoretischen Physik, Springer, 1986.

[41] C. Johnson and J. Pitkäranta, Convergence of a fully discrete scheme for twodimensional neutron transport, SIAM J.Num.Anal. 20 (1983), 951-966.

[42] R. Kippenhahn and C. Möllenhoff, Elementare plasmaphysik, BI Wissenschaftsverlag, 1973. 
[43] F. Kneer, S. S. Hasan, and W. Kalkhofen, Spectral line radiation from solar small-scale flux tubes, Astron. Astrophys. 332 (1998), 1064.

[44] M. Knölker and M. Schüssler, Model calculations of magnetic flux tubes. IV. Convective energy transport and the nature of intermediate size flux concentrations, Astron. Astrophys. 194 (1988), 257.

[45] M. Knölker, M. Schüssler, and E. Weisshaar, Model calculations of magnetic flux tubes. III. Properties of solar magnetic elements, Astron. Astrophys. 194 (1988), 257.

[46] D. Kröner, Numerical schemes for conservation laws, Wiley-Teubner, 1997.

[47] P.B. Kunasz and L. Auer, Short characteristic integration of radiative transfer problems: Formal solution in two-dimensional slabs, J. Quant. Spectrosc. Radiat. Transfer 39 (1988), 67.

[48] R.L. Kurucz, Status of the ATLAS 12 Opacity Sampling Program and New Programs for Rosseland and Distribution Function Opacity, M.A.S.S.; Model Atmospheres and Spectrum Synthesis (S.J. Adelman, F. Kupka, and W.W. Weiss, eds.), ASP Conference Series, 1996, p. 160.

[49] L. Landau and E. M. Lifschitz, Lehrbuch der Theoretischen Physik: Hydrodynamik Band 6, Akademie Verlag, Berlin, 1991.

[50] J. W. Leibacher, J. W. Harvey, F. Hill, R. Hubbard, J. R. Kennedy, J. A. Pintar, A. Bhatnagar, J. A. Kennewell, W. Marquette, J. Patron, O. Saa, and E.;GONG Project Team Yasukawa, The global oscillation network group project, American Astronomical Society Meeting, American Astronomical Society, 1996.

[51] R. B. Leighton, R. W. Noyes, and G. W. Simon, Velocity fields in the solar atmosphere. I. preliminary report, Astrophys. J. 130 (1962), 366.

[52] P. Lesaint and P.A. Raviart, On a finite element method for solving the neutron transport equation, Mathematical Aspects of Finite Elements in Partial Differential Equations (New York) (C. de Boor, ed.), Mathematics Research Center University of Wisconsin-Madison, Academic Press, April 1974, pp. 89-123.

[53] R. LeVeque, Numerical methods for conservation laws, Birkhäuser, Basel; Boston; Berlin, 1990.

[54] M.J. Lighthill, Aerodynamic Phenomena in Stellar Atmospheres (R.N. Thomas, ed.), IAU Symposium, 1952.

[55] H. Lin and T. Rimmele, The granular magnetic fields of the quiet sun, Astrophys. J. 514 (1999), 488.

[56] D. Mihalas, Stellar atmospheres, Freeman and Company, 1978. 
[57] D. Mihalas, L. Auer, and B. Mihalas, Two-dimensional radiative transfer. $i$. planar geometry, Astrophys. J. 220 (1978), 1001.

[58] Z. E. Musielak, R. Rosner, R. F. Stein, and P. Ulmschneider, On sound generation by turbulent convection: A new look at old results, Astrophys. J. 423 (1994), 474.

[59] A Nordlund and R.F. Stein, 3d simulations of solar and stellar convection and magnetoconvection, Comput. Phys. Commun. 59 (1990), 119.

[60] E.N. Parker, Topological dissipation and the small-scale fields in turbulent gases, Astrophys. J. 174 (1972), 499.

[61] _ Hydraulic concentration of magnetic fields in the solar photosphere. IV. Adiabatic cooling and concentration in downdrafts, Astrophys. J. 221 (1978), 368.

[62] _ Magnetic neutral sheets in evolving fields - part two - formation of the solar corona, Astrophys. J. 264 (1983), 635.

[63] _ Magnetic neutral sheets in evolving fields. $i$ - general theory. $i i$ formation of the solar corona, Astrophys. J. 264 (1983), 642.

[64] S. Ploner, Advanced Solar Polarimetry - Theory, Observaton and Instrumentation (M. Sigwarth, ed.), ASP Conference Series, 2001, p. 93.

[65] S. Ploner, S. Solanki, and A. Gadun, The evolution of solar granules deduced from 2-D simulations, Astron. Astrophys. 352 (1999), 679.

[66] W.F. Press, B.P. Flannery, S.A. Teukolsky, and W.T. Vetterling, Numerical recipes, Cambridge Univ Press, Cambridge, 1986.

[67] E. Priest, Solar magnetohydrodynamics, D. Reidel Publishing Company, 1982.

[68] E. R. Priest, Solar magnetohydrodynamics, Reidel Publishing Company, 1982.

[69] M.R.E. Proctor and N. Weiss, Magnetoconvection, Reports on Progress in Physics 45 (1982), 11.

[70] Stein R., Generation of acoustic and gravity waves by turbulence in an isothermal stratified atmosphere, Solar Physics 495 (1967), 385.

[71] B. Roberts, Wave propagation in a magnetically structured atmosphere - II. - waves in a magnetic slab, Solar Physics 69 (1981), 39.

[72] _ Wave propagation in intens flux tubes, Solar Physics 87 (1983), 77.

[73] B. Roberts and A. R. Webb, Vertical motions in an intense magnetic flux tube, Solar Physics 56 (1978), 5. 
[74] _ Vertical motions in an intense magnetic flux tube. III. - on the slender flux tube approximation, Solar Physics 64 (1979), 77.

[75] _ Vertical motions in an intense magnetic flux tube - V. - radiative relaxation in a stratified medium, Solar Physics 68 (1980), 87.

[76] __ Vertical motions in an intense magnetic flux tube. IV. - radiative relaxation in a uniform medium., Solar Physics 68 (1980), 71.

[77] J.R. Rutten and L. Dame, Simuris:high-resolution solar physics, The Magnetic and Velocity Field of Solar Active Regions (H. Zirin, G. Ai, and H. Wang, eds.), ASP Conference Series, 1993, p. 184.

[78] R. Skartlien, Three-dimensional modeling of solar convection and atmosphere dynamics; dissertation, University of Oslo, 1998.

[79] S.K. Solanki, Velocities in solar magnetic fluxtubes, Astron. Astrophys. 168 (1986), 311.

[80] _ Small-scale solar magnetic fields: An overview, Space Science Review 63 (1992), 1.

[81] E. A. Spiegel, The smoothing of temperature fluctuations by radiative transfer, Astrophys. J. 126 (1957), 202.

[82] L. Spitzer, Physics of Fully Ionized Gases, Interscience, New York, 1962.

[83] H. Spruit, Dissertation, University of Utrecht, 1977.

[84] H. C. Spruit, Pressure equilibrium and energy balance of small photospheric fluxtubes, Solar Physics 50 (1976), 269.

[85] _ Convective collapse of flux tubes, Solar Physics 61 (1978), 363.

[86] Motion of magnetic flux tubes in the solar convection zone and chromosphere, Astron. Astrophys. 98 (1981), 155.

[87] _ Propagation Speeds and Acoustic Damping of Waves in Magnetic Flux Tubes, Solar Physics 75 (1982), 3.

[88] M. Steffen, H.-G. Ludwig, and A. Kruess, A numerical simulation study of solar granular convection in cells of different horizontal dimension, Astron. Astrophys. 213 (1989), 371.

[89] R. F. Stein and A. Nordlund, Driving and damping of oscillations, Progress of Seismology of the Sun and Stars (Y. Osaki and H. Shibahashi, eds.), Springer-Verlag, Berlin Heidelberg New York, 1990, 1990, p. 93.

[90] O. Steiner, U. Grossmann-Doerth, M. Knölker, and M. Schüssler, Simulation of the interaction of convective flow with magnetic elements in the solar atmosphere, Astrophys. J. 495 (1998), 468. 
[91] O. Steiner, M. Knölker, and M. Schüssler, Dynamic interaction of convection with magnetic flux sheets: First results of a new MHD-code, Solar Surface Magnetism (R.J. Rutten and C.J. Schrijver, eds.), Kluwer Dordrecht, 1994, p. 441.

[92] M. Stix, The sun, Springer-Verlag, 1991.

[93] F. Stolpe and F. Kneer, High spacial-resolution pectropolarimetry of smallscale solar magnetic fields, Astron. Astrophys. 317 (1997), 942.

[94] _ On weak magnetic flux structures of the Sun, Astron. Astrophys. 353 (2000), 1094.

[95] K. Strehmel and R. Weiner, Numerik gewöhnlicher Differentialgleichungen, Teubner Stuttgart, 1995.

[96] G. Sutmann, Z. E. Musielak, and P. Ulmschneider, Acoustic wave propagation in the solar atmosphere. III. analytic solutions for adiabatic wave excitations, Astron. Astrophys. 340 (1998), 556.

[97] A. Title, T. Tarbell, K. Topka, S. Ferguson, and R. Shine, Statistical Properties Of Solar Granulation Derived From The SOUP Instrument On Spacelab 2, Astrophys. J. 336 (1989), 475.

[98] E.F. Toro, Riemann solvers and numerical methods for fluid dynamics, Springer, 1997.

[99] R.K. Ulrich, The five-minute oscillations on the solar surface, Astrophys. J. 162 (1970), 993.

[100] W. Unno and E. A. Spiegel, The eddington approximation in the radiative heat equation, Publications of the Astronomical Society of Japan 18 (1966), 85 .

[101] P. Venkatakrishnan, Nonlinear development of convective instability within slender flux tubes. I - adiabatic flow, J. Astrophys. Astr. 4 (1983), 135.

[102] _ Nonlinear development of convective instability within slender flux tubes. II - the effect of radiative heat transport, J. Astrophys. Astr. 6 (1985), 21.

[103] R. Volkmer, F. Kneer, and Bendlin C., Short-period waves in small-scale magnetic flux tubes on the sun, Astron. Astrophys. 304 (1995), 1.

[104] N. Weiss, Proc. Roy. Soc. 293 (1982), 310.

[105] M. Wesenberg, A note on mhd-riemann-solvers, J. Comp. Phys. submitted to $(1990), 1$.

[106] _ Finite-Volumenverfahren für die Gleichungen der Magnetohydrodynamik in ein und zwei Raumdimensionen, Diplomarbeit, University of Freiburg, 1998. 
[107] E. Wiehr, Spatial and temporal variations of circular zeeman profiles in isolated solar $\mathrm{Ca}^{+} k$ structures, Astron. Astrophys. 149 (1985), 217.

[108] C. Zwaan, J. J. Brants, and L. E. Cram, High-resolution spectroscopy of active regions. I - Observing procedures, Solar Physics 95 (1985), 3. 


\section{Danksagung}

Mein Dank gilt:

- Meiner Frau Petra und meiner Tochter Antonia.

- Marie, Ev und Hans-Kurt.

- Herrn Prof. M. Schüssler und Herrn Prof. F. Kneer für die Betreuung der Arbeit und die Unterstützung.

- Den Direktoren und Mitarbeitern des Kiepenheuer-Institutes für Sonnenphysik in Freiburg und des Max-Planck-Institutes für Aeronomie in KatlenburgLindau für die Unterstützung bei der Anfertigung dieser Arbeit.

- Herrn A. Dedner und M. Wesenberg für die Bereitstellung des 2-MHDCodes und viele Diskussionen.

- Der Deutschen Forschungsgemeinschaft für die finanzielle Unterstützung dieser Arbeit.

- Dem Rechenzentrum der Universität Freiburg für die Nutzung des Parallelrechners SGI Origin 2000.

- Oski, dem expeditionstauglichen keltischen Allemannen, Cale, Tassos, Jo, dem "Franzosen" Klaus und allen die das Leben im "Wohnklo" erträglicher machten und/oder unter mir zu leiden hatten. 


\section{Lebenslauf}

Geburt: $\quad$ Am 20.03.1966 in Fulda

Eltern: $\quad$ Hans-Kurt Vollmöller

Eveline Vollmöller

Schulbildung: $8 / 72$ bis 7/76 Marquardt-Schule (Grundschule)

$8 / 76$ bis 6/83 Winfried-Schule-Fulda

(Gymnasium)

8/76 bis 6/83 Ferdinand-Braun-Schule

(Gymnasium)

6/86 Abitur

Studium: $\quad$ 10/86 bis 3/88 Physikstudium an der Justus-Liebig-Universität GieBen

6/88 Vordiplomprüfung

2/89 Fortsetzung des Physikstudiums an der Universität Freiburg

3/90 bis 2/92 Diplomarbeit:

„Intensitäts- und Geschwindigkeitsfluktuationen

in der tiefen Photosphäre“

11/92 Diplomprüfung

$11 / 92$ bis 10/93 USA Aufenthalt

10/93 bis 4/95 wissenschaftlicher Angestellter am Fraunhofer-Institut für Atmosphärenphysik, Garmisch-Partenkirchen

4/96 bis 10/01 Doktorarbeit

Während dieser Zeit Tätigkeit als wissenschaftlicher

Mitarbeiter am Kiepenheuer-Institut für Sonnenphysik in Freiburg und am Max-Planck-Institut für Aeronomie in Katlenburg-Lindau 